Aus dem Institut für Tierzucht und Haustiergenetik der Georg-August-Universität Göttingen

\title{
Zuchtplanerische Bewertung verschiedener Strategien für die nachhaltige Zucht ökologischer Milchrinder
}

\author{
Dissertation \\ zur Erlangung des Doktorgrades \\ der Fakultät für Agrarwissenschaften \\ der Georg-August-Universität Göttingen
}

vorgelegt von

Janet Schmidtko

geboren in Hagen 
D 7

1. Referent: Prof. Dr. H. Simianer

2. Korreferentin: PD Dr. R. Baumung

Tag der mündlichen Prüfung: 19. Juli 2007 
Meinen Eltern

Elisabeth und Bernhard Schmidtko 


\section{Inhaltsverzeichnis}

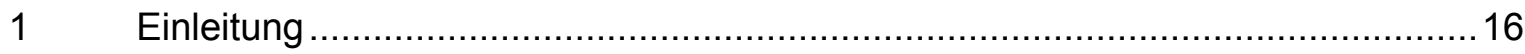

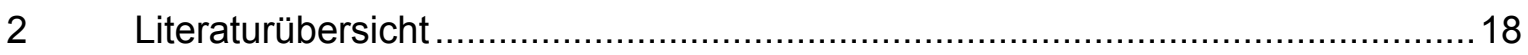

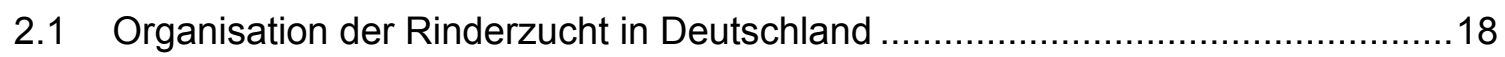

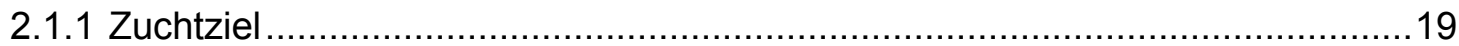

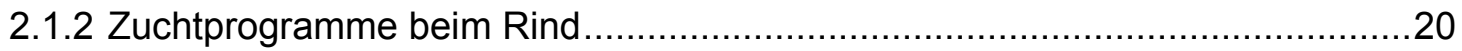

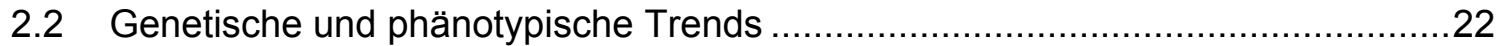

2.3 Die Entstehung des ökologischen Landbaus - ein historischer Rückblick ...........27

2.3.1 Zahlen des ökologischen Landbaus und der Entwicklung der ökologischen Milchviehhaltung in Deutschland............................................29

2.3.2 Richtlinien der ökologischen Milchviehhaltung............................................

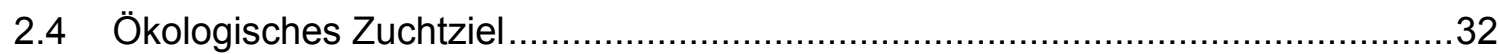

2.4.1 Zuchtorganisationen und Zuchtprogramme der ökologischen

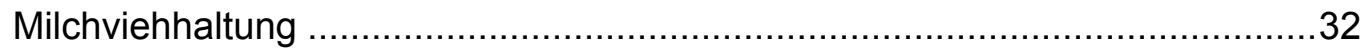

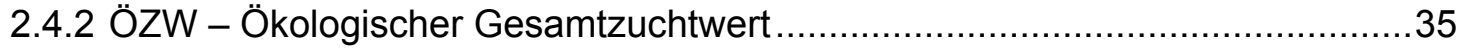

2.5 Genotyp-Umwelt-Interaktionen zwischen konventionellen und ökologisch

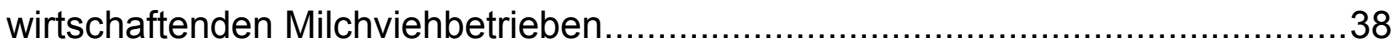

2.6 Zuchtplanung und Optimierung von Zuchtprogrammen ......................................42

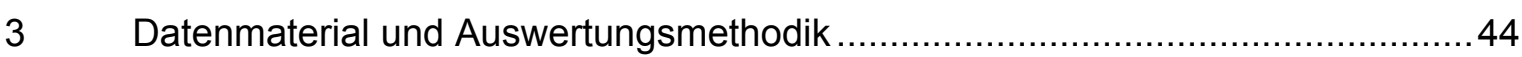

3.1 Datenmaterial ökologischer Milchviehbetriebe in Deutschland ............................44

3.1.1 Genetische und phänotypische Trends der Leistungs- und funktionalen Merkmale bei Bullen und Kühen ökologischer Betriebe

3.1.2 Untersuchung zum ET-Status und Erhebung genetischer Trends ökologischer Bullen im Vergleich zu konventionellen Bullen .46

3.2 ZPLAN - Modellkalkulationen zum Zuchtfortschritt für ein ökologisches

Zuchtprogramm .48

3.2.1 Inputparameter zur Beschreibung der Populationsstruktur des gewählten Zuchtprogramms .49

3.2.1.1 Parameter zur Beschreibung der Ökopopulation ..................................49

3.2.1.2 Biologisch-technische Parameter.....................................................50

3.2.1.3 Parameter zur Beschreibung der Kosten ............................................50

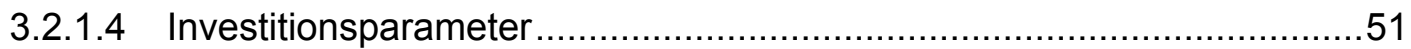

3.2.2 Die Genflussmatrix für das ökologische Zuchtprogramm.............................52

3.2.3 Zuchtwertschätzung mittels Selektionsindex …….......................................54

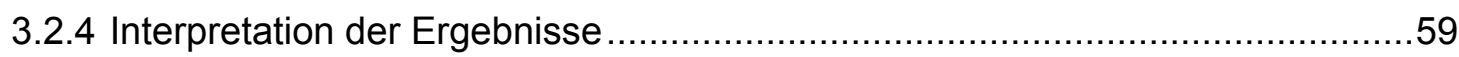


3.2.4.1 Naturaler und monetärer Zuchtfortschritt ..........................................59

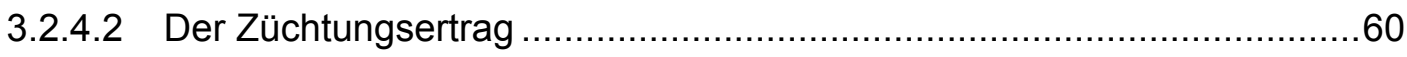

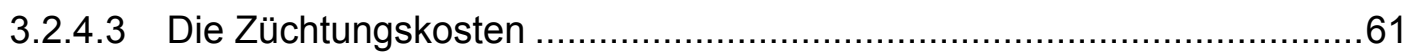

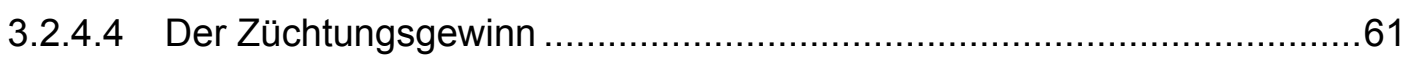

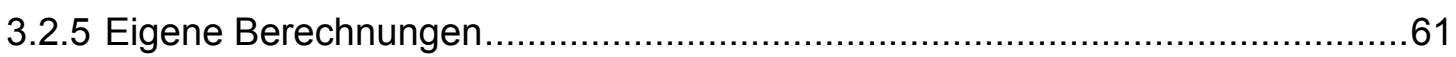

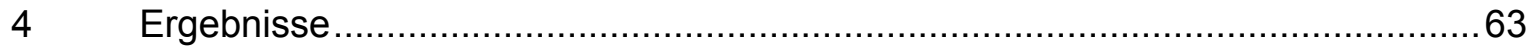

4.1 Ergebnisse der Datenauswertung ökologischer Milchviehbetriebe ....................63

4.1.1 Analyse der genetischen und phänotypischen Trends der Leistungs- und

funktionalen Merkmale bei Bullen und Kühen.....

4.1.1.1 Genetische Trends der Leistungsmerkmale bei Bullen der ökologischen und konventionellen Milchviehzucht.

4.1.1.2 Genetische Trends in den funktionalen Merkmalen bei Bullen der ökologischen und konventionellen Milchviehzucht. 67

4.1.1.3 Genetischer Trend bei Ökokühen .......................................................68

4.1.1.4 Phänotypischer Trend der Leistungsmerkmale bei Ökokühen ...............70

4.1.1.5 Phänotypischer Trend der funktionalen Merkmale bei Ökokühen ...........71

4.1.2 Genetisches Niveau und ET-Status der eingesetzten Bullen auf

ökologischen Milchviehbetrieben in Deutschland ..........................................74

4.1.2.1 ET-Status der Kuhväter und Anpaarungsbullen...................................75

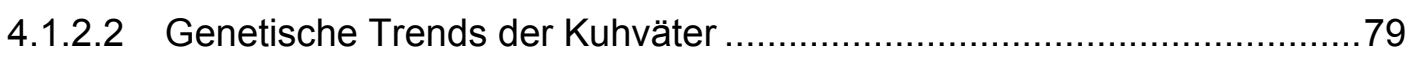

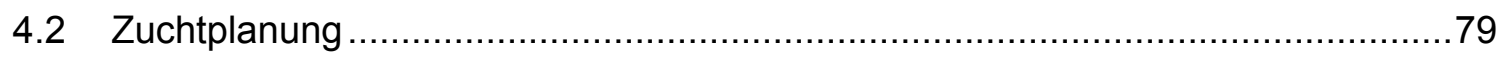

4.2.1 Interpretation der Ergebnisse aus dem Basislauf.......................................... 80

4.2.1.1 Variation im Testanteil und der Anzahl an Testbullen ...........................82

4.2.1.2 Veränderung der ökonomischen Gewichte in den Merkmalsgruppen Milch und Fitness ................................................ 88

4.2.1.3 Veränderung der Populationsgröße im ökologischen Zuchtprogramm ......................................................................

4.2.1.4 Töchterleistung pro Anzahl Besamungen .........................................94

4.2.1.5 Variation des Verhältnisses der Anteile an künstlicher Besamung in der Zucht- und Produktionsstufe

4.2.1.6 Unterschiede der Verteilung der Kühe auf die Zucht- und Produktionsstufe .96

4.2.1.7 Alter der Kühe bei Geburt der ersten Nachkommen .97

4.2.2 Ergebnisse der Untersuchungen zu Genotyp-Umwelt-Interaktionen im

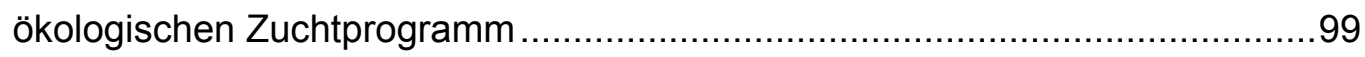

4.2.2.1 Genotyp-Umwelt-Interaktionen beim Merkmal Eiweißmenge . .99 
4.2.2.2 Genotyp-Umwelt-Interaktionen beim Merkmal Zellzahl ......................102

4.2.2.3 Genotyp-Umwelt-Interaktionen beim Merkmal Nutzungsdauer ............108

$5 \quad$ Diskussion

5.1 Genetische und phänotypische Trends für Leistungsmerkmale und funktionale Merkmale

5.1.1 Genetische Trends der Leistungsmerkmale bei Ökobullen und konventionellen Bullen

5.1.2 Genetische Trends in den funktionalen Merkmale bei Ökobullen und konventionellen Bullen

5.1.3 Genetische Trends bei Ökokühen.

5.1.4 Phänotypische Trends der Leistungsmerkmale bei Ökokühen

5.1.5 Phänotypische Trends in den funktionalen Merkmalen bei Ökokühen 118

5.2 ET-Status und genetisches Niveau der Ökobullen .........................................120

5.3 Diskussion der in ZPLAN variierten Parameter ...............................................122

5.3.1 Veränderung der Anzahl Testbullen und des Testanteils..............................122

5.3.2 Verschiebung der Gewichtung der Fitnessmerkmale................................. 123

5.3.3 Einfluss unterschiedlicher Populationsgrößen .......................................... 124

5.3.4 Variation der Töchterleistung pro Anzahl Testbesamungen .......................... 124

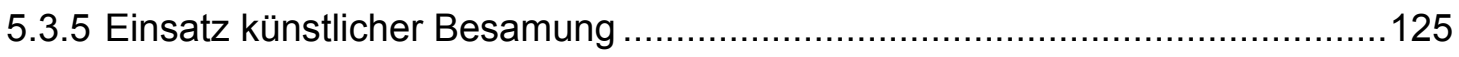

5.3.6 Verteilung der Kühe auf die Zucht- und Produktionsstufe..............................126

5.3.7 Alter der Kühe bei Geburt der ersten Nachkommen ................................... 127

5.3.8 Auswirkungen der Genotyp-Umwelt-Interaktionen.......................................127

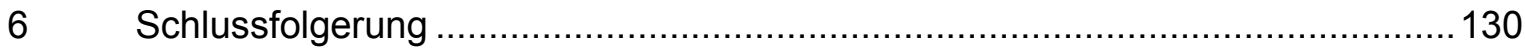

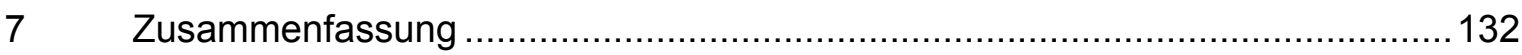

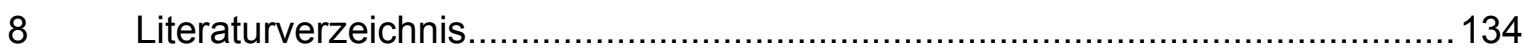




\section{Abbildungsverzeichnis}

Abbildung 1: Bestimmungsfaktoren zur Zuchtzielfestsetzung (Essl, 1999).

Abbildung 2: Zuchtwerttrend der Besamungsbullen der Rasse Schwarzbunt nach der VIT-Zuchtwertschätzung Mai 2006 (VIT, 2007).

Abbildung 3: Regionale Verteilung der Ökokühe in Deutschland (Nieberg und

Deeken, 2005) .................................................................................. 30

Abbildung 4: Darstellung des Rotationsanpaarungsmodells (Postler, 2002b)................... 33

Abbildung 5: Datenmaterial zur Analyse des Status der auf ökologischen Betrieben eingesetzten Kuhväter.

Abbildung 6: Genetischer Trendvergleich der mittleren Zuchtwerte für das Merkmal Milch-kg.

Abbildung 7: Genetischer Trendvergleich der mittleren Zuchtwerte für das

Merkmal Fett-\%

Abbildung 8: Genetischer Trendvergleich der mittleren Zuchtwerte für das

Merkmal Eiweiß-\%.

Abbildung 9: Genetischer Trendvergleich der mittleren Zuchtwerte für das Merkmal Fett-kg.

Abbildung 10: Genetischer Trendvergleich der mittleren Zuchtwerte für das Merkmal Eiweiß-kg.

Abbildung 11: Genetischer Trend in den Zuchtwerten Milch-kg, Fett-kg und

Eiweiß-kg bei Ökokühen.

Abbildung 12: Genetischer Trend in den Zuchtwerten Fett- und Eiweiß-\% bei

Ökokühen.

Abbildung 13: Phänotypischer Trend für das Merkmal Zellzahl in verschiedenen

Laktationen der Ökokühe

Abbildung 14: Phänotypischer Trend für das Merkmal Zwischenkalbezeit in verschiedenen Laktationen der Ökokühe.

Abbildung 15: Phänotypischer Trend für das Merkmal Erstkalbealter bei erstlaktierenden Ökokühen.

Abbildung 16: ET-Status der Kuhväter bei Betrachtung aller Rassen. 75

Abbildung 17: ET-Status der Kuhväter bei Betrachtung der Rasse Holstein.

Abbildung 18: Status der Kuhväter (Deckbulle/Testbulle/zuchtwertgeprüfter Bulle) bei Betrachtung aller Rassen.

Abbildung 19: Status der Kuhväter (Deckbulle/Testbulle/zuchtwertgeprüfter Bulle) bei Betrachtung der Rasse Holstein. 77

Abbildung 20: ET-Status des Anpaarungsbullen bei Betrachtung aller Kuhrassen. 
Abbildung 21: Status der Anpaarungsbullen (Deckbulle/Testbulle/zuchtwertgeprüfter Bulle) bei Betrachtung aller Rassen.

Abbildung 22: Trendvergleich zwischen Nicht-ET - und ET-Kuhvätern des Geburtsjahres 2000.

Abbildung 23: Prozentuale Veränderung der naturalen Zuchtfortschritte der Merkmale bei Veränderung des Testanteils von $25 \%$ auf $50 \%$.

Abbildung 24: Auswirkungen auf den Züchtungsgewinn ( $€ /$ Kuh/Jahr) bei einer unterschiedlichen Anzahl an Testbullen und variiertem Testanteil im Vergleich zur Ausgangssituation mit 20 Testbullen und einem Testanteil von 0,25.

Abbildung 25: Relativer Anteil der Merkmalsgruppen am gesamten monetären Zuchtfortschritt pro Jahr bei Verringerung bzw. Erhöhung der wirtschaftlichen Gewichte der Fitnessmerkmale.

Abbildung 26: Prozentuale Veränderung der naturalen Zuchtfortschritte der einzelnen Merkmale bei Erhöhung der Populationsgröße von 109.600 auf 200.000 Tiere.

Abbildung 27: Vergleich des Züchtungsertrags $(€)$, des Züchtungsgewinns (€) und der Züchtungskosten ( $€$ ) bei unterschiedlicher Populationsgröße.

Abbildung 28: Variation der Anzahl Besamungen pro Töchterleistung und die sich dadurch ergebenden Unterschiede im monetären Zuchtfortschritt pro Jahr insgesamt $(€)$ und dem Züchtungsgewinn $(€)$ im Vergleich zum Ausgangsverhältnis

Abbildung 29: Auswirkungen auf den Züchtungsgewinn (€), den Züchtungsertrag $(€)$, den gesamten monetären Zuchtfortschritt pro Jahr $(€)$ und den Züchtungskosten $(€)$ bei unterschiedlichen Anteilen der künstlichen Besamung (\%) sowohl in der Zuchtstufe als auch in der Produktionsstufe (ZS:PS).

Abbildung 30: Züchtungsgewinns $(€)$ in Abhängigkeit vom durchschnittlichen

Erstkalbealter. 98

Abbildung 31: Züchtungsertrag (€) für das Merkmal Zellzahl in unterschiedlichen Zuchtprogrammen mit verschiedenen genetischen Korrelationen (rg) und $\mathrm{h}^{2}=0,34$ 100

Abbildung 32: Naturaler Zuchtfortschritt für das Merkmal Eiweiß-kg bei $h^{2}=0,34$. 101

Abbildung 33: Züchtungsertrag (€) für das Merkmal Eiweiß-kg in unterschiedlichen Zuchtprogrammen mit verschiedenen genetischen Korrelationen ( $\mathrm{rg}$ ) und $\mathrm{h}^{2}=0,25$. 101

Abbildung 34: Naturaler Zuchtfortschritt für das Merkmal Eiweiß-kg bei $h^{2}=0,25$ 102 
Abbildung 35: Züchtungsertrag (€) für das Merkmal Zellzahl in unterschiedlichen Zuchtprogrammen mit verschiedenen genetischen Korrelationen ( $\mathrm{rg}$ ) und $\mathrm{h}^{2}=0,12$.

Abbildung 36: Naturaler Zuchtfortschritt für das Merkmal Zellzahl bei $h^{2}=0,12$. 104

Abbildung 37: Züchtungsertrag (€) für das Merkmal Zellzahl in unterschiedlichen Zuchtprogrammen mit verschiedenen genetischen Korrelationen (rg) und $h^{2}=0,08$. 105

Abbildung 38: Naturaler Zuchtfortschritt für das Merkmal Zellzahl bei $h^{2}=0,08$. 105

Abbildung 39: Züchtungsertrag ( $€$ ) für das Merkmal Zellzahl in unterschiedlichen Zuchtprogrammen mit verschiedenen genetischen Korrelationen ( $\mathrm{rg}$ ) und $\mathrm{h}^{2}=0,25$. 106

Abbildung 40: Naturaler Zuchtfortschritt für das Merkmal Zellzahl bei $h^{2}=0,25$. 107

Abbildung 41: Züchtungsertrag (€) für das Merkmal Nutzungsdauer in unterschiedlichen Zuchtprogrammen mit verschiedenen genetischen Korrelationen $(\mathrm{rg})$ und $\mathrm{h}^{2}=0,12$ 108

Abbildung 42: Naturaler Zuchtfortschritt für das Merkmal Nutzungsdauer bei $h^{2}=0,12$ 109

Abbildung 43: Züchtungsertrag (€) für das Merkmal Nutzungsdauer in unterschiedlichen Zuchtprogrammen mit verschiedenen genetischen Korrelationen $(\mathrm{rg})$ und $\mathrm{h}^{2}=0,08$.

Abbildung 44: Naturaler Zuchtfortschritt für das Merkmal Nutzungsdauer bei $h^{2}=0,08$

Abbildung 45: Züchtungsertrag (€) für das Merkmal Nutzungsdauer in unterschiedlichen Zuchtprogrammen mit verschiedenen genetischen Korrelationen (rg) und $\mathrm{h}^{2}=0,25$

Abbildung 46: Naturaler Zuchtfortschritt für das Merkmal Nutzungsdauer bei $h^{2}=0,25$ 


\section{Tabellenverzeichnis}

Tabelle 1: Der Anteil der einzelnen Merkmalsbereiche am monetären

Zuchtfortschritt (Krogmeier et al., 2007).

Tabelle 2: Genetische Korrelationen $\left(r_{g}\right)$ für die Milch-, Fett- und Eiweißmenge............... 40

Tabelle 3: Genetische Korrelationen $\left(r_{g}\right)$ für Fett-, Eiweißgehalt und Zellzahl.

Tabelle 4: Vorläufige Ergebnisse der Genotyp-Umwelt-Interaktion zwischen konventionellen und ökologischen Betrieben in der Schweiz (Bapst und Stricker, 2006).

Tabelle 5: Vorläufige Ergebnisse der Genotyp-Umwelt-Interaktion zwischen konventionellen und ökologischen Betrieben in Deutschland (Reinhardt, 2006).

Tabelle 6: Darstellung des gesamten Datenmaterials und die Auswahl der zur Berechnung geeigneten Daten.

Tabelle 7: Die wichtigsten Parameter zur Beschreibung der Populationsstruktur im ökologischen Basiszuchtprogramm.

Tabelle 8: Die wichtigsten biologisch-technischen Parameter für den Basislauf des ökologischen Zuchtprogramms.

Tabelle 9: Die wichtigsten Kostenparameter für den Basislauf des

Zuchtprogramms für die ökologische Milchviehhaltung. 51

Tabelle 10: Investitionsparameter des Basiszuchtprogramms.

Tabelle 11: Darstellung der 19 verschiedenen Selektionsgruppen und Genflüsse zur Übertragung des genetischen Fortschritts.

Tabelle 12: Darstellung der Genflüsse in den 19 Selektionsgruppen.

Tabelle 13: Phänotypische (oberhalb der Diagonalen) und genetische Korrelationen (unterhalb der Diagonalen) und Heritabilitäten (Diagonale) der Merkmale im Index.

Tabelle 14: Wirtschaftliche Gewichte für Milchleistungs- und funktionale Merkmale (Lind, 2006). 56

Tabelle 15: Informationsquellen für die ZWS der HK-ö (Herdbuchkühe der ökologischen Zuchtstufe), deren Merkmale und Anzahl an wiederholten Messungen.

Tabelle 16: Informationsquellen für die ZWS der TB-ö (Testbullen der ökologischer Zuchtstufe zum Zeitpunkt der Anpaarung), deren Merkmale und Anzahl an wiederholten Messungen.

Tabelle 17: Durchschnittliche Veränderung (Regressionskoeffizienten) der Merkmale Kalbeverlauf, Totgeburten und NNR 90. 
Tabelle 18: Durchschnittliche Veränderung (Regressionskoeffizienten) der

Relativzuchtwerte pro Jahr für Ökobullen im Vergleich zu den

konventionellen Bullen.

Tabelle 19: Durchschnittliche Veränderung der Relativzuchtwerte

(Regressionskoeffizienten) pro Jahr bei den Ökokühen

Tabelle 20: Durchschnittliche Veränderung (Regressionskoeffizienten) pro Jahr für die Einsatzleistung Milch-kg, Fett-kg, Eiweiß-kg, Fett-\% und Eiweiß-\% der Ökokühe in der 1., 2. und > 2. Laktation.

Tabelle 21: Monetärer Zuchtfortschritt pro Jahr gesamt (€), Züchtungsertrag (€), Züchtungskosten $(€)$ und Züchtungsgewinn (€) nach den ersten Berechnungen für das Zuchtprogramm der Basispopulation und einer Investitionsperiode von 20 Jahren.

Tabelle 22: Naturaler und monetärer Zuchtfortschritt der Merkmale pro Jahr (standardisierter nat ZF/Jahr (in $\mathrm{s}_{\mathrm{A}}$ * 100 ), in \% bzw. mon ZF/Jahr (€), absoluter und relativer Anteil der Merkmalsgruppen Milch und Fitness am gesamten monetären Zuchtfortschritt pro Jahr (monZF/Jahr gesamt, $€$ ) für die Basispopulation

Tabelle 23: Einfluss unterschiedlicher Testanteile auf den naturalen Zuchtfortschritt (in $\mathrm{s}_{\mathrm{A}}$ * 100) und den gesamten monetären Zuchtfortschritt pro Jahr (€) im Vergleich zum Basisszenario (fett gedruckt).

Tabelle 24: Vergleich von monetärem Zuchtfortschritt pro Jahr insgesamt (€), Züchtungskosten $(€)$ und dem Züchtungsgewinn $(€)$ und den jeweiligen relativen Unterschieden (\%) zum Basisszenario (fett gedruckt) bei verschiedenen Testanteilen.

Tabelle 25: Auswirkung auf monetären Zuchtfortschritt pro Jahr insgesamt $(€)$, Züchtungskosten $(€)$ und dem Züchtungsgewinn $(€)$ und relative Unterschiede (\%) zum Basisszenario (fett gedruckt) bei Variation der Anzahl der Testbullen.

Tabelle 26: Verringerung und Erhöhung der wirtschaftlichen Gewichte der Fitnessmerkmale um jeweils $25 \%$ und $50 \%$ von den Ausgangsgewichten (100\%).

Tabelle 27: Naturaler Zuchtfortschritt pro Jahr (in $\mathrm{s}_{\mathrm{A}}$ * 100) und monetärer Zuchtfortschritt pro Jahr gesamt der Fitnessmerkmale (monZF/Jahr gesamt, €) nach Verringerung bzw. Erhöhung der wirtschaftlichen Gewichte der Fitnessmerkmale um jeweils $25 \%$ und $50 \%$ im Vergleich zum Basisszenario (fett gedruckt). 
Tabelle 28: Monetärer Zuchtfortschritt pro Jahr der Merkmalsgruppen Milch und Fitness bei Verringerung bzw. Erhöhung der wirtschaftlichen Gewichte der Fitness-merkmale um jeweils $25 \%$ und $50 \%$; Relativer Anteil einer Merkmalgruppe am gesamten monetären Zuchtfortschritt pro Jahr (€)

Tabelle 29: Einfluss verschiedener Größen der ökologischen Milchviehpopulation auf den naturalen Zuchtfortschritt (in $\mathrm{s}_{\mathrm{A}}$ * 100) und den gesamten monetären Zuchtforschritt pro Jahr $(€)$ im Vergleich zur Ausgangspopulation (fett gedruckt). 92

Tabelle 30: Unterschiedliche Verteilung der Anzahl Kühe in der Zucht- und Produktionsstufe (in \%) und die Auswirkung auf den monetären Zuchtfortschritt pro Jahr gesamt (€), den Züchtungsertrag (€), die Züchtungskosten $(€)$ und den Züchtungsgewinn $(€)$ im Vergleich zum Basisszenario (fett gedruckt).

Tabelle 31: Durchschnittliches Alter der Kühe bei der Geburt der ersten Nachkommen (Jahre). Vergleich des monetären Zuchtfortschrittes pro Jahr insgesamt $(€)$, des Züchtungsertrags $(€)$, der Züchtungskosten ( $€$ ) und des Züchtungsgewinns $(€)$ im Vergleich zum Basisszenario (fett gedruckt). 


\section{Verzeichnis der Abkürzungen}

Abb.

ADR

AGN

AGÖL

ALL

ANLS

AÖLZ

ARGE

Art.

BLE

BLUP

BMELV

BÖLW

bzgl.

bzw.

ct

d

DESTATIS

DHV

DMG

E-kg

ET

et al.

$€$

FAO

FE

$\mathrm{FiBL}$

F-kg

FRUm

FRUp

FW

GZ-LL

$h^{2}$
Abbildung

Arbeitsgemeinschaft Deutscher Rinderzüchter

Applied Genetics Network

Arbeitsgemeinschaft ökologischer Landbau

Arbeitsgemeinschaft Lebenslinien

Arbeitsgemeinschaft Natürlicher Landbau und Siedlung

Arbeitsgemeinschaft Österreichischer Lebensleistungszüchter

Arbeitsgemeinschaft für Rinderzucht auf Lebensleistung

Artikel

Bundesanstalt für Landwirtschaft und Ernährung

Best Linear Unbiased Prediction - Beste Lineare Unverzerrte

Vorhersage

Bundesministerium für Ernährung, Landwirtschaft und

Verbraucherschutz

Bund ökologischer Lebensmittelwirtschaft

bezüglich

beziehungsweise

Cent

Tag

Statistisches Bundesamt Deutschland

Deutscher Holstein Verband

Durchschnittliches Minutengemelk

Eiweiß Kilogramm

Embryotransfer

et altera

Euro

Food and Agriculture Organization of the United Nations

Form und Euter

Forschungsinstitut für biologischen Landbau

Fett Kilogramm

Fruchtbarkeit maternal

Fruchtbarkeit paternal

Fleischwert

Gesamtzuchtwert-Lebensleistung

Heritabilität 


\begin{tabular}{|c|c|}
\hline ha & Hektar \\
\hline $\mathrm{HF}$ & Holstein-Friesian \\
\hline IFOAM & International Federation of Organic Agriculture Movement \\
\hline INTERBULL & International Bull Evaluation Service \\
\hline $\mathrm{KB}$ & künstliche Besamung \\
\hline $\mathrm{Kg}$ & Kilogramm \\
\hline $\mathrm{km}^{2}$ & Quadratkilometer \\
\hline $\mathrm{KV}$ & Kalbung und Vitalität \\
\hline KVLm & Kalbeverlauf maternal \\
\hline KVLp & Kalbeverlauf paternal \\
\hline I & Liter \\
\hline Mio & Million \\
\hline $\mathrm{ml}$ & Milliliter \\
\hline MLP & Milchleistungsprüfung \\
\hline MOET & Multiple Ovulation and Embryo Transfer \\
\hline ND & Nutzungsdauer \\
\hline NRR 90 & Non Return Rate 90 Tage \\
\hline ÖMW & Ökologischer Milchwert \\
\hline ÖZW & Ökologischer Gesamtzuchtwert \\
\hline Pers & Persistenz \\
\hline PL & Persistenz und Leistungssteigerung \\
\hline RBB & Rinderproduktion Berlin-Brandenburg $\mathrm{GmbH}$ \\
\hline $\mathrm{rg}$ & genetische Korrelation \\
\hline RZE & Relativzuchtwert Exterieur (lineare Beschreibung, Beurteilung) \\
\hline RMV & Rinderzuchtverband Mecklenburg-Vorpommern GmbH \\
\hline $\mathrm{RSH}$ & Rinderzucht Schleswig-Holstein e. G. \\
\hline RZG & Gesamtzuchtwert \\
\hline RZM & $\begin{array}{l}\text { Relativzuchtwert Milchleistung (Fett-kg, Eiweiß-kg, Fett-\%, } \\
\text { Eiweiß-\%) }\end{array}$ \\
\hline RZN & Relativzuchtwert funktionale Nutzungsdauer \\
\hline RZS & Relativzuchtwert Zellzahl (somatischer Zellgehalt) \\
\hline $\mathrm{RZZ}$ & Relativzuchtwert Zuchtleistung (Kalbeverlauf, Totgeburten, NRR 90) \\
\hline SOEL & Stiftung Ökologie und Landbau \\
\hline $\mathrm{t}$ & Tonne \\
\hline Tab. & Tabelle \\
\hline TOTm & Totgeburt maternal \\
\hline TOTр & Totgeburt paternal \\
\hline
\end{tabular}




$\begin{array}{ll}\text { u. a. } & \text { unter anderem } \\ \text { usw. } & \text { und so weiter } \\ \text { v. a. } & \text { vor allem } \\ \text { vgl. } & \text { vergleiche } \\ \text { VIT } & \text { Vereinigte Informationssysteme Tierhaltung w. V. } \\ \text { vs. } & \text { versus } \\ \text { z. B. } & \text { zum Bespiel } \\ \text { ZEH } & \text { Zuchtrinder-Erzeugergemeinschaft Hannover e. G. } \\ \text { z. T. } & \text { zum Teil } \\ \text { ZUCHTDATA } & \text { Zuchtwertdatenbank Österreich } \\ \text { ZW } & \text { Zuchtwert } \\ \text { ZWS } & \text { Zuchtwertschätzung } \\ \text { ZZ } & \text { Zellzahl }\end{array}$




\section{Einleitung}

Beeinflusst durch die vermehrte Nachfrage des Verbrauchers nach Produkten aus dem ökologischen Landbau, und aufgrund staatlicher Förderprogramme für tiergerechte Haltungssysteme hat in den vergangenen Jahren die Zahl der ökologisch wirtschaftenden Betriebe in Deutschland zugenommen. Unter Einhaltung des Ziels der tiergerechten Haltung und Fütterung wird in der ökologischen Milchviehhaltung neben der Tiergesundheit und Leistungsstabilität auch eine optimale Qualität tierischer Erzeugnisse angestrebt. Dabei unterliegt diese Milchproduktion im Vergleich zur konventionellen Produktionsweise wesentlich strengeren Anforderungen an die Zucht und Haltung der Kühe. Eine Anpassung züchterischer Konzepte an die jeweiligen spezifischen Bedingungen ist zu diesem Zweck notwendig.

Basierend auf an aktuellen Daten geschätzten Parametern und unter Zugrundelegung der aktuellen und für die Zukunft absehbaren Strukturen und Szenarien der ökologischen Milchviehhaltung in Deutschland, sollen in dieser Arbeit verschiedene Varianten ökologischer Zuchtprogramme modelliert und verglichen werden. Zielgröße ist dabei der erwartete Zuchtfortschritt im ökologischen Gesamtzuchtwert und in den für die ökologische Milchviehzucht bedeutsamen funktionalen Merkmalen. Zusätzlich sollen einige Nebenbedingungen, wie der Verzicht auf den Einsatz künstlicher Besamung, den Planungsrechnungen zu Grunde gelegt und daraus quantifiziert werden, welche Kosten im Sinn des Verzichts auf möglichen Zuchtfortschritt mit diesen Nebenbedingungen einhergehen.

Das Ziel dieser Arbeit liegt darin, möglichst realistische und praxisrelevante Szenarien zu entwickeln und unter den gegebenen Rahmenbedingungen und Zieldefinitionen die effizienteste Zuchtstrategie für ökologisch wirtschaftende Milchviehbetriebe zu identifizieren. Anhand der in Deutschland vorliegenden genetischen und phänotypischen Trends soll der Stand der ökologischen Milchviehpopulation untersucht werden bzw. die Selektionskriterien in der praktischen Umsetzung ermittelt werden. Außerdem wird weiter überprüft, wie der Verzicht auf Embryotransfer bzw. das Verbot des Einsatzes von Zuchttieren die aus Embryotransfer stammen in der Praxis umgesetzt wird. Die Ergebnisse dieser Arbeit sollen als Grundlage für die Akzeptanz und die Implementierung effizienter Zuchtprogramme im Bereich der ökologischen Milchviehhaltung dienen.

Nach einer Einführung der in der Literatur beschriebenen Grundlagen der ökologischen Milchviehhaltung und bereits bestehender Zuchtprojekte, werden das untersuchte Datenmaterial und die Auswertungsmethodik dargestellt. Im ersten Teil der eigenen Untersu- 
chungen werden auf Basis von Zuchtwertdaten ökologischer und konventioneller Milchviehbetriebe die genetischen und phänotypischen Trends in den Leistungsmerkmalen und funktionalen Merkmalen bei Kühen und Bullen ausgewertet. Zusätzlich wird der Einsatz von aus Embryotransfer abstammenden Kuhvätern und Anpaarungsbullen ermittelt. Im zweiten Teil werden verschiedene Zuchtplanungsberechnungen zur Untersuchung des möglichen Zuchtfortschritts eines ökologischen Zuchtprogramms durchgeführt. Alle ermittelten Ergebnisse werden im darauf folgenden Diskussionsteil näher erläutert und mit den Ergebnissen aus der Wissenschaft diskutiert. Im Anschluss daran werden für die wichtigsten Ergebnisse der einzelnen Themenblöcke Schlussfolgerungen formuliert. Zum Abschluss wird die Arbeit in einer kurzen Zusammenfassung dargestellt. 


\section{Literaturübersicht}

\subsection{Organisation der Rinderzucht in Deutschland}

Basierend auf den in den Jahren 1837 bis 1844 in einzelnen Regionen gegründeten „Vereinen zur Beförderung und Verbesserung der Pferde- und Rinderzucht" und den Gründungen der ersten Tierschauvereine im Jahr 1845, wurde im Jahr 1868 die organisierte Tierzucht im landwirtschaftlichen Bereich eingeführt (Grandke, 2002; Walter, 2003). Ausgelöst durch Einfuhrsperren und der sich daraus ergebenen Intensivierung des Reinzuchtgedankens bei deutschen Rinderrassen entwickelte sich die so genannte Herdbuchidee. Durch die steigende Nachfrage nach dem besten und milchreichsten Vieh, durch hohe Kaufgebote bei sicheren Abstammungsnachweisen, aber auch um den Wünschen ausländischer Käufer entgegen zu kommen, wurden schließlich Herdbücher eingerichtet. Neben England (1922), den USA (1871, 1877 und 1885), den Niederlanden (1874 und 1879) und Dänemark (1881), wurde im Jahr 1876 auch in Deutschland die erste Herdbuchzucht gegründet (Grothe, 1993; Walter, 2003). Weitere deutsche Herdbuchorganisationen wurden in den darauf folgenden Jahren gegründet. Bei der Bestimmung eines Standardtyps für jede Rasse spielten zu der Zeit die Exterieurmerkmale eine größere Rolle als die Leistung. Dieser Merkmalskomplex gewann erst durch die Entwicklung von Leistungsprüfungen, als Basis für die Selektion, zu Beginn des 20. Jahrhunderts mehr an Bedeutung (Kräusslich, 1994).

Einige der damals gegründeten Organisationen, sind heute als Zuchtorganisationen vorzufinden. Mittlerweile gehören die derzeitigen Verbände der Deutschen Holstein Zucht z. T. größeren Zusammenschlüssen an. So besteht zum einen das „TopQ“ - Zuchtprogramm aus dem Landesverband Thüringer Rinderzüchter (LTR), dem Rinderzuchtverband Sachsen-Anhalt (RSA), der Rinderunion West (RUW), der Weser-Ems-Union (WEU) und der Zucht- und Besamungsunion Hessen (ZBH) (TopQ, 2007). Zum anderen führt die „NOG - Nord-Ost-Genetik“ mit den Partnern Rinderzucht Schleswig-Holstein (RSH), Rinderzucht Mecklenburg-Vorpommern $\mathrm{GmbH}$ (RMV), der Rinderproduktion BerlinBrandenburg GmbH (RBB) und mit Masterrind GmbH seit 1998 ein gemeinsames Zuchtprogramm durch (NOG, 2007). Neben dem Großteil an konventionellen Milchviehbetrieben gehören auch ökologisch wirtschaftende Milchviehbetriebe diesen Zuchtverbänden an. Der Deutsche Holstein Verband (DHV) fungiert als Dachverband aller deutschen Holsteinzuchtverbände. Für die übrigen Rassen sind in Deutschland die Arbeitsgemeinschaft Süddeutscher Rinderzucht- und Besamungsorganisationen e. V. (ASR) und der Bundesverband Deutscher Fleischrinderzüchter und -halter e. V. (BDF) als weitere Rassendach- 
verbände tätig. Die Interessen der organisierten Rinderzucht in Deutschland werden auf nationaler und internationaler Ebene durch die Arbeitsgemeinschaft Deutscher Rinderzüchter e. V. (ADR) vertreten.

\subsubsection{Zuchtziel}

Das nach Fewson (1993) für die allgemeine konventionelle Milchviehzucht definierte Zuchtziel ist die Züchtung von vitalen Tieren, die unter künftigen Produktionsbedingungen einen höchstmöglichen Gewinn garantieren.

Essl (1999) übt etwas Kritik an der von Fewson (1993) vorgeschlagenen Zuchtzieldefinition aus. Eine Beschränkung auf den ökonomischen Ansatz als Basis des Zuchtziels reicht seiner Meinung nach nicht aus, um eine verantwortungsvolle Zucht umsetzen zu können. Demzufolge sollten neben der bereits von Fewson (1993) erwähnten Beachtung biologischer Konsequenzen für die Langlebigkeit und Fruchtbarkeit, weitere wichtige Aspekte bei der Zuchtzielformulierung mit berücksichtigt werden. Nach Essl (1999) wirken die in Abbildung 1 aufgeführten Bestimmungsfaktoren auf das Zuchtziel ein.

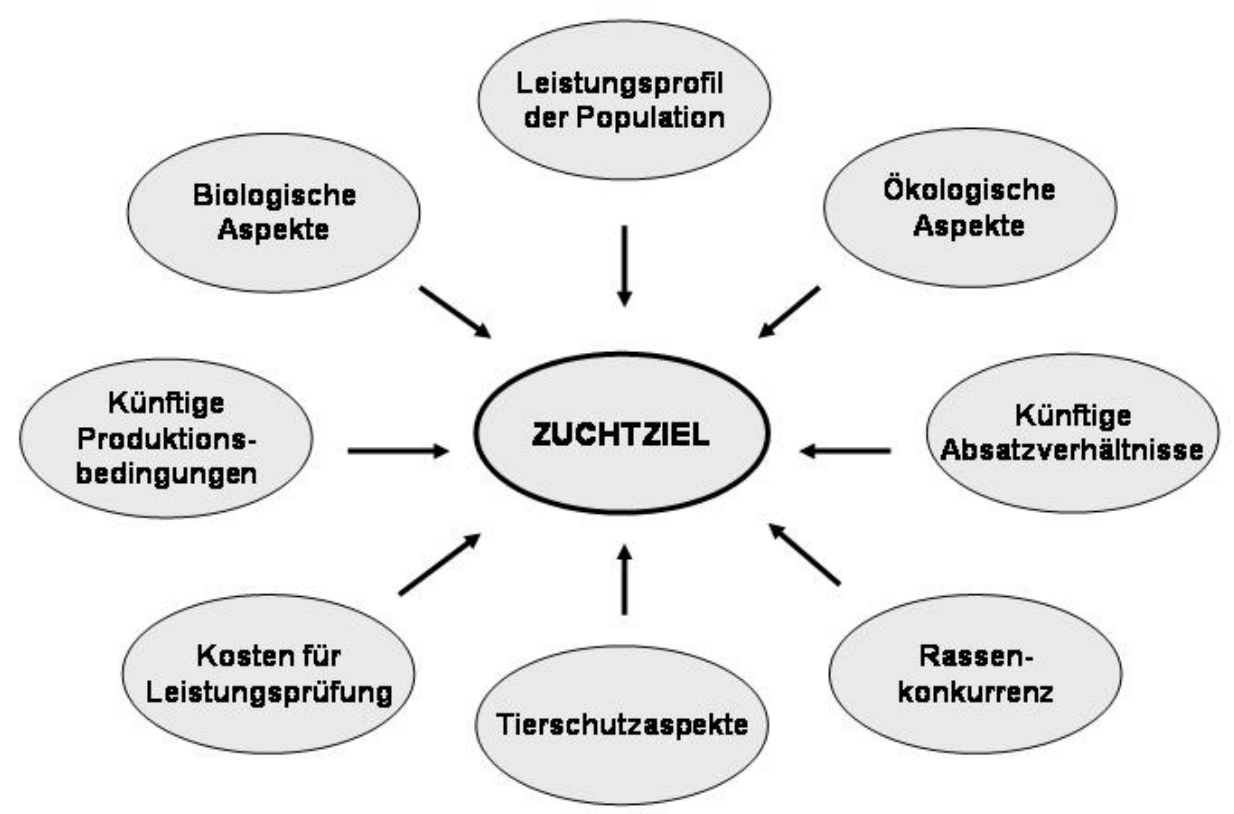

Abbildung 1: Bestimmungsfaktoren zur Zuchtzielfestsetzung (Essl, 1999). 
In diesem Zusammenhang werden nach Baumung (2006) bei der Zuchtzielformulierung zwei Ansätze, ein biologischer und ein ökonomischer, unterschieden. Der biologische Ansatz berücksichtigt nur die Merkmale, die unabhängig von Produktionsbedingungen, Kosten und Erlösen zu verbessern sind. Ergänzend zu Essl (1999) betont auch Baumung (2006), dass bei diesem Ansatz ethische Überlegungen eine entscheidende Rolle spielen. Mit dem zweiten Ansatz, dem ökonomischen Ansatz, wird die Zucht nach der wirtschaftlichen Bedeutung der Merkmale beschrieben. Hierbei sind Kosten und Erlöse entscheidende Einflussfaktoren.

Nach Grandke (2002) werden in der heutigen Milchviehzucht die Zuchtzielformulierungen von den Rassedachverbänden übernommen und dienen somit den Züchtern und Zuchtorganisationen als Orientierung für die Zuchtarbeit. Das heutige Zuchtziel in der konventionellen Milchviehhaltung ist bei der Rasse Deutsche Holsteins der Farbrichtung Schwarzbunt und Rotbunt speziell auf leistungsstarke, gesunde und langlebige Tiere ausgerichtet (DHV, 2007). Gewünscht wird eine rentable Leistungskuh im milchbetonten Typ und hohen Milchleistungen. Zudem soll die Kuh in Kombination mit einem großen Futteraufnahmevermögen, einer stabilen Gesundheit und einer guten Fruchtbarkeit über viele Laktationen nutzbar sein. Das genetische Leistungspotential liegt nach konkreten Vorstellungen der Holsteinzucht bei 10.000 kg Milch mit einem Fettgehalt von $4 \%$ und einem Eiweißgehalt von 3,4 \%. Der Körperbau mit einer maximalen Kreuzhöhe von 145 bis $156 \mathrm{~cm}$ und einem Gewicht von 650 bis 750 kg und die Bewegungsmechanik müssen den hohen Leistungsanforderungen entsprechen. Neben einem korrekten und widerstandsfähigen Fundament gilt ein gut melkbares Euter, das in Qualität und Funktionsfähigkeit den Anforderungen moderner Melksysteme entspricht, als Voraussetzung für hohe Dauerleistungen (DHV, 2007). Abgesehen von einer stärkeren Gewichtung des Merkmalkomplexes Fitness im Ökologischen Gesamtzuchtwert und einer Bevorzugung der Produktqualität anstelle der Quantität (Postler, 2002b), verfolgt die ökologische Milchviehzucht im Prinzip die gleichen Ziele wie die konventionelle Zucht.

\subsubsection{Zuchtprogramme beim Rind}

Zuchtprogramme verfolgen das Ziel, die Auswahl der besten Zuchttiere einer Generation zur Erstellung der nächsten Generation zu selektieren und den erzielten Zuchtfortschritt in die Produktionsstufe zu übertragen (Gierzinger, 2002). Ein erfolgreiches Zuchtprogramm umfasst nach Baumung (2006) entsprechend alle auch die auf zukünftige Bedingungen ausgerichteten Maßnahmen, die zur Umsetzung eines definierten Zuchtziels erforderlich sind. Ebenso müssen optimale Vermarktungswege für die Zuchtprodukte bestimmt wer- 
den. In Deutschland sind nach König (2001) die Größe der aktiven Zuchtpopulation, die Herdbuchdichte und die Formen der Leistungsprüfung grundlegende Faktoren zur effizienteren Gestaltung eines Zuchtprogramms.

Das Grundgerüst eines derzeitigen konventionellen Zuchtprogramms wurde von Skjervold (1966) entwickelt. Diese Zuchtprogramme sind nach Grandke (2002) gekennzeichnet durch gezielte Anpaarungen der besten nachkommengeprüften Bullen mit den Bullenmüttern, die Anpaarung selektierter Jungbullen mit Herdbuchkühen zur Erstellung von Nachkommen für die Nachkommenprüfung und durch Konservierung des Testbullenspermas während des Testeinsatzes und der Wartezeit. Selektion der Bullen aufgrund ihrer Töchterleistungen, der Einsatz von geprüften und selektierten Bullen als Kuhväter und die Selektion der besten Bullen als Bullenväter zur Anpaarung mit aktuellen Bullenmüttern zur Erzeugung der nächsten Generation sind weitere Charakteristika, die das Basiszuchtprogramm nach Skjervold (1966) beschreiben.

Weitere Zuchtprogramme und der Einsatz alternativer Biotechnologien bauen auf diesem KB-Zuchtprogramm auf. Als Beispiel für alternative Zuchtprogramme sind hier Nukleuszuchtprogramme zu nennen. Mit diesen Programmen kann der Umfang der Leistungsprüfung reduziert werden, Technologien zur Bestimmung von schwer zu erfassenden Merkmalen werden intensiviert, die vollständige Kontrolle des Zuchtablaufs untersteht einer zentralen Leitung und die Einführung von Biotechnologien, wie z. B. Embryotransfer, wird erleichtert (Willam, 1997). Die Kennzeichen von MOET-Nukleuszuchtprogrammen (Multiple Ovulation and Embryo Transfer) (Nicholas und Smith, 1983) werden in Anlehnung an Heckenberger (1991) und Willam (1997) kurz erläutert. Der Einsatz von Embryotransfer führt zu einer Erhöhung der Vermehrungsrate der Kühe, womit innen eine neue Bedeutung im Zuchtgeschehen zugeteilt wird. Sowohl die Selektionsintensität bei Bullenmüttern als auch bei den Kuhmüttern wird durch Embryotransfer erhöht. Außerdem können unter Verwendung dieser Technologie, Vollgeschwisterinformationen für die Zuchtwertschätzung genutzt werden.

Die Berücksichtigung von Ahnen- und Voll- bzw. Halbgeschwisterleistungen führt zu einer Verkürzung der Generationsintervalle. Weiterhin ist das MOET-Nukleuszuchtprogramm dadurch gekennzeichnet, dass auf Nachkommenprüfung ganz verzichtet oder eine Vorselektion der Wartebullen aufgrund der Geschwisterleistungen durchgeführt wird. Im Vergleich zum Geflügel und zu Schweinen werden Nukleuszuchtprogramme in der Rinderzucht aufgrund des hohen organisatorischen und finanziellen Aufwands weniger eingesetzt. 


\subsection{Genetische und phänotypische Trends}

Genetische Trends werden im Rahmen der routinemäßigen Zuchtwertschätzung im VIT Verden ermittelt. Anhand der mittleren Kuhzuchtwerte pro Geburtsjahrgang wird der jährliche genetische Fortschritt geschätzt. Damit können Schlussfolgerungen hinsichtlich des Standes der Population getroffen bzw. Selektionsschwerpunkte in Bezug auf einzelne Merkmale der Bullen und Kühe beobachtet werden (Swalve und Höver, 2003; Bergfeld, 2004). Die aktuellen genetischen Trends der Zuchtwertschätzung Mai 2006 für die Rassen Schwarzbunte und Rotbunte Holsteins, Rotvieh, Angler und Jersey sind im Jahresbericht 2006 dargestellt. Die Ergebnisse des VIT für den RZM, RZE, RZN, RZS und für Milch-, Fett- und Eiweiß-kg, sind in Abbildung 2 am Beispiel Schwarzbunter KB-Bullen dargestellt.
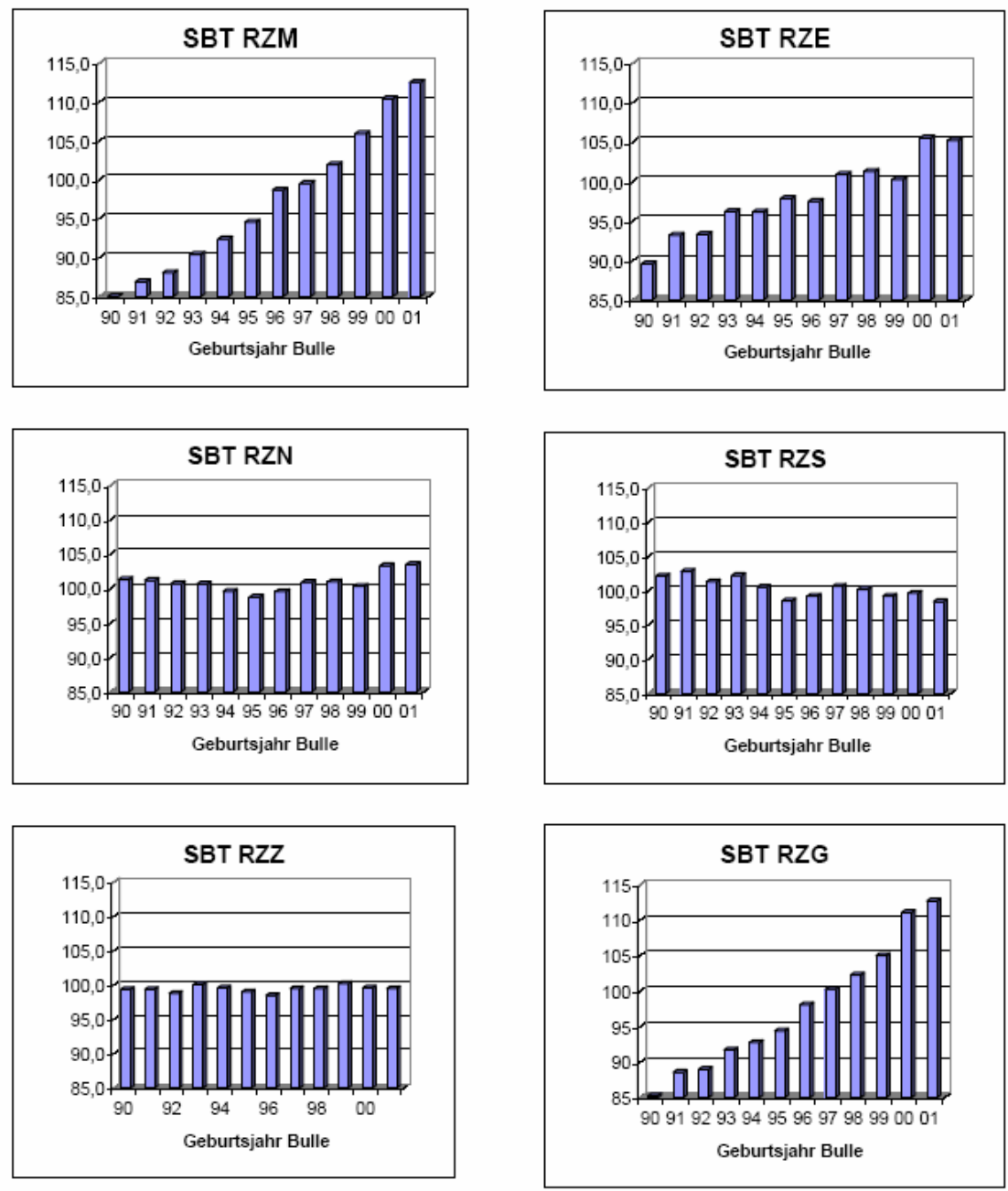

Abbildung 2: Zuchtwerttrend der Besamungsbullen der Rasse Schwarzbunt nach der VITZuchtwertschätzung Mai 2006 (VIT, 2007). 
Laut VIT (2006a) ist bei Holstein-Bullen der Zuchtfortschritt von 2 bis 3 RZG-Punkten mit dem Mittel der vergangenen Jahre zu vergleichen. Der Grund dafür liegt hauptsächlich bei der parallel laufenden Abschreibung des RZM. Die Abbildung 2 zeigt, bedingt durch einen schwachen Jahrgang 1998 und dem Wegfall des Jahrgangs 1995 aus der Basis, für den Relativzuchtwert Exterieur eine Abschreibung in Höhe von etwa 1 Punkt. Diese Basisabschreibung fällt im Vergleich zum Mittel der letzten Jahre geringer aus. Auch die Basisabschreibung von 0,9 für RZS lässt sich laut VIT (2006a) aufgrund des Wegfalls des Geburtsjahrgangs 1995 erklären. Da aber der mittlere genetische Trend für RZS über die letzten 10 Jahre leicht negativ ist, ist diese Entwicklung nicht als Zuchtfortschritt im langfristigen Sinn zu interpretieren. Der Zuchtfortschritt für den Relativzuchtwert funktionale Nutzungsdauer ist eher gering und wird durch eine entsprechende Basisabschreibung von 0,3 Punkte ausgedrückt (VIT, 2006a).

Die vom VIT ermittelten und im August 2006 veröffentlichten, mittleren Zuchtwerte Schwarzbunter KB-Bullen der Jahrgänge 1988 bis 2001 weisen auf einen deutlich positiven Trend in der Leistung und den Inhaltsstoffen hin (VIT, 2006b). Der mittlere Zuchtwert der Milchmenge liegt im Jahr 1988 bei -401 kg. Mit Ausnahme des Geburtsjahrgangs 1997 verbessert sich der Zuchtwert Milchleistung jährlich um mehr als $100 \mathrm{~kg}$ und sogar um 285 kg im Bullenjahrgang 2000. Im jüngsten Geburtsjahrgang 2001 wird ein mittlerer Zuchtwert von $1002 \mathrm{~kg}$ Milch erreicht, wobei für die Bullen dieses Jahrgangs und für das Jahr 2000 noch nicht ausreichende Töchterinformationen vorliegen (VIT, 2006b). Insgesamt liegt bei Holsteinbullen ein genetischer Trend in Höhe von etwa $111 \mathrm{~kg}$ Milch pro Jahr vor. Die mittleren Zuchtwerte Fettmenge steigern sich von ursprünglich durchschnittlichen -13,0 kg im Jahr 1988 auf 26,6 kg im Jahr 2001. Ein ähnliches Niveau erreicht auch die Eiweißmenge, die über die Jahre von -16,2 kg auf 30,2 kg zunimmt.

Auch die Zuchtwerte der Kühe deuten auf einen positiven Trend der Mengenmerkmale im Verlauf der letzten Jahre hin. Als Datengrundlage wurden die mittleren Zuchtwerte Schwarzbunter MLP-Kühe der Geburtsjahrgänge 1991 bis 2004 herangezogen (VIT, 2006b). Die Milchleistung entwickelt sich mit einer jährlichen durchschnittlichen Steigerung von etwa $99 \mathrm{~kg}$ deutlich positiv. Im jüngsten Geburtsjahrgang 2004 liegt der mittlere Zuchtwert für Milch auf einem Niveau von $428 \mathrm{~kg}$. Die Zuchtwerte für Fett- und Eiweißmenge verbessern sich vom ältesten untersuchten Jahrgang 1991 bis zum jüngsten Jahrgang 2004 um durchschnittlich 38,3 kg bzw. 40,4 kg pro Jahr. 
Annähernd identische Aufwärtsentwicklungen zeigen die Untersuchungen von Fürst (2006) mit Daten der Zuchtwertbank ZUCHTDATA in Österreich aus dem Jahr 2006. Fürst (2006) ermittelte bei der Rasse Holstein anhand mittlerer Bullenzuchtwerte der Geburtsjahrgänge 1990 bis 2000 eine durchschnittliche genetische Steigerung der Milchleistung von 114 kg pro Jahr. Der genetische Fortschritt bei Fleckvieh- und Braunviehbullen liegt mit durchschnittlich 97 kg bzw. 81 kg Milch pro Jahr deutlich unter dem Durchschnitt der Holsteinbullen. Zur Ermittlung der Milchleistungstrends bei Kühen, analysierte Fürst (2005) die Ergebnisse der Milchleistungskontrolle aller Kontrollkühe mit Vollabschlüssen. Die Untersuchung zeigt für den Betrachtungszeitraum 1995 bis 2004 eine Steigerung der durchschnittlichen Milchleistung von bis zu $200 \mathrm{~kg}$ Milch pro Kuh und Jahr bei stabilem Fett- und steigendem Eiweißgehalt (Fürst, 2005).

Im Zuge der weltweit zu verzeichnende Aufwärtsentwicklung der durchschnittlichen Milchleistung (Fürst 2005; 2006) berichten auch Swalve (1999) und Anacker (2003) von anhaltenden positiven genetischen Trends der Milchleistung in der deutschen Holsteinpopulation. Bezüglich der Inhaltsstoffe Fett- und Eiweiß verweist die Literatur auf negative Trends, wobei der Trend beim Fettgehalt deutlich ausgeprägter ist als beim Eiweißgehalt (Swalve, 1999; Anacker, 2003; Bergfeld, 2004).

Eine ausführliche Studie zu jährlichen genetischen Trends bei Schwarzbunten Kühen und Bullen in Deutschland wurde von Swalve und Höver (2003) durchgeführt. Für die Analyse standen innerhalb des Betrachtungszeitraums 1995 bis 2002 Zuchtwerte von rund 3 Mio. MLP-Kühen die sich in der ersten bis dritten Laktation befanden, aus den Zuchtgebieten Schleswig-Holstein (RSH), Niedersachsen (ZEH), Berlin-Brandenburg (RBB) und Mecklenburg-Vorpommern (RMV) zur Verfügung. Aus diesem Datenpool wurden zuerst 2.4 Mio. Kühe nach den Geburtsjahren 1992 bis 1999 herausgefiltert und anschließend die Kühe gewählt, deren Abstammung nachgewiesen werden konnte. Für die eigentliche Untersuchung des genetischen Fortschritts standen schliesslich knapp 2 Mio. Kühe mit entsprechenden mittleren Zuchtwerten der Jahrgänge 1992 bis 1999 zur Verfügung. Neben allgemein rückläufigen Trends der Milchinhaltsstoffe Fett- und Eiweiß-\%, zeigen Swalve und Höver (2003) jährliche genetische Fortschritte in Höhe von durchschnittlich $111 \mathrm{~kg}$ Milch, 3,2 kg Fett und 3,3 kg Eiweiß. Die Ergebnisse dieser Studie wurden mit denen der Holstein Population der USA und Kanada verglichen. Die aus den Daten des Animal Improvement Programs Laboratory (AIPL) für den gleichen Betrachtungszeitraum, aber bei kleinerer Stichprobengröße pro Geburtsjahrgang, berechneten genetischen Trends liegen bei $109 \mathrm{~kg}$ Milch pro Jahr und 3,3 kg bzw. 3,4 kg für Fett- und Eiweißmenge. Die vom Canadian Dairy Network (CDN) bereitgestellten Daten zur Ermittlung der genetischen 
Trends, beziehen sich nur auf einen Zeitraum von 1992 bis 1997. Abgesehen von einer noch kleineren Stichprobengröße als bei der amerikanischen Untersuchung, liegen die genetischen Fortschritte bei $172 \mathrm{~kg}$ Milch, 4,7 kg Fett und 5,4 kg Eiweiß pro Jahr (Swalve und Höver, 2003).

In einer weiteren amerikanischen Studie von van Tassel und van Vleck (1991) standen von 6000 Holstein-Bullen und 1 Mio. Holstein-Kühen des Geburtsjahrgangs 1981 und jünger Datensätze aus der ersten Laktation zur Verfügung. Anhand der mittleren Zuchtwerte wurde der genetische Trend der Milchleistung pro Geburtsjahrgang in vier Selektionspfaden ermittelt. Insgesamt zeigen die Ergebnisse von van Tassel und van Vleck (1991) bei den Kühen einen genetischen Zuchtfortschritt in Höhe von $21 \mathrm{~kg}$ bis $51 \mathrm{~kg}$ Milch pro Jahr. Bei den Bullen variierte der Trend von $36 \mathrm{~kg}$ bis $98 \mathrm{~kg}$ Milch pro Jahr. Auch Abdallah und McDaniel (2000) erhoben anhand von 8.575 Holstein-Kühen mit mehr als 23.000 Datensätzen der ersten bis sechsten Laktation genetische Trends für die Geburtsjahrgänge 1950 bis 1993. Der genetische Trend der Leistung pro Jahr zeigt sich erst ab dem Geburtsjahrgang 1980 mit 94,7 kg Milch deutlich positiv. Auch für die Fettmenge zeigt die Untersuchung von Abdallah und McDaniel (2000) im Verlauf der Jahre insgesamt eine positive Entwicklung. Die deutlichste ist auch hier erst zwischen den Jahrgängen 1980 bis 1993 mit durchschnittlich 3,46 kg Fett pro Jahr zu verzeichnen.

Die Verbesserung im Management und auch züchterische Maßnahmen sind nach Fürst (2006) Ursachen der weltweit zu beobachtenden Aufwärtsentwicklung der durchschnittlichen Milchleistung. Als Beleg dafür zeigt die Arbeit von Dunklee et al. (1994), dass durch gezielte Anpaarungen von Kühen mit Bullen, die hohe Zuchtwerte besitzen, auch deutlich positive Trends in der Milchleistung erreicht werden. Im Rahmen eines Zuchtversuches wurden 2.768 Laktationsdaten von 1.078 Kühen erfasst und die Versuchsherden in zwei genetisch unterschiedliche Gruppen eingeteilt. In einer Gruppe wurde nur Sperma von Bullen mit hohen Zuchtwerten eingesetzt, in der Vergleichsgruppe Sperma von Bullen mit durchschnittlichen Zuchtwerten. Die Ergebnisse zeigen, dass die genetisch besser veranlagte Gruppe $940 \mathrm{~kg}$ Milch bzw. 16,4 \% mehr Milch pro Laktation erzielen als die Vergleichsherde. Der genetische Trend liegt entsprechend bei $70 \mathrm{~kg}$ Milch pro Jahr. Auch die Lebensleistung erhöht sich um 15,7 \% bzw. 3.435 kg Milch. Fett- und Eiweißmenge steigen ebenso um 8,0 bzw. 12,4 \% an (Dunklee et al., 1994). 
Annähernd gleiche Leistungssteigerungen illustrieren die Ergebnisse einer skandinavischen Studie von Bo (2004). Die Zuchtwerte dänischer Bullen, Väter und Muttersväter der Rasse Holstein-Friesian, zeigen über die Jahre 1984 bis 2000 eine konstante positive Entwicklung für den Relativzuchtwert Milchleistung. Insgesamt zeigt sich für dieses Merkmal und für den gesamten Betrachtungszeitraum eine Steigerung von mehr als $20 \%$ (Bo, 2004).

Eine Übersicht phänotypischer Trends von Leistungs- und funktionalen Merkmalen der Rasse Holstein-Friesian auf ökologischen Milchviehbetrieben in den Niederlanden, zeigt die Untersuchung von Nauta et al. (2006a). Aus 46.282 Daten erstlaktierender Kühe ökologischer Betriebe und 184.282 Kuhdaten konventioneller Betriebe, wurden für die Geburtsjahrgänge 1990 bis 2003 die phänotypischen Trends der Merkmale Milch-kg, Fettund Eiweiß-\%, Zellzahl, Zwischenkalbezeit und Erstkalbealter erhoben. Das Erstkalbealter der ausgewählten Kühe betrug zwischen 17 und 36 Monaten, die Rastzeit zwischen 30 und 250 Tagen. Alle benötigten Daten wurden vom niederländischen Herdbuch und der niederländischen Organisation für Milchleistungsprüfung zur Verfügung gestellt. Die Daten wurden betriebsspezifisch nach ökologischen -, Umstellungs- und konventionellen Betrieben geordnet und miteinander verglichen.

Der Vergleich ökologischer mit konventionellen Betrieben, zeigt in Hinsicht auf die Milchleistung erstlaktierender Kühe, dass ökologische Betriebe im gesamten Betrachtungszeitraum mit etwa $830 \mathrm{~kg}$ Milch unter dem Leistungsniveau der konventionellen Vergleichsbetriebe liegen. Auch die phänotypischen Trends für den Fett- und Eiweißgehalt liegen mit $0,12 \%$ und $0,08 \%$ unter den konventionellen Werten. Insgesamt betrachtet zeigt sich für den Fettgehalt auf beiden Betriebsformen eine gleiche rückläufige Entwicklung von 4,5 \% im Jahr 1990 auf 4,3 \% im Jahr 2003 (Nauta et al., 2006a). In einer weiteren Untersuchung zeigen Rozzi et al. (2007) annähernd ähnliche Entwicklungen für Leistungs- und einige funktionale Merkmale bei Holsteinkühen auf ökologischen Betrieben in Kanada. Anhand der Milchleistungsdaten konnte für die Jahre 1998 bis 2003 eine durchschnittliche Milchleistung der Ökokühe von 8.069 kg, mit 3,93 \% bzw. $317 \mathrm{~kg}$ Fett und 3,23 \% bzw. $260 \mathrm{~kg}$ Eiweiß festgestellt werden. Diese Milchleistung liegt jedoch um $19 \%$ unter der konventionellen Leistung. Im Gegensatz dazu sind aber Fett- und Eiweiß-\% um 0,28\% bzw. 0,04\% höher als die in denselben Jahren erbrachte Leistung auf konventionellen Betrieben. Nauta et al. (2006a) beschreiben auch für das Merkmal Zellzahl eine negative Entwicklung auf ökologischen Betrieben. Die Werte liegen im Mittel deutlich höher als auf konventionellen Betrieben. Rozzi et al. (2007) fanden ähnliches heraus. Beide wissenschaftlichen Studien zeigen, dass die Zellzahl auf ökologischen Betrieben im Schnitt um 
50.000 Zellen pro ml Milch höher liegt als in konventionellen Milchviehbetrieben. Auch für die Merkmale Zwischenkalbezeit und Erstkalbealter zeigen Nauta et al. (2006a) höhere Werte auf ökologischen Milchvieh-betrieben. Bei der Zwischenkalbezeit kann für die letzten Jahre ein Anstieg von durchschnittlich 23 Tagen beobachtet werden. Im konventionellen Bereich zeigt sich ein Anstieg um nur etwa 12 Tage. Auch das Alter der Kuh bei Geburt des ersten Kalbes ist auf ökologischen Betrieben in den letzten Jahren mit durchschnittlich 27 Monaten im Mittel einen Monat höher als auf konventionellen Betrieben. Bei noch nicht lange bestehenden Ökobetrieben zeigt sich nach der Umstellung von der konventionellen Wirtschaftsform auf die ökologische eine Zunahme des Erstkalbealters um 1,3 Monaten innerhalb von sieben Jahren.

Die Entwicklung der Zwischenkalbezeit auf ökologischen Betrieben ist ebenso ein Teil der Untersuchung von Reksen et al. (1999). Sie verglichen in ihrer Studie 998 Laktationsdaten ökologischer und 3.016 Laktationsdaten konventioneller Milchviehbetriebe in Norwegen. Anders als bei den bisher erwähnten Studien, beziehen sich die Ergebnisse auf die Rasse Norwegisches Rotvieh, das in dieser Untersuchung zu $85 \%$ auf den ökologischen Betrieben und zu $97 \%$ auf den konventionellen Betrieben gehalten wurde. Innerhalb des Betrachtungszeitraums von 1994 bis 1996 stellen Reksen et al. (1999) durchschnittliche Zwischenkalbezeiten von 378,4, 376,4 und 369 Tagen bei den Ökokühen fest. Konventionelle Kühe liegen im Vergleich dazu bei durchschnittlich 377,8, 375,1 und 374,1 Tagen, was aber keinen signifikanten Unterschied darstellt. Ein allgemeingültiges Fazit konnten Reksen et al. (1999) nach Verwendung eines gemischten Modells, bei dem alle Störfaktoren eliminiert wurden, ziehen. Konventionelle Betriebe zeigen demnach mit 374,1 Tagen insgesamt eine kürzere Zwischenkalbezeit als Ökobetriebe mit 382,7 Tagen.

\subsection{Die Entstehung des ökologischen Landbaus - ein historischer Rück- blick}

Ausgelöst durch eine Landwirtschaftskrise, die zu einem Ertragseinbruch und sinkender Nahrungsmittelqualität führte, wurde bereits zwischen den beiden Weltkriegen der Grundstein der heutigen ökologischen Wirtschaftsweise gelegt. Nach Vogt (2001a) wird die geschichtliche Entwicklung des Ökolandbaus durch fünf ökologische Landbausysteme beschrieben.

Die bis in den Jahren 1920 und 1930 betriebene chemisch-technische Intensivierung unterlag durch die Entdeckung stickstoffbindender Bakterien durch Hermann Hellriegel und Hermann Wilfahrt einem biologisch orientierten Wandel. Das 1911 in diesem Zusammen- 
hang erschienene „Handbuch der Landwirtschaftlichen Bakteriologie“, von Felix Löhnis (Vogt, 2001a), diente als Leitfaden zur Intensivierung der Landbewirtschaftung über eine verbesserte Humuswirtschaft. Ende des 19. Jahrhunderts verfolgte die LebensreformBewegung das Ziel der Rückkehr einer naturgemäßen Lebensweise. Dies wurde einerseits durch den Verzicht auf den Einsatz stickstoffhaltiger Mineraldünger und schwermetallhaltiger Pestizide, aber auch aufgrund vegetarischer Grundsätze durch den Verzicht der Tierhaltung zu Nahrungszwecken umgesetzt. In diesem vieharmen Landbau indem die wenigen Tiere lediglich als Arbeitskräfte und zur Milch- und Wollerzeugung gehalten wurden, finden sich nach Vogt (2001a) erste Ansätze artgemäßer Tierhaltung wieder. Aus diesem Prozess entwickelte sich die Landwirtschaftliche Bakteriologie. Unterstützt durch Ewald Könemanns dreiteiliges Werk „Biologische Bodenkultur und Düngewirtschaft“, welches die Konzepte des Natürlichen Landbaus zusammenfasst (Vogt, 2001a), wurde die Nachhaltigkeit der Landbewirtschaftung gesteigert. Erste Richtlinien, Gütesiegel und lokale Vermarktungsprojekte wurden durch die 1927/28 gegründete Organisation Arbeitsgemeinschaft Natürlicher Landbau und Siedlung (ANLS), 1935 umbenannt in Arbeitsgemeinschaft Landreform, initiiert (Vogt, 2001a).

Im Jahr 1924 entstand neben dem Natürlichen Landbau ein zweites ökologisches Landbausystem, die Biologisch-Dynamische Wirtschaftsweise. Begründer dieser Landbewirtschaftung und gleichzeitig Begründer der Anthroposophie war Rudolf Steiner (Vogt, 2001a). Durch dieses biologisch-dynamische Konzept wurde nach Vogt (2001a) der Betrieb als eine eigenständige, lebendige Wesenheit aufgefasst. Schließlich wurde um 1930 das Gütesiegel Demeter eingeführt. In den darauf folgenden Jahren 1950 und 1960 wurden die biologisch-dynamischen Konzepte durch Integration wissenschaftlicher Erkenntnisse an den naturwissenschaftlich orientierten Landbau herangeführt. Begründer des organisch-biologischen Landbaus war der Schweizer Agrarpolitiker Hans Müller (Vogt, 2001b). Mit dieser Wirtschaftsweise, wozu der Frankfurter Arzt und Mikrobiologen Hans Peter Rusch den theoretischen Hintergrund lieferte, wurde ab dem Jahr 1950 das Ziel verfolgt, hochwertige Nahrungsmittel zu erzeugen und diese zu gerechten Preisen an gesundheitsbewusste Verbraucher abzugeben. Zudem sollte dadurch die Unabhängigkeit der Landwirte gegenüber der Landwirtschafts- und Ernährungsindustrie gewährleistet werden. Zum Ende des Jahres 1970 und zum Anfang des Jahres 1980 erfolgte eine erste wissenschaftliche Anerkennung der ökologischen Landbewirtschaftung (Vogt, 2001b). Im Jahr 1988 schlossen sich die Anbauverbände in Deutschland zu einem Dachverband, der Arbeitsgemeinschaft ökologischer Landbau (AGÖL), zusammen. Seit 1980 und 1990, stehen schließlich eine umweltschonende Bodenbewirtschaftung und eine artgerechte Tierhaltung im Vordergrund. 


\subsubsection{Zahlen des ökologischen Landbaus und der Entwicklung der ökologischen Milchviehhaltung in Deutschland}

In den vergangenen Jahren ist für den ökologischen Landbau in Deutschland ein kontinuierliches Wachstum zu verzeichnen. So wurden am 31.12.2004 von insgesamt 16.603 Betrieben 767.891 ha Land nach der EG-Verordnung Nr. 2092/91 ökologisch bewirtschaftet, was 3,9\% der landwirtschaftlichen Betriebe insgesamt und 4,5\% der gesamten landwirtschaftlich genutzten Fläche in Deutschland entspricht. Zum Ende des Jahres 2005 wirtschafteten bereits 17.020 landwirtschaftliche Betriebe auf 807.405 ha Fläche nach ökologischen Bestimmungen. Der Anteil ökologischer Betriebe ist somit auf 4,2\% der gesamten Anzahl der Betriebe und die ökologische Nutzfläche auf 4,7\% der insgesamt bewirtschafteten Fläche in Deutschland gestiegen (BMELV, 2007).

Im Jahr 2006 gehörten 9.645 Betriebe, 0,4 \% bzw. 40 Betriebe mehr als im Vorjahr, einem ökologischen Anbauverband an. Unter Organisation dieser Verbände wurden nach Angaben des Bund Ökologischer Lebensmittelwirtschaft (BÖLW) 562.792 ha landwirtschaftliche Fläche bewirtschaftet (Dosch und Gerber, 2007). Dies entspricht im Vergleich zum Vorjahr einer Zunahme um 2,8 \% bzw. 15.193 ha mehr ökologisch bewirtschafteter landwirtschaftlicher Fläche.

Auch die ökologische Milchproduktion unterliegt einem kontinuierlichen Wachstum. Nach Angaben des Statistischen Bundesamt Deutschland (DESTATIS) ist die Zahl der Milchkühe auf ökologisch bewirtschafteten Betrieben von 85.300 Kühen im Jahr 1999 über 102.500 Kühe im Jahr 2001 auf 109.600 Kühe im Jahr 2003 gestiegen (DESTATIS, 2005). Abbildung 3 zeigt anhand der geographischen Darstellung der Bundesrepublik Deutschland, die Verteilung ökologischer Milchviehbetriebe je $\mathrm{km}^{2}$ landwirtschaftlicher Fläche in den Landkreisen (Nieberg und Deeken, 2005). Die dunkel eingefärbten Gebiete zeigen die Ballungsräume ökologischer Milchviehbetriebe an. Es ist zu erkennen, dass vor allem im süddeutschen Raum Milchviehhaltung nach ökologischen Richtlinien betrieben wird. 


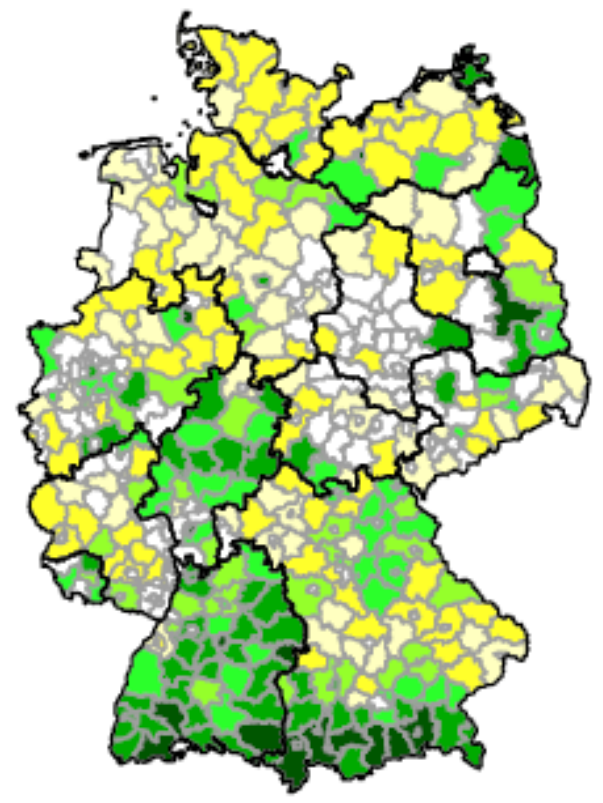

Öko-Kühe je $\mathrm{km}^{2} \mathrm{LF}$ in den jeweiligen Landkreisen

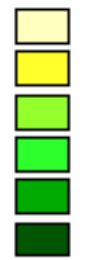

$<=0,1$

$0,1<=0,3$

$0,3<=0,5$

$0,5<=1,0$

$1,0<=3,0$

$>3,0$

Abbildung 3: Regionale Verteilung der Ökokühe in Deutschland (Nieberg und Deeken, 2005).

Der Anteil der nach den Richtlinien des ökologischen Landbaus erzeugten Milch lag im Jahr 2002 mit 325 Mio. kg produzierter Milch bei etwa 1,2 \% an der gesamten deutschen Milcherzeugung (Schumacher, 2002). Im Jahr 2003 lag der Anteil schon bei 1,3 \% (Hollenberg-Koch und Schoch, 2004) und im Jahr 2004 mit ca. $430.000 \mathrm{t}$ Milch bei 1,5\% an der gesamten Milchproduktion in Deutschland (Rippin, 2006). Bei der Milchanlieferung an die Molkereien gab es im Jahr 2003 im Vergleich zum Vorjahr einen Zuwachs von 8,6 \% (Hollenberg-Koch und Schoch, 2004; Barth und Rahmann, 2005). Das von Redelberger et al. (2002) und Schumacher (2002) prognostizierte Wachstum der Milchmenge von $10 \%$ bzw. 20 bis $35 \%$, kann durch aktuellere Studien bislang nicht bestätigt werden. Ausgelöst durch tendenziell rückläufige Bio-Erzeugerpreise seit dem Preishoch im Jahr 2001, zeigen die Analysen von Rippin (2006), dass die Erzeugung von ökologischer Milch im Jahr 2005 trotz Umsatzzuwächsen von 20 \% nur um $3 \%$ gestiegen ist. Aufgrund der rückläufigen Basispreise für Biomilch und der gleichzeitigen Angebotsverknappung bleibt abzuwarten, wie sich der Markt zukünftig entwickelt (Rippin, 2006).

Die heutige Jahresmilchleistung pro Kuh ist im Ökolandbau von einem früheren Niveau zwischen 3.000 und 5.000 I Milch auf ein Niveau zwischen 5.000 und 9.000 I Milch pro Jahr gestiegen (Schumacher, 2004). Diese Leistung liegt im Durchschnitt 10 bis $15 \%$ unter dem konventionellen Durchschnitt. Das niedrigere Leistungsniveau wird durch einen höheren Milchpreis ausgeglichen, der sich im Mittel inklusive aller Zu- und Abschläge um ca. 5 ct über dem konventionellen Milchpreis bewegt (Rippin, 2006). 


\subsubsection{Richtlinien der ökologischen Milchviehhaltung}

Die ökologische Milchviehhaltung in Deutschland unterliegt einer dreistufigen Hierarchie. Demnach muss neben verbandspezifischen Bedingungen ein in Europa verbreiteter einheitlicher Mindeststandard für die ökologische Tierhaltung eingehalten werden, der sich an weltweiten Bestimmungen der IFOAM (International Federation of Organic Agriculture Movement) orientiert. Die IFOAM agiert als Dachverband der biologischen Landbaubewegung, und ihre Basisrichtlinien dienen als Grundlage aller neu zu definierenden Verordnungen, je nach lokalen, sozioökonomischen, geoklimatischen und kulturellen Bedingungen.

Als Orientierung für nachfolgende Entwicklungen neuer bzw. ergänzender Gesetzgebungen im ökologischen Landbau, dient der seit dem Jahr 1999 bestehende Gremium Codex Alimentarius der FAO (Food and Agriculture Organisation of the United Nations) (SOEL, 2006). In diesem Codex ist der Ökologische Landbau eindeutig definiert und beschreibt diese Wirtschaftsweise als ein auf Nachhaltigkeit ausgerichtetes holistisches Produktionssystem für Pflanzen und Tiere, welches den innerbetrieblichen Nährstoffkreislauf unterstützt und sich gegen den Einsatz von chemisch-synthetischen Hilfsstoffen richtet (FAO, 2001).

Die Umsetzung gemeinschaftlicher Rahmenvorschriften über Erzeugung, Etikettierung und Kontrolle zur gesetzlichen Absicherung ökologisch erzeugter Lebensmittel auf europäischer Ebene ist seit 1991 durch die EU-Öko- Verordnung (EWG) Nr. 2092/91 geregelt (SOEL, 2006). Am 19. Juli 1999 wurde, ergänzend zum bereits geregelten Pflanzenbau, die durch den Agrarrat beschlossene Verordnung EG Nr. 1804/1999 zur Tierhaltung in die Verordnung des ökologischen Landbaus mit einbezogen. Seit dem 24. August 2000 gilt somit auch ein einheitlicher Mindeststandard für die ökologische Tierhaltung. Nach dieser Verordnung müssen detaillierte Bestimmungen im Bereich der Zucht, Haltung, Fütterung und Krankheitsvorsorge sowie zum Tierbesatz beachtet werden (SOEL, 2006).

Die meisten Betriebe mit dem Schwerpunkt ökologische Milchviehhaltung sind in einem Verband organisiert und wirtschaften nach verbandsspezifischen Richtlinien. Einige dieser Richtlinien sind in einzelnen Punkten strenger als die EG-Öko-Verordnung, da ein Betrieb nach diesen Prinzipien auch nur teilweise umgestellt werden kann. Die Verbände fordern dagegen immer eine gesamte Umstellung des Betriebes als Voraussetzung zur Förderung in Deutschland (BMELV, 2007). Die Verbände Biokreis e.V. (1979), Bioland e.V. (1971), Demeter-Bund (1924), Gäa-Vereinigung ökologischer Landbau (1989) und Naturland (1982) sind die sechs von insgesamt acht Bioverbänden in Deutschland, die nach 
verbandsspezifischen Vorgaben auch den Sektor Milchviehhaltung betreuen. Mit mehr als 4.500 Mitgliedern, die mehr als 200.000 ha landwirtschaftliche Fläche bewirtschaften, ist BIOLAND e.V. der größte Anbauverband in Deutschland (Dosch und Gerber, 2007).

\section{4 Ökologisches Zuchtziel}

Oberstes Ziel der ökologischen Milchviehhaltung ist es, „mit gesunden, langlebigen und rasse- und artspezifisch leistungsstarken Tieren eine ökologische und ökonomische Produktion hochwertiger Lebensmittel zu erreichen“ (MUNLV.NRW, 2002). Als Maßstab zur Erreichung des Zuchtziels dienen nach Postler und Schmidt (2003) und Postler (2005; 2006)

- eine angepasste Lebensleistung und eine lange Nutzungsdauer,

- gute Fitness und Vitalität,

- eine hohe Grundfutterleistung,

- eine hohe Persistenz in der Milchleistung,

- ein gesundes Euter und eine gute Melkbarkeit,

- gesunde Klauen und ein gutes Fundament und

- eine gute Fruchtbarkeit.

Eine Herde mit einer hohen durchschnittlichen Nutzungsdauer und einer guten Tiergesundheit wirkt sich nach Postler (2002a) positiv auf die Remontierungs-, Tierarzt-, Futterund Stallbaukosten aus, was sich wiederum positiv auf die Wirtschaftlichkeit eines Betriebes auswirkt.

\subsubsection{Zuchtorganisationen und Zuchtprogramme der ökologischen Milchviehhal- tung}

Nach Postler (2006) stellt die Lebensleistung einer Kuh das Fundament jeglicher Zuchtarbeit dar. Anhand dieses Merkmals können weitere wichtige Kriterien wie Gesundheit, Fruchtbarkeit und die Milchleistung in ihrer Ausprägung und Kombination in einer für das Tier optimalen Art und Weise erfasst werden (Postler, 2002a; 2006). Weitere Vorteile einer hohen Nutzungsdauer und Lebensleistung sieht Postler (2002b; 2006) darin, dass mehr Kühe durch eine natürliche Leistungssteigerung, bedingt durch den Reifungsprozess des Organismus bis etwa zur vierten Laktation, ein Leistungsmaximum erreichen. Auch zu diesem Zeitpunkt sind die Organe soweit angepasst, dass durch ein höheres Futteraufnahmevermögen eine höhere Grundfutterleistung erzielt werden kann. Neben der Erhöhung der Selektionsintensität, vor allem auf der weiblichen Seite, steigt auch der 
Unternehmergewinn durch steigende Deckungsbeiträge pro Kuh bis zur neunten Laktation an. Außerdem bleiben den Tieren aufgrund geringer Remontierung Unruhen und Stress durch Wechsel im Bestand erspart, dessen Herdenstruktur sich schließlich aus 1/3 jungen, 1/3 mittleren und 1/3 älteren Tieren zusammensetzen würde (Postler, 2002b).

Mit der Durchführung eines Linienzuchtprogramms kann nach Postler (2002a) sichergestellt werden, dass eine hohe Lebensleistung weitervererbt und innerhalb einer Familie fest verankert wird. Für die Weitergabe guter Konstitutions- und Leistungseigenschaften an die nächste Generation, müssen beide Elternteile aus einer Familie oder Zuchtlinie stammen, die durch Erreichen einer hohen Lebensleistung gekennzeichnet ist. Sind mehrere Familien oder Zuchtlinien nicht miteinander verwandt, müssen diese im Rahmen des Zuchtprogramms abwechselnd angepaart werden, um die Inzuchtrate so gering wie möglich zu halten. Außerdem soll durch diese so genannte Rotationsanpaarung (Abbildung 4) erreicht werden, dass sich die Vererbungssicherheit durch die Anhäufung der Lebensleistungsmerkmale, wie Dauerleistung und Langlebigkeit, verbessert (Postler, 2002b).

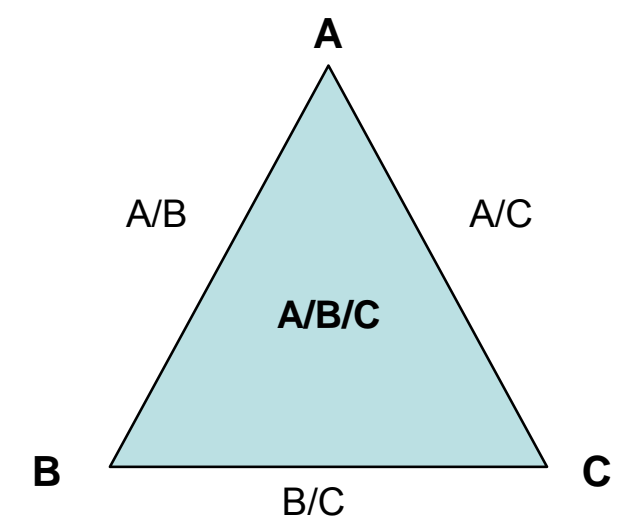

Abbildung 4: Darstellung des Rotationsanpaarungsmodells (Postler, 2002b).

Diese Zuchtprogramme basieren auf den im Jahr 1958 von Professor Frederik Bakels durchgeführten Arbeiten zur Lebensleistungszucht (Postler, 2002b), die die Forschung in der ökologischen Rinderzucht nachhaltig geprägt haben. International gibt es derzeit fünf Vereine bzw. Lebensleistungsorganisationen, die ein auf Lebensleistung ausgerichtetes Zuchtprogramm verfolgen. Unter Abstimmung aller Lebensleistungsorganisationen wird jährlich ein LL-Bullenkatalog zur Rinderzucht auf Lebensleistung herausgegeben. 
Im Einzelnen handelt es sich dabei um die

- ARGE - Arbeitsgemeinschaft für Rinderzucht auf Lebensleistung, unter der Leitung von Dr. Günter Postler

- $\mathrm{ALL}$ - Arbeitsgemeinschaft Lebenslinien, unter der Leitung von Dr. Karl Wittenberg

- AÖLZ - Arbeitsgemeinschaft Österreichischer Lebensleistungszüchter, unter der Leitung von Martin Ertl

- Verein zur Erhaltung und Förderung des alten schwarzbunten Niederungsrindes e.V., unter der Leitung von Hans-Jürgen Euler und den

- Arbeitskreis Holländischer Lebensleistungszüchter, unter der Leitung von Wytze Nauta.

Die ARGE wurde im Jahr 1983 als eine Initiative von Rinderzüchtern für Züchter gegründet und dient als europäische Koordinationsstelle für die Zucht auf Lebensleistung (Postler, 2005). Das Zuchtziel der Arbeitsgemeinschaft für Rinderzucht auf Lebensleistung ist unabhängig von der Rasse und der Nutzungsrichtung und verfolgt „die Zucht problemloser, dauerleistungsveranlagter Rinder mit hoher Nutzungsdauer und Lebensleistung, unter Beachtung der Naturgesetze von Körperbau und Leistung“ (Postler, 2005). Durch eine hohe Dauerleistungsbereitschaft, eine hohe Anpassungsfähigkeit und einer hohen Selektionsintensität sollen Ökologie und Ökonomie miteinander verknüpft werden. Die wesentlichen Arbeitsaufgaben dieser Lebensleistungsorganisation liegen nach Postler (2005) in der Beratung bei Zucht-, Haltungs-, Fütterungs-, Gesundheits- und Managementfragen, sowie in der Zuchttier- und Spermavermittlung. Ein weiteres Anliegen der ARGE ist die Förderung des Erfahrungsaustausches. Dafür werden Fachtagungen, Seminare und Exkursion durchgeführt und ergänzend dazu Rundbriefe, Informationsbroschüren und Fachartikel herausgegeben.

Die ALL, Arbeitsgemeinschaft Lebenslinien, wurde 1988 durch Dr. Karl Wittenberg gegründet. In Anlehnung an die ARGE verfolgt die ALL das Ziel, eine Kuh zu züchten, die unter tiergerechten hiesigen Bedingungen für den bäuerlichen Betrieb die beste Wirtschaftlichkeit in der Erzeugung von genusstauglicher Milch und vitaler Nachkommen erzielt (Postler und Schmidt, 2003). Als Maßstab zur Überprüfung des Zuchtziels, dienen eine hohe Lebensleistung und Nutzungsdauer, eine gute körperliche Konstitution und funktionelle Fitness, eine ungestörte Fruchtbarkeit, ein gesundes gut melkbares Euter, eine konstante Persistenz während der Laktation und eine sichtbare Leistungssteigerung von Laktation zu Laktation. Da die ALL bei der Anwendung der Linienzucht großen Wert auf bewährte Kuhfamilien legt, werden die gewünschten Eigenschaften bei der Auswahl der Bullenväter, der Bullenmütter und der für den Prüfeinsatz vorgesehenen Jungbullen 
berücksichtigt und in den seit 1996 im Rechenzentrum Verden erarbeiteten Gesamtzuchtwert-Lebensleistung (GZ-LL) mit einbezogen. Dieser Gesamtzuchtwert gewichtet die Einzelmerkmale nach ihrer züchterischen und betriebswirtschaftlichen Bedeutung im nachhaltigen Sinne. Die höchste Einflussgröße auf den GZ-LL ist der Relativzuchtwert Nutzungsdauer mit einer Gewichtung von 28 \% (Postler und Schmidt, 2003; Postler, 2005). Wesentliche Zuchtmethoden der ALL sind die Familien-/Basiszucht, die Rotationszucht und die Kombinationszucht.

Bei der AÖLZ werden nach Haiger (2006) folgende Kriterien zur Auswahl eines Bullen festgelegt: Familien mit hohen Lebensleistungen, Fitness (Nutzungsdauer, Persistenz und Zellzahl) und ZW-Milch (Fett- und Eiweißmenge). Der Fleischwert ist in diesem Zuchtprogramm unbedeutend. Kuhfamilien, in denen hohe Lebensleistungen gehäuft vorkommen, sind demnach das erste und wichtigste Auswahlkriterium. Im zweiten Schritt werden die Bullen anhand ihrer Zuchtwertschätzung durch Töchter mit drei oder mehr abgeschlossenen Laktationen nach den Fitnesseigenschaften rangiert. Anschließend werden diese Bullen nach ihrem Milch-Zuchtwert geordnet (Ertl und Haiger, 2005; Haiger, 2006).

Der Verein zur Erhaltung und Förderung des alten schwarzbunten Niederungsrindes wurde 1989 gegründet. Diese Zuchtvereinigung verfolgt als erste Priorität, HF-freie Schwarzbunte als Zweinutzungsrind mit einem Milch - Fleischverhältnis von 60:40 zu erhalten und diese Rasse vor dem Aussterben zu bewahren. Charakteristische Merkmale dieser Rasse sind gute Fundamente, eine hohe Widerstands- und Anpassungsfähigkeit auch bei unterschiedlichen Klimaverhältnissen, die Eignung zur Landschaftspflege und gute Milchleistungs- und Masteigenschaften auf ökologischen und konventionellen Betrieben. Das Ziel des Vereins ist die Zucht einer langlebigen Dauerleistungskuh mit viel Körpersubstanz (Postler und Schmidt, 2003; Postler, 2005).

\subsection{2 ÖZW - Ökologischer Gesamtzuchtwert}

Nach Krogmeier et al. (2007) ist der Ökologische Gesamtzuchtwert (ÖZW) ein zusammenfassender Wert über alle vorliegenden Abstammungs- und Leistungsdaten der Besamungsbullen, der den Zielsetzungen und Rahmenbedingungen des ökologischen Landbaus in besonderer Weise Rechnung trägt. Der ÖZW ist aus einem anwendungsorientierten Forschungsprojekt „Ökologische Tierzucht und Tierhaltung“ entstanden, welches in den Jahren 1995 bis 1998 an der Bayerischen Landesanstalt für Tierzucht unter der Leitung von Dr. Günter Postler durchgeführt wurde (Postler, 2006). An diesem Projekt mitbeteiligt waren die bayerischen Ökoverbände Bioland, Naturland, Demeter und der 
Biokreis Ostbayern. Eine ÖZW-Liste erscheint zweimal pro Jahr und bietet den Landwirten Informationen und Entscheidungshilfen für ihre Zuchtarbeit (Postler, 2006). Nur Bullen, bei denen die Verbleiberate der Töchter nach 48 Monaten vorliegt, werden in die Liste aufgenommen. Je Besamungsbulle müssen außerdem von mindestens 20 Töchtern mindestens 3 Probemelkergebnisse der 3. Laktation vorliegen, damit dieser nach ÖZW rangiert werden kann. Die Berechnung des Ökologische Gesamtzuchtwertes erfolgt seit 2005 mittels der Selektionsindexmethode. Somit können Erblichkeitsgrade, genetische Korrelationen, Sicherheiten der geschätzten Zuchtwerte und wirtschaftliche Gewichte der Merkmale mit berücksichtigt werden. Einzelne Teilwerte werden je nach Bedeutung für die Leistungsfähigkeit, unter besonderer Berücksichtigung der Lebensleistung und Nutzungsdauer, Tiergesundheit und Langlebigkeit unterschiedlich gewichtet. Infolgedessen wird im ÖZW der Teilwert Leistung zu $35 \%$ und der Teilwert Konstitution zu 65 \% gewichtet. Innerhalb der Teilwerte erfolgen weitere Unterteilungen in die Teilzuchtwerte (Krogmeier et al., 2007).

Der Teilwert Leistung ist in den Ökologischen Milchwert (ÖMW) und den Fleischwert (FW) unterteilt. Im ÖMW werden die Zuchtwerte Fett- und Eiweiß-kg je Laktation in einem Verhältnis von 1:4 gewichtet. Die Laktationen werden dabei unterschiedlich gewichtet. Daraus ergibt sich ein Verhältnis zwischen der 1., 2. und weiterer Laktationen von 10:20:70. Der Fleischwert wird unverändert aus der konventionellen Zuchtwertschätzung übernommen (Postler, 2006; Krogmeier et al., 2007). Der Teilwert Konstitution ist unterteilt nach den Teilzuchtwerten Persistenz und Leistungssteigerung (PL), Nutzungsdauer (ND), Kalbung und Vitalität (KV) und nach Form und Euter (FE). Im Teilzuchtwert PL wird der Zuchtwert Persistenz zu 40 \% und der Zuchtwert Leistungssteigerung zu $60 \%$ gewichtet. Die Nutzungsdauer wird als Merkmal für die Langlebigkeit ermittelt und dient als Maßstab für Lebensleistungsveranlagungen. Da es sich um eine funktionale bzw. leistungsunabhängige Nutzungsdauer handelt, kann der Effekt der leistungsbedingten Merzung im Rahmen der Zuchtwertschätzung ausgeschaltet werden (Krogmeier et al., 2007). Innerhalb des Teilzuchtwertes Kalbung und Vitalität kommt den maternalen Zuchtwerten die größte Bedeutung zu. Die maternale Fruchtbarkeit erhält demnach eine Gewichtung von $30 \%$, das Merkmal Kalbeverlauf maternal $25 \%$, die Totgeburtenrate maternal ebenfalls $25 \%$ und die paternalen Merkmale Kalbeverlauf und Totgeburtenrate jeweils nur $10 \%$. Die Zuchtwerte des Teilwertes Form und Euter sind bedeutend für ein gutes Fundament und ein funktionales und gesundes Euter. Demzufolge werden $30 \%$ dem Zuchtwert Euter, $20 \%$ dem Zuchtwert Form / Fundament, 10 \% Zuchtwert Trachten und jeweils $20 \%$ dem Zuchtwert Zellzahl und dem Zuchtwert Melkbarkeit zugeteilt. 
Aus den Berechnungen nach Krogmeier et al. (2007) und der Neugewichtung der Zuchtwertteile im ÖZW geht hervor, dass der zukünftige Zuchtfortschritt in etwa zu $50 \%$ im Leistungsbereich und zu 50 \% im Bereich der Konstitution liegt. Die monetären Zuchtfortschritte der einzelnen Merkmale sind in Tabelle 1 aufgelistet.

Tabelle 1: Der Anteil der einzelnen Merkmalsbereiche am monetären Zuchtfortschritt (Krogmeier et al., 2007).

\begin{tabular}{lccc}
\hline & Fleckvieh & Braunvieh & Gelbvieh \\
\hline Teilwert Leistung & $48,0 \%$ & $50,1 \%$ & $48,0 \%$ \\
Ökologischer Milchwert & $35,8 \%$ & $47,9 \%$ & $35,8 \%$ \\
Fleischwert & $12,2 \%$ & $2,2 \%$ & $12,2 \%$ \\
Teilwert Konstitution & $52,0 \%$ & $49,9 \%$ & $52,0 \%$ \\
Persistenz und Leistungssteigerung & $11,4 \%$ & $12,1 \%$ & $11,4 \%$ \\
Nutzungsdauer & $11,8 \%$ & $12,5 \%$ & $11,8 \%$ \\
Kalbung und Fruchtbarkeit & $17,1 \%$ & $14,7 \%$ & $17,1 \%$ \\
Fundament und Euter & $11,8 \%$ & $10,8 \%$ & $11,8 \%$ \\
\hline
\end{tabular}

In Anlehnung an diesen Zuchtwert führte der Schweizerische Braunviehverband im Jahr 2000 ein auf Schweizer Verhältnisse angepassten Ökologischen Gesamtzuchtwert ein (FiBL, 2001). Dieser Ökologische Gesamtzuchtwert wurde in Zusammenarbeit mit dem Forschungsinstitut für biologischen Landbau (FiBL) und BIO SUISSE weiterentwickelt und steht aktuell auch für die Rasse Holstein-Friesian zur Verfügung. Auch hier werden funktionale Merkmale stärker gewichtet als Produktionsmerkmale. Die männlichen Vererber der Rasse Braunvieh und Fleckvieh mit entsprechendem ÖZW, werden durch ein Kleeblatt gekennzeichnet und in etablierten Bullenkatalogen ausgewiesen. Die Einführung eines Ökologischen Gesamtzuchtwertes beim Schweizer Fleckviehzuchtverband, aber auch die Berechnung eines ÖZW für Kühe sowohl in Deutschland als auch in der Schweiz, ist noch in Bearbeitung. Postler (2002a) merkt dazu an, dass zukünftig mit Hilfe der Ökologischen Gesamtzuchtwerte eine länderübergreifende Zuchtarbeit ermöglicht werden kann. Ähnlich dem ÖZW in Deutschland wird in der Schweiz der Teilwert Leistung zu $40 \%$ und der Teilwert Konstitution zu 60 \% gewichtet. Eine vergleichbare Gewichtung ist nach Rozzi et al. (2007) im Ökologischen Gesamtzuchtwert für Kanada zu finden. Die Gewichtung für den Teilwert Leistung liegt hier sogar nur bei $28 \%$. Dementsprechend liegt das Gewicht für den Teilwert Konstitution bei $72 \%$. 


\subsection{Genotyp-Umwelt-Interaktionen zwischen konventionellen und ökolo- gisch wirtschaftenden Milchviehbetrieben}

Aus züchterischer Sicht stellt sich die grundlegende Frage, ob die Tiere, die unter den Bedingungen der konventionellen Landwirtschaft gezüchtet wurden, auch für den ökologischen Landbau geeignet sind. Bis zum jetzigen Zeitpunkt existieren vor allem Untersuchungen aus der konventionellen Landwirtschaft, die verschiedene Produktionsintensitäten (Pryce et al., 1999a) wie unterschiedliche Fütterungsstrategien bzw. die Nährstoffversorgung (Cromie et al., 1997; Pryce et al. , 1999b; Boelling et al., 2003), länderspezifische Umweltunterschiede (Chagunda et al., 1999; Costa et al., 2000; Zwald et al., 2003), aber auch Unterschiede zwischen Betriebstypen (Ron and Hillel, 1983; Boelling et al., 2003; König et al., 2005) betrachten. Untersuchungen bezüglich Genotyp-Umwelt-Interaktionen zwischen der konventionellen und ökologischen Milchviehzucht unterblieben aufgrund bislang nicht festgestellter direkter Unterschiede in der Genetik konventioneller und ökologischer Rinder (Boelling et al, 2003).

Als Genotyp-Umwelt-Interaktionen definiert Peters (1993) die (Un)Fähigkeit der Tiere, verschiedene genetische Potentiale eines Merkmals zu zeigen, wenn sie unterschiedlichen Umwelten ausgesetzt werden. Demnach können durch Veränderung des Umweltniveaus verschiedener Genotypen entsprechend Leistungsdifferenzen auftreten (Falconer, 1984). Aufgrund bestehender Haltungs- und Fütterungsunterschiede zwischen konventionellen und ökologischen Betrieben wird untersucht, inwieweit sich in konventionellen Betrieben geprüfte Zuchttiere, auch für Biobetriebe eignen. Werden dadurch Veränderungen in der Rangfolge bestimmter Genotypen hervorgerufen, besteht eine Genotyp-UmweltInteraktion (Schwarzenbacher, 2002; Fürst, 2006), welche bei der Zuchtplanung mitberücksichtigt werden sollte.

In der Studie von Kearney et al. (2004) wurden mögliche Genotyp-Umwelt-Interaktionen anhand der Leistungsmerkmale einer US-Holstein Population untersucht. Die Ergebnisse unter Weidebedingung wurden denen der Hochleistungsherden unter Stallbedingungen gegenübergestellt. Dabei fanden Kearney et al. (2004) heraus, dass die Bullen die in Hochleistungsherden getestet wurden, ihr genetisches Milchleistungspotential unter Weidebedingungen nicht entfalten konnten. Die verschiedenen Umweltbedingungen führten zu einer deutlich unterschiedlichen Rangierung einiger Bullen. Diese Differenzen und die genetischen Korrelationen von 0,89 für Milch, 0,88 für Fett und 0,91 für Eiweiß sind nach Meinung der Autoren nicht ausreichend, um für beide Systeme separate Zuchtwertschätzungen rechtfertigen zu können. Ihrer Meinung nach führt ein Produktionssystem mit nied- 
rigem Leistungsniveau aufgrund der Weidehaltung zu einer geringeren Sicherheit und zu Ungenauigkeiten bei der Selektion von Tieren. Auch bezweifeln Kearney et al. (2004), dass äußerst extensiv wirtschaftende Betriebe bereit sind, hohe Preise für Sperma von Bullen, die eine hohe Milchleistung vererben, zu zahlen. Ein gleiches Fazit zogen Weigel et al. (1999) aus ihrer Studie, in der sie Leistungsdaten amerikanischer Milchviehherden, die unter ähnlichen Fütterungsregimen wie bei Kearney et al. (2004) gehalten wurden, erhoben. Eine Herde wurde dabei unter intensiver Rotationsbeweidung und die Vergleichsherde unter konventionellen Bedingungen gehalten. Genetische Korrelationen zwischen den Merkmalen beider Betriebstypen von 0,92 für Milchmenge, 0,88 für Fettmenge und 0,99 für die Eiweißmenge weisen darauf hin, dass Genotyp-Umwelt-Interaktionen bei diesen Betriebsformen keine große Bedeutung haben. Außerdem sind die Zuchtwerte der unter konventionellen Bedingungen getesteten Bullen sicher genug, um diese auch für das Weidesystem nutzen zu können bzw. auch dort annähernd optimale Zuchtfortschritte zu erzielen. Die Analyse von Nauta et al. (2006b) zur Erhebung möglicher GenotypUmwelt-Interaktionen zwischen konventionellen und ökologischen Milchviehbetrieben in den Niederlanden basierte auf Erstlaktationsleistungen. Mit genetischen Korrelationen in den Bereichen von 0,79 bis 0,8 bei der Milchleistung, 0,86 bis 0,88 bei der Fettmenge und 0,71 bis 0,78 bei der Eiweißmenge, sind nur geringfügige Genotyp-Umwelt-Interaktionen zwischen den Mengenmerkmalen zu erkennen. Die Überlegung eines eigenen Zuchtprogramms für die ökologische Zucht auf Milchviehbetrieben würde nach Nauta et al. (2006b) erst dann interessanter werden, wenn die genetischen Korrelationen der Milchleistung deutlich unter 0,80 fallen würden.

Beim Vergleich zwischen konventionellen und ökologischen Milchviehbetrieben in Niederösterreich konnten nur sehr geringe Genotyp-Umwelt-Interaktionen festgestellt werden (Schwarzenbacher, 2002). Der Schwerpunkt dieser Studie lag in der Analyse der Milchleistung als Reaktion auf unterschiedliche Zusammensetzungen und Energiekonzentrationen der Futterrationen. Ergänzend zu dieser Studie untersuchte Fürst (2006) österreichische Fleckviehbetriebe, wobei die Väter der analysierten Kühe mindestens 30 Töchter in konventionellen und 10 Töchter in ökologischen Betrieben haben mussten. Auch hier wurden zur Schätzung der Genotyp-Umwelt-Interaktion die Milchleistung auf Bio- und konventionellen Betrieben 41.081 Laktationsleistungen der Jahre 2003 und 2005 untersucht. Mit einer genetischen Korrelation der Milchleistung von etwa 0,97 konnte jedoch auch keine Wechselwirkung zwischen den Betriebsarten nachgewiesen werden. Für die Merkmale Fett-\%, Eiweiß-\% und Zellzahl konnten aufgrund genetischer Korrelationen nahe 1 ebenfalls keine Genotyp-Umwelt-Interaktionen gefunden werden. 
Gerber et al. (2006) erhoben Genotyp-Umwelt-Interaktionen konventioneller und ökologisch wirtschaftender Fleckviehbetriebe in Bayern. Die konventionellen Betriebe wurden anhand des Haltungs- und Fütterungssystems in die Intensitätsstufen intensiv, sehr intensiv, extensiv und sehr extensiv unterteilt. Für die Datenanalyse standen Bullen der Geburtsjahrgänge 1993 bis 1994 mit jeweils mehr als 100 Töchtern zur Verfügung. Bullen die nicht in den Zweiteinsatz gekommen sind, wurden ausgeschlossen. Insgesamt setzte sich der Datenpool aus 278 Bullen mit 327.509 Töchtern zusammen. Zur Untersuchung der Genotyp-Umwelt-Interaktionen wurden Laktationsleistungen der ersten bis dritten Laktation herangezogen. Für die Milchleistung der ersten Laktation wurde eine genetische Korrelation von 0,94 festgestellt. Extremere Vergleiche der ökologischen Betriebe mit sehr intensiven und sehr extensiven Betrieben zeigten für die Milchmenge genetische Korrelationen von 0,99 bzw. 0,93. Die gleichen Betriebsvergleiche für das Merkmal Fett-kg zeigten genetische Korrelationen von 0,95, 0,98 und 0,92.

Der Vergleich ökologischer vs. konventioneller Betriebe zeigte für die Eiweißmenge eine genetische Korrelation von 0,88. Zwischen ökologisch und sehr intensiv lag die genetische Korrelation bei 0,98 und zwischen ökologisch und sehr extensiv lag diese bei 0,85. Die hier erbrachten Leistungen, zeigen aufgrund der hohen genetischen Korrelationen keine praxisrelevanten Genotyp-Umwelt-Interaktionen (Gerber et al., 2006). In Anlehnung an die Ergebnisse nach Weigel et al. (1999) und Kearney et al. (2004) zeigt auch diese Studie, dass sich die aktuellen Besamungsbullen, insbesondere die milchleistungsstarken Spitzenbullen, für alle Betriebsformen in gleicher Weise eignen. Alle in der Literatur beschriebenen genetischen Korrelationen für Leistungsmerkmale und funktionale Merkmale bei Schätzung der Genotyp-Umwelt-Interaktionen zwischen konventionellen und ökologischen Milchviehbetrieben, sind in Tabelle 2 und 3 zusammengefasst.

Tabelle 2: Genetische Korrelationen ( $\left.r_{g}\right)$ für die Milch-, Fett- und Eiweißmenge.

\begin{tabular}{lrrr}
\hline \multicolumn{1}{c}{ Quelle } & $r_{g}$ Milchmenge $[\mathrm{kg}]$ & $r_{g}$ Fettmenge $[\mathrm{kg}]$ & $r_{g}$ Eiweißmenge $[\mathrm{kg}]$ \\
\hline Weigel et al. (1999) & 0,92 & 0,88 & 0,99 \\
Kearney et al. (2004) & 0,89 & 0,88 & 0,91 \\
Nauta et al. (2006b) & $0,79-0,8$ & $0,86-0,88$ & $0,71-0,78$ \\
Fürst (2006) & 0,96 & & 0,88 \\
Gerber et al. (2006) & 0,94 & 0,95 & \\
\hline
\end{tabular}


Tabelle 3: Genetische Korrelationen $\left(r_{g}\right)$ für Fett-, Eiweißgehalt und Zellzahl.

\begin{tabular}{rrrr}
\hline Quelle & $r_{g}$ Fettgehalt [\%] & $r_{g}$ Eiweißgehalt [\%] & $r_{g}$ Zellzahl [Klasse] \\
\hline Fürst (2006) & 1,0 & 0,99 & 0,94 \\
\hline
\end{tabular}

Aktuelle Schätzungen der Genotyp-Umwelt-Interaktion für Leistungs- und funktionale Merkmale wurden im Rahmen des eigenen Projektes auch vom VIT anhand von Daten der Deutschen Holsteinzucht und vom FiBL und dem Applied Genetics Network (AGN) auf der Basis von Daten der Schweizer Braunvieh- und Fleckviehzucht durchgeführt. Die vorläufigen Ergebnisse sind in Tabelle 4 und Tabelle 5 dargestellt.

Tabelle 4: Vorläufige Ergebnisse der Genotyp-Umwelt-Interaktion zwischen konventionellen und ökologischen Betrieben in der Schweiz (Bapst und Stricker, 2006).

\begin{tabular}{lrr}
\hline & Braunvieh & Fleckvieh \\
\hline Anzahl Laktationen & 23.000 & 15.000 \\
$r_{g}$ Milchmenge & 0,85 & 0,96 \\
$r_{g}$ Fettmenge & 0,85 & 0,98 \\
$r_{g}$ Eiweißmenge & 0,90 & 0,97 \\
\hline
\end{tabular}

Anhand der Daten ist zu erkennen, dass sowohl in der Schweiz als auch in Deutschland für Milchleistungsmerkmale kaum Genotyp-Umwelt-Interaktionen bestehen. Vom VIT wurden bereits Untersuchungen zu funktionalen Merkmalen wie Zellzahl und Nutzungsdauer durchgeführt. Die für die Zellzahl ermittelten niedrigen genetischen Korrelationen im Bereich von 0,57 und 0,27 und die noch geringeren Korrelationen bei der Nutzungsdauer, deuten darauf hin, dass Genotyp-Umwelt-Interaktionen bei den funktionalen Merkmalen existieren. 
Tabelle 5: Vorläufige Ergebnisse der Genotyp-Umwelt-Interaktion zwischen konventionellen und ökologischen Betrieben in Deutschland (Reinhardt, 2006).

\begin{tabular}{lrr}
\hline & ökologisch & konventionell \\
\hline Anzahl Töchter & 36.147 & 4,2 Mio. \\
Anzahl Väter (> 5 Töchter) & 1334 & 1334 \\
Anzahl Betriebe & 179 & 37.661 \\
\hline
\end{tabular}

\begin{tabular}{lccr}
\hline & 1. Lakt. & 2. Lakt. & 3. Lakt. \\
\hline$r_{g}$ Milchmenge & 0,91 & 0,91 & 0,87 \\
$r_{g}$ Fettmenge & 0,84 & 0,83 & 0,72 \\
$r_{g}$ Eiweißmenge & 0,90 & 0,89 & 0,83 \\
$r_{g}$ Zellzahl & 0,57 & 0,55 & 0,27 \\
\hline
\end{tabular}

Experten der ökologischen Tierzucht fordern aufgrund der zukünftigen Zuchtziele mit Schwerpunkt auf den funktionalen Merkmalen weitere umfassende Untersuchungen zu Genotyp-Umwelt-Interaktionen (Nauta et al., 2001, Bijma et al., 2002). Auch Fürst (2006) erwähnt die Notwendigkeit, weitere Analysen zu Genotyp-Umwelt-Interaktionen im Bereich der Fitnessmerkmale, u. a. der Nutzungsdauer, durchzuführen.

\subsection{Zuchtplanung und Optimierung von Zuchtprogrammen}

Zuchtplanungsrechnungen werden durchgeführt, um den Effekt von Zuchtmaßnahmen zu quantifizieren und den Erfolg von Zuchtprogrammen zu optimieren. Nach Gierzinger (2002) können Simulationsmodelle zur Zuchtplanung nicht nur der Alternativensuche dienen, sondern auch als Prognosehilfsmittel. Diese sind ebenso in der Lage, die Wirkung eines Zuchtprogramms wie den zu erwartenden züchterischen Erfolg, Ertrag und Aufwand eines alternativen Zuchtprogramms abzuschätzen und Trends aufzuzeigen. Das Computerprogramm ZPLAN von Nitter et al. (2000) ermöglicht die Erstellung und Bewertung verschiedener züchterischer Strategien. Neben der Optimierung von Zuchtprogrammen gibt dieses Programm Entscheidungshilfen für viele Zuchtplanungsfragen und beruht auf einem rein deterministischen Ansatz. Zentraler Bestandteil von ZPLAN ist die Genflussmethode. 
Mit Hilfe der Genflussmethode können viele Fragestellungen der Tierzucht und Zuchtplanung beantwortet werden, wie z. B. die Berechnung des Züchtungsertrages. Die klassische Formel des Zuchtfortschrittes nach Rendel und Robertson (1950) ist die Grundlage der Entstehung der Genflussmethode. Da die Überlappung der Generationen nicht berücksichtigt und der Zeitpunkt und die Häufigkeit der Realisierung des Zuchtfortschrittes in der klassischen Berechnung nicht beachtet wird, wurde die von McClintock und Cunningham (1974) entwickelte und durch Hill (1974) in eine vereinfachte Matrixschreibweise gebrachte Genflussmethode entwickelt, die den Fluss der Gene relativ einfach und übersichtlich beschreibt und sowohl die Berechnung des Zuchtfortschrittes als auch die des diskontierten Züchtungsertrages bei überlappenden Generationen erlaubt. Der Züchtungsertrag dient der Evaluierung von Zuchtprogrammen und ist für die Beurteilung der Ergebnisse einzelner Selektionsmaßnahmen von großer Bedeutung.

Untersuchungen zu Zuchtplanungsrechnungen für die Rinderzucht im ökologischen Landbau, wurden im deutschsprachigen Raum bislang nur von Kalm und Harder (2003) durchgeführt. Auf Basis einer Befragung von ökologischen Verbänden, Rinderzuchtorganisationen sowie Vermarktungs- und Verarbeitungsbetrieben wurden verschiedene Zuchtprogrammvarianten unter Anwendung des Computerprogramms ZPLAN durchgeführt. Das Ziel der Studie von Kalm und Harder (2003) lag, verglichen zur vorliegenden Studie, ebenfalls darin, anhand einer Reihe alternativer Zuchtprogramme die Notwendigkeit eines eigenen ökologischen Zuchtprogramms zu untersuchen. Weiterhin sollte überprüft werden, ob bereits bestehende Konzepte der konventionellen Rinderzucht durch entsprechende Anpassungen optimiert werden können, um den ökologischen Anforderungen zu genügen (Kalm und Harder, 2003). Aus den Ergebnissen der in ZPLAN untersuchten Parameter, dem fehlenden Interesse seitens der Zuchtorganisationen und den hohen Kosten die bei der Entwicklung eines solchen Zuchtprogramms entstehen würden, kommen Kalm und Harder (2003) zu dem Schluss, dass kein Bedarf für ein eigenes ökologisches Zuchtprogramm besteht. 


\section{Datenmaterial und Auswertungsmethodik}

\subsection{Datenmaterial ökologischer Milchviehbetriebe in Deutschland}

Für die Analysen der genetischen und phänotypischen Trends bei Bullen und Kühen auf deutschen ökologischen Milchviehbetrieben, des ET-Status der Bullen und deren genetischen Niveaus in den Relativzuchtwerten standen Daten von 442 ökologisch wirtschaftenden Betrieben zur Verfügung. Diese wurden zuvor mit Hilfe des Thüringer Ökoherz und dem VIT identifiziert und vom VIT im Januar 2006 bereitgestellt (Tabelle 6).

Tabelle 6: Darstellung des gesamten Datenmaterials und die Auswahl der zur Berechnung geeigneten Daten.

\begin{tabular}{|c|c|}
\hline Datei & Datensatz \\
\hline \multirow[t]{10}{*}{ Leistungsdaten } & 44.234 Leistungsrecords gesamt, davon \\
\hline & 37.878 Records von Holsteinkühen; \\
\hline & nach entsprechenden Plausibilitätsprüfungen: \\
\hline & 24.135 Kühe aller Rassen, davon \\
\hline & 20.739 Holsteinkühe \\
\hline & Phänotypischer Trend: \\
\hline & 44.234 Leistungsrecords gesamt, davon \\
\hline & $\begin{array}{l}42.469 \text { Records nach Ausschluss von Tieren bei denen einzelne Leis- } \\
\text { tungen nicht vorhanden waren, davon }\end{array}$ \\
\hline & 36.313 Records von Holsteinkühen, davon \\
\hline & $\begin{array}{l}36.015 \text { Records, bei denen die definierten Laktationsklassen ausrei- } \\
\text { chend besetzt sind }\end{array}$ \\
\hline \multirow[t]{5}{*}{ Abstammungsdaten } & 24.257 Kühe mit Leistung, davon \\
\hline & 8.401 Kühe mit Leistung und als Mutter \\
\hline & 84.552 Bullen mit Record im Pedigree File \\
\hline & 78.390 Ahnen \\
\hline & 102.647 Tiere insgesamt \\
\hline Exterieurdaten & 1.890 Exterieurdaten \\
\hline Besamungsdaten & 57.527 Besamungsdatensätze \\
\hline Zuchtwerte Kühe & 24.257 Records \\
\hline
\end{tabular}


Die Betriebsdaten sind in fünf Dateien, den Leistungs-, Abstammungs-, Exterieur-, Besamungs- und Zuchtwertdaten, eingeteilt. Der Rohdatenumfang und der Datenumfang nach den für die folgenden Untersuchungen nötigen Plausibilitätsprüfungen sind in Tabelle 6 dargestellt. Die Statistische Auswertung der folgenden Untersuchungen wurde mit dem Programm SAS 9.1 durchgeführt.

\subsubsection{Genetische und phänotypische Trends der Leistungs- und funktionalen Merkmale bei Bullen und Kühen ökologischer Betriebe}

Im Rahmen der Bachelorarbeit von Stratmann (2006) wurden die vom VIT bereitgestellten Daten auf genetische und phänotypische Trends für Leistungs- und funktionalen Merkmale in ökologisch wirtschaftenden Milchviehbetrieben untersucht. Dazu wurden überwiegend die Informationen aus der Zuchtwertdatei der Kühe der Ökobetriebe, deren Leistungsdatei und Abstammungsdatei benötigt. Weiterhin wurden die Zuchtwerte Schwarzbunter Bullen aus der Zuchtwertschätzung August 2005 verwendet, um die genetischen Trends der Bullen berechnen zu können. Auch bei den Kühen werden in dieser Untersuchung ausschließlich Daten Schwarzbunter Kühe berücksichtigt.

Für den Vergleich von konventionellen zu ökologischen Bullen wurden vorab die Ohrmarkennummern der Väter der Kühe aus der Abstammungsdatei ökologischer Betriebe mit den Ohrmarkennummern der Bullen aus der Zuchtwertdatei August 2005 verglichen. Die Bullen, die sowohl in der Abstammungsdatei der „Ökokühe“, Kühe auf ökologischen Betrieben, als auch in der Zuchtwertdatei der konventionellen Bullen zu finden waren, werden in dieser Arbeit als „Ökobullen“ bezeichnet. Die Vergleichsgruppe der konventionellen Bullen bilden alle der in der Zuchtwertdatei enthaltenen Bullen, einschließlich der auch als Ökobullen ausgewiesenen Tiere. Die genetischen Trends werden bei dieser Untersuchung ausschließlich auf der Basis von geschätzten Zuchtwerten errechnet. Im Rahmen der Analyse der Leistungs- und funktionalen Merkmale bei den Kuhvätern werden nach entsprechenden Plausibilitätsprüfungen Zuchtwerte von insgesamt 8.676 konventionellen Bullen mit Zuchtwerten von 1.645 Ökobullen innerhalb eines Betrachtungszeitraums von 1993 bis 2000 verglichen. Der genetische Trend bei den Ökobullen wurde in dem Programm SAS 9.1 durch Zusammenspielen der ökologischen Zuchtwert-, Leistungs- und Abstammungsdatei mit der Zuchtwertdatei für Bullen ausgegeben. Die entsprechenden Informationen zur Ermittlung der genetischen Trends bei den konventionellen Bullen konnten der Zuchtwertdatei August 2005 entnommen werden. Der genetische Fortschritt wird als durchschnittlicher Wert eines betrachteten Geburtsjahres der Bullen dargestellt. 
Für die genetischen Trends bei den Ökokühen werden die Jahrgänge 1994 bis 2003 betrachtet. Auch bei dieser Untersuchung dienten die Zuchtwertdatei, Leistungs- und Abstammungsdatei der ökologischen Betriebe als Berechnungsgrundlage, um die genetischen Trends zu bestimmen. Dabei standen für den Betrachtungszeitraum 1993 bis 2003 Zuchtwertdaten von 24.135 Ökokühen aller Rassen zur Verfügung. Durch eine weitere Einschränkung auf ausschließlich Schwarzbunte Kühe wurde die Anzahl Kühe mit Zuchtwertinformationen auf 20.739 Tiere reduziert. Auch hier wurde eine Übersicht über den durchschnittlichen genetischen Zuchtfortschritt der Merkmale und Relativzuchtwerte pro untersuchtes Geburtsjahr der Kühe erstellt. Die phänotypischen Trends, welche sich aus erbrachten Ergebnissen wie der Laktationsleistung der Kuh ergeben, werden, anders als bei den genetische Trends, erst ab dem Jahrgang 1995 bis zum Jahr 2003 betrachtet.

Der phänotypische Trend der Ökokühe für die Einsatzleistung in verschiedenen Laktationsklassen wurde nur von der ersten bis zur dritten Laktation ausgewertet. Unter diesen Bedingungen und nach einer Sortierung der Daten nach den Geburtsjahrgängen 1995 bis 2003 standen 36.015 Datensätze für die phänotypische Leistung von Ökokühen der Rasse Schwarzbunt zur Verfügung. Für alle Rassen sind 42.469 Datensätze mit phänotypischen Leistungen vorhanden. Schwarzbunte Kühe nehmen infolgedessen einen Anteil von $85 \%$ am Gesamtdatensatz der phänotypischen Leistungen ein. Der phänotypische Trend wurde als Regression des Geburtsjahres auf die durchschnittliche Milchleistung innerhalb einer Laktation berechnet.

\subsubsection{Untersuchung zum ET-Status und Erhebung genetischer Trends ökologi- scher Bullen im Vergleich zu konventionellen Bullen}

Für diese Untersuchungen wurden die Bullen- und Kuhinformationen aus vier Dateien benötigt und in SAS 9.1 zusammengespielt. Der Rechenlauf enthält neben den Zuchtwertdaten, den Abstammungsdaten und den Besamungsdaten der ökologischen Betriebe auch konventionelle Zuchtwertdaten der Zuchtwertschätzung August 2005, um den gewünschten Vergleich herzustellen. Informationen über die ET-Kennzeichnung sind in der Besamungsdatei enthalten. Ebenso enthält diese Datei Informationen darüber, ob es sich bei den Kuhvätern bzw. Anpaarungsbullen um Wiedereinsatzbullen, Test- oder Deckbullen handelt. Für die Untersuchungen wurde der Datensatz so editiert, dass jede Kuh und jeder Bulle nur einmal betrachtet wurden. Der gesamte ausgewertete Datenpool enthält neben Schwarz- und Rotbunten Holsteins auch Rassen wie Jersey, Angler, Rotvieh, Angler alte Zuchtrichtung, Rotbunt Doppelnutzung, Schwarzbunt Genreserve mit niedrigem Holstein-Friesian Anteil, Angler mit der Zuchtrichtung Höhenvieh und fünf diverse andere 
Kreuzungen. Diese Rassen werden in der Auswertung als „sonstige bzw. alle“ Rassen gekennzeichnet und den Ergebnissen der Auswertungen speziell für die Rasse Schwarzund Rotbunte Holsteins gegenübergestellt. Ergänzend dazu muss jedoch erwähnt werden, dass der Anteil HF unter allen in der Berechnung betrachteten Rassen $96 \%$ ausmacht (s. Abbildung 5).

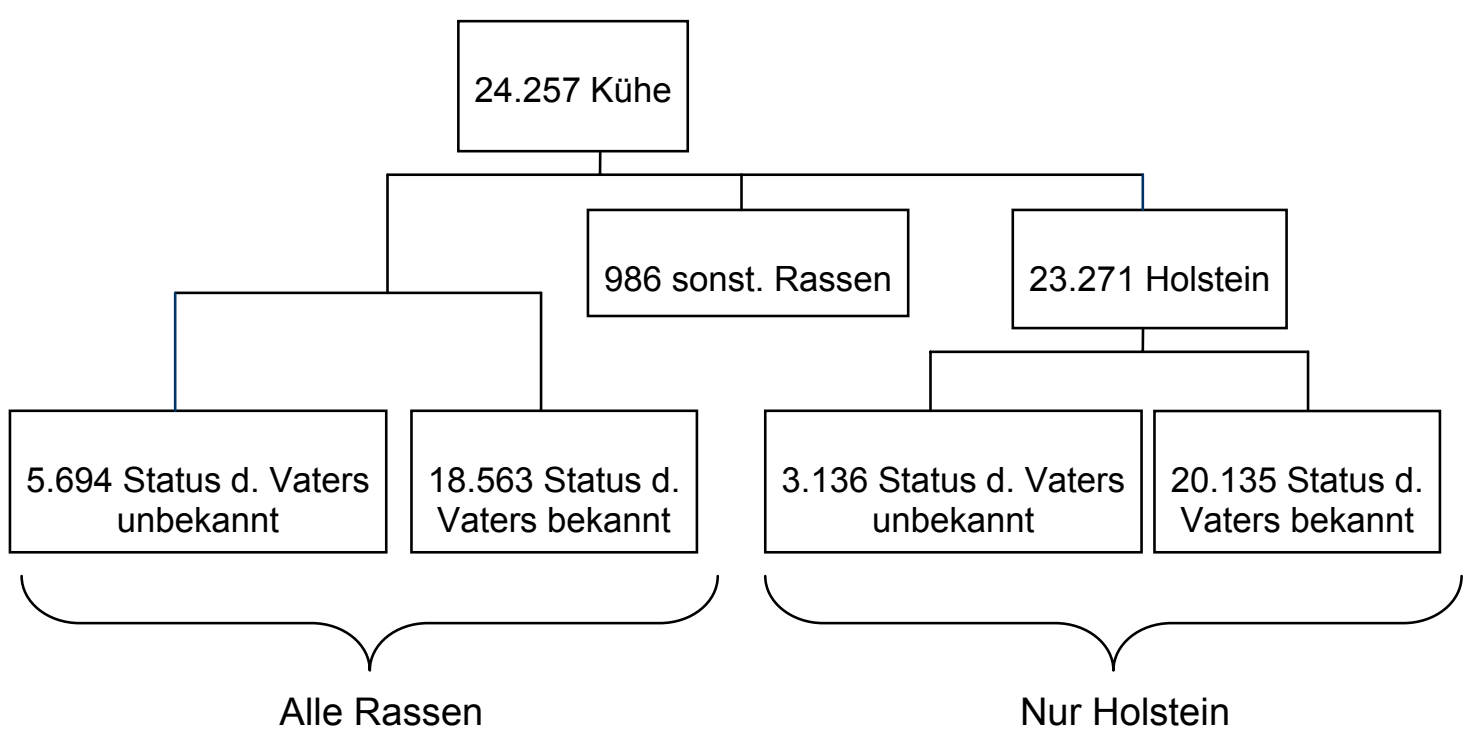

Abbildung 5: Datenmaterial zur Analyse des Status der auf ökologischen Betrieben eingesetzten Kuhväter.

Abbildung 5 zeigt den Aufbau und die untersuchungsspezifische Aufteilung des KuhDatenpools. Die Gesamtzahl Kühe setzt sich aus 23.271 Tieren der Rasse Schwarz- und Rotbunte Holsteins und aus 986 Kühen der oben beschriebenen übrigen Rassen zusammen. Diese Aufteilung ist in der mittleren Ebene des Diagramms abgebildet. Sowohl der gesamte Datenpool als auch die herausgefilterten Holstein-Kühe werden auf den ETStatus ihrer Väter untersucht und miteinander verglichen. In der nächsten Diagrammebene werden wiederum der gesamte Kuh-Datenpool und die Daten der Holstein-Kühe in die Bullenkategorien Test-, Deck oder Wiedereinsatzbulle differenziert. Bei 18.563 von insgesamt 24.257 Kühen ist der Status des Vaters bekannt und bei 5.694 Kühen unbekannt. Der Pfad der Rasse Holstein-Friesian zeigt 20.135 Kühe mit Angaben zum Status des Vaters und 3.136 Kühe ohne Angaben. 
In der Analyse wird der ET-Status der Kuhväter nur für die Ökokühe der Geburtsjahrgänge 1994 bis 2003 betrachtet. Für den ET-Status der Anpaarungsbullen der Ökokühe werden die Belegungsjahre von 1996 bis 2005 untersucht. Bei beiden Untersuchungen wird die Anzahl Kühe pro Geburts- bzw. Belegungsjahr ausgewiesen. Die Kategorisierung der Kuhväter nach Wiedereinsatz-, Test- oder Deckbullen wird für die Jahrgänge 1994 bis 2003 dargestellt und bei den Anpaarungsbullen für die Belegungsjahre 1996 bis 2005

Ferner wurde die Entwicklung des genetischen Niveaus der zuvor identifizierten ökologischen ET- und Nicht-ET-Kuhväter ausgewertet. Diese Untersuchung wurde jedoch nur für die Bullen der Rasse Holstein-Friesian durchgeführt. Pro betrachtetem Geburtsjahr der Väter erfolgte eine Unterteilung in ET- und Nicht-ET-Kuhvätern. Innerhalb dieser Unterteilung wurden Zuchtwerte für die gewählten Merkmale aufgelistet. Im Ergebnisteil sind demzufolge die Trends der Relativzuchtwerte Milchleistung (RZM), Zellzahl (RZS), Exterieur (RZE), Zuchtleistung (RZZ), Nutzungsdauer (RZN) und des Gesamtzuchtwertes (RZG) dargestellt. Die Informationen zu den Zuchtwerten stammen aus den Geburtsjahrgängen 1993 bis 2000 .

\subsection{ZPLAN - Modellkalkulationen zum Zuchtfortschritt für ein ökologisches Zuchtprogramm}

Um für die nachhaltige Milchviehzucht im ökologischen Landbau verschiedene züchterische Strategien aufstellen und bewerten zu können, werden alle Modellrechnungen in dieser Arbeit mit dem Computerprogramm ZPLAN von Nitter et al. (2000) durchgeführt. Um mit dem Computerprogramm ZPLAN verschiedene Zuchtplanungsrechnungen durchführen zu können, müssen vorab im Input eine Reihe von Parametern für die zu untersuchende Population definiert werden. Diese Parameter werden vom Benutzer so gewählt, dass das Zuchtprogramm möglichst realistisch abgebildet wird. Dies betrifft Parameter der Populationsstruktur, biologisch-technische Parameter und Kostenparameter. Nach Berechnung einzelner Zuchtprogrammvarianten, kann ein für das jeweilige Vorhaben optimale Zuchtprogramm bestimmt werden. Jedoch muss bei der Wahl der Parametergrößen mit berücksichtigt werden, dass das Programm ZPLAN nicht für Extremwerte geeignet ist (Willam, 2007). Bei der Untersuchung sollte darauf geachtet werden, dass nur Variablen in einem realistischen, umsetzbaren Bereich variiert werden (Willam, 2007). 


\subsubsection{Inputparameter zur Beschreibung der Populationsstruktur des gewählten Zuchtprogramms}

Die wichtigsten Input-Parameter, die den Aufbau des ökologischen Zuchtprogramms beschreiben, werden in den folgenden Kapiteln beschrieben und in den Tabelle 7 bis Tabelle 10 dargestellt.

\subsubsection{Parameter zur Beschreibung der Ökopopulation}

Um die Ausgangspopulation des Zuchtprogramms möglichst realistisch abzubilden, wurde auf Erhebungen des Statistischen Bundesamtes Deutschland zurückgegriffen. Demzufolge besteht die vorliegende Ausgangspopulation aus 109.600 Kühen (DESTATIS, 2005). Alle anderen Werte wurden für das ökologische Zuchtprogramm angenommen oder Literaturstudien entnommen und sind in Tabelle 7 aufgeführt.

Tabelle 7: Die wichtigsten Parameter zur Beschreibung der Populationsstruktur im ökologischen Basiszuchtprogramm.

\begin{tabular}{lcr}
\hline Populationsparameter & Einheit & Wert \\
\hline Populationsgröße Kühe & Anzahl Tiere & 109.600 \\
Kuhanteil in der Zuchtstufe & $\%$ & 80 \\
Kuhanteil in der Produktionsstufe & $\%$ & 20 \\
KB-Anteil in der Zuchtstufe & $\%$ & 88 \\
KB-Anteil in der Produktionsstufe & $\%$ & 80 \\
Testanteil & $\%$ & 25 \\
Remontierung der Testbullen & Anzahl Tiere & 20 \\
Testbullen & Anzahl Tiere & 20 \\
Altbullen & Anzahl Tiere pro Jahr & 20 \\
ökologische Testbullen-Väter & Anzahl Tiere pro Jahr & 2 \\
konventionelle Testbullen-Väter & \% & 2 \\
Anteil Besamungen mit konventionellen Altbullen & Anzahl Besamungen & 12,5 \\
Besamungen je Tochterleistung & & 10 \\
\hline
\end{tabular}




\subsubsection{Biologisch-technische Parameter}

Die Parameter, um die Nutzungsdauer und das Alter der Tiere, Abkalbe- und Aufzuchtverluste, das durchschnittliche Generationsintervall, die Zwischenkalbezeit und den Besamungsindex zu beschreiben, wurden in Anlehnung an die Populationsparameter angenommen oder z. T. Literaturstudien entnommen. Tabelle 8 zeigt eine Übersicht über die gewählten biologisch-technischen Parameter, die für das vorliegende Zuchtprogramm angenommen wurden.

Tabelle 8: Die wichtigsten biologisch-technischen Parameter für den Basislauf des ökologischen Zuchtprogramms.

\begin{tabular}{lcr}
\hline Biologisch-technische Parameter & Einheit & Wert \\
\hline Zwischenkalbezeit & Jahre & 1,07 \\
Besamungsindex & Besamungen pro Trächtigkeit & 1,7 \\
Abkalbeverluste & $\%$ & 4 \\
Aufzuchtverluste weiblich & $\%$ & 15 \\
Aufzuchtverluste männlich & $\%$ & 25 \\
Nutzungsdauer Testbulle & Jahre \\
Nutzungsdauer Altbullen & Jahre & 0,25 \\
Nutzungsdauer Testbullen-Väter & Jahre \\
Nutzungsdauer Testbullen-Mütter & Jahre & 2,0 \\
Nutzungsdauer Kühe Zuchtstufe & Jahre & 1,0 \\
Nutzungsdauer Kühe Produktionsstufe & Jahre & 3,0 \\
Alter der Testbullen-Väter & Jahre & 3,8 \\
Alter der Altbullen & Jahre & 4,0 \\
Alter der Kühe in der Zuchtstufe & Jahre & 6,5 \\
Alter der Testbullen-Mütter & Jahre & 7,0 \\
Durchschnittliches Generationsintervall & Jahre & 2,6 \\
\hline
\end{tabular}

\subsubsection{Parameter zur Beschreibung der Kosten}

Eine Übersicht über die anfallenden Kosten für das modellierte Zuchtprogramm ist in Tabelle 9 dargestellt. Um die degressive Kostenstruktur mit steigender Populationsgröße zu berücksichtigen, wurden die variablen und fixen Zuchtprogrammkosten von zwei realen 
Zuchtverbänden übernommen. Durch den starken Unterschied in der Anzahl an Herdbuchkühen ergeben sich für den Zuchtverband A mit 253.416 Herdbuchkühen und $3.156 €$ pro Kuh und Jahr insgesamt Fixkosten in Höhe von etwa $800.000 €$ pro Jahr. Für den zweiten Zuchtverband B mit nur 91.137 Herdbuchkühen in der Zuchtstufe fallen ca. $330.372 €$ pro Jahr an. Diese Summe ergibt sich aus der Annahme, dass pro Kuh und Jahr etwa $3.625 €$ benötigt werden. Der Unterschied in den Beträgen der Jahreskosten pro Kuh ist darauf zurückzuführen, dass mit steigender Anzahl an Kühen in der Zuchtstufe eine Kostendegression eintritt. Für die restlichen Werte wurden wiederum möglichst realistische Annahmen getroffen bzw. auf Literaturstudien zurückgegriffen.

Tabelle 9: Die wichtigsten Kostenparameter für den Basislauf des Zuchtprogramms für die ökologische Milchviehhaltung.

\begin{tabular}{lcr}
\hline Kostenparameter & Einheit & Wert \\
\hline Milchleistungsprüfung pro Kuh und Jahr & $€$ & 31,00 \\
Selektion Testbullen-Mütter & $€$ & 66,00 \\
Selektion Testbullen-Väter & $€$ & 648,00 \\
Gezielte Paarung & $€$ & 20,00 \\
Selektion Kälber & $€$ & 12,50 \\
Eigenleistungsprüfung Bulle auf Station & $€$ & 1286,00 \\
Führung Zuchtbuch pro Zuchttier & $€$ & 0,96 \\
Bewertung Erstlingskuh für Zuchtbuch & $€$ & 11,00 \\
Produktion Spermaportion & $€$ & 0,80 \\
Lagerung Spermaportion & $€$ & 0,03 \\
Wartebullenhaltung & $€$ & 2647,00 \\
Mehrkosten konventioneller Spermaportionen & $€$ & 5,00 \\
Variable Kosten pro Testbulle & $€$ & 3044,00 \\
\hline
\end{tabular}

\subsubsection{Investitionsparameter}

Das erstellte ökologische Zuchtprogramm wird auf eine Gesamtlaufzeit von 20 Jahren berechnet. Dadurch ergibt sich für jeden einzelnen Kostenparameter eine Diskontierung innerhalb der angesetzten Investitionsperiode. Für die während dieser Laufzeit aufkommenden Kosten wird ein Zinssatz von 4 \% gewählt. Der Zinssatz für den Gewinn bzw. Ertrag beträgt $6 \%$ (vgl. Tabelle 10). 
Tabelle 10: Investitionsparameter des Basiszuchtprogramms.

\begin{tabular}{lcr}
\hline Investitionsparameter & Einheit & Wert \\
\hline Zinssatz für den Ertrag & $\%$ & 6 \\
Zinssatz für die Kosten bzw. Aufwand & $\%$ & 4 \\
Investitionsperiode & Jahre & 20 \\
\hline
\end{tabular}

\subsubsection{Die Genflussmatrix für das ökologische Zuchtprogramm}

In diesem Bereich wird festgelegt wie die Gene von Tieren unterschiedlicher Alters- und Geschlechtsgruppen pro Selektionsrunde durch Alterung und Reproduktion auf die direkten Nachkommen übertragen werden. Die Selektionsrunde beschreibt alle Selektionsmaßnahmen, die sich auf die Tiere eines Jahrgangs von der Geburt bis zur letzten Zuchtverwendung beziehen. Durch die modellhafte Aufteilung der Population in eine Zucht- und Produktionsstufe, ergeben sich so genannte Selektionsgruppen. Tiere, die den gleichen Selektionsmaßnahmen unterliegen, gehören einer Gruppe an. Durch diese Stufeneinteilung kann die Ausbreitung der Gene auf die gesamte Population untersucht werden. Für die eigene Untersuchung wurde die vorliegende Population in 19 verschiedene Selektionsgruppen aufgeteilt.

Der Genfluss für ein ökologisches Zuchtprogramm ist in Tabelle 11 dargestellt. Da neben dem Genfluss der Bullen aus ökologischer Abstammung auch der Genfluss von konventionellen Besamungsbullen für dieses Zuchtprogramm berücksichtigt wird, handelt es sich im Gegensatz zu Kalm und Harder (2003) nicht um ein geschlossenes ökologisches Zuchtprogramm. In der Basisvariante wurde der Genanteil der konventionellen Altbullen auf einen Wert von $16 \%$ fixiert. 
Tabelle 11: Darstellung der 19 verschiedenen Selektionsgruppen und Genflüsse zur Übertragung des genetischen Fortschritts.

\begin{tabular}{|c|c|c|c|c|c|c|}
\hline & HK-Ö & KB-ö & NS-Ö & KB-k & HK-k & NHK-ö \\
\hline HK-ö & 1.HK-ö>HK-ö & $\begin{array}{l}\text { 2. TB-ö }>\text { HK-ö } \\
\text { 3.AB-ö* }>\text { HK-ö }\end{array}$ & 4.NS-ö>HK-ö & $5 . A B-k^{* *}>\mathrm{HK}-0 ̈$ & & \\
\hline KB-ö & 6.HK-ö>AB-ö* & 7.AB-ö* $>$ AB-ö $*$ & & 8.AB-k ${ }^{\star \star}>A B-0 ̈$ & & \\
\hline NS-ö & 9.HK-ö>NS-ö & $\begin{array}{l}\text { 10.TB-ö* }>\text { NS-ö } \\
\text { 11.AB-ö̈* }>\text { NS-ö }\end{array}$ & & 12.AB- $k^{* *}>\mathrm{NS}-\mathrm{O}$ & & \\
\hline KB-k & & & & 13.AB- $-k^{* *}>A B-k^{* *}$ & 14. HK-k>AB-k & \\
\hline HK-k & & & & 15.AB-k**HK-k & 16. HK-k>HK-k & \\
\hline NHK-ö & & 17.AB-ö ${ }^{*}>$ NHK-ö & 18.NS-ö>NHK-ö & & & 19.NHK-ö>NHK-ö \\
\hline
\end{tabular}

HK-ö = Herdbuchkühe ökologisch - Kühe der Zuchtstufe

KB-ö = Künstliche Besamung - Bullen der ökologischen Zuchtstufe

* $($ TB-ö $=$ Testbullen ökologisch, AB-ö = Altbullen ökologisch $)$

NS-ö = Natursprung ökologisch - Bullen der ökologischen Zuchtstufe

$\mathrm{KB}-\mathrm{k}=$ Künstliche Besamung - konventionelle Bullen

${ }^{* *}(\mathrm{AB}-\mathrm{k}=$ Altbullen konventionell)

$\mathrm{HK}-\mathrm{k}=\mathrm{Kühe}$ der Zuchtstufe konventionell

NHK-ö = Nicht-Herdbuchkühe ökologisch - Kühe der Produktionsstufe

Die Selektionsgruppen 1 bis 16 zeigen den Ablauf der Genflüsse in der Zuchtstufe bzw. im Zuchtbereich. Die Gruppen 17 bis 19 beschreiben die Genübertragung in der Produktionsstufe. Gemäß der vorliegenden Genübertragungsmatrix gibt es 19 verschiedene Genflüsse zur Übertragung des genetischen Fortschritts. Diese werden in Tabelle 12 auf der folgenden Seite erläutert. Die einzelnen 19 Selektionsgruppen tragen zum Zuchtfortschritt und Züchtungsgewinn in der Gesamtpopulation bei. Mittels Selektionsindexkalkulation gilt es, den Zuchtfortschritt für den Gesamtzuchtwert als auch für die Einzelzuchtwerte, die das Zuchtziel beschreiben, zu quantifizieren. Für jede der 19 Selektionsgruppen muss somit im Detail festgelegt werden, welche Informationsquellen (Information im Index) zum Zuchtwert beitragen. 
Tabelle 12: Darstellung der Genflüsse in den 19 Selektionsgruppen.

\begin{tabular}{clll}
\hline Nr. & Gruppe & Übertragung der Gene von... & auf... \\
\hline 1. & HK-ö $>$ HK-ö & Kühen der ökolog. Zuchtstufe & Kühe der ökolog. Zuchtstufe \\
2. & TB-ö $>$ HK-ö & ökologischen Testbullen & Kühe der ökolog. Zuchtstufe \\
3. & AB-ö $>$ HK-ö & ökologischen Altbullen & Kühe der ökolog. Zuchtstufe \\
4. & NS-ö $>$ HK-ö & ökologischen Natursprungbullen & Kühe der ökolog. Zuchtstufe \\
5. & AB-k $>$ HK-ö & konventionellen Altbullen & Kühe der ökolog. Zuchtstufe \\
6. & HK-ö $>$ AB-ö & Kühen der ökolog. Zuchtstufe & ökologische Altbullen \\
7. & AB-ö $>$ AB-ö & ökologischen Altbullen & ökologische Altbullen \\
8. & AB-k $>$ AB-ö & konventionellen Altbullen & ökologische Altbullen \\
9. & HK-ö $>$ NS-ö & Kühen der ökolog. Zuchtstufe & ökologische Natursprungbullen \\
10. & TB-ö $>$ NS-ö & ökologischen Testbullen & ökologische Natursprungbullen \\
11. & AB-ö $>$ NS-ö & ökologischen Altbullen & ökologische Natursprungbullen \\
12. & AB-k $>$ NS-ö & konventionellen Altbullen & ökologische Natursprungbullen \\
13. & AB-k $>$ AB-k & konventionellen Altbullen & konventionelle Altbullen \\
14. & HK-k $>$ AB-k & Kühen der konven. Zuchtstufe & konventionelle Altbullen \\
15. & AB-k $>$ HK-k & konventionellen Altbullen & Kühe der konven. Zuchtstufe \\
16. & HK-k $>$ HK-k & konven. Kühen der Zuchtstufe & Kühe der konven. Zuchtstufe \\
17. & AB-ö $>$ NHK-ö & ökologischen Altbullen & Kühe der ökolog. Prod.stufe \\
18. & NS-ö $>$ NHK-ö & ökologischen Natursprungbullen & Kühe der ökolog. Prod.stufe \\
19. & NHK-ö $>$ NHK-ö & Kühen der ökolog. Prod.stufe & Kühe der ökolog. Prod.stufe \\
\hline & & & \\
\hline
\end{tabular}

\subsubsection{Zuchtwertschätzung mittels Selektionsindex}

Für eine Zuchtwertschätzung mittels Selektionsindex gilt es, die genetische Kovarianzmatrix zwischen Merkmalen im Zuchtwert und Merkmalen im Index sowie die phänotypische Varianz- Kovarianzmatrix der Indexmerkmale zu erstellen. Die für das ökologische Zuchtprogramm gewählten Heritabilitäten und genetische sowie phänotypische Korrelationen der Merkmale wurden den Studien von Gierzinger (2002) und Gredler (2004) entnommen und sind in Tabelle 13 zusammengestellt. 
Tabelle 13: Phänotypische (oberhalb der Diagonalen) und genetische Korrelationen (unterhalb der Diagonalen) und Heritabilitäten (Diagonale) der Merkmale im Index.

\begin{tabular}{lllllllllllll}
\hline Merkmal & Fkg & Ekg & ND & Pers & FRUp & FRUm & KVLp & KVLm & TOTp & TOTm & ZZ & DMG \\
\hline Fkg & $\mathbf{0 . 3 8}$ & 0.8 & 0.0 & 0.0 & 0.0 & 0.0 & 0.0 & 0.0 & 0.0 & 0.0 & 0.0 & 0.2 \\
Ekg & 0.85 & $\mathbf{0 . 3 4}$ & 0.0 & 0.0 & 0.0 & 0.0 & 0.0 & 0.0 & 0.0 & 0.0 & 0.0 & 0.2 \\
ND & -0.1 & -0.1 & $\mathbf{0 . 1 2}$ & 0.0 & 0.0 & 0.0 & 0.0 & 0.0 & 0.0 & 0.0 & 0.0 & 0.0 \\
Pers & 0.0 & 0.0 & 0.1 & $\mathbf{0 . 1 5}$ & 0.0 & 0.0 & 0.0 & 0.0 & 0.0 & 0.0 & 0.0 & 0.0 \\
FRUp & -0.1 & -0.1 & 0.0 & 0.0 & $\mathbf{0 . 0 2}$ & 0.0 & 0.0 & 0.0 & 0.0 & 0.0 & 0.0 & 0.0 \\
FRUm & -0.2 & -0.2 & 0.1 & 0.2 & 0.0 & $\mathbf{0 . 0 2}$ & 0.0 & 0.0 & 0.0 & 0.0 & 0.0 & 0.0 \\
KVLp & -0.1 & -0.1 & 0.0 & 0.0 & 0.0 & 0.0 & $\mathbf{0 . 0 6}$ & 0.0 & 0.1 & 0.0 & 0.0 & 0.0 \\
KVLm & 0.1 & 0.1 & 0.15 & 0.0 & 0.0 & 0.0 & -0.35 & $\mathbf{0 . 0 3}$ & 0.0 & 0.1 & 0.0 & 0.0 \\
TOTp & 0.0 & 0.0 & 0.0 & 0.0 & 0.0 & 0.0 & 0.7 & 0.0 & $\mathbf{0 . 0 2}$ & 0.0 & 0.0 & 0.0 \\
TOTm & 0.0 & 0.0 & 0.15 & 0.0 & 0.0 & 0.0 & 0.0 & 0.6 & -0.1 & $\mathbf{0 . 0 2}$ & 0.0 & 0.0 \\
ZZ & -0.25 & -0.25 & 0.1 & 0.1 & 0.1 & 0.1 & 0.0 & 0.0 & 0.0 & 0.0 & $\mathbf{0 . 1 2}$ & 0.0 \\
DMG & 0.25 & 0.25 & 0.0 & 0.0 & 0.0 & 0.0 & 0.0 & 0.0 & 0.0 & 0.0 & -0.2 & $\mathbf{0 . 3 5}$ \\
\hline
\end{tabular}

Für die Berechnung des Zuchtziels (Gesamtzuchtwert) werden folgende 12 Merkmale berücksichtigt:

- Fett-kg (Fkg)

- Eiweiß-kg (Ekg)

- Nutzungsdauer (ND)

- Persistenz (Pers)

- Fruchtbarkeit paternal (FRUp)

- Fruchtbarkeit maternal (FRUm)

- Kalbeverlauf paternal (KVLp)

- Kalbeverlauf maternal (KVLm)

- Totgeburt paternal (TOTp)

- Totgeburt maternal (TOTm)

- Zellzahl (ZZ)

- Durchschnittliches Minutengemelk (DMG) 
Wirtschaftlichkeitskoeffizienten der Zuchtwertmerkmale

Zur Berechnung des Indexes müssen zusätzlich die wirtschaftlichen Werte der einzelnen Merkmale mit berücksichtigt werden (Haiger, 2005). Die wirtschaftlichen Gewichte für die Merkmale dieser Arbeit wurden mittels Grenznutzenkalkulationen von Lind (2006) berechnet. Da noch keine Untersuchungen zu wirtschaftlichen Gewichten für ökologische Zuchtmerkmale vorgenommen wurden, handelt es sich hierbei um konventionelle Werte. Aufgrund der Annahme, dass keine großen Unterschiede zwischen den wirtschaftlichen Gewichten beider Wirtschaftsformen vorherrschen, werden diese Gewichte repräsentativ für die ökologischen Zuchtplanungsrechnungen verwendet. In Tabelle 14 sind die wirtschaftlichen Gewichte $(w)$ je genetischer Standardabweichung $\left(\sigma_{A}\right)$ für die verwendeten Merkmale bei der Rasse Deutsche Holstein aufgeführt.

Tabelle 14: Wirtschaftliche Gewichte für Milchleistungs- und funktionale Merkmale (Lind, 2006).

\begin{tabular}{llrrrrr}
\hline Merkmal & Einheit & $\sigma \mathrm{A}$ & $\mathrm{w}$ & $\mathrm{w}^{*} \sigma \mathrm{A}$ & $\sigma \mathrm{w}$ & Gewichtung [\%] \\
\hline Fettmenge & $\mathrm{Kg}$ & 15,6 & $-0,32$ & $-4,98$ & 3,02 & $44 \%$ \\
Eiweißmenge & $\mathrm{Kg}$ & 10,5 & 3,92 & 41,14 & 0,96 & \\
& & & & & & \\
Funktionale ND & Tage (d) & 150 & 0,21 & 31,43 & 0,12 \\
Fruchtbarkeit $\mathrm{p}$ & $\%$ & 5 & 2,44 & 12,21 & 0,05 \\
Fruchtbarkeit $\mathrm{m}$ & $\%$ & 5 & 2,44 & 12,21 & 0,05 & \\
Kalbeverlauf $\mathrm{p}$ & $\mathrm{Klasse}$ & 0,22 & 1,32 & 0,29 & 0,01 & \\
Kalbeverlauf $\mathrm{m}$ & $\mathrm{Klasse}$ & 0,22 & 1,32 & 0,29 & 0,01 & $56 \%$ \\
Totgeburtenrate $\mathrm{p}$ & $\%$ & 2,5 & $-0,22$ & $-0,54$ & 0,01 & \\
Totgeburtenrate $\mathrm{m}$ & $\%$ & 2,5 & $-0,22$ & $-0,54$ & 0,01 & \\
Persistenz & $\mathrm{S}$ & 2,5 & 2,41 & 6,02 & 5,74 & \\
Zellzahlklasse & Klasse & 0,1 & 59,2 & 5,92 & 0,01 & \\
DMG & $\mathrm{kg} /$ min & 0,2 & 12,1 & 2,42 & 0,11 & \\
\hline
\end{tabular}

Die Gewichtung der Merkmalskomplexe ist zu $44 \%$ auf die Leistungsmerkmale und zu $56 \%$ auf die funktionalen Merkmale verteilt. 


\section{Information im Index}

Jede Selektionsgruppe benötigt für die Zuchtwertschätzung Informationen ihrer Ahnen und Nachkommen. Illustrierend für die Vorgehensweise bei der Erhebung der Informationen der Selektionsgruppen im ökologischen Zuchtprogramm sind hier zwei Beispiele aufgeführt. Tabelle 15 zeigt die Berechnungen zur Zuchtwertschätzung (ZWS) von Herdbuchkühen der ökologischen Zuchtstufe und in Tabelle 16 sind entsprechende Berechnungen für die Zuchtwertschätzung von Testbullen der ökologischen Zuchtstufe dargestellt.

Tabelle 15: Informationsquellen für die ZWS der HK-ö (Herdbuchkühe der ökologischen Zuchtstufe), deren Merkmale und Anzahl an wiederholten Messungen.

\begin{tabular}{|c|c|c|c|c|c|c|c|c|c|c|c|c|}
\hline \multirow[b]{2}{*}{ Informationsquelle } & \multicolumn{12}{|c|}{ Merkmale im Index } \\
\hline & $\mathrm{Fkg}$ & Ekg & ND & Pers & FRUp & FRUm & KVLp & KVLm & TOTр & TOTm & zz & DMG \\
\hline Eigenleistung & 1 & 1 & 1 & 1 & & 1 & & 1 & & 1 & 1 & \\
\hline Vater & & & & & 1 & & 1 & & 1 & & & \\
\hline Mutter & 1 & 1 & 1 & 1 & & $3^{*}$ & & $3^{*}$ & & $3^{*}$ & 1 & 1 \\
\hline Vaters Vater & & & & & 1 & & 1 & & 1 & & & \\
\hline Vaters Mutter & 1 & 1 & 1 & 1 & & $3^{*}$ & & $3^{*}$ & & $3^{*}$ & 1 & 1 \\
\hline Mutters Vater & & & & & 1 & & 1 & & 1 & & & \\
\hline Mutters Mutter & 1 & 1 & 1 & 1 & & $3^{*}$ & & $3^{*}$ & & $3^{*}$ & 1 & 1 \\
\hline $\begin{array}{l}\text { Halbschwestern } \\
\text { des Vaters }\end{array}$ & 1 & 1 & 1 & 1 & & $3^{*}$ & & $3^{*}$ & & $3^{*}$ & 1 & 1 \\
\hline $\begin{array}{l}\text { Halbschwestern } \\
\text { der Mutter }\end{array}$ & 1 & 1 & 1 & 1 & & $3^{*}$ & & $3^{*}$ & & $3^{*}$ & 1 & 1 \\
\hline $\begin{array}{l}\text { paternale Halbge- } \\
\text { schwister der } \\
\text { Öko-Herdbuch- } \\
\text { kühe }\end{array}$ & 1 & 1 & 1 & 1 & & $3^{*}$ & & $3^{*}$ & & $3^{*}$ & 1 & 1 \\
\hline
\end{tabular}

In Tabelle 15 sind alle Informationsquellen, die Merkmale und die Anzahl an wiederholten Messungen, die für die Zuchtwertschätzung der Herdbuchkühe der ökologischen Zuchtstufe benötigt werden, entsprechend gekennzeichnet. Die mit einem * (Stern) versehenen Messungen, unterliegen einer Wiederholbarkeit von 0,10. 
Insgesamt tragen jeweils 10 Informationsquellen zum Zuchtwert der Kühe und der Testbullen (Tabelle 16) in der ökologischen Zuchtstufe bei. Bei beiden Gruppen stellen sich die Infoquellen aus der Eigenleistung, der Leistung des Vaters, der Mutter, aus der Leistung des Vaters vom Vater, des Vaters der Mutter, der Mutter des Vaters, der Mutter der Mutter, der Leistung der Halbschwestern des Vaters, der Halbschwestern der Mutter und aus den Leistungen der paternalen Halbgeschwister der Öko-Herdbuchkühe bzw. ÖkoTestbullen zusammen. Zur Beschreibung der einzelnen Informationsquellen und ihrer Bedeutung für die Zuchtwertschätzung der Öko-Herdbuchkühe, werden im Folgenden stellvertretend für alle Informationsquellen nur die Informationen der Eigenleistung, des Vaters und der Mutter näher erläutert.

Aus der ersten Informationsquelle, der Eigenleistung der Herdbuchkühe, geht jeweils eine Messung für das Merkmal Fett-kg, Eiweiß-kg, Nutzungsdauer, Persistenz, Fruchtbarkeit maternal, Kalbeverlauf maternal, Totgeburten maternal und Zellzahl hervor. Der Informationsquelle des Vaters der Kuh wird ebenfalls eine Messung in den Merkmalen Fruchtbarkeit paternal, Kalbeverlauf paternal und Totgeburten paternal entnommen. Bei der Mutter der Kuh werden Fett-kg, Eiweiß-kg, Nutzungsdauer, Persistenz, Zellzahl und die Melkbarkeit (DMG) jeweils einmal gemessen. Die Merkmale Fruchtbarkeit maternal, Kalbeverlauf maternal und Totgeburten maternal unterliegen jedoch jeweils drei Messungen mit der bereits erwähnten Wiederholbarkeit von 0,10.

Die für die Zuchtwertschätzung der Öko-Testbullen benötigten Informationsquellen, deren Merkmale und die jeweilige Anzahl der Messungen sind Tabelle 16 zu entnehmen. Bis auf die Eigenleistung, sind die Informationsquellen identisch mit denen der Öko-Herdbuchkühe und auch die Anzahl der Messungen in den Merkmalen ist die gleiche. Die Informationsquelle Eigenleistung zeigt bei den Testbullen keine Messungen, da davon ausgegangen wird, dass noch keine Informationen vorliegen. Eine weitere Ergänzung benötigen die einmaligen Messungen der Merkmale Kalbeverlauf paternal und Totgeburten paternal der Informationsquelle des Vaters vom Vater. Da bei diesen paternalen Fruchtbarkeitsmerkmalen die Wiederholbarkeit grundsätzlich sehr hoch ist, wird für den Index keine Wiederholbarkeit modelliert. 
Tabelle 16: Informationsquellen für die ZWS der TB-ö (Testbullen der ökologischer Zuchtstufe zum Zeitpunkt der Anpaarung), deren Merkmale und Anzahl an wiederholten Messungen.

\begin{tabular}{|c|c|c|c|c|c|c|c|c|c|c|c|c|}
\hline \multirow[b]{2}{*}{ Informationsquelle } & \multicolumn{12}{|c|}{ Merkmale im Index } \\
\hline & Fkg & Ekg & ND & Pers & FRUp & FRUm & KVLp & KVLm & TOTp & TOTm & $\mathrm{ZZ}$ & DMG \\
\hline \multicolumn{13}{|l|}{ Eigenleistung } \\
\hline Vater & & & & & 1 & & 1 & & 1 & & & \\
\hline Mutter & 1 & 1 & 1 & 1 & & $3^{*}$ & & $3^{*}$ & & $3^{*}$ & 1 & 1 \\
\hline Vaters Vater & & & & & 1 & & 1 & & 1 & & & \\
\hline Vaters Mutter & 1 & 1 & 1 & 1 & & $3^{*}$ & & $3^{*}$ & & $3^{*}$ & 1 & 1 \\
\hline Mutters Vater & & & & & 1 & & 1 & & 1 & & & \\
\hline Mutters Mutter & 1 & 1 & 1 & 1 & & $3^{*}$ & & $3^{*}$ & & $3^{*}$ & 1 & 1 \\
\hline $\begin{array}{l}\text { Halbschwestern } \\
\text { des Vaters }\end{array}$ & 1 & 1 & 1 & 1 & & $3^{*}$ & & $3^{*}$ & & $3^{*}$ & 1 & 1 \\
\hline $\begin{array}{l}\text { Halbschwestern } \\
\text { der Mutter }\end{array}$ & 1 & 1 & 1 & 1 & & $3^{*}$ & & $3^{*}$ & & $3^{*}$ & 1 & 1 \\
\hline $\begin{array}{l}\text { paternale Halbge- } \\
\text { schwister der } \\
\text { Öko-Herdbuch- } \\
\text { kühe }\end{array}$ & 1 & 1 & 1 & 1 & & $3^{*}$ & & $3^{*}$ & & $3^{*}$ & 1 & 1 \\
\hline
\end{tabular}

\subsubsection{Interpretation der Ergebnisse}

Alle alternativen Zuchtprogramme in den Modellrechnungen werden in ZPLAN nach dem naturalen und monetären Zuchtfortschritt, nach dem Züchtungsertrag, den fixen und variablen Züchtungskosten und dem Züchtungsgewinn verglichen und bewertet. Neben diesen Kriterien können Zuchtprogramme auch nach anderen Kriterien, wie z. B. der durchschnittlichen Inzuchtsteigerung in der Population oder der Kapitalrückflussdauer bewertet werden (Gierzinger, 2002). Diese Bewertung verlangt jedoch andere Modellansätze und Computerprogramme und wird daher in diesen Berechnungen nicht durchgeführt.

\subsubsection{Naturaler und monetärer Zuchtfortschritt}

Es wird sowohl der naturale als auch der monetäre Zuchtfortschritt ausgewiesen. Der naturale Zuchtfortschritt beschreibt die durchschnittliche genetische Überlegenheit der Nachkommen der selektierten Tiere einer Selektionsrunde gegenüber den Tieren der Ausgangspopulation. Dabei wird jedes einzelne Merkmal in naturalen Einheiten (z. B. kg 
Milch) pro Generation oder pro Jahr ausgedrückt. Außerdem bezieht sich der naturale Zuchtfortschritt nur auf die Zuchtstufe. Monetärer Zuchtfortschritt ergibt sich aus dem Produkt der naturalen Zuchtfortschritte der einzelnen Merkmale mit ihren jeweiligen aktuellen wirtschaftlichen Gewichten. Die monetären Einheiten beziehen sich auf ein Jahr und gelten ebenso wie der naturale Zuchtfortschritt nur für die Tiere in der Zuchtstufe.

\subsubsection{Der Züchtungsertrag}

Wird der Zuchtfortschritt in monetären Einheiten über die gesamte Investitionsperiode und in der gesamten Population, also sowohl Produktions- als auch Zuchtstufe, betrachtet, spricht man vom Züchtungsertrag. Dieser gibt den diskontierten monetären Ertrag pro Kuh in der Population an, der infolge züchterischer Maßnahmen in der Zuchtstufe für den Zeitraum der Investitionsdauer erwartet werden kann. Die Berechnung erfolgt durch Multiplikation des naturalen Zuchtfortschritts mit dem wirtschaftlichen Gewicht und der standardisierten diskontierten Merkmalsrealisierung, dem SDM-Wert. Der SDM-Wert wird mit folgender Formel ausgedrückt:

$$
S D M_{i j}=\sum_{t=1}^{D} h_{i}^{\prime} m_{j_{t}}\left[\frac{1}{1+r}\right]^{t} .
$$

$h_{i}^{\prime}$ ist der so genannte Realisierungsvektor für das Merkmal $i$. Dieser Vektor standardisiert die Anzahl der Tiere, die ein bestimmtes Merkmal realisieren, auf eine Bezugsbasis, damit die Ergebnisse verschiedener Berechnungen miteinander verglichen werden können. $m_{j_{t}}$ ist der Genanteil-Vektor und beschreibt die Ausbreitung und Verteilung der Gene der Selektionsgruppe $j$ zum Zeitpunkt $t$. Im Gegensatz zu $D$, womit die gesamte Investitionsdauer ausgedrückt wird, bezieht sich $t$ nur auf den Zeitraum vom Bezugspunkt bis zur Merkmalsrealisierung. Der Kalkulationszinssatz wird durch $r$ angegeben.

Da sich die Realisierung der genetischen Überlegenheit in einem Merkmal in einer Population verzögern kann und früher anfallende Merkmalsrealisierungen ökonomisch wertvoller sind als später auftretende Leistungsverbesserungen, ist die Diskontierung erforderlich (Heckenberger, 1991; Kräusslich, 1994;). Der SDM-Wert gibt demnach Informationen darüber von wie vielen Tieren, zu welchem Zeitpunkt und in welchem Ausmaß die genetische Überlegenheit der Selektionsgruppen der Selektionsrunde während der Investitionsdauer realisiert wird. Alle SDM-Werte werden für jede Selektionsgruppe und jedes Merkmal kumulativ für die Dauer der Investitionsperiode berechnet. Sie sind Gewichtungsfaktoren, die die unterschiedlichen Beiträge der Selektionsgruppen zum Züchtungsertrag be- 
rücksichtigen. Der Ertrag bezieht sich nur auf eine Kuh der Gesamtpopulation und ist das Ergebnis nach Ablauf der für das Zuchtprogramm angesetzten Investitionsdauer von 20 Jahren.

\subsubsection{Die Züchtungskosten}

Durch die Erhebung der Züchtungskosten können Aussagen über die Wirtschaftlichkeit von Zuchtprogrammen gemacht werden. Die Höhe des Züchtungsaufwands wird beeinflusst durch Fixkosten und variable Kosten. Fixkosten sind diejenigen Kostenkomponenten, die sich auf die allgemeine Betreuung der Zuchttiere beziehen, z. B. Aufwendungen des Zuchtverbandes für Löhne und Gehälter, Datenerfassung und -verarbeitung, Mietund Reisekosten. Variable Kosten sind die Kosten, die speziell für die Durchführung von Zucht- bzw. spezieller Selektionsmaßnahmen anfallen. Das sind im Einzelnen hauptsächlich die Kosten der Leistungsprüfung, Kosten der Wartehaltung von Bullen und die der Spermagewinnung und Spermalagerung. Produktions- und Lagerungskosten von Spermadosen, die aufgrund ungenügender Zuchtwerte verworfen werden, zählen ebenso zu den variablen Kosten. Alle Züchtungskosten einer Selektionsrunde müssen auf den Bezugszeitpunkt diskontiert werden. Insgesamt werden die Kosten pro Kuh der Gesamtpopulation nach Ablauf der Investitionsperiode ausgegeben.

\subsubsection{Der Züchtungsgewinn}

Der Züchtungsgewinn ist definiert als die Differenz zwischen dem Züchtungsertrag und den fixen und variablen Züchtungskosten. Er bezieht sich ebenfalls auf die gesamte Investitionsperiode und Population und wird pro Kuh ausgewiesen.

\subsubsection{Eigene Berechnungen}

Testbullen und Testanteil

Ein zentraler Bestandteil jedes Zuchtprogramms ist das Testen von Jungbullen (Gierzinger, 2002). Aus diesem Grund wurden im Basiszuchtprogramm unterschiedliche Anzahlen an Testbullen untersucht. Weiterhin wurde innerhalb jeder Testbullengruppe der Testanteil variiert, um ein optimales Verhältnis für das ökologische Zuchtprogramm zu finden. Um den Testanteil möglichst realistisch abzubilden und dem Programm damit entsprechend umsetzbare Werte zu liefern (Willam, 2007), wurde ein Testanteil von 0,1 bis maximal 0,5 gewählt. 
Variation der Fitnessgewichte

In Anlehnung an die Studien von Baumung und Sölkner (1999), in der auf Basis des Gesamtzuchtwertes die Möglichkeiten zur Verwirklichung eines stärker ökologisch orientierten Zuchtziels untersucht werden, wurden dem ökologischen Basiszuchtprogramm vier weitere Varianten mit unterschiedlichen wirtschaftlichen Gewichten in den Fitnessmerkmalen gegenübergestellt. Die Gewichte der Milchmerkmale Fett- und Eiweißmenge wurden dabei gleichzeitig um den jeweils entsprechenden Prozentsatz der veränderten Gewichtung in die entgegengesetzte Richtung verändert.

\section{Veränderung der Populationsgröße}

Für das in Kapitel 3.1 beschriebene ökologische Zuchtprogramm mit einer Populationsgröße von 109.600 Tieren werden in weiteren Berechnungen sechs weitere Populationsgrößen unterstellt. Auch Kalm und Harder (2003) stellten in ihrer Untersuchung dem ökologischen Zuchtprogramm mit einer Populationsgröße von 50.000 Tieren ein konventionelles Zuchtprogramm mit 500.000 Tieren gegenüber. Anders als bei dieser Studie werden die unterschiedlichen Populationsgrößen unter gleichen Bedingungen bzw. in einem ökologisches Zuchtprogramm untersucht.

\section{Töchterleistung pro Anzahl Besamungen}

Um den Informationsgehalt für die Zuchtwertschätzung aus der Nachkommenprüfung zu erhöhen, wird untersucht wie viel Spermaportionen pro Testbulle produziert bzw. verwendet werden, bis die ersten Informationen über dessen Töchterleistungen vorliegen. Die weiblichen Nachkommen der Testbullen liefern die für die Zuchtwertschätzung erforderlichen Daten. Für das Basiszuchtprogramm der ökologischen Kuhpopulation wurden ausgehend vom Basisverhältnis von 1:10 weitere Verhältnisse mit hohen, aber auch niedrigen Wiederfindungsraten angenommen.

\section{Künstliche Besamung}

In Anlehnung an die Grundverordnung von 1991 (Nr. 2092/91/EWG) zum ökologischen Landbau, wonach die Fortpflanzung der Tiere durch Natursprung erfolgen sollte, werden verschiedene Anteile an künstlicher Besamung in der Zucht- und der Produktionsstufe und der komplette Verzicht untersucht. Anhand der Ergebnisse wird der Bedarf des Einsatzes künstlicher Besamung für ein ökologisches Zuchtprogramm diskutiert. 


\section{Ergebnisse}

\subsection{Ergebnisse der Datenauswertung ökologischer Milchviehbetriebe}

\subsubsection{Analyse der genetischen und phänotypischen Trends der Leistungs- und funktionalen Merkmale bei Bullen und Kühen}

In Anlehnung an die Auswertungen nach Stratmann (2006) werden in den nachfolgenden Abbildungen die Ergebnisse der in Kapitel 3.1.1 erläuterten Trenduntersuchungen dargestellt. Auf der x-Achse ist der Betrachtungszeitraum in Geburtsjahren der jeweiligen untersuchten Gruppe angegeben. Die y-Achse zeigt den Wert an, den das Merkmal bzw. der Relativzuchtwert zu dem untersuchten Geburtsjahr einnimmt. Die Einheiten werden merkmalsspezifisch in Kilogramm, Prozent oder Zuchtwertpunkten ausgedrückt.

4.1.1.1 Genetische Trends der Leistungsmerkmale bei Bullen der ökologischen und konventionellen Milchviehzucht

In Abbildung 6 wird der genetische Trend für das Merkmal Milch-kg bei den untersuchten Ökobullen mit dem entsprechenden Trend konventioneller Bullen verglichen. Im Jahr 1993, im ersten Jahr des Betrachtungszeitraums, liegt der Wert mit 182 kg bei den Ökobullen mehr als doppelt so hoch als bei den konventionellen Bullen mit $83 \mathrm{~kg}$. Dieses Verhältnis hebt sich aber im Verlauf der nächsten Jahre auf. Zwischen den Jahren 1995 und 1996 steigt der Wert für konventionelle Bullen von $263 \mathrm{~kg}$ auf $399 \mathrm{~kg}$ an, wohingegen sich bei Ökobullen eine Steigerung von nur 29 kg ergibt. Ab dem Jahr 1997 nähern sich für beide Gruppen die Werte auf ein gleiches Niveau an und erreichen im letzten betrachteten Jahr 973 Milch-kg.

Die Ökobullen steigern somit ihren Wert für Milch-kg von dem Jahr 1993 bis 2000 um etwa das fünffache, die konventionellen Bullen sogar um das elffache. Dies ist insgesamt gesehen ein deutlich positiver Trend beider Gruppen. 


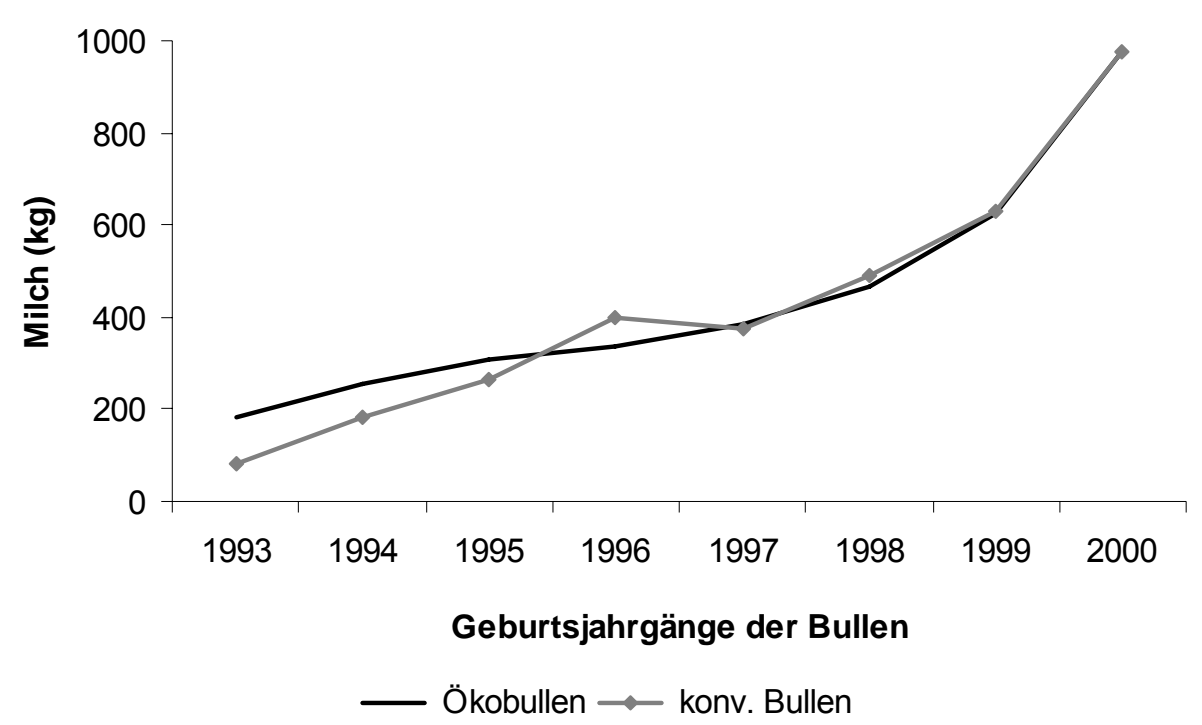

Abbildung 6: Genetischer Trendvergleich der mittleren Zuchtwerte für das Merkmal Milch-kg.

Abbildung 7 zeigt die Entwicklung der Trends im Merkmal Fett-\%. Anders als bei der Milchmenge ist für dieses Merkmal und für beide Gruppen ein leicht negativer Trend zu erkennen. Die Ökobullen zeigen im Jahr 1993 mit + 0,01\% zuerst noch einen positiven Wert, der jedoch bei der weiteren Betrachtung immer stärker in den negativen Wertebereich fällt. In den Jahren 1996 und 1999 steigt die Kurve der Ökobullen minimal an. Bei den konventionellen Bullen ist ab dem Jahr 1996 ein kontinuierlicher Anstieg bis $1999 \mathrm{zu}$ erkennen. Ab dem Jahr 1999 fallen beide Kurven auf mittlere Zuchtwerte von -0,11 bei den Ökobullen und auf $-0,14$ bei den konventionellen Bullen ab.

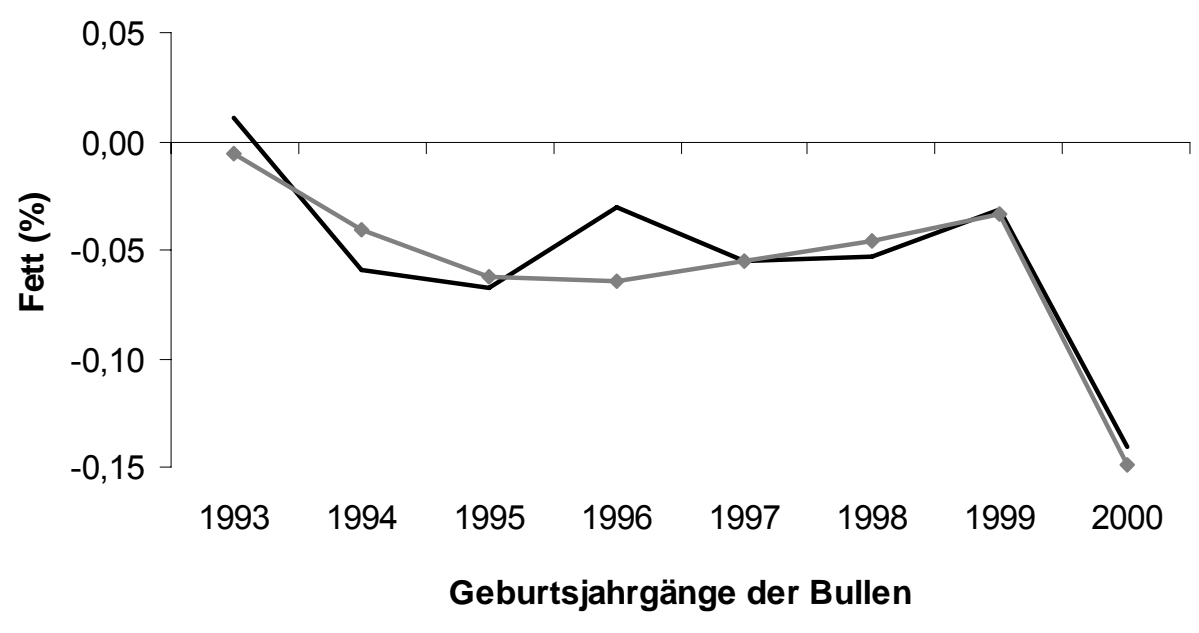

$\longrightarrow$ Ökobullen $\rightarrow$ konv. Bullen

Abbildung 7: Genetischer Trendvergleich der mittleren Zuchtwerte für das Merkmal Fett-\%. 
Für das Merkmal Eiweiß-\% ist die Entwicklung des genetischen Trends bei Öko- und konventionellen Bullen Abbildung $8 \mathrm{zu}$ entnehmen. Alle Werte liegen im negativen Bereich. Der Ausgangswert der Ökobullen liegt hier bei -0,01 und für konventionelle Bullen bei $-0,03$. Die weitere Entwicklung unterliegt keinen starken Schwankungen und verhält sich ähnlich wie beim Merkmal Fett-\%. Neben einem minimalen positiven Anstieg im Jahr 1996, nehmen beide Trends in den Jahren 1997 bis 1999 ein gleiches Niveau ein und sinken schließlich im Jahr 2000 auf Werte von -0,03 bzw. -0,04.

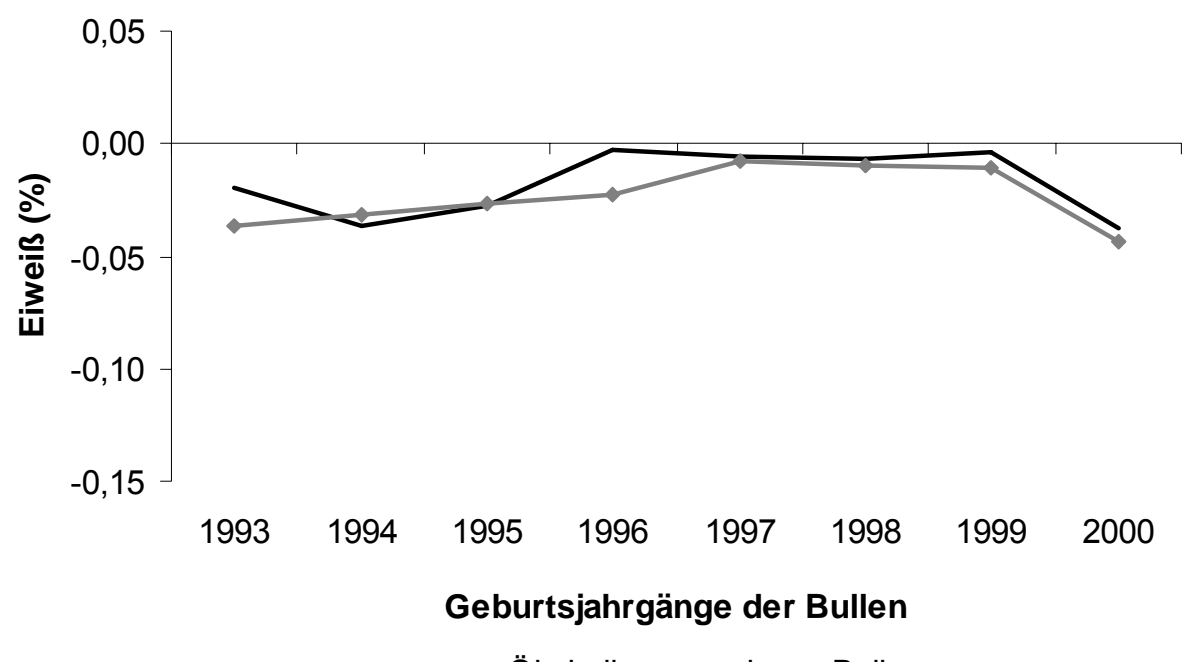

Abbildung 8: Genetischer Trendvergleich der mittleren Zuchtwerte für das Merkmal Eiweiß-\%.

Ein eindeutig positiver Trend ist in der Untersuchung zum Merkmal Fett-kg zu erkennen (Abbildung 9). Nach einem Unterschied im Jahr 1993 zwischen den Gruppen von mehr als $5 \mathrm{~kg}$, passen sich die Werte in den folgenden Jahren wieder an. In den Jahren 1994 bis 1996 steigern sich die Ökobullen um $5 \mathrm{~kg}$ und die konventionellen Bullen um $7 \mathrm{~kg}$. Danach stagnieren die Werte bis 1997. Bis zum Jahr 2000 zeigen beide Bullengruppen einen Anstieg für das Merkmal Fett-kg. 


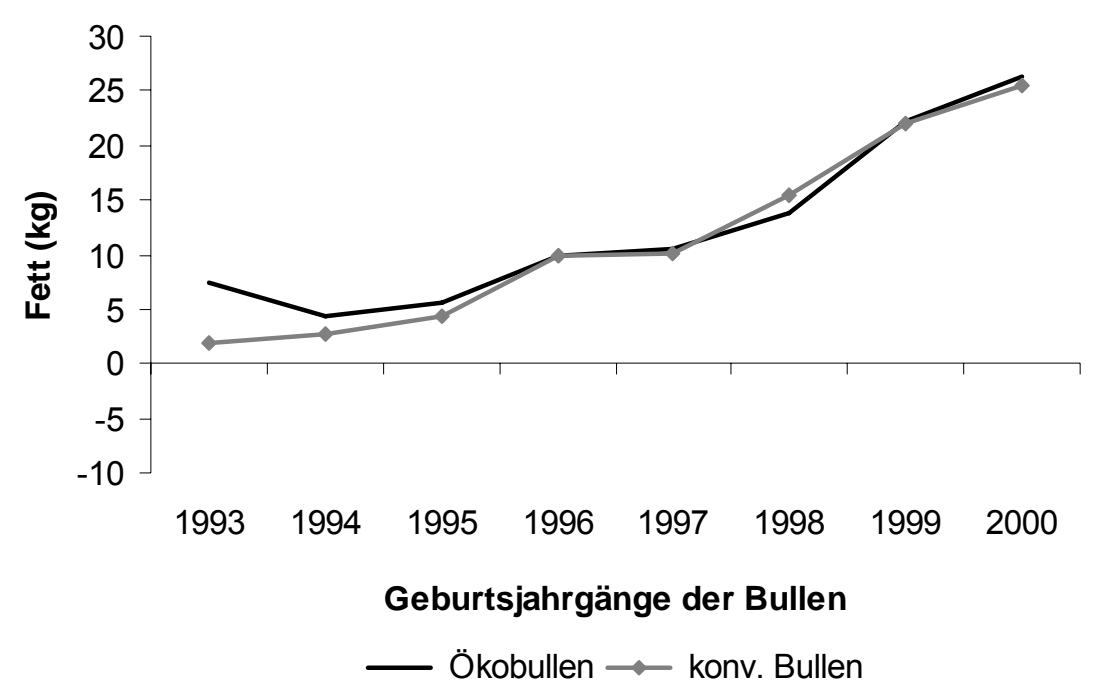

Abbildung 9: Genetischer Trendvergleich der mittleren Zuchtwerte für das Merkmal Fett-kg.

Neben dem nur marginal positiven Trend des Merkmals Eiweiß-\%, ist in Abbildung 10 ein deutlich positiver Trend für das Merkmal Eiweiß-kg zu erkennen.

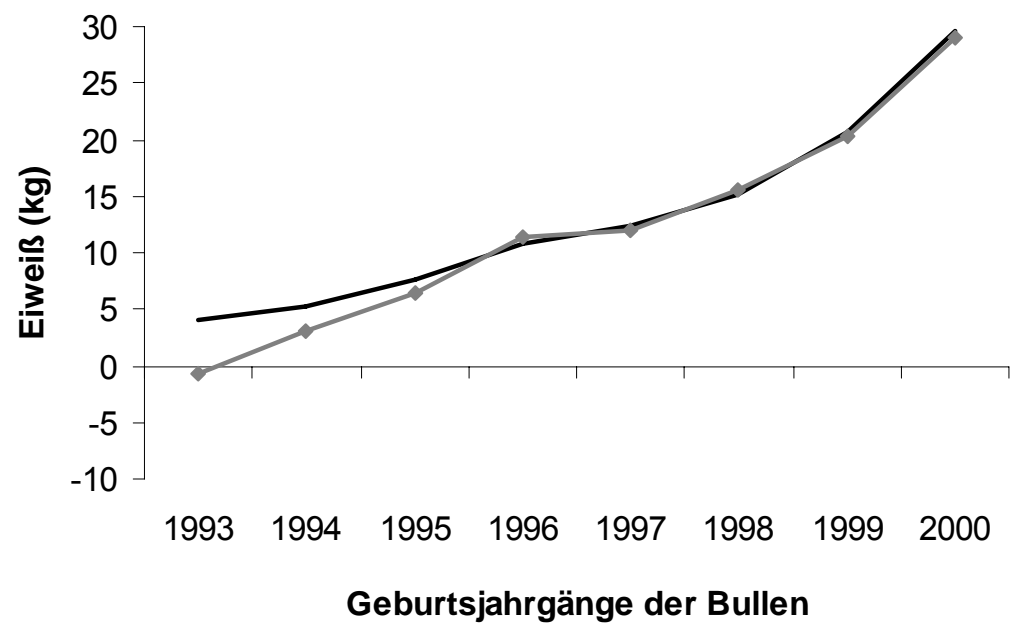

$\longrightarrow$ Ökobullen $\multimap$ konv. Bullen

Abbildung 10: Genetischer Trendvergleich der mittleren Zuchtwerte für das Merkmal Eiweiß-kg.

Die Werte beider Bullengruppen zeigen eine konstante Steigerung. Nach einem zwar schwachen Start der konventionellen Bullen bei -0,6 kg, steigert sich diese Gruppe bis zu einem Wert von $28 \mathrm{~kg}$. Bei den Ökobullen ergibt sich eine positive Steigerung auf bis zu $25 \mathrm{~kg}$. 
4.1.1.2 Genetische Trends in den funktionalen Merkmalen bei Bullen der ökologischen und konventionellen Milchviehzucht

In diesem Kapitel werden die einzelnen Trends der Zuchtleistungsmerkmale Kalbeverlauf, Totgeburten und Non-Return-Rate 90 (NNR 90) anhand ihrer durchschnittlichen Veränderung pro Jahr bewertet (Tabelle 17).

Tabelle 17: Durchschnittliche Veränderung (Regressionskoeffizienten) der Merkmale Kalbeverlauf, Totgeburten und NNR 90.

\begin{tabular}{lcccccc}
\hline & $\begin{array}{c}\text { Kalbeverlauf } \\
\text { paternal }\end{array}$ & $\begin{array}{c}\text { Kalbeverlauf } \\
\text { maternal }\end{array}$ & $\begin{array}{c}\text { Totgeburten } \\
\text { paternal }\end{array}$ & $\begin{array}{c}\text { Totgeburten } \\
\text { maternal }\end{array}$ & $\begin{array}{c}\text { NNR 90 } \\
\text { paternal }\end{array}$ & $\begin{array}{c}\text { NNR 90 } \\
\text { maternal }\end{array}$ \\
\hline Ökobullen & $-0,240$ & $+0,306$ & $+0,186$ & $-0,423$ & $+0,063$ & $-0,057$ \\
konv. Bullen & $-0,110$ & $+0,359$ & $+0,227$ & $-0,412$ & $+0,069$ & $+0,225$ \\
\hline
\end{tabular}

Die Regressionskoeffizienten zum paternalen und maternalen Kalbeverlauf weisen auf unterschiedliche Trends hin. Der paternale Kalbeverlauf ist sowohl bei den konventionellen Bullen, als auch bei den Ökobullen als negativ zu bewerten. Im maternalen Kalbeverlauf sind hingegen positive Entwicklungen in beiden Bullengruppen zu erkennen. Das Merkmal Totgeburten zeigt auf der paternalen Seite ausschließlich positive Trends. Dagegen sind diesmal auf der maternalen Seite negative Regressionskoeffizienten, also negative Trends zu verzeichnen.

Bei Betrachtung des Merkmals Non-Return-Rate 90 zeigen die konventionellen Bullen den deutlich positiveren Trend sowohl auf der paternalen als auch auf der maternalen Seite. Die Ökobullen zeigen nur auf der paternalen Seite eine leichte positive jährliche Entwicklung, auf der maternalen Seite ist der Trend als leicht negativ einzustufen. Die Entwicklung der Trends ist auf die niedrigen Heritabilitäten in den Fitnessmerkmalen zurückzuführen. Aber auch der etwas bessere Trend der konventionellen Bullen kann durch besser angepasste Umweltbedingungen beeinflusst sein.

Tabelle 18: Durchschnittliche Veränderung (Regressionskoeffizienten) der Relativzuchtwerte pro Jahr für Ökobullen im Vergleich zu den konventionellen Bullen.

\begin{tabular}{lcccccc}
\hline & RZG & RZM & RZN & RZE & RZS & RZZ \\
\hline Ökobullen & $+1,970$ & $+2,158$ & $+0,056$ & $+0,619$ & $-0,101$ & $-0,067$ \\
Konventionelle Bullen & $+2,561$ & $+2,677$ & $+0,343$ & $+1,122$ & $-0,131$ & $+0,051$ \\
\hline
\end{tabular}


In Tabelle 18 werden die durchschnittlichen Veränderungen der Relativzuchtwerte RZM, RZN, RZE, RZS, RZZ und des RZG dargestellt. Anhand der Relativzuchtwerte für Milch, Nutzungsdauer und Exterieur, ist bei den konventionellen Bullen gegenüber den Ökobullen ein etwas größerer Zuchtfortschritt zu erkennen. Der Trend im Relativzuchtwert Zuchtleistung, der sich aus den Trends der Merkmale Kalbeverlauf, Totgeburten und NonReturn-Rate ermitteln lässt, zeigt bei den konventionellen Bullen eine leicht positive Entwicklung. Bei den Ökobullen entwickelt sich dieser jedoch leicht negativ. Auch im Merkmal Zellzahl deuten die negativen Regressionskoeffizienten auf einen negativen Trend hin. Da alle Teilzuchtwerte in die Berechnung des Gesamtzuchtwertes mit einfließen, zeigt sich auch hier ein positiver Trend.

\subsubsection{Genetischer Trend bei Ökokühen}

In diesem Kapitel werden die Trends der Milchleistung, Milchinhaltsstoffe, Fett und Eiweiß und der Relativzuchtwerte bzw. des Gesamtzuchtwertes der Ökokühe untersucht. Wie bereits im Methodenteil beschrieben, fängt hier der Betrachtungszeitraum erst im Jahr 1994 an, da für das Jahr 1993 nur die Werte einer Kuh eingetragen sind. Dafür stehen ausreichend Werte jüngere Geburtsjahrgänge wie 2001, 2002 und 2003 zur Verfügung. Abbildung 11 zeigt die durchschnittlichen Zuchtwerte pro Geburtsjahrgang der Ökokühe für das Merkmal Milch-kg, Fett-kg und Eiweiß-kg. Diese Merkmale entwickeln sich eindeutig positiv über den gesamten Betrachtungszeitraum.

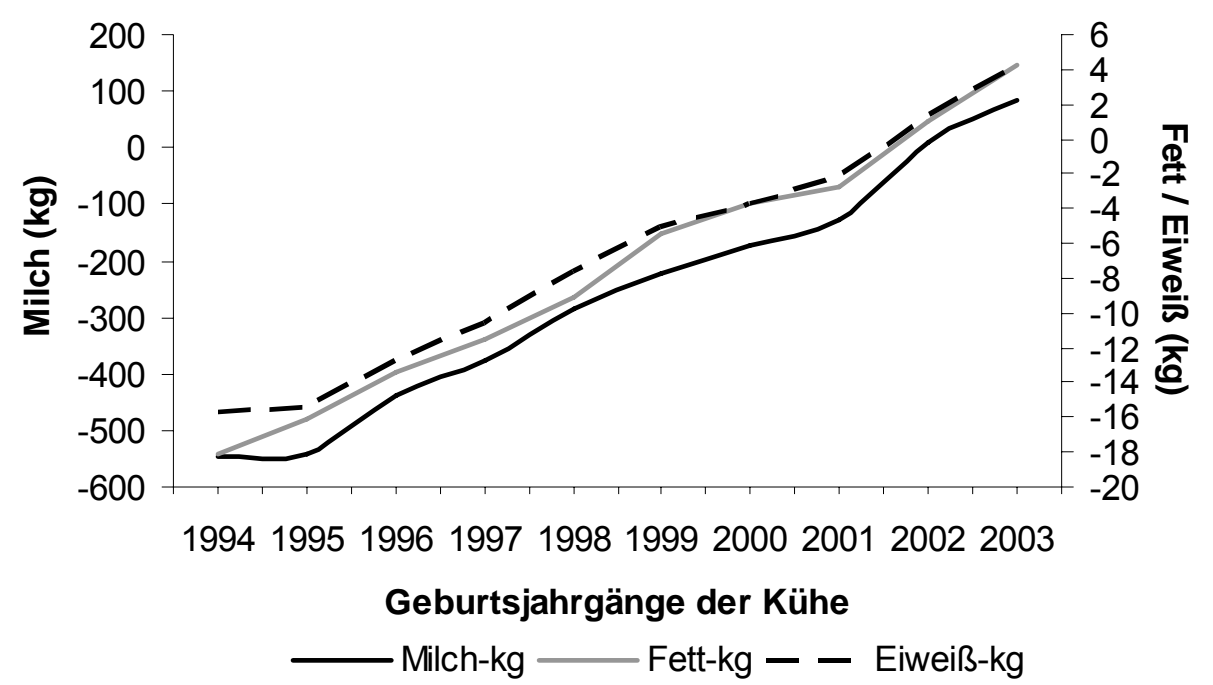

Abbildung 11: Genetischer Trend in den Zuchtwerten Milch-kg, Fett-kg und Eiweiß-kg bei Ökokühen. 
Die stärksten Zunahmen der Milchmenge sind in den Jahren 1996 und 2002. Dort liegen Steigerungen zum Vorjahr in Höhe von $105 \mathrm{~kg}$ und $120 \mathrm{~kg}$ vor. In den anderen Jahren liegt die Zunahme der Milchmenge, ausgenommen 2001 mit 45 kg, bei einem Wert über $50 \mathrm{~kg}$. Schließlich liegt die durchschnittliche jährliche Steigerung des Zuchtwertes Milch$\mathrm{kg}$ bei etwa $+78,16 \mathrm{~kg}$. Die beiden Kurven für Fett-kg und Eiweiß-kg zeigen durchgehend Verbesserungen der Werte und liegen fast auf dem gleichen Niveau. Der Anfangswert für Fett-kg liegt bei -18 und für Eiweiß-kg bei -15. Bis 1999 liegt Eiweiß weiter oberhalb der Fett-kg. Später zeigen die Geburtsjahrgänge 1999 bis 2001 für beide Merkmale identische Werte, die bei $-5,-3$ und $-2 \mathrm{~kg}$ liegen. Die jüngeren Jahrgänge weisen nur noch positive Werte auf. So liegen die Zuchtwert Fett-kg und Eiweiß-kg im Jahr 2002 bei knapp +1 kg und im Jahr 2003 schon bei +4 kg. Für die Merkmale Zuchtwert Fett- $\%$ und Eiweiß- $\%$ sind die genetischen Trends in Abbildung 12 zusammen dargestellt. Die mittleren Zuchtwerte für Fett-\% zeigen nach einem etwas höheren Anfangsniveau bei 0,06 \% einen stärkeren Rückgang als das Merkmal Eiweiß-\%. Die Abnahme beläuft sich auf jährlich etwa $-0,01 \%$. Im Jahr 2002 stehen beide Merkmale auf einem gleichen Level von 0,01\%. Im letzten betrachteten Geburtsjahrgang 2003 liegt der Wert für Fett-\% sogar unter dem des Merkmals Eiweiß-\%.

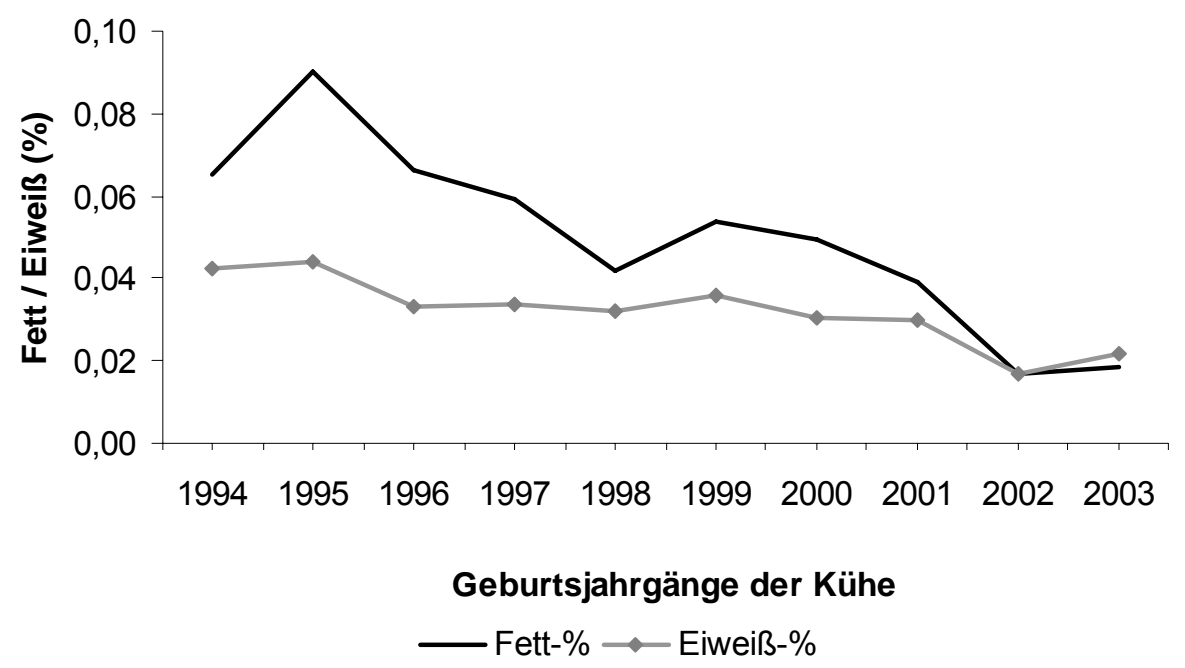

Abbildung 12: Genetischer Trend in den Zuchtwerten Fett- und Eiweiß-\% bei Ökokühen.

Das Merkmal Eiweiß-\% zeigt einen langsameren Rückgang im Verlauf der Jahre. Von einem Ausgangswert der bei 0,04 \% liegt, schwankt das Merkmal in den Jahren 1996 bis 2001 nur minimal und liegt relativ konstant im Wertebereich von 0,03\%. Im Jahr 2002 liegt Eiweiß nur noch bei 0,01\%. Im darauf folgenden Jahr 2003, zeigt sich ein kleiner Anstieg für das Merkmal auf 0,02\%. Diese Entwicklung beider Merkmale über die Jahre ist als negativ zu bewerten. 
Tabelle 19 zeigt die durchschnittlichen Veränderungen der Relativzuchtwerte für Ökokühe. Aufgrund des positiven Trends in der Eiweißmenge zeichnet sich auch im Relativzuchtwert Milchleistung ein positiver Trend ab. Da der Gesamtzuchtwert zum größten Teil durch den Relativzuchtwert Milchleistung bestimmt wird, zeigt sich auch im Gesamtzuchtwert eine positive Entwicklung. Beeinflusst durch die stärkere Gewichtung der funktionalen Merkmale im ökologischen Zuchtziel, entwickeln sich auch die Relativzuchtwerte Exterieur, Nutzungsdauer, Zellzahl und Zuchtleistung positiv innerhalb der betrachteten Jahrgänge.

Tabelle 19: Durchschnittliche Veränderung der Relativzuchtwerte (Regressionskoeffizienten) pro Jahr bei den Ökokühen.

\begin{tabular}{ccccccc}
\hline & RZG & RZM & RZE & RZN & RZS & RZZ \\
\hline Ökokühe & $+1,757$ & $+1,709$ & $+1,478$ & $+0,201$ & $+0,018$ & $+0,079$ \\
\hline
\end{tabular}

\subsubsection{Phänotypischer Trend der Leistungsmerkmale bei Ökokühen}

Im Anschluss an die genetischen Trends werden nun die phänotypischen Trends der Leistungs- und funktionalen Merkmale bei Ökokühen beschrieben. Die Geburtsjahrgänge sind jeweils in Laktationsklassen eingeteilt. Betrachtet werden hierbei jeweils erstlaktierende Kühe, Kühe die in der zweiten Laktation sind und diejenigen, die bereits mehr als zwei Laktationen vorweisen. Die Ergebnisse der phänotypischen Trends in den Leistungsmerkmalen sind in Tabelle 20 in Form von Regressionskoeffizienten dargestellt. Für jede Laktationsgruppe wurden unterschiedliche Geburtsjahrgänge betrachtet.

Tabelle 20: Durchschnittliche Veränderung (Regressionskoeffizienten) pro Jahr für die Einsatzleistung Milch-kg, Fett-kg, Eiweiß-kg, Fett-\% und Eiweiß-\% der Ökokühe in der 1., 2. und > 2. Laktation.

\begin{tabular}{lccccc}
\hline & Milch-kg & Fett-kg & Eiweiß-kg & Fett-\% & Eiweiß-\% \\
\hline Ökokühe, 1. Laktation & $+0,260$ & $+0,006$ & $+0,008$ & $-0,015$ & $+0,000$ \\
Ökokühe, 2. Laktation & $+0,286$ & $+0,010$ & $+0,009$ & $+0,002$ & $+0,002$ \\
$\begin{array}{l}\text { Ökokühe, > 2. Lakta- } \\
\text { tion }\end{array}$ & $+0,348$ & $+0,014$ & $+0,009$ & $+0,002$ & $-0,004$ \\
\hline
\end{tabular}

Die Auswertung der Ökokühe der ersten Laktation bezieht sich auf die Jahre 1997 bis 2003. Für die Untersuchung der Ökokühe in der zweiten Laktation wurde entsprechend ein früherer Betrachtungszeitraum von 1996 bis 2002 gewählt. Bei den Kühen mit mehr 
als zwei Laktationen wurden noch frühere Geburtsjahrgänge von 1995 bis 2001 untersucht. Die höheren Regressionskoeffizienten bei Kühen in höheren Laktationen können physiologisch bedingt sein, da ältere Kühe auch höhere Leistungen durch eine bessere Anpassung des Organismus und einer dadurch bedingten höheren Futterverwertung aufweisen. In allen drei untersuchten Laktationsgruppen sind in Bezug auf die Fett- und Eiweißmenge auch leicht positive Trends zu erkennen. Im Unterschied dazu, ist nur für die Gruppe der Zweitlaktierenden eine positive Entwicklung im Eiweißgehalt zu erkennen. Beim Fettgehalt ist nur bei den zweit- und höher laktierenden Kühen eine positive Tendenz zu erkennen. Die leicht negative Entwicklung im Merkmal Fettgehalt bei Erstlaktierenden ist ebenfalls physiologisch bedingt und auf die Grundfutterleistung zurückzuführen.

\subsubsection{Phänotypischer Trend der funktionalen Merkmale bei Ökokühen}

In diesem Kapitel werden die phänotypischen Trends der funktionalen Merkmale Zellzahl, Zwischenkalbezeit und Erstkalbealter dargestellt. Auch hier wird in drei Laktationsklassen unterschieden. Abbildung 13 zeigt die Entwicklung des Merkmals Zellzahl in verschiedenen Geburtsjahrgängen und den dazugehörenden Laktationsklassen. Auf dem ersten Blick fällt auf, dass die älteren Kühe bzw. die Ökokühe mit mehr als zwei Laktationen in einem deutlich höheren Zellzahlbereich liegen als die Erstlaktierenden. Bei dieser Gruppe zeigt sich der stärkste Anstieg im Jahr 1997. Der Wert erreicht ein Niveau von 367.000 Zellen pro ml. Was in den vorigen Untersuchungen als positive Zunahme gedeutet wurde, ist hier eher schlecht, also als eine negative Entwicklung, anzusehen. Nach diesem stark negativen Trend sinkt der Wert in den darauf folgenden Jahren wieder, bis er schließlich im Jahr 2002 auf das Anfangsniveau von 278.000 Zellen pro ml. abfällt. Aufgrund dieser Entwicklung zeigt diese Gruppe insgesamt einen positiven Trend für das Merkmal Zellzahl. 


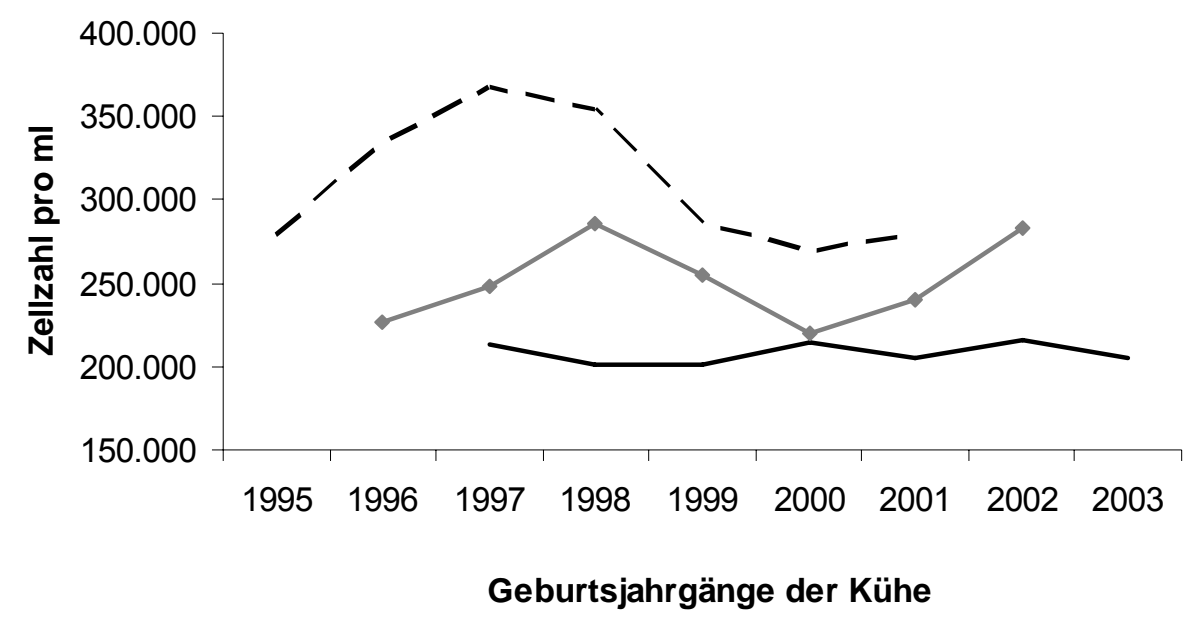

$\longrightarrow$ 1. La. $\longrightarrow$ 2. La. $-\longrightarrow$ 2. La.

Abbildung 13: Phänotypischer Trend für das Merkmal Zellzahl in verschiedenen Laktationen der Ökokühe.

Ebenfalls positiv zeigt sich der Trend in der Gruppe der erstlaktierenden Ökokühe. In dieser Gruppe wird für die betrachteten Jahre durchgehend ein Niveau von 200.000 bis maximal 216.000 Zellen pro $\mathrm{ml}$ gehalten. Im Verlauf der Jahre wechseln sich Abnahme und Anstieg der Zellen bis 2002 ab. Im letzten Jahr 2003 reduziert sich der Zellgehalt auf 204.000. Infolgedessen kann auch hier von einem positiven Trend gesprochen werden.

Die Ökokühe in der zweiten Laktation zeigen im Verlauf der Jahre eine eher negative Entwicklung bzw. einen negativen Trend ihres durchschnittlichen Zellzahlgehaltes. Auf einen Anfangswert von 226.000 Zellen und einen folgenden Anstieg der Zellen auf 285.000, erreicht die Kurve im Jahr 2000 ihr Minimum bei 219.000 Zellen. Dieses Niveau kann sich in den darauf folgenden Jahren nicht halten und steigt wieder bis zu einem Wert von 283.000 Zellen pro $\mathrm{ml}$ an.

Abbildung 14 zeigt die Entwicklung der Zwischenkalbezeit im Verlauf der Jahre und in den zu untersuchenden Laktationsgruppen. Die Laktationsgruppen sind so zu verstehen, dass bei Erstlaktierenden die Zeiten bis zum zweiten Kalb, bei der zweiten Laktationsgruppe die Zeiten vom zweiten bis zum dritten Kalb und bei den Kühen mit mehr als zwei Laktationen alle Zeiten zwischen zwei Kalbungen, die nach mindestens der dritten Kalbung folgen, untersucht werden. 


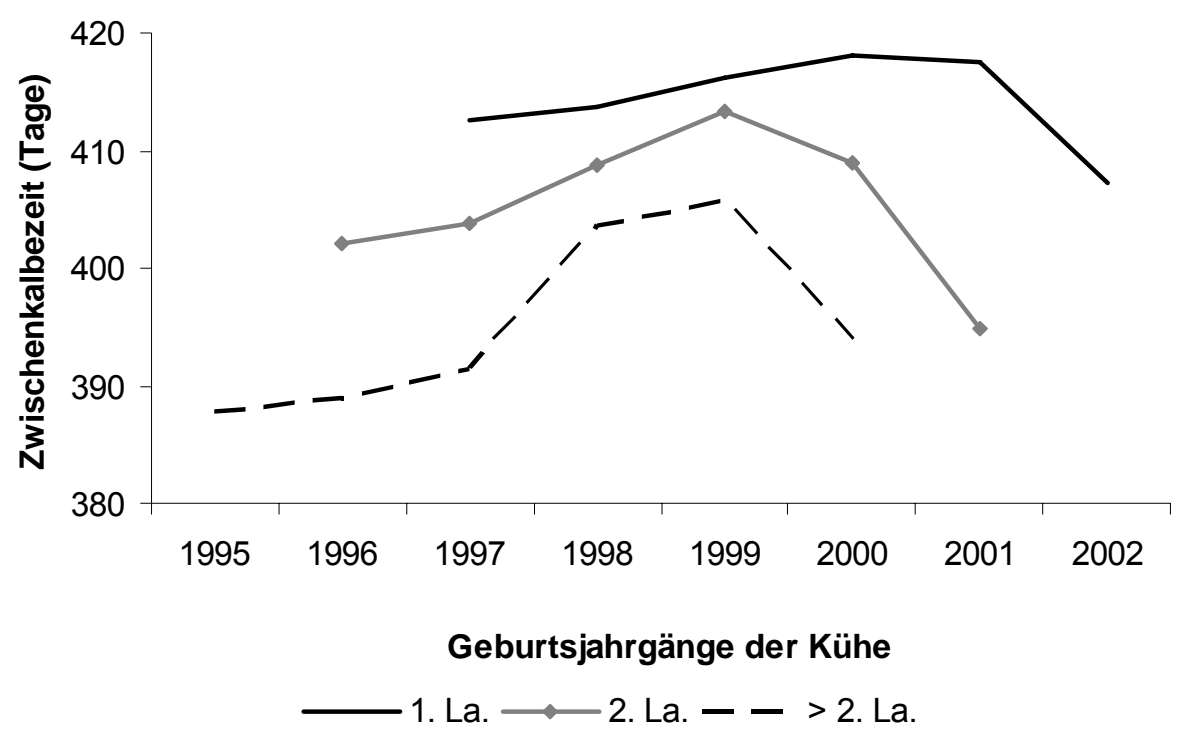

Abbildung 14: Phänotypischer Trend für das Merkmal Zwischenkalbezeit in verschiedenen Laktationen der Ökokühe.

Die durchschnittlichen Zwischenkalbezeiten der erstlaktierenden Kühe liegen in dieser Untersuchung zwischen 407 und 418 Tagen. Der zuletzt genannte Wert wird nach einem leichten Anstieg um jährlich etwa ein bis zwei Tage im Jahr 2000 erreicht. Im folgenden Jahr nimmt der Wert wieder um einen Tag ab und zwei Jahre später, im Jahr 2002, liegt der Wert für die Zwischenkalbezeit nur noch bei 407 Tagen. Das ergibt eine Verringerung von 11 Tagen im Vergleich zum Maximalwert und von fünf Tagen zum Ausgangswert. Trotz einem hohen Niveau zeigt diese Gruppe einen deutlichen positiven Trend für das betrachtete Merkmal.

Die Ökokühe in der zweiten Laktation zeigen ebenfalls einen positiven Trend. Das Niveau dieser Gruppe liegt mit ihrem höchsten Wert von 413 Tagen im Jahr 1999 um fünf Tage niedriger als die Erstlaktierenden. Im Vergleich der Anfangswerte besteht die Differenz bei 10 Tagen und bei den Endwerten liegen die Zweitlaktierenden sogar 13 Tage unter der ersten Gruppe. Das zeigt eindeutig eine positive Entwicklung. Die Kühe der dritten Gruppe mit mehr als zwei Laktationen haben in den Jahren 1995, 1996, 1997 und 2000 die kürzesten Zwischenkalbezeiten dieser Analyse. In den Jahren dazwischen, 1998 und 1999, steigt die Zwischenkalbezeit auf 403 bzw. 405 Tage an, was aber wie bereits für das Jahr 2000 erwähnt, eine Reduzierung um ganze 12 Tage nach sich zieht. Bei Betrachtung der Regressionskoeffizienten ist aber zu erkennen, dass sich eine unerwünschte durchschnittliche Zunahme pro Jahr von 2,91 Tagen ergibt. Alles in allem zeigt dies dann doch eher einen negativen Trend für die Zwischenkalbezeit der dritten Gruppe. 
Die letzte Abbildung dieses Kapitels zeigt den phänotypischen Trend für das Merkmal Erstkalbealter, welches in Monaten ausgedrückt wird (Abbildung 15). Dementsprechend ist auch nur die Kurve für erstlaktierende Kühe der Geburtsjahrgänge 1997 bis 2003 abgebildet. Ausgehend vom Jahr 1997 mit einem Erstkalbealter von etwa 32 Monaten, ist eine stufenweise Abnahme bis zum Jahr 2003 erkennbar. Das im Jahr 2003 festgestellte Erstkalbealter von knapp 27 Monaten nähert sich dem heute gewünschten Erstkalbealter zwischen 24 bis 26 Monaten immer weiter an. Aufgrund dieser Entwicklung und eines Regressionskoeffizienten von -0,48 Monaten, ist dieser Trend als positiv zu interpretieren.

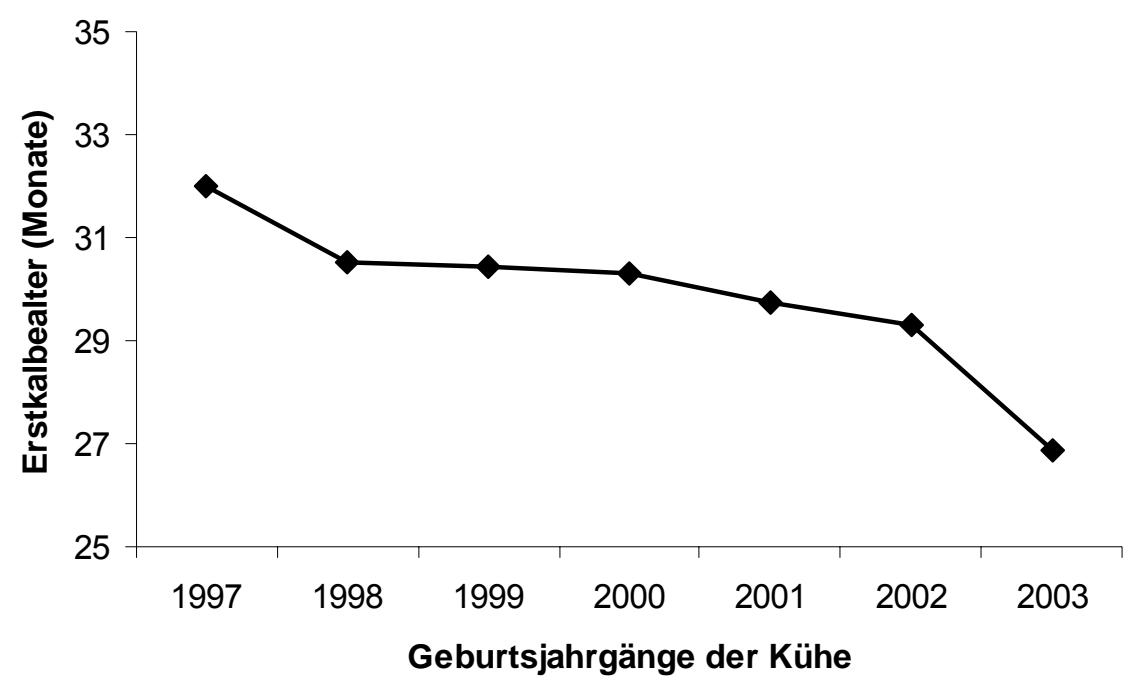

Abbildung 15: Phänotypischer Trend für das Merkmal Erstkalbealter bei erstlaktierenden Ökokühen.

\subsubsection{Genetisches Niveau und ET-Status der eingesetzten Bullen auf ökologischen Milchviehbetrieben in Deutschland}

Die folgenden Abbildungen zeigen verschiedene Ergebnisse zur prozentualen Anzahl der Kühe eines bestimmten Geburtsjahrgangs, deren Väter und Anpaarungsbullen teilweise aus Embryotransfer abstammen. Auf der x-Achse sind die untersuchten Geburtsjahrgänge abgetragen. Die y-Achse zeigt die Anzahl der Kühe eines betrachteten Geburtsjahres, die teilweise einen Vater bzw. Anpaarungsbullen aus Embryotransfer, nachweisen. In den übrigen Abbildungen werden die Kuhväter und Anpaarungsbullen, eingeteilt in ihre Kategorien (Zuchtwertgeprüfter-, Test- oder Deckbulle), gezeigt. An der yAchse wird dabei die prozentuale Anzahl der im jeweiligen betrachteten Jahr geborenen Kühe dargestellt, die dann entweder einen zuchtwertgeprüften Bullen, einen Testbullen oder einen Deckbullen als Vater bzw. Anpaarungsbullen haben. 


\subsubsection{ET-Status der Kuhväter und Anpaarungsbullen}

Das Ergebnis der Untersuchung nach dem ET-Status der Kuhväter aller in Kapitel 3.1.2 beschriebenen Rassen ist in Abbildung 16 dargestellt. Auf dem ersten Blick ist ein symmetrischer Verlauf beider Kurven zu erkennen. Die Anzahl der Kühe, dessen Väter nicht aus Embryotransfer stammen, hat in den letzten Jahren abgenommen. Gleichzeitig werden bei den jüngeren Kühen immer mehr Väter aus Embryotransfer eingesetzt.

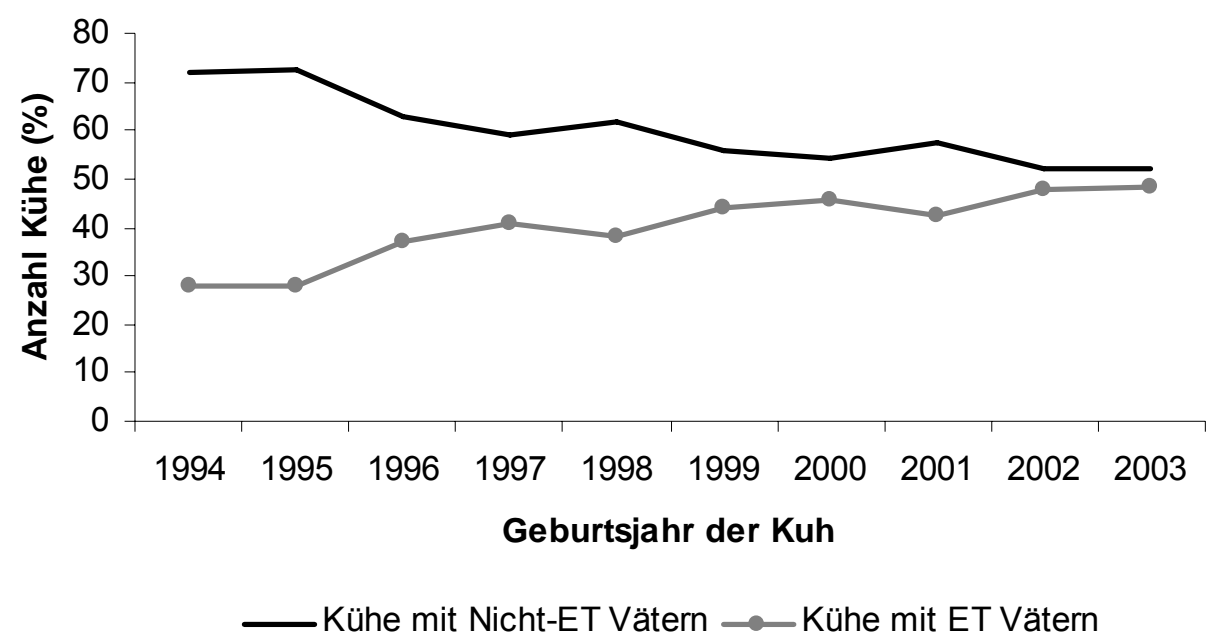

Abbildung 16: ET-Status der Kuhväter bei Betrachtung aller Rassen.

Im Geburtsjahr 1994 haben etwa 72 \% Kühe dieses Jahrgangs einen Vater, der nicht aus Embryotransfer abstammt, und ca. $28 \%$ einen ET-Vater. Dieses Verhältnis ist im Jahr 2003 nicht mehr zu erkennen. Hier sind es nur noch etwa 51 \% der Kühe die keinen ETVater haben, was im Vergleich zum Jahr 1994 einen Rückgang von $20 \%$ ausmacht. Der Anteil der Kühe mit ET-Vätern liegt im Jahr 2003 bei etwa 49 \%. Für den ganzen Zeitraum betrachtet ergibt das eine Zunahme der ET-Bullen von ca. 20 \%. Der ET-Status der Kuhväter ist in Abbildung 17 noch mal speziell für die Rasse Holstein-Friesian untersucht worden. Die Kurvenverläufe sind fast identisch zu denen, der für alle Rassen untersuchten Kuhväter. Das liegt an dem bereits beschrieben hohen Anteil der Rasse HolsteinFriesian von $96 \%$ an allen der Untersuchung zugrunde liegenden Rassen. Dementsprechend verändern sich auch die prozentualen Rückgänge bzw. Zunahmen der Abstammungen der Kuhväter nur minimal. In Anlehnung an die Auswertung für alle Rassen und des zugrunde liegenden hohen HF-Anteils, ergibt sich für die Kühe vom Geburtsjahrgang 1994 bis 2003 ein prozentualer Rückgang der Nicht-ET-Kuhväter von etwa $21 \%$ und ein gleichzeitiger Zuwachs an Kuhvätern, die aus Embryotransfer stammen, von $21 \%$. 


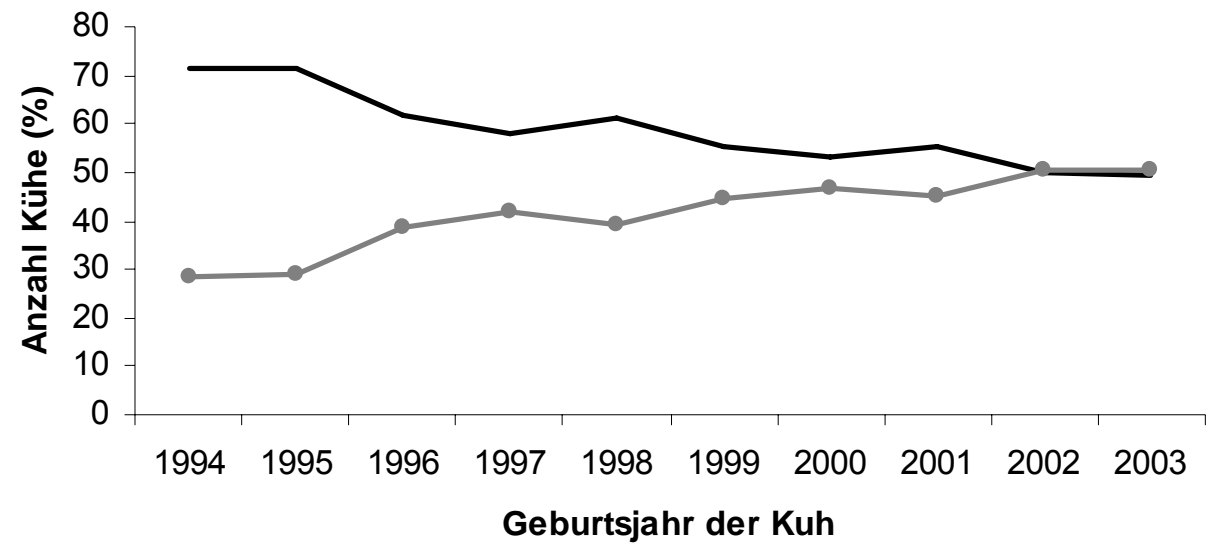

— Kühe mit Nicht-ET Vätern ——Kühe mit ET Vätern

Abbildung 17: ET-Status der Kuhväter bei Betrachtung der Rasse Holstein.

Informationen über den Status der Kuhväter sind der Abbildung 18 und Abbildung 19 zu entnehmen. Diese zeigen den Anteil der Kühe eines Geburtsjahres, dessen Väter entweder zuchtwertgeprüfte Bullen, Test- oder Deckbullen sind. In Abbildung 18 ist das Ergebnis der Auswertung bei allen Rassen dargestellt, in Abbildung 19 speziell nur für HF-Kühe. Auffallend sind in beiden Abbildungen die deutlichen Unterschiede beim Einsatz zuchtwertgeprüfter Bullen und beim Trend zum Deckbulleneinsatz. Die Kühe mit dem Vatertyp Testbulle stellen den kleinsten Anteil dar. Der prozentuale Anteil liegt in allen Geburtsjahren in einem Wertebereich zwischen $3 \%$ und $11 \%$ und entsprechend $5 \%$ bis $15 \%$ bei HF Kühen.

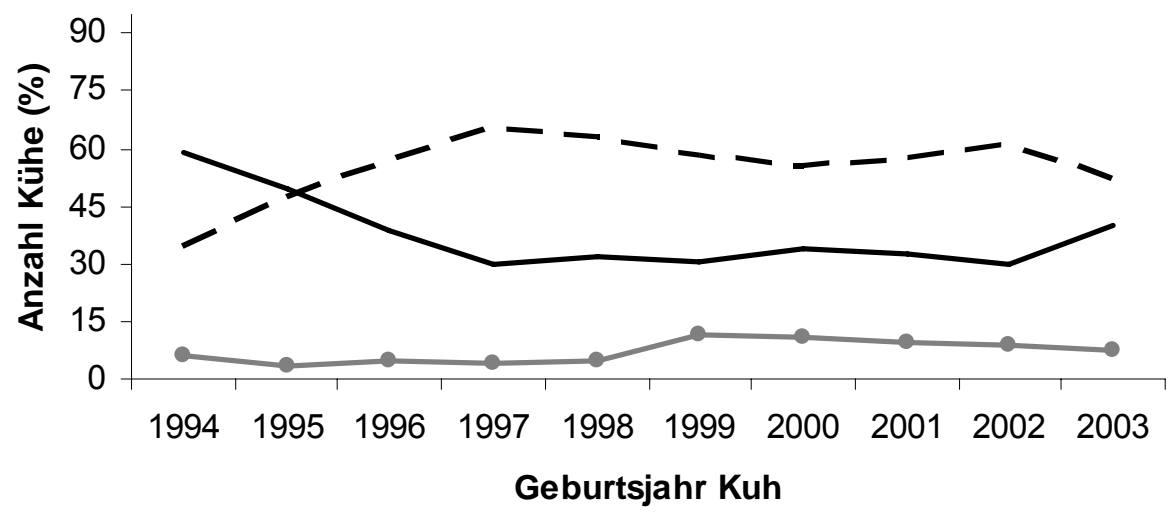

- - Kühe mit geprüften Bullen $\longrightarrow$ Kühe mit Testbullen Kühe mit Deckbullen

Abbildung 18: Status der Kuhväter (Deckbulle/Testbulle/zuchtwertgeprüfter Bulle) bei Betrachtung aller Rassen. 
Bei der Bullenbestimmung in allen Rassen nehmen die Kühe mit einem zuchtwertgeprüften Bullen den größten Anteil ein. Im Verlauf der Geburtsjahre, ist eine Steigerung des Einsatzes dieser Kuhväter von $17 \%$ zu sehen. Der Anteil der Kühe, die Testbullentöchter sind, zeigt nur einen Anstieg von $1 \%$. Deckbullentöchter sind bis zum Geburtsjahrgang 2002 weniger geworden. Für das Jahr 2003 ist jedoch wieder ein Anstieg bei diesem Kuhvatertyp zu erkennen. Insgesamt hat sich der Anteil Kühe, deren Väter Deckbullen sind, für den gesamten Beobachtungszeitraum um $10 \%$ verringert.

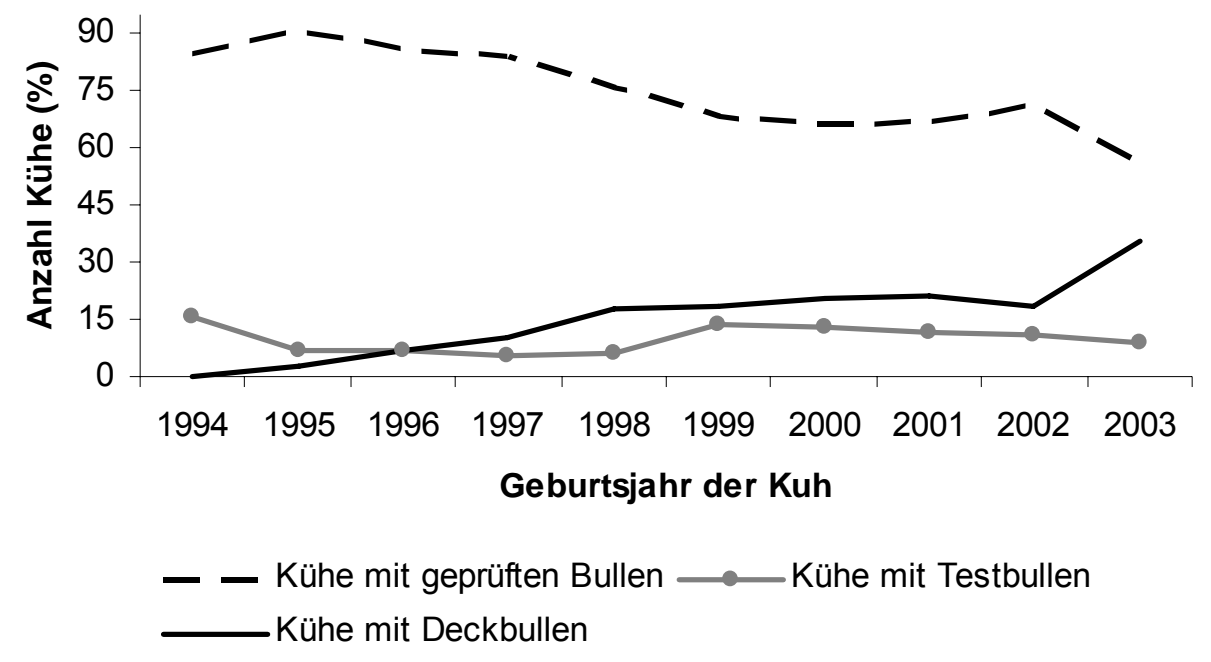

Abbildung 19: Status der Kuhväter (Deckbulle/Testbulle/zuchtwertgeprüfter Bulle) bei Betrachtung der Rasse Holstein.

Bei den Holstein-Friesians unterscheiden sich die Anteile der Kuhvätertypen nicht wesentlich. Auch hier werden überwiegend Kühe, die einen zuchtwertgeprüften Vater haben geboren. Trotzdem ist aber auch zu erkennen, dass mit den jüngeren Geburtsjahrgängen die Häufigkeit solcher Kuhväter um 28 \% zurückgeht. Deutlich wird, dass der Einsatz an Deckbullen als Kuhväter in den letzten Jahren stark an Interesse zugenommen hat. Im Vergleich der Geburtsjahrgänge 1994 bis 2003 ist der Anteil Kühe, die einen Deckbullen zum Vater haben, um $35 \%$ gestiegen. Werden die Anpaarungsbullen der Kühe aller Rassen nach der Abstammung aus Embryotransfer oder nicht untersucht, entwickeln sich die in Abbildung 20 dargestellten Kurven. Diese symmetrische Entwicklung weicht nicht stark von den Ergebnissen der Kuhväter ab. Auch hier ist ein so genannter positiver Trend zum Einsatz von ET-Bullen als Vater des Kalbes deutlich. Ab dem Belegungsjahr 2003 ist der stärkste Anstieg für ET-Bullen zu sehen und eine gleichzeitige Verringerung für Nicht-ETBullen. Die anfängliche Mehrzahl, Kühe mit Nicht-ET-Bullen anzupaaren, nimmt in dem Betrachtungszeitraum um $27 \%$ ab und gleichzeitig werden $27 \%$ mehr Kühe mit ETBullen belegt. 


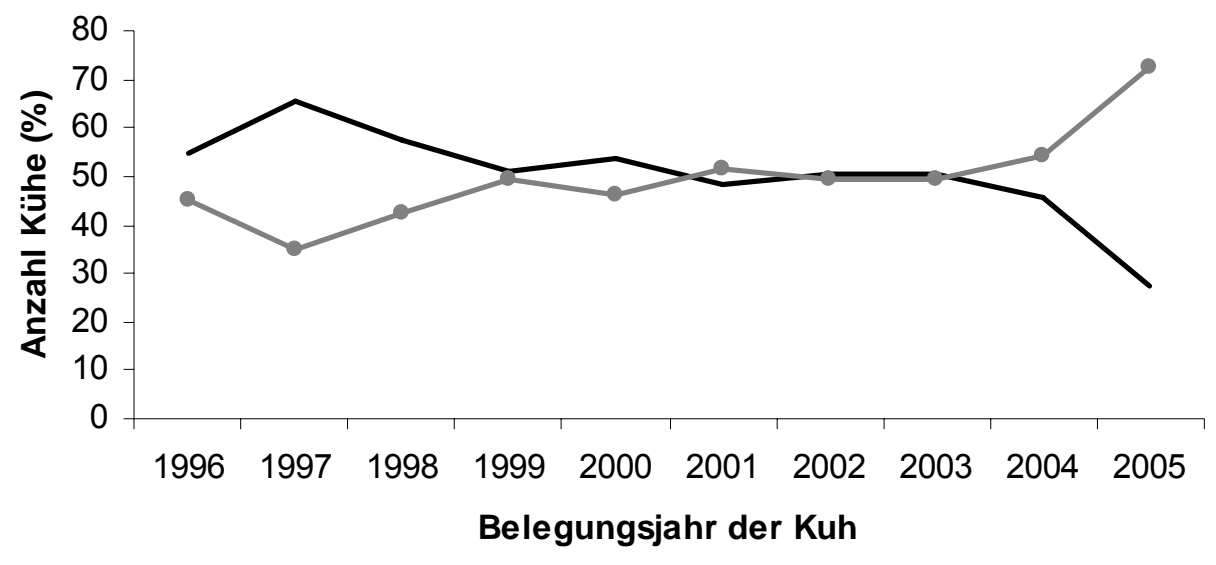

— Kühe mit Nicht-ET-Bullen —-Kühe mit ET-Bullen

Abbildung 20: ET-Status des Anpaarungsbullen bei Betrachtung aller Kuhrassen.

Bei der in Abbildung 21 gezeigten Analyse nach dem Typ der oben untersuchten Anpaarungsbullen sind einige Unterschiede zu den Kuhvätern zu erkennen. Der Anteil Kühe die mit Deckbullen angepaart werden, nimmt zwar im Verlauf der Belegungsjahre um 8 \% zu, aber in dieser Untersuchung stellt diese Gruppe den geringsten Anteil dar. Die Kühe mit zuchtwertgeprüften Bullen zu belegen, dominiert vor dem Einsatz eines Testbullen. Diese zeigen aber in den jüngeren Belegungsjahren ab 2004 einen deutlichen Anstieg.

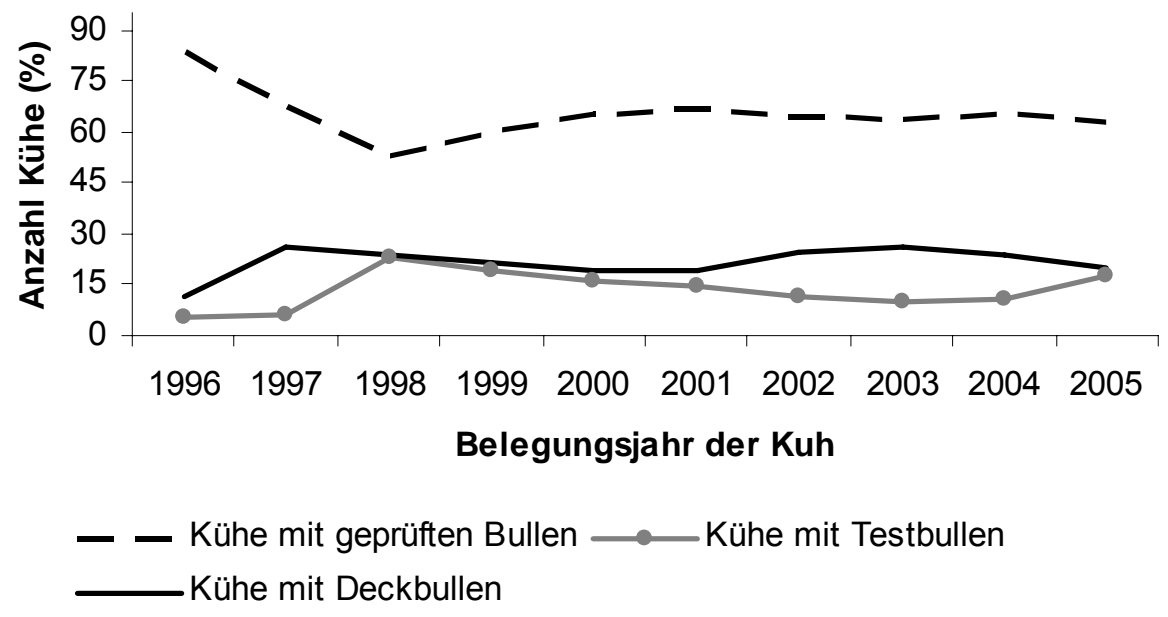

Abbildung 21: Status der Anpaarungsbullen (Deckbulle/Testbulle/zuchtwertgeprüfter Bulle) bei Betrachtung aller Rassen.

Nach einem starken Abfall der Häufigkeit der Kühe, die mit geprüften Bullen angepaart werden, im Jahr 1998 geht der Einsatz auch insgesamt um 20 \% zurück. Der Einsatz von Testbullen zur Belegung der Kühe zeigt insgesamt eine positive Entwicklung von 12 \%. 


\subsubsection{Genetische Trends der Kuhväter}

Abbildung 22 zeigt einen zusammenfassenden Überblick über die genetischen Trends aller Relativzuchtwerte für das Jahr 2000 und beide Kuhvätergruppen. Bei dieser Gegenüberstellung wird deutlich, dass die ET-Kuhväter im Gesamtzuchtwert und den Teilzuchtwerten RZN, RZZ, RZE und RZM höhere Zuchtwertpunkte haben als die Nicht-ETKuhväter. Diese liegen für das betrachtete Jahr nur mit dem Teilzuchtwert RZS über dem Wert der ET-Kuhväter.

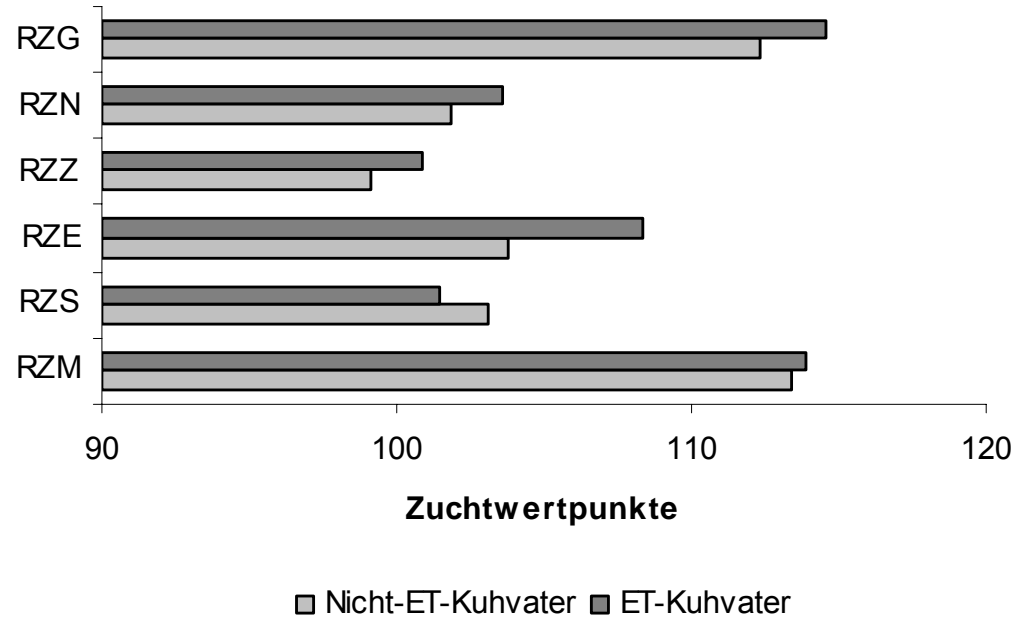

Abbildung 22: Trendvergleich zwischen Nicht-ET - und ET-Kuhvätern des Geburtsjahres 2000.

Zusammenfassend ist zu sagen, dass es keine großen Unterschiede bei Betrachtung der genetischen Niveaus der Nicht-ET- und ET-Kuhväter zu nennen gibt. In den betrachteten Jahren sind jedoch die ET-Kuhväter minimal besser. Ergänzend dazu zeigen die Relativzuchtwerte RZN, RZS und RZE die deutlichsten Spannen zwischen den Vergleichsgruppen von mindestens zwei bis zu vier Zuchtwertpunkten.

\subsection{Zuchtplanung}

In Anlehnung an das in Kapitel 3.2 unter den Rahmenbedingungen der ökologischen Milchviehhaltung modellierte Zuchtprogramm werden in den folgenden Abschnitten die Ergebnisse der Zuchtplanungsrechnungen dargestellt und nach ihren Erfolgsparametern quantifiziert. 


\subsubsection{Interpretation der Ergebnisse aus dem Basislauf}

Nachdem für das zu untersuchende ökologische Zuchtprogramm ein Basislauf anhand der in vorigen Kapiteln beschriebenen Inputparameter berechnet wurde, erfolgte die erste Analyse des naturalen und monetären Zuchtfortschrittes, des Züchtungsertrages, der Züchtungskosten und des Züchtungsgewinns. Tabelle 21 und Tabelle 22 zeigen eine Übersicht zu den wichtigsten Ergebnisse und deren Bezugseinheiten.

Tabelle 21: Monetärer Zuchtfortschritt pro Jahr gesamt $(€)$, Züchtungsertrag (€), Züchtungskosten $(€)$ und Züchtungsgewinn $(€)$ nach den ersten Berechnungen für das Zuchtprogramm der Basispopulation und einer Investitionsperiode von 20 Jahren.

\begin{tabular}{lc}
\hline Parameter und Zeiteinheit & Summe $(€)$ \\
\hline $\begin{array}{l}\text { monetärer Zuchtfortschritt der Zuchtstufe pro Jahr innerhalb } \\
\text { der Investitionsperiode }\end{array}$ & 10,33 \\
Züchtungsertrag pro Kuh nach 20 Jahren & 46,76 \\
Züchtungskosten pro Kuh nach 20 Jahren & 29,33 \\
Züchtungsgewinn pro Kuh nach 20 Jahren & 17,42 \\
\hline
\end{tabular}

Wie bereits in Kapitel 3.2.4 beschrieben, werden die Ergebnisse für verschiedene Tiergruppen und unterschiedliche Zeiteinheiten ausgegeben. So bezieht sich der monetäre Zuchtfortschritt ausschließlich auf die Kühe in der Zuchtstufe und nicht auf die gesamte Population des Zuchtprogramms und wird für ein Jahr angegeben. Anders als beim Zuchtfortschritt errechnen sich der Züchtungsertrag, die Züchtungskosten und der Züchtungsgewinn über die gesamte Investitionsperiode und beziehen sich auf die Kühe der Zuchtund Produktionsstufe. Für den Basislauf heißt das, dass nach 20 Jahren pro Kuh 46,76€ Züchtungsertrag erreicht wird. Die Kosten, die für das Zuchtprogramm pro Kuh in den 20 Jahren entstehen liegen bei 29,33€ und der Züchtungsgewinn, der pro Kuh nach Ablauf der Investitionsperiode erzielt wird, liegt bei 17,42 €. Aufgerechnet auf die Gesamtpopulation kann demnach mit diesem Zuchtprogramm ein Züchtungsgewinn von schätzungsweise 1.900.000€ erreicht werden. Alle Angaben zum Züchtungsertrag, den Züchtungskosten und dem Züchtungsgewinn sind auf das Anfangsjahr diskontiert.

In Tabelle 22 sind der naturale und der monetäre Zuchtfortschritt der einzelnen Merkmale pro Jahr dargestellt. Um eine bessere Vergleichbarkeit zwischen Merkmalen zu ermöglichen, wird der naturale Zuchtfortschritt auch standardisiert in Prozent einer genetischen Standardabweichung des jeweiligen Merkmals ausgedrückt. Dadurch können die einzel- 
nen Merkmale im Gesamtzuchtwert direkt miteinander verglichen und anschaulicher dargestellt werden. Zur Berechnung der einzelnen monetären Zuchtfortschritte wird der naturale Zuchtfortschritt der entsprechenden Merkmale mit dem dazugehörigen wirtschaftlichen Gewicht multipliziert.

Tabelle 22: Naturaler und monetärer Zuchtfortschritt der Merkmale pro Jahr (standardisierter nat ZF/Jahr (in $\mathrm{s}_{\mathrm{A}}{ }^{*} 100$ ), in \% bzw. mon ZF/Jahr $(€)$, absoluter und relativer Anteil der Merkmalsgruppen Milch und Fitness am gesamten monetären Zuchtfortschritt pro Jahr (monZF/Jahr gesamt, $€$ ) für die Basispopulation.

\begin{tabular}{|c|c|c|c|c|c|c|c|}
\hline Merkmal & & & $\begin{array}{l}\text { naturaler } \\
\text { ZF pro }\end{array}$ & $\begin{array}{l}\text { naturaler } \\
\text { ZF/Jahr } \\
(\mathrm{sA} * 100)\end{array}$ & $\begin{array}{l}\text { monetärer } \\
\text { ZF/Jahr }(€)\end{array}$ & $\begin{array}{l}\text { rel. Anteil } \\
\text { der Merk- }\end{array}$ & $\begin{array}{l}\text { rel. Anteil } \\
\text { Merkmals- }\end{array}$ \\
\hline Milch & Fkg & $\mathrm{kg}$ & 0,1358 & 13,58 & $-0,6762$ & $-6,54$ & 63,08 \\
\hline & Ekg & $\mathrm{kg}$ & 0,1748 & 17,48 & 7,1912 & 69,61 & \\
\hline Fitness & ND & Tage & 0,0974 & 9,74 & 3,0612 & 29,63 & \\
\hline & Pers & $S_{A}$ & 0,0646 & 6,46 & 0,3888 & 3,76 & \\
\hline & FRUp & $\%$ & $-0,0085$ & $-0,85$ & $-0,1037$ & $-1,00$ & \\
\hline & FRUm & $\%$ & 0,0262 & 2,62 & 0,3199 & 3,09 & \\
\hline & KVLp & Klasse & $-0,0232$ & $-2,32$ & $-0,0067$ & $-0,06$ & \\
\hline & KVLm & Klasse & 0,0488 & 4,88 & 0,0141 & 0,13 & \\
\hline & TOTр & $\%$ & $-0,0004$ & $-0,04$ & $-0,0002$ & 0,00 & \\
\hline & TOTm & $\%$ & 0,0263 & 2,63 & 0,0142 & 0,13 & \\
\hline & ZZ & Klasse & 0,0014 & 0,14 & 0,0082 & 0,07 & \\
\hline & DMG & $\mathrm{kg} / \mathrm{min}$ & 0,0485 & 4,85 & 0,1173 & 1,13 & \\
\hline monetäre & ZF/Jahr & esamt & & & $10,33 €$ & $100 \%$ & $100 \%$ \\
\hline
\end{tabular}

Wie auch schon in Tabelle 21 gezeigt, ergibt sich nach dem ersten Rechendurchlauf ein monetärer Zuchtfortschritt pro Jahr in Höhe von etwa 10,33€. Den höchsten Anteil am gesamten monetären Zuchtfortschritt zeigt das Merkmal Eiweißmenge mit fast 70 \%. Den zweithöchsten Anteil mit knapp 30 \% nimmt das aus ökologischer Sicht züchterisch interessantere Merkmal Nutzungsdauer ein. Weitere wichtige funktionale Merkmale wie die Persistenz und die maternale Fruchtbarkeit zeigen mit jeweils $3 \%$ die dritthöchsten relativen Anteile am gesamten monetären Zuchtfortschritt. Da züchterisch eine niedrige Zellzahl gewünscht ist und für diese Berechnung ein positives wirtschaftliches Gewicht einge- 
geben wurde, ist der zwar geringe relative Anteil am monetären Zuchtfortschritt als positiv zu bewerten.

Die relativen Anteile der Merkmalsgruppen am gesamten monetären Zuchtfortschritt teilen sich in 63,08 \% für den Bereich Milch und 36,92 \% für den Komplex Fitness auf. Diese Werte zeigen sehr deutlich, dass die Milchmerkmale Fett- und Eiweißkilogramm den monetären Zuchtfortschritt am stärksten beeinflussen, obwohl die Fitnessmerkmale insgesamt wirtschaftlich höher gewichtet werden (vgl. Tabelle 14). Ursachen für diese Entwicklung sind die höheren Heritabilitäten in den Merkmale Fett- und Eiweißmenge und die hohen Korrelationen dieser Merkmale zueinander. Da die Heritabilitäten der Fitnessmerkmale geringer sind und auch die Zuverlässigkeit der Zuchtwertschätzung für die Fitnessmerkmale gering ist, fällt entsprechend der Anteil dieser Merkmalsgruppe am gesamten monetären Zuchtfortschritt gering aus.

\subsubsection{Variation im Testanteil und der Anzahl an Testbullen}

Die Höhe des Testanteils ist bedeutend für den Zuchtfortschritt in der Population. Durch eine Erhöhung des Testanteils wird bei gleicher Anzahl Testbullen automatisch ein Anstieg der Töchterzahl pro Testbulle erreicht. Dieses führt schließlich zur Steigerung der Genauigkeit der Zuchtwertschätzung. Allerdings wird damit auch die Anzahl Kühe reduziert, die an Wiedereinsatzbullen angepaart werden können. In diesem Kapitel werden die Ergebnisse bei variiertem Testanteil, variierter Anzahl an Testbullen und schließlich die Entwicklung bei gleichzeitiger Variation beider Parameter dargestellt.

In der folgenden Tabelle 23 sind zunächst der naturale Zuchtfortschritt pro Merkmal (multipliziert mit 100) und der gesamte monetäre Zuchtfortschritt bei Variation des Testanteils abgebildet. Ausgehend von einem Testanteil von 0,25 wird dieser schrittweise auf 0,1 verringert bzw. auch bis auf 0,5 erhöht. Höhere Testanteile werden nicht weiter untersucht, da auch Gierzinger (2002) einen Testanteil ab $40 \%$ als unrealistisch bezeichnet und hohe Testanteile für das Computerprogramm ZPLAN nicht mehr umsetzbar sind (Willam, 2007). Allen folgenden Ergebnissen wird ein Basisszenario mit 20 Testbullen und einem Testanteil von $25 \%$ unterstellt. 
Tabelle 23: Einfluss unterschiedlicher Testanteile auf den naturalen Zuchtfortschritt (in $\mathrm{S}_{\mathrm{A}}$ * 100) und den gesamten monetären Zuchtfortschritt pro Jahr ( $€$ ) im Vergleich zum Basisszenario (fett gedruckt).

\begin{tabular}{lccccccc}
\hline Merkmal & Einheit & 0,1 & 0,2 & $\mathbf{0 , 2 5}$ & 0,3 & 0,4 & 0,5 \\
\hline Fkg & kg & 13,37 & 13,51 & $\mathbf{1 3 , 5 8}$ & 13,64 & 13,78 & 13,93 \\
Ekg & $\mathrm{kg}$ & 17,18 & 17,39 & $\mathbf{1 7 , 4 8}$ & 17,56 & 17,74 & 17,93 \\
& & & & & & & \\
ND & Tage & 8,98 & 9,55 & $\mathbf{9 , 7 4}$ & 9,90 & 10,18 & 10,41 \\
Pers & $\mathrm{S}_{\mathrm{A}}$ & 6,28 & 6,42 & $\mathbf{6 , 4 6}$ & 6,50 & 6,58 & 6,65 \\
FRUp & $\%$ & $-0,82$ & $-0,84$ & $\mathbf{- 0 , 8 5}$ & $-0,86$ & $-0,88$ & $-0,91$ \\
FRUm & $\%$ & 2,29 & 2,53 & $\mathbf{2 , 6 2}$ & 2,70 & 2,83 & 2,95 \\
KVLp & Klasse & $-2,27$ & $-2,30$ & $\mathbf{- 2 , 3 2}$ & $-2,33$ & $-2,35$ & $-2,37$ \\
KVLm & Klasse & 4,73 & 4,84 & $\mathbf{4 , 8 8}$ & 4,92 & 4,98 & 5,04 \\
TOTp & $\%$ & $-0,04$ & $-0,04$ & $\mathbf{- 0 , 0 4}$ & $-0,04$ & $-0,04$ & $-0,04$ \\
TOTm & $\%$ & 2,52 & 2,60 & $\mathbf{2 , 6 3}$ & 2,65 & 2,69 & 2,73 \\
ZZ & Klasse & 0,01 & 0,11 & $\mathbf{0 , 1 4}$ & 0,16 & 0,19 & 0,20 \\
DMG & kg/min & 4,77 & 4,83 & $\mathbf{4 , 8 5}$ & 4,88 & 4,92 & 4,97 \\
\hline mon ZF/Jahr & & 9,91 & 10,21 & $\mathbf{1 0 , 3 3}$ & 10,42 & 10,59 & 10,75 \\
gesamt $(€)$ & & & & & & & \\
\hline
\end{tabular}

Die Ergebnisse zeigen deutlich, dass sowohl der naturale als auch der monetäre Zuchtfortschritt bei einer kontinuierlichen Erhöhung des Testanteils ansteigen. Im monetären Zuchtfortschritt sind die Unterschiede ab einem Testanteil von 0,2 jedoch gering. Vergleichen zum Basiswert bei 10,33€ steigt der monetäre Zuchtfortschritt bei einem Testanteil von 0,5 um nur 0,42€ an. Eine Verringerung des Testanteils bewirkt gleichzeitig einen Rückgang im naturalen und monetären Zuchtfortschritt. Der monetäre Zuchtfortschritt nimmt bei einem Testanteil von 0,1 im Vergleich zum Basisszenario mit $25 \%$ Testanteil um 0,42€ ab. Der Anstieg des monetären Zuchtfortschritts wird durch das kürzere Generationsintervall bei verstärktem Jungbulleneinsatz bewirkt. Für die Abnahme des monetären Zuchtfortschritts durch Verringerung der Testkapazität sind die ungenau geschätzten Zuchtwerte der Testbullen im Vergleich zu Altbullen verantwortlich. 
In Abbildung 23 ist deutlich zu sehen, dass sich der naturale Zuchtfortschritt am stärksten im Merkmal Zellzahl verändert wenn der Testanteil von $25 \%$ auf 50\% erhöht wird. Aber auch die funktionalen Merkmale maternale Fruchtbarkeit und Nutzungsdauer zeigen deutlich positive Veränderungen. Trotz der niedrigen Heritabilitäten in den funktionalen Merkmalen konnte aufgrund des hohen Testumfangs mehr Töchterinformationen pro Testbulle gewonnen werden und somit die Genauigkeit der Zuchtwertschätzung verbessert werden. Dadurch wurde ein höherer Zuchtfortschritt in diesen Merkmalen realisiert. Eine Verschlechterung der naturalen Zuchtfortschritte ist ausschließlich bei den paternalen Merkmalen zu erkennen.

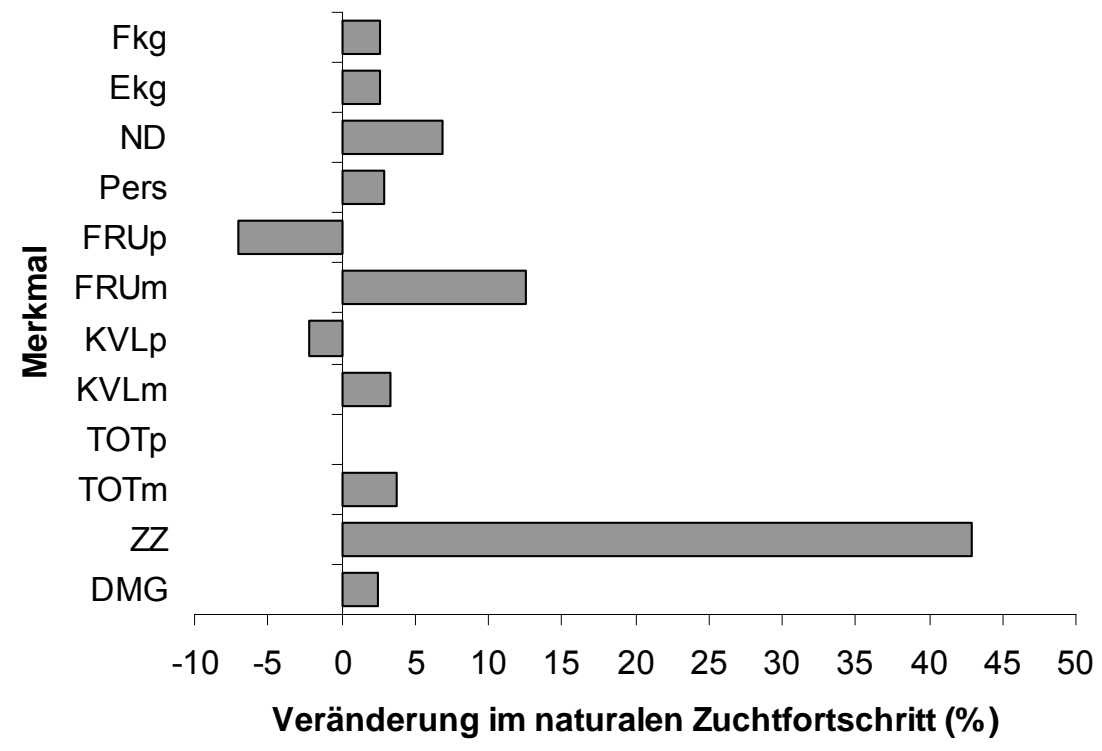

Abbildung 23: Prozentuale Veränderung der naturalen Zuchtfortschritte der Merkmale bei Veränderung des Testanteils von $25 \%$ auf $50 \%$.

Ergänzend zu den in Tabelle 23 dargestellten Ergebnissen bei Variation der Testanteile im Basiszuchtprogramm zeigt Tabelle 24 die Auswirkungen auf den Züchtungsertrag, die Züchtungskosten und den Züchtungsgewinn bei variiertem Testanteil. Außerdem sind zu jedem Erfolgsparameter die relativen Unterschiede zur Ausgangssituation mit aufgeführt. 
Tabelle 24: Vergleich von monetärem Zuchtfortschritt pro Jahr insgesamt $(€)$, Züchtungskosten $(€)$ und dem Züchtungsgewinn $(€)$ und den jeweiligen relativen Unterschieden (\%) zum Basisszenario (fett gedruckt) bei verschiedenen Testanteilen.

\begin{tabular}{lrrrrrr}
\hline Parameter & \multicolumn{7}{c}{ Testanteil } \\
& 0,1 & 0,2 & $\mathbf{0 , 2 5}$ & 0,3 & 0,4 & 0,5 \\
\hline monetärer ZF/Jahr ges. (€) & 9,91 & 10,21 & $\mathbf{1 0 , 3 3}$ & 10,42 & 10,59 & 10,75 \\
relativer Unterschied (\%) & $-3,97$ & $-1,06$ & $\mathbf{0}$ & $+0,96$ & $+2,61$ & $+4,16$ \\
Züchtungsertrag (€) & 43,86 & 45,88 & $\mathbf{4 6 , 7 6}$ & 47,61 & 49,25 & 50,86 \\
relativer Unterschied (\%) & $-6,20$ & $-1,88$ & $\mathbf{0}$ & $+1,81$ & $+5,32$ & $+8,76$ \\
Züchtungsgewinn $(€)$ & 14,36 & 16,49 & $\mathbf{1 7 , 4 2}$ & 18,32 & 20,07 & 21,79 \\
relativer Unterschied (\%) & $-17,56$ & $-5,33$ & $\mathbf{0}$ & $+5,16$ & $+15,21$ & $+25,08$ \\
Züchtungskosten $(€)$ & 29,49 & 29,39 & $\mathbf{2 9 , 3 3}$ & 29,28 & 29,18 & 29,07 \\
relativer Unterschied & $+0,54$ & $+0,20$ & $\mathbf{0}$ & $-0,17$ & $-0,51$ & $-0,88$ \\
\hline
\end{tabular}

Der kontinuierliche Anstieg der Erfolgsparameter Züchtungsertrag und Züchtungsgewinn bei einer größeren Testkapazität basiert zum einen darauf, dass weniger Kuhväter benötigt werden und daher eine schärfere Selektion in diesem Pfad stattfinden kann. Zudem stehen mit Erhöhung des Testumfangs mehr Töchter pro Testbulle zur Verfügung, was die Genauigkeit der Zuchtwertschätzung verbessert und somit auch den Zuchtfortschritt erhöht. Insgesamt erhöht sich der Züchtungsgewinn mit Steigerung der Testkapazität von $25 \%$ auf $50 \%$ um $4 €$ pro Kuh und Jahr, was prozentual ausgedrückt eine Zunahme von $25 \%$ entspricht. Weiterhin zeigen die Züchtungskosten minimale Veränderungen bei Variation des Testanteils. Verantwortlich dafür sind die vermehrten günstigeren Besamungen mit Testbullen. Der Anteil der Besamungen mit Altbullen geht zurück, wodurch sich die gesamten Bullenhaltungskosten für geprüfte Bullen reduzieren.

Die Tabelle 25 zeigt die monetären Auswirkungen bei Variation der Anzahl an Testbullen für das Zuchtprogramm bei einem konstanten Testanteil von $25 \%$. Dabei wird jeweils die Basisvariante mit 20 Testbullen mit 8 alternativen Varianten hinsichtlich des monetären Zuchtfortschritts pro Jahr, des Züchtungsertrags, des Züchtungsgewinns und der Züchtungskosten verglichen. 
Tabelle 25: Auswirkung auf monetären Zuchtfortschritt pro Jahr insgesamt (€), Züchtungskosten $(€)$ und dem Züchtungsgewinn (€) und relative Unterschiede (\%) zum Basisszenario (fett gedruckt) bei Variation der Anzahl der Testbullen.

\begin{tabular}{lrrrrrrrrrr}
\hline Parameter & \multicolumn{10}{c}{ Anzahl Testbullen } \\
& 10 & 15 & $\mathbf{2 0}$ & 25 & 30 & 35 & 40 & 45 & 50 \\
\hline $\begin{array}{l}\text { mon. ZF/Jahr } \\
\text { ges. } € \text { ) }\end{array}$ & 9,78 & 10,00 & $\mathbf{1 0 , 3 3}$ & 10,47 & 10,54 & 10,58 & 10,59 & 10,59 & 10,58 \\
$\begin{array}{l}\text { relativer Unter- } \\
\text { schied (\%) }\end{array}$ & $-5,23$ & $-3,10$ & $\mathbf{0}$ & $+1,45$ & $+2,13$ & $+2,51$ & $+2,61$ & $+2,61$ & $+2,51$ \\
$\begin{array}{l}\text { Züchtungsertrag } \\
(€)\end{array}$ & 43,32 & 44,50 & $\mathbf{4 6 , 7 6}$ & 48,26 & 49,78 & 50,71 & 51,30 & 51,72 & 52,00 \\
$\begin{array}{l}\text { relativer Unter- } \\
\text { schied (\%) }\end{array}$ & $-7,35$ & $-4,83$ & $\mathbf{0}$ & $+3,20$ & $+6,45$ & $+8,44$ & $+9,70$ & $+10,60$ & $+11,20$ \\
$\begin{array}{l}\text { Züchtungsgewinn } \\
(€)\end{array}$ & 16,16 & 16,25 & $\mathbf{1 7 , 4 2}$ & 17,83 & 18,26 & 18,10 & 17,60 & 16,93 & 16,11 \\
$\begin{array}{l}\text { relativer Unter- } \\
\text { schied (\%) }\end{array}$ & $-7,23$ & $-6,71$ & $\mathbf{0}$ & $+2,35$ & $+4,82$ & $+3,90$ & $+1,03$ & $-2,81$ & $-7,52$ \\
$\begin{array}{l}\text { Züchtungskosten } \\
(€)\end{array}$ & 27,15 & 28,24 & $\mathbf{2 9 , 3 3}$ & 30,43 & 31,52 & 32,61 & 33,70 & 34,79 & 35,88 \\
$\begin{array}{l}\text { relativer Unter- } \\
\text { schied (\%) }\end{array}$ & $-7,43$ & $-3,71$ & $\mathbf{0}$ & $+3,75$ & $+7,46$ & $+11,18$ & $+14,89$ & $+18,61$ & $+22,33$ \\
\hline
\end{tabular}

Tabelle 25 zeigt deutlich, dass das Testen von Testbullen mit zunehmender Anzahl an Tieren teurer wird. Die Züchtungskosten nehmen im Vergleich zum Ausgangswert von $29,33 €$ um $22 \%$ zu und belaufen sich bei 50 Testbullen auf $35,88 €$. Entscheidende Kostenfaktoren, die die Höhe der Züchtungskosten wesentlich beeinflussen, sind hierbei die Kosten für die Wartebullenhaltung, die Kosten für die Nachzuchtbewertung und die Kosten der Laktationsprämien. Aus diesem Grund und anhand eines vorliegenden optimalen Züchtungsgewinns von $18,26 €$ bei 30 Tieren, zeigt sich eine Beschränkung der Anzahl an Testbullen als vorteilhaft.

In Abbildung 24 werden beide bisher untersuchten Parameter kombiniert und die Entwicklung des Züchtungsgewinns bei variierter Anzahl an Testbullen und gleichzeitiger Veränderung des Testanteils dargestellt. 


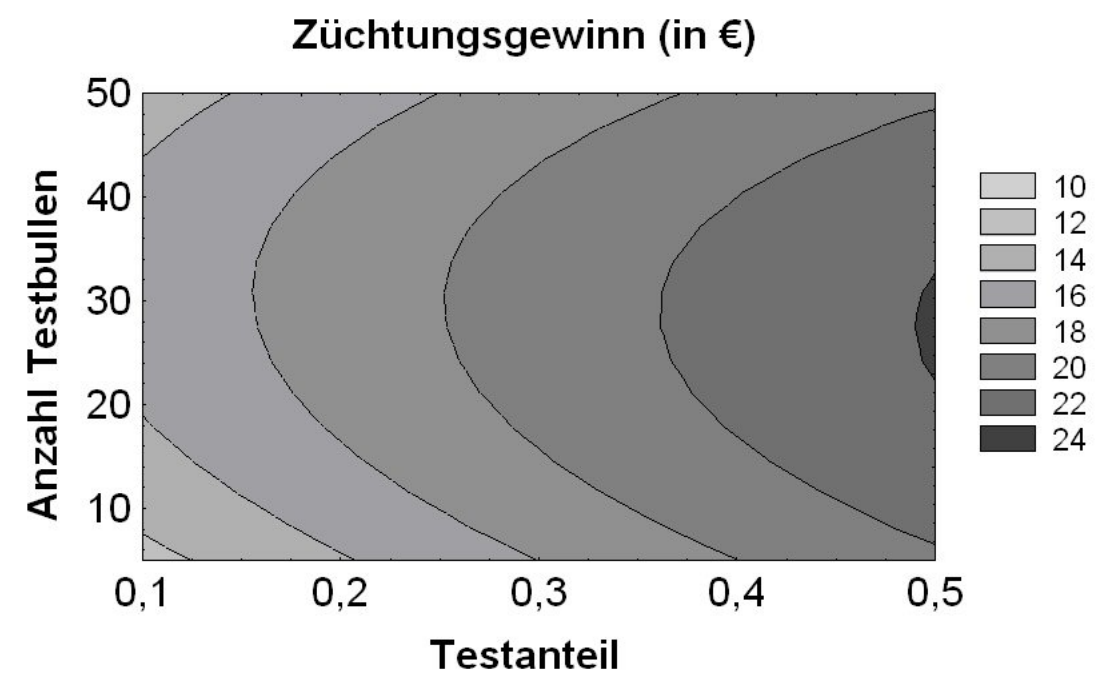

Abbildung 24: Auswirkungen auf den Züchtungsgewinn ( $€ /$ Kuh/Jahr) bei einer unterschiedlichen Anzahl an Testbullen und variiertem Testanteil im Vergleich zur Ausgangssituation mit 20 Testbullen und einem Testanteil von 0,25.

Die Skala neben der Abbildung zeigt den erwarteten Züchtungsgewinn (€) pro Kuh nach der Investitionsdauer von 20 Jahren. Wie bereits erwähnt liegt auch hier die optimale Anzahl an Testbullen bei 30 Tieren. Eine weitere Erhöhung der Testbullen auf 50 Tiere wirkt sich aufgrund der hohen Kosten negativ auf den Züchtungsgewinn aus. Da wie bereits zu Tabelle 24 beschrieben höhere Testanteile die Genauigkeit der Zuchtwertschätzung verbessern, zeigen sich auch hier bei hohen Testanteilen innerhalb einer Gruppe von Testbullen die höchsten Züchtungsgewinne. Das Optimum von 22,11 € Züchtungsgewinn pro Kuh und Investitionsperiode wird schließlich mit 30 Testbullen und einer Testkapazität von 0,5 erreicht. Niedrigere Testkapazitäten und eine hohe Anzahl an Testbullen führen entsprechend zu geringeren Zuchtfortschritten, da auch die Heritabilitäten der funktionalen Merkmale und die genetischen Korrelationen zu den Milchleistungsmerkmalen sehr gering sind. 
4.2.1.2 Veränderung der ökonomischen Gewichte in den Merkmalsgruppen Milch und Fitness

Tabelle 26 zeigt die wirtschaftlichen Gewichte der Fitnessmerkmale der einzelnen Varianten des ökologischen Zuchtprogramms. Die Gewichte sind pro genetische Standardabweichung angegeben. Das Basiszuchtprogramm mit den Ausgangsgewichten ist mit $100 \%$ angegeben. Die Verringerung um $25 \%$ und $50 \%$ sind in den Varianten $75 \%$ bzw. $50 \%$ abgebildet. Die Erhöhung um $25 \%$ und $50 \%$ ist mit $125 \%$ bzw. $150 \%$ gekennzeichnet. Da das Merkmal Totgeburtenrate züchterisch positiv zu bewerten ist, geht dies, anders als Tabelle 14 in Kapitel 3.2.3 zu entnehmen, mit einem positiven Vorzeichen in die Berechnung ein.

Tabelle 26: Verringerung und Erhöhung der wirtschaftlichen Gewichte der Fitnessmerkmale um jeweils $25 \%$ und $50 \%$ von den Ausgangsgewichten (100 \%).

\begin{tabular}{cccccccccccccc}
\hline$\%$ & Fkg & Ekg & ND & Pers & FRUp & FRUm & KVLp & KVLm & TOTp & TOTm & ZZ & DMG \\
\hline $50 \%$ & $-2,49$ & 61,71 & 15,71 & 3,01 & 6,10 & 6,10 & 0,14 & 0,14 & 0,27 & 0,27 & 2,96 & 1,21 \\
$75 \%$ & $-3,73$ & 51,42 & 23,57 & 4,51 & 9,15 & 9,15 & 0,21 & 0,21 & 0,40 & 0,40 & 4,44 & 1,81 \\
$100 \%$ & $-4,98$ & 41,14 & 31,43 & 6,02 & 12,21 & 12,21 & 0,29 & 0,29 & 0,54 & 0,54 & 5,92 & 2,42 \\
$125 \%$ & $-6,22$ & 30,85 & 39,28 & 7,52 & 15,26 & 15,26 & 0,36 & 0,36 & 0,67 & 0,67 & 7,40 & 3,02 \\
$150 \%$ & $-7,47$ & 20,57 & 47,14 & 9,03 & 18,31 & 18,31 & 0,43 & 0,43 & 0,81 & 0,81 & 8,88 & 3,63 \\
\hline
\end{tabular}

Eine Übersicht über die Auswirkungen auf den naturalen und monetären Zuchtfortschritt bei Veränderung der wirtschaftlichen Gewichte der Fitnessmerkmale zeigt Tabelle 27. Damit der naturale Zuchtfortschritt vergleichbarer und anschaulicher ist, wird dieser wie in vorigen Berechnungen standardisiert in Prozent einer genetischen Standardabweichung des jeweiligen Merkmals ausgedrückt. Der gesamte jährliche monetäre Zuchtfortschritt ergibt sich durch die Aufsummierung der einzelnen monetären Zuchtfortschritte der dargestellten Merkmale. Mit Ausnahme der Merkmale Durchschnittliches Minutengemelk und maternaler Kalbeverlauf wirkt sich eine Erhöhung der Gewichtung der Fitnessmerkmale deutlich positiv auf die einzelnen naturalen Zuchfortschritte dieser Merkmale aus. Deutliche Verbesserungen sind in den Merkmalen Nutzungsdauer, maternale Fruchtbarkeit und Zellzahl zu erkennen. Die naturalen Zuchtfortschritte für die Milchmerkmale fallen entsprechend geringer aus. Bei einer Erhöhung von bis zu $50 \%$ zeigt sich im naturalen Zuchtfortschritt für das Merkmal Fett-kg sogar ein negativer Wert von -1,82. Der Ausgangswert dieses Merkmal liegt vergleichsweise bei 13,58. 
Tabelle 27: Naturaler Zuchtfortschritt pro Jahr (in $\mathrm{s}_{A}{ }^{*} 100$ ) und monetärer Zuchtfortschritt pro Jahr gesamt der Fitnessmerkmale (monZF/Jahr gesamt, $€$ ) nach Verringerung bzw. Erhöhung der wirtschaftlichen Gewichte der Fitnessmerkmale um jeweils $25 \%$ und $50 \%$ im Vergleich zum Basisszenario (fett gedruckt).

\begin{tabular}{lrrrrrr}
\hline Merkmal & Einheit & $50 \%$ & $75 \%$ & $\mathbf{1 0 0} \%$ & $125 \%$ & $150 \%$ \\
\hline Fkg & $\mathrm{kg}$ & 20,78 & 18,36 & $\mathbf{1 3 , 5 8}$ & 6,26 & $-1,82$ \\
Ekg & $\mathrm{kg}$ & 24,72 & 22,41 & $\mathbf{1 7 , 4 8}$ & 9,63 & 0,61 \\
& & & & & & \\
ND & Tage & 1,69 & 5,35 & $\mathbf{9 , 7 4}$ & 13,51 & 15,47 \\
Pers & $\mathrm{S}_{\mathrm{A}}$ & 2,22 & 4,12 & $\mathbf{6 , 4 6}$ & $\mathbf{8 , 6 2}$ & 9,59 \\
FRUp & $\%$ & $-2,19$ & $-1,71$ & $\mathbf{- 0 , 8 5}$ & 0,41 & 1,60 \\
FRUm & $\%$ & $-3,06$ & $-0,68$ & $\mathbf{2 , 6 2}$ & 6,26 & 9,02 \\
KVLp & Klasse & $-2,79$ & $-2,68$ & $\mathbf{- 2 , 3 2}$ & $-1,60$ & $-0,67$ \\
KVLm & Klasse & 3,81 & 4,42 & $\mathbf{4 , 8 8}$ & 4,83 & 4,13 \\
TOTp & $\%$ & $-0,08$ & $-0,05$ & $-\mathbf{0 , 0 4}$ & $-0,01$ & 0,02 \\
TOTm & $\%$ & 1,01 & 1,76 & $\mathbf{2 , 6 3}$ & 3,34 & 3,60 \\
ZZ & Klasse & $-4,93$ & $-2,91$ & $\mathbf{0 , 1 4}$ & 3,89 & 7,09 \\
DMG & kg/min & 6,37 & 5,95 & $\mathbf{4 , 8 5}$ & 2,90 & 0,57 \\
\hline mon ZF/Jahr gesamt & & 14,68 & 12,05 & $\mathbf{1 0 , 3 3}$ & 9,96 & 11,06 \\
\hline
\end{tabular}

Nach der Reduzierung der ökonomischen Gewichte der Fitnessmerkmale zeigen die Milchmerkmale Fett- und Eiweißmenge deutlich höhere Zuchtfortschritte. Die naturalen Zuchtfortschritte der Fitnessmerkmale nehmen, vergleichsweise zum Basiswert, bei einer Reduzierung ab. Sehr deutlich wird dieser Rückgang im Merkmal Zellzahl. Der anfangs positive Zuchtfortschritt entwickelt sich zu einem negativen Zuchtfortschritt. Bei einer Reduzierung der ökonomischen Gewichte der Fitnessmerkmale wird ein höherer monetärer Zuchtfortschritt erzielt, als bei einer Erhöhung der ökonomischen Bedeutung dieses Merkmalkomplexes. Aufgrund der höheren Gewichtung der Fitnessmerkmale wäre ein höherer gesamter monetärer Zuchtfortschritt pro Jahr zu erwarten, als der gezeigte geringfügige Anstieg. Tatsächlich liefert jedoch eine Reduzierung der ökonomischen Gewichte der Fitnessmerkmale um $50 \%$ und die dadurch gleichzeitig zunehmende Bedeutung der Milchmerkmale einen mit Abstand größten Beitrag zum gesamten monetären Zuchtfortschritt pro Jahr. 
Die monetären Zuchtfortschritte der Merkmalskomplexe Milch und Fitness werden in Tabelle 28 durch die Darstellung der relativen Gewichtung der Merkmalsgruppen in den jeweiligen Varianten ergänzt.

Tabelle 28: Monetärer Zuchtfortschritt pro Jahr der Merkmalsgruppen Milch und Fitness bei Verringerung bzw. Erhöhung der wirtschaftlichen Gewichte der Fitnessmerkmale um jeweils $25 \%$ und $50 \%$; Relativer Anteil einer Merkmalgruppe am gesamten monetären Zuchtfortschritt pro Jahr (€).

\begin{tabular}{lccc}
\hline Variante & \multicolumn{2}{c}{ Milch } & Fitness \\
\hline $50 \%$ & $14,73(100,34)$ & $-0,05 \quad(-0,34)$ \\
$75 \%$ & $10,83(89,87)$ & $1,22 \quad(10,13)$ \\
$100 \%$ & 6,51 & $(63,08)$ & $3,81 \quad(36,92)$ \\
$125 \%$ & 2,58 & $(25,90)$ & $7,38 \quad(74,10)$ \\
$150 \%$ & 0,26 & $(2,35)$ & $10,80(97,65)$ \\
\hline
\end{tabular}

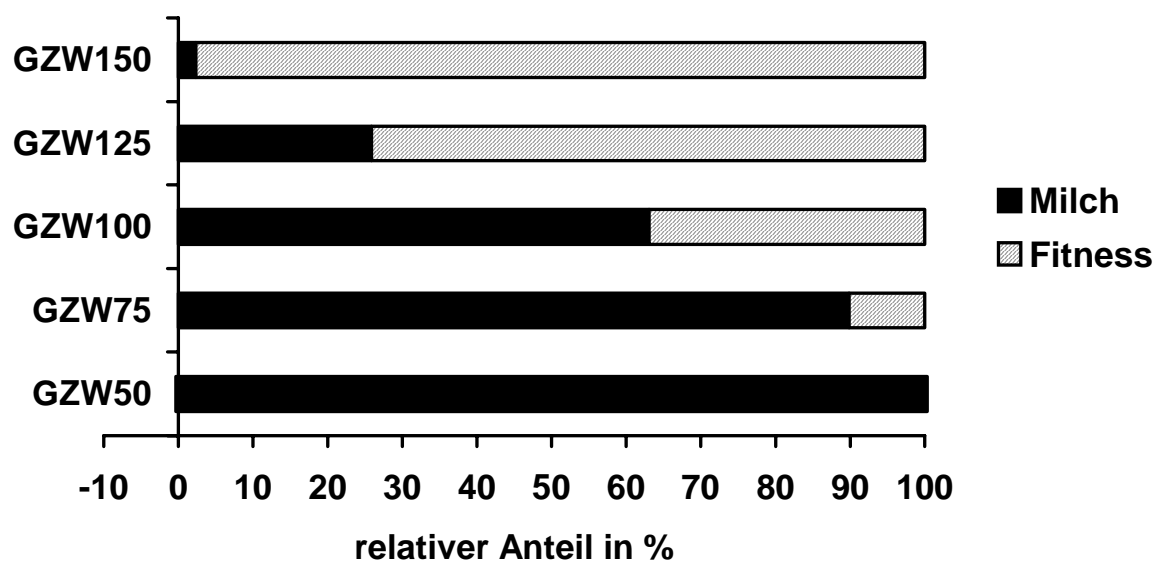

Abbildung 25: Relativer Anteil der Merkmalsgruppen am gesamten monetären Zuchtfortschritt pro Jahr bei Verringerung bzw. Erhöhung der wirtschaftlichen Gewichte der Fitnessmerkmale.

Die Tabelle 28 und auch Abbildung 25 zeigen deutlich, dass eine Verringerung bzw. Erhöhung der wirtschaftlichen Gewichte der Fitnessmerkmale zu einer Verschiebung im jeweiligen Merkmalskomplex führt. Bei einer Erhöhung um $25 \%$ bzw. 50 \% entwickelt sich die relative ökonomische Bedeutung zugunsten der Fitnessmerkmale. Diese nehmen dabei um $38 \%$ bzw. $61 \%$ zu. Analog nimmt die Merkmalsgruppe Milch um $4 \%$ und $6 \%$ ab. Eine Verringerung der Fitnessmerkmale führt zu einem Rückgang des monetären Zuchtfortschritts um etwa $26 \%$ und etwa $36 \%$. Aufgrund der dadurch entstehenden ge- 
ringeren wirtschaftlichen Bedeutung des Fitnesskomplexes nimmt die relative Gewichtung der Milchmerkmale um etwa $4 \%$ und $8 \%$ zu. Das stärkere Gewicht für Fett- und Eiweißmenge ergibt für diesen Merkmalskomplex den größten relativen Anteil am gesamt erzielten monetären Zuchtfortschritt.

\subsubsection{Veränderung der Populationsgröße im ökologischen Zuchtprogramm}

Die Ausgangspopulation des ökologischen Zuchtprogramms beträgt 109.600 Tiere. Dieser Referenzsituation werden sechs alternative Zuchtprogramme mit unterschiedlichen Populationsgrößen gegenübergestellt. Dabei wurde nur die Population von 50.000 bis zu 500.000 Tiere variiert, alle übrigen Eingabeparameter werden konstant gehalten. Tabelle 29 zeigt zu jedem Zuchtprogramm den Einfluss der Populationsgröße auf den naturalen Zuchtfortschritt der einzelnen Merkmale und den gesamten monetären Zuchtfortschritt pro Jahr.

Aus den Ergebnissen geht hervor, dass die Zunahme der Populationsgröße unterschiedliche Entwicklungen in den einzelnen Merkmalen hervorruft. So zeigen besonders die Milchmerkmale eine positive Entwicklung des naturalen Zuchtfortschrittes mit steigender Populationsgröße. Aber auch die Merkmale Nutzungsdauer, Persistenz, maternale Fruchtbarkeit, maternaler Kalbeverlauf, maternale Totgeburtenrate und das durchschnittliche Minutengemelk verbessern sich bei steigender Tierzahl. Anders verhalten sich die paternalen Merkmale Fruchtbarkeit, Kalbeverlauf und Totgeburtenrate. Die naturalen Zuchtfortschritte dieser Merkmale verschlechtern sich minimal in einer Population die größer ist als die Basispopulation mit 109.600 Tieren. Eine gegenläufige Entwicklung der naturalen Zuchtfortschritte dieser paternalen Merkmale zeigt eine Verkleinerung der Populationsgröße. Die Merkmale haben zwar noch ein negatives Vorzeichen, dennoch sind minimale Verbesserungen des naturalen Zuchtfortschrittes zu erkennen. Eindeutig auffälliger verhält sich das Merkmal Zellzahl. Die Zunahme des naturalen Zuchtfortschritts bei Erhöhung der Populationsgröße und die Reduzierung bei Verkleinerung der Population zeigen die deutlichsten Reaktionen. So liegt der naturale Zuchtfortschritt bei einer Population mit 50.000 Tieren bei $-0,04$ und bei 500.000 Tieren bei $+0,29$. Vergleichsweise dazu wird mit der Basispopulation ein Wert von 0,14 erreicht. 
Tabelle 29: Einfluss verschiedener Größen der ökologischen Milchviehpopulation auf den naturalen Zuchtfortschritt (in $\mathrm{S}_{\mathrm{A}}$ * 100) und den gesamten monetären Zuchtforschritt pro Jahr $(€)$ im Vergleich zur Ausgangspopulation (fett gedruckt).

\begin{tabular}{lrrrrrrr}
\hline & \multicolumn{7}{c}{ Populationsgröße } \\
Merkmal & 50.000 & 75.000 & $\mathbf{1 0 9 . 6 0 0}$ & 150.000 & 200.000 & 250.000 & 500.000 \\
\hline Fkg & 13,19 & 13,40 & $\mathbf{1 3 , 5 8}$ & 13,71 & 13,82 & 13,91 & 14,16 \\
Ekg & 16,94 & 17,23 & $\mathbf{1 7 , 4 8}$ & 17,65 & 17,80 & 17,91 & 18,23 \\
& & & & & & & \\
ND & & & & & & & \\
Pers & 8,66 & 9,26 & $\mathbf{9 , 7 4}$ & 10,09 & 10,37 & 10,56 & 11,04 \\
FRUp & 6,19 & 6,34 & $\mathbf{6 , 4 6}$ & 6,54 & 6,60 & 6,65 & 6,77 \\
FRUm & $-0,79$ & $-0,82$ & $-\mathbf{0 , 8 5}$ & $-0,87$ & $-0,89$ & $-0,90$ & $-0,94$ \\
KVLp & 2,01 & 2,34 & $\mathbf{2 , 6 2}$ & 2,83 & 3,00 & 3,12 & 3,42 \\
KVLm & $-2,25$ & $-2,28$ & $\mathbf{- 2 , 3 2}$ & $-2,34$ & $-2,35$ & $-2,37$ & $-2,40$ \\
TOTp & 4,67 & 4,79 & $\mathbf{4 , 8 8}$ & 4,95 & 5,00 & 5,03 & 5,13 \\
TOTm & $-0,05$ & $-0,04$ & $-\mathbf{0 , 0 4}$ & $-0,04$ & $-0,04$ & $-0,03$ & $-0,05$ \\
ZZ & 2,49 & 2,57 & $\mathbf{2 , 6 3}$ & 2,67 & 2,71 & 2,73 & 2,79 \\
DMG & $-0,04$ & 0,06 & $\mathbf{0 , 1 4}$ & 0,19 & 0,22 & 0,24 & 0,29 \\
\hline mon ZF/Jahr gesamt & $\mathbf{2 , 6 3}$ & 10,03 & $\mathbf{1 0 , 3 3}$ & 10,53 & 10,70 & 10,82 & 11,13 \\
\hline & 4,69 & 4,78 & $\mathbf{4 , 8 5}$ & 4,91 & 4,95 & 4,98 & 5,07 \\
\hline
\end{tabular}

Im gesamten monetären Zuchtfortschritt pro Jahr ist ein kontinuierlicher Anstieg zu erkennen. Mit einer Population von 50.000 Tieren wird pro Jahr mit 9,68€ der geringste monetärer Zuchtfortschritt erzielt. Dementsprechend wird der höchste monetäre Zuchtfortschritt von $11,13 €$ mit der hier maximal gewählten Populationsgröße von 500.000 Tieren erreicht. Das Basiszuchtprogramm erreicht jährlich 10,33€ monetären Zuchtfortschritt.

In Abbildung 26 sind ergänzend zu Tabelle 29 die prozentualen Veränderungen der jeweiligen naturalen Zuchtfortschritte in den Merkmalen dargestellt, wenn die Populationsgröße von 109.600 Tieren auf 200.000 Tiere erhöht wird. Die Verbesserung v. a. in den funktionalen Merkmalen lässt sich auf die höheren Sicherheiten bei der Zuchtwertschätzung der Bullen und der dadurch möglichen genaueren Selektion in diesem Pfad zurückführen. 


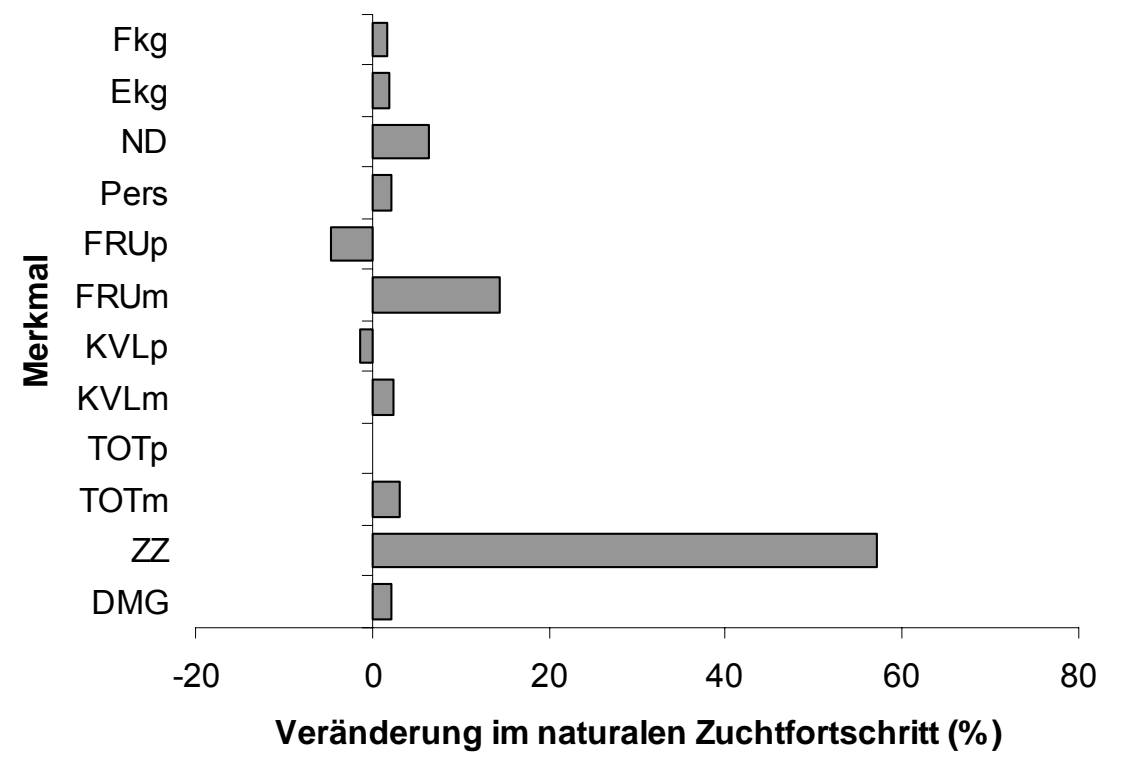

Abbildung 26: Prozentuale Veränderung der naturalen Zuchtfortschritte der einzelnen Merkmale bei Erhöhung der Populationsgröße von 109.600 auf 200.000 Tiere.

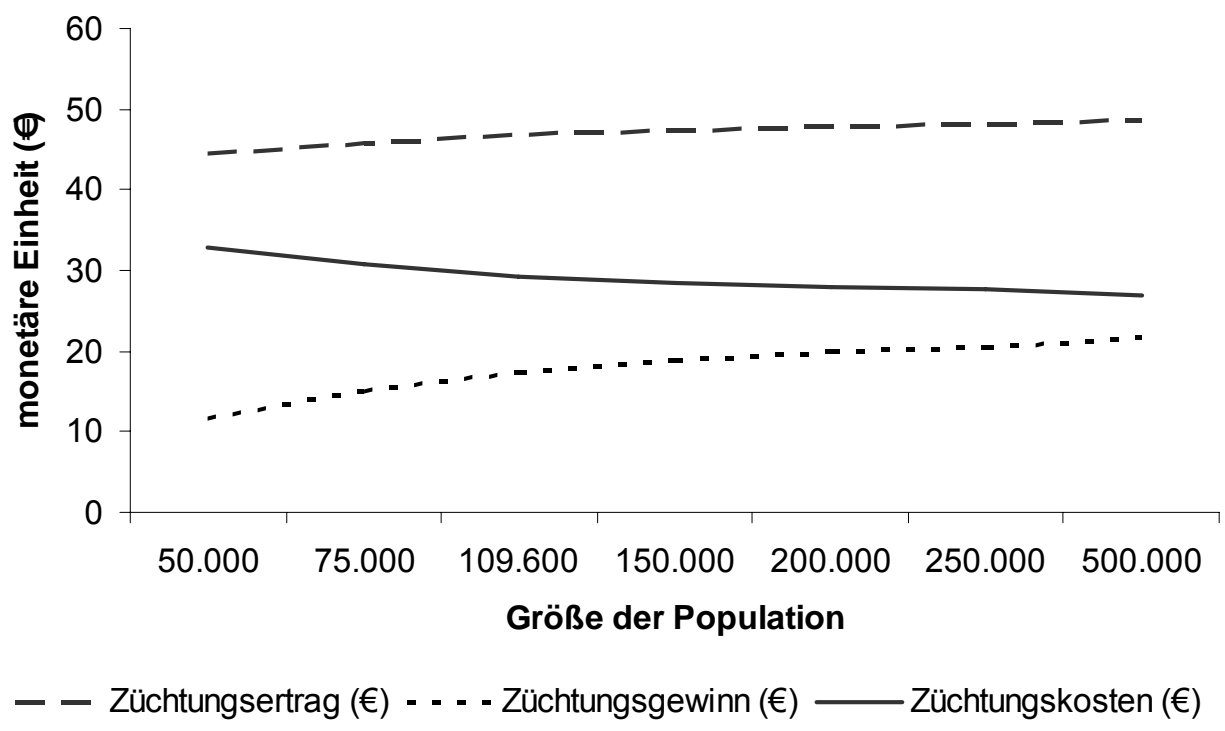

Abbildung 27: Vergleich des Züchtungsertrags $(€)$, des Züchtungsgewinns $(€)$ und der Züchtungskosten $(€)$ bei unterschiedlicher Populationsgröße.

Die überwiegend positive Entwicklung der Merkmale (vgl. Abbildung 26), aber auch die in Abbildung 27 gezeigten positiven Entwicklungen im Züchtungsertrag, dem Züchtungsgewinn und den Züchtungskosten, sind auf eine verbesserte Selektion der Bullenmütter bei Erhöhung der Populationsgröße zurückzuführen. Da diesen Zuchtprogrammen eine konstante Anzahl an Testbullen mit konstanter Testkapazität unterstellt wurde, können die 
variablen Kosten in einer größeren Population auf mehr Tiere umgelegt werden. Diese auch in Abbildung 27 gezeigte Kostendegression führt bei ansteigendem Züchtungsertrag zu einer Zunahme im Züchtungsgewinn.

\subsubsection{Töchterleistung pro Anzahl Besamungen}

Für das ökologische Basiszuchtprogramm wurde ein Ausgangsverhältnis von 1:10 gewählt. Zum Vergleich wurden bessere aber auch schlechtere Verhältnisse von 1:4 bis 1:20 modelliert.

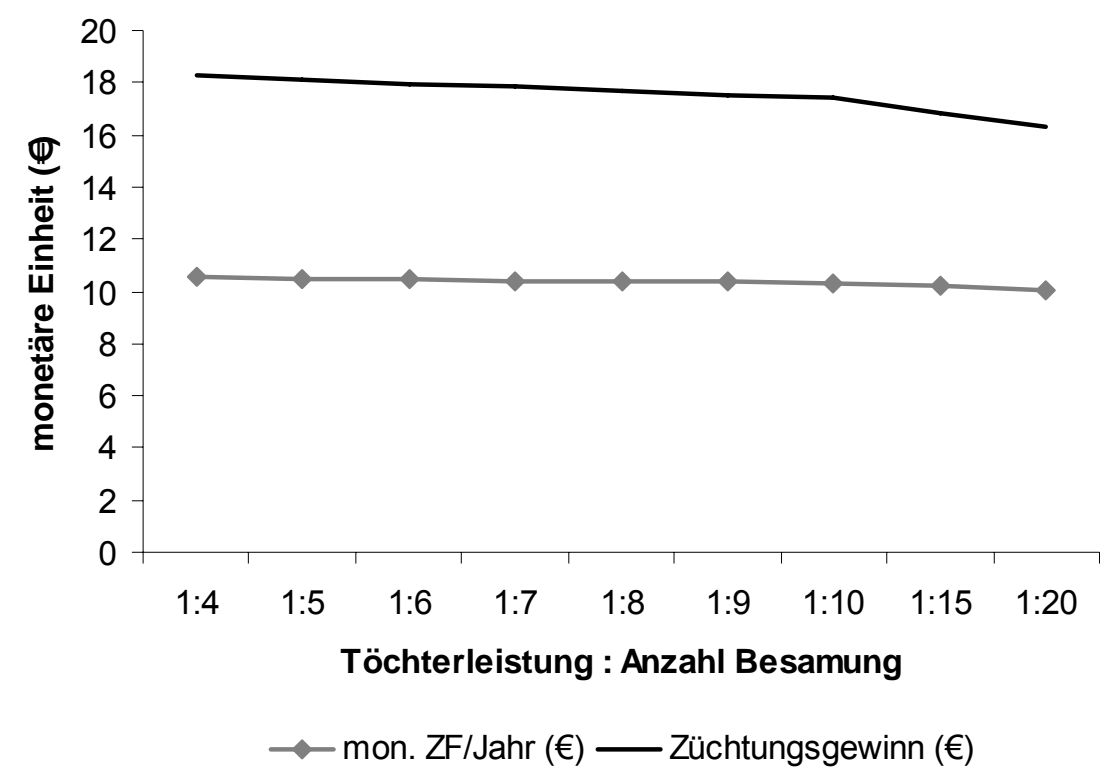

Abbildung 28: Variation der Anzahl Besamungen pro Töchterleistung und die sich dadurch ergebenden Unterschiede im monetären Zuchtfortschritt pro Jahr insgesamt $(€)$ und dem Züchtungsgewinn $(€)$ im Vergleich zum Ausgangsverhältnis.

Abbildung 28 zeigt detailliert, wie der monetäre Zuchtfortschritt pro Jahr gesamt (€) und der Züchtungsgewinn $(€)$ von unterschiedlichen Verhältnissen der Töchterleistung pro Anzahl Besamungen beeinflusst werden. Die Ergebnisse zeigen für den monetären Zuchtfortschritt im Jahr weniger große Unterschiede im Betrag. Der höchste monetäre Zuchtfortschritt mit 10,53€ und auch der höchste Züchtungsgewinn in Höhe von 18,27 € werden mit dem besten eingesetzten Verhältnis von 1:4 erreicht. Durch diese hohe Wiederfindungsrate können mehr Töchterinformationen gewonnen werden als vergleichsweise zum Basisverhältnis. Das bedeutet, dass sich mit engem Verhältnis die Genauigkeit der Zuchtwertschätzung verbessert und bei einem weiten Verhältnis entsprechend verschlechtert. Zudem wird der Züchtungsgewinn maßgeblich durch die Züchtungskosten und hier speziell durch die Kosten zur Produktion von Sperma beeinflusst. Da die Spermaproduktion bei einem guten Verhältnis der gewonnen Töchterleistung pro Anzahl Be- 
samung eingeschränkt werden kann, reduzieren sich die Züchtungskosten und der Züchtungsgewinn steigt an. Der geringste Züchtungsgewinn von 16,33€ und auch der kleinste monetäre Zuchtfortschritt von $10,07 €$ werden folglich bei dem schlechtesten Verhältnis von 1:20 erzielt.

4.2.1.5 Variation des Verhältnisses der Anteile an künstlicher Besamung in der Zucht- und Produktionsstufe

In diesem Kapitel werden die Ergebnisse der Erfolgsparameter des ökologischen Zuchtprogramms analysiert, nachdem der Einsatz an künstlicher Besamung mit unterschiedlichen Anteilen und in verschiedenen Verhältnissen auf die Zucht- und Produktionsstufe aufgeteilt wurde. Ausgehend von einem für das Basiszuchtprogramm gewählten Verhältnis von 88 \% künstlicher Besamung in der Zuchtstufe und $80 \%$ in der Produktionsstufe, wurden jeweils die Anteile in der Zuchtstufe variiert und der Anteil in der Produktionsstufe auf $80 \%$ fixiert. Abbildung 29 zeigt die Ergebnisse für den monetären Zuchtfortschritt pro Jahr $(€)$, den Züchtungsertrag $(€)$, Züchtungskosten $(€)$ und den Züchtungsgewinn (€).

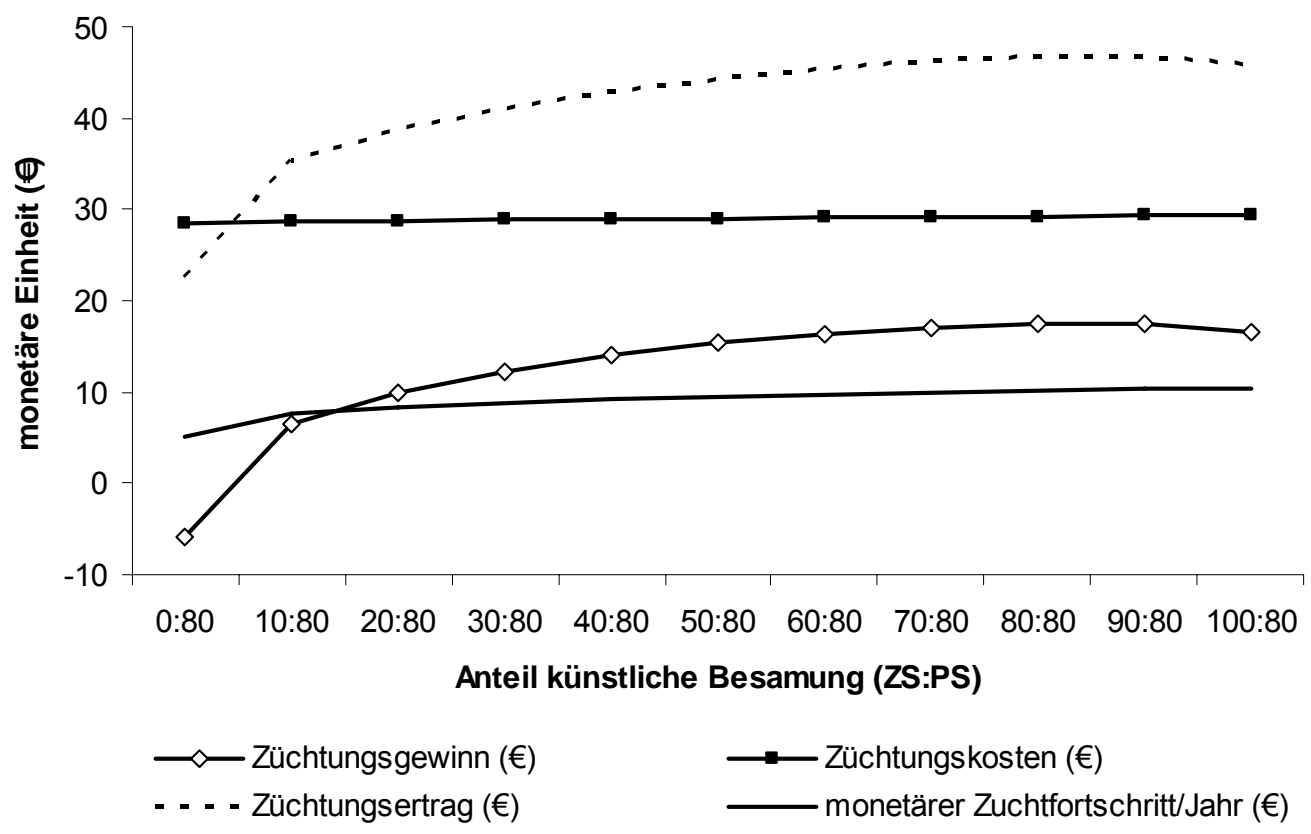

Abbildung 29: Auswirkungen auf den Züchtungsgewinn $(€)$, den Züchtungsertrag $(€)$, den gesamten monetären Zuchtfortschritt pro Jahr $(€)$ und den Züchtungskosten $(€)$ bei unterschiedlichen Anteilen der künstlichen Besamung (\%) sowohl in der Zuchtstufe als auch in der Produktionsstufe (ZS:PS).

Hier ist deutlich zu erkennen, dass bei Verringerung des Anteils an künstlicher Besamung in der Zuchtstufe alle Parameter kontinuierlich abnehmen. Der höchste Züchtungsgewinn 
in Höhe von 17,43€ wird in dieser Untersuchung mit einem Verhältnis von 80:80, d.h. mit $80 \%$ künstlicher Besamung in der Zuchtstufe und $80 \%$ in der Produktionsstufe erreicht. Wird in der Zuchtstufe ganz auf den Einsatz künstlicher Besamung verzichtet und nur in der Produktionsstufe eingesetzt, zeigt sich ein starker Verlust im Züchtungsgewinn in Höhe von $-5,99 €$, da die Kosten höher liegen als der Ertrag. Die Verringerung des Anteils künstlicher Besamung in der Zuchtstufe führt zur Reduzierung der aktiven Zuchtpopulation und zur Verringerung der Selektionsintensität bei Natursprungbullen. Da somit weniger Töchter für die Zuchtwertschätzung zur Verfügung stehen, nehmen alle Erfolgsparameter kontinuierlich ab. Mit zunehmendem Anteil an künstlicher Besamung in der Zuchtstufe steigen aber auch wieder die Züchtungskosten an, da mehr Kosten für Spermalagerung, Produktion und Wartehaltung der Bullen aufgewendet werden müssen. Die Züchtungskosten werden somit hauptsächlich durch die Zuchtstufe beeinflusst.

\subsubsection{Unterschiede der Verteilung der Kühe auf die Zucht- und Produktionsstufe}

In diesem Kapitel wird dargestellt, wie sich eine prozentuale Umverteilung der Kühe auf Zucht- und Produktionsstufe auf den gesamten monetären Zuchtfortschritt pro Jahr, den Züchtungsertrag, die Züchtungskosten und den Züchtungsgewinn auswirkt. Die für die Basisvariante des ökologischen Zuchtprogramms gewählte Verteilung zeigt 80 \% der Kühe in der Zuchtstufe und entsprechend $20 \%$ in der Produktionsstufe. Um die alternativen Varianten vergleichen zu können, ist die Basis in Tabelle 30 fett gedruckt.

Tabelle 30: Unterschiedliche Verteilung der Anzahl Kühe in der Zucht- und Produktionsstufe (in \%) und die Auswirkung auf den monetären Zuchtfortschritt pro Jahr gesamt $(€)$, den Züchtungsertrag $(€)$, die Züchtungskosten $(€)$ und den Züchtungsgewinn (€) im Vergleich zum Basisszenario (fett gedruckt).

\begin{tabular}{lrrrrrrrrr}
\hline & & \multicolumn{7}{c}{ Verteilung der Kühe (\%) } \\
$\begin{array}{lrrrrrr}\text { Parameter } \\
(€)\end{array}$ & ZS & 10 & 30 & 50 & 60 & 70 & $\mathbf{8 0}$ & 90 & 100 \\
& PS & 90 & 70 & 50 & 40 & 30 & $\mathbf{2 0}$ & 10 & 0 \\
\hline mon ZF/Jahr ges. & 8,34 & 9,49 & 9,96 & 10,11 & 10,22 & $\mathbf{1 0 , 3 3}$ & 10,40 & 10,47 \\
Züchtungsertrag & 21,46 & 29,81 & 37,10 & 40,44 & 43,64 & $\mathbf{4 6 , 7 6}$ & 49,76 & 52,59 \\
Züchtungskosten & 6,27 & 12,86 & 19,45 & 22,75 & 26,04 & $\mathbf{2 9 , 3 3}$ & 32,63 & 35,92 \\
Züchtungsgewinn & 15,18 & 16,95 & 17,64 & 17,69 & 17,60 & $\mathbf{1 7 , 4 2}$ & 17,13 & 16,66 \\
\hline
\end{tabular}


Eine Verteilung der Kühe zu Gunsten der Zuchtstufe führt zu einem linearen Anstieg des monetären Zuchtfortschrittes, des Züchtungsertrages und der Züchtungskosten, da durch eine stärkere Selektionsintensität in der Zuchtstufe mehr Selektionserfolg erreicht wird als in der Produktionsstufe. Der Züchtungsgewinn, der wichtigste Parameter, verhält sich eher gegenläufig. Die beste Aufteilung bzw. der höchste Züchtungsgewinn von 17,69€ wird dann erreicht, wenn $60 \%$ der Kühe auf die Zuchtstufe und $40 \%$ auf die Produktionsstufe verteilt werden. Bei mehr als $40 \%$ Kühe in der Produktionsstufe und mehr als $60 \%$ in der Zuchtstufe nimmt der Züchtungsgewinn ab. Trotzdem zeigt sich die Tendenz zu mehr Gewinn, wenn der Anteil an Kühen in der Zuchtstufe höher ist als in der Produktionsstufe.

\subsubsection{Alter der Kühe bei Geburt der ersten Nachkommen}

Ein weiterer wichtiger Faktor, das ökologische Zuchtprogramm zu bewerten, ist die Entwicklung der Erfolgsparameter bei unterschiedlichem Erstkalbealter der Kühe. In Tabelle 31 wurde das durchschnittliche Alter der Kühe bei der Geburt der ersten Nachkommen zwischen zwei und vier Jahren variiert. Da ein höheres Alter unrealistisch ist, wurden keine weiteren Untersuchungen mit höherem Erstkalbealter durchgeführt. Für das Basiszuchtprogramm wurde ein durchschnittliches Alter der Kühe von 2,6 Jahren gewählt. Die entsprechenden Basisergebnisse sind fett gedruckt. Bis auf die Züchtungskosten, die für jedes Alter konstant bei 29,33€ liegen, gehen alle weiteren Parameter mit zunehmenden Alter kontinuierlich zurück. Der höchste gesamte monetäre Zuchtfortschritt, der pro Jahr erzielt werden kann, liegt bei 10,70€. Dieser Betrag wird beim geringsten Erstkalbealter von zwei Jahren erreicht. Bei einem höheren Alter geht der monetäre Zuchtfortschritt weiter zurück. Gleiches zeichnet sich bei Betrachtung des Züchtungsertrages ab. Dieser ist aufgrund einer besseren Selektion und einem kürzeren Generationsintervall bei einem Alter von nur zwei Jahren mit 51,54€ am höchsten.

Je niedriger das durchschnittliche Alter der Kühe bei Geburt der ersten Nachkommen ist, desto höher ist auch der Züchtungsgewinn. Die Basisvariante des Zuchtprogramms gewinnt bei einem durchschnittlichen Erstkalbealter von 2,6 Jahren 17,42 €. Noch höher ist der Züchtungsgewinn bei nur zwei Jahren, da hier die meisten Einsparungen in den Produktionskosten bei der Rinderaufzucht und in den Remontierungskosten der gesamten Herde gemacht werden können. 
Tabelle 31: Durchschnittliches Alter der Kühe bei der Geburt der ersten Nachkommen (Jahre). Vergleich des monetären Zuchtfortschrittes pro Jahr insgesamt $(€)$, des Züchtungsertrags $(€)$, der Züchtungskosten $(€)$ und des Züchtungsgewinns $(€)$ im Vergleich zum Basisszenario (fett gedruckt).

\begin{tabular}{|c|c|c|c|c|c|c|c|c|c|c|c|}
\hline \multirow[b]{2}{*}{ Parameter (€) } & \multicolumn{11}{|c|}{ Alter der Kühe im ø } \\
\hline & 2,0 & 2,2 & 2,4 & 2,6 & 2,8 & 3,0 & 3,2 & 3,4 & 3,6 & 3,8 & 4,0 \\
\hline $\begin{array}{l}\text { mon ZF/Jahr } \\
\text { ges. }\end{array}$ & 10,70 & 10,57 & 10,44 & 10,32 & 10,2 & 10,08 & 9,96 & 9,85 & 9,74 & 9,63 & 9,52 \\
\hline $\begin{array}{l}\text { Züchtungs- } \\
\text { ertrag }\end{array}$ & 51,54 & 49,91 & 48,31 & 46,76 & 45,26 & 43,82 & 42,46 & 41,14 & 39,84 & 38,60 & 37,41 \\
\hline $\begin{array}{l}\text { Züchtungs- } \\
\text { kosten }\end{array}$ & 29,33 & 29,33 & 29,33 & 29,33 & 29,33 & 29,33 & 29,33 & 29,33 & 29,33 & 29,33 & 29,33 \\
\hline $\begin{array}{l}\text { Züchtungs- } \\
\text { gewinn }\end{array}$ & 22,21 & 20,57 & 18,97 & 17,42 & 15,92 & 14,48 & 13,13 & 11,8 & 10,5 & 9,27 & 8,07 \\
\hline
\end{tabular}

In Abbildung 30 ist zusätzlich die durchschnittliche Verringerung des Züchtungsgewinns pro zusätzlichen Monat Erstkalbealter dargestellt. Dabei zeigt die y-Achse den eben beschriebenen Züchtungsgewinn und auf der x-Achse sind die untersuchten Erstkalbealter in Monaten abgetragen. In dieser Abbildung ist der annähernd linearen Rückgang des Züchtungsgewinns in Höhe von $-0,589 €$ mit Zunahme des Erstkalbealters je zusätzlicher Monat deutlich zu erkennen.

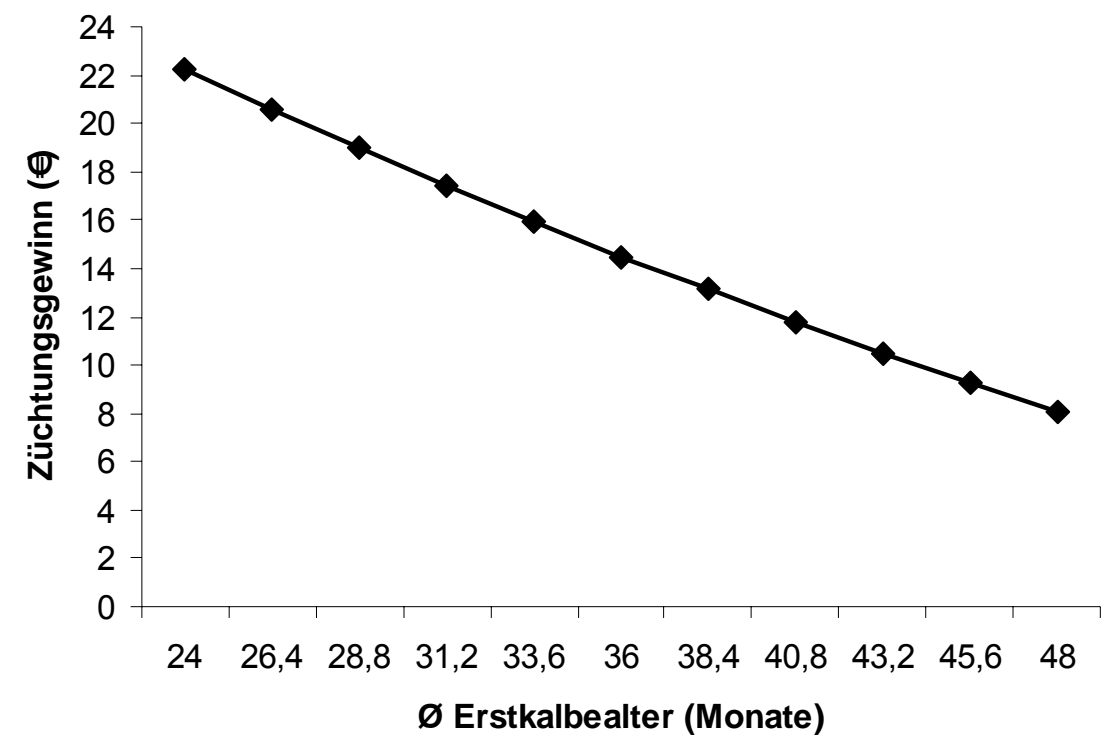

Abbildung 30: Züchtungsgewinns (€) in Abhängigkeit vom durchschnittlichen Erstkalbealter. 


\subsubsection{Ergebnisse der Untersuchungen zu Genotyp-Umwelt-Interaktionen im öko- logischen Zuchtprogramm}

In den nachfolgenden Kapiteln werden die Auswirkungen der Genotyp-UmweltInteraktionen für das Merkmal Eiweißmenge, Zellzahl und Nutzungsdauer überprüft. Dafür wurden drei verschiedene Zuchtprogramme modelliert. Anschließend wurde ein geschlossenes Ökozuchtprogramm einem offenen Ökozuchtprogramm mit $20 \%$ bzw. $40 \%$ Genfluss durch den Einsatz von im konventionellen Zuchtprogramm selektierten Bullen gegenübergestellt. Das geschlossene Zuchtprogramm ist in den folgenden Abbildungen mit $100 \%$ gekennzeichnet, das Programm mit $20 \%$ konventionellem Genfluss ist durch $80 \%$ gekennzeichnet und das Programm mit $40 \%$ Genfluss entsprechend mit $60 \%$. In jedem Programm wurden unterschiedliche genetische Korrelationen zwischen dem jeweiligen ökologischen und konventionellen Merkmal eingesetzt. Anschließend wurden auch unterschiedliche Heritabilitäten für das zu untersuchende Merkmal angenommen und auf gleiche Weise eine Schätzung der Auswirkungen der Genotyp-Umwelt-Interaktion vorgenommen. Die Ergebnisse wurden nach dem naturalen Zuchtfortschritt und dem Züchtungsertrag bewertet und beziehen sich wie alle weiteren Parameter ausschließlich auf die Ökopopulation.

\subsubsection{Genotyp-Umwelt-Interaktionen beim Merkmal Eiweißmenge}

Abbildung 31 zeigt die Auswertung der Genotyp-Umwelt-Interaktion für das Merkmal Eiweiß-kg bei einer Heritabilität von 0,34. Die genetische Korrelation für dieses Merkmal wurde in allen drei Zuchtprogrammen von 1,0 bis 0,8 variiert. Die dargestellten Balken setzen sich aus den Züchtungskosten und dem Züchtungsgewinn zusammen. Sie zeigen insgesamt den durchschnittlichen Züchtungsertrag pro Kuh über den gesamten Investitionszeitraum, der im jeweiligen modellierten Zuchtprogramm erreicht werden kann. Wie erwartet verändert sich der Züchtungsertrag bei einem geschlossenen Zuchtprogramm und unterschiedlichen genetischen Korrelationen nicht und erreicht mit $25 €$ den höchsten Wert. Wird jedoch eine realistische Korrelation von 0,9 unterstellt, so geht der Züchtungsertrag im Zuchtprogramm mit $20 \%$ und $40 \%$ Genfluss zurück, da bei einem offenen ÖkoZuchtprogramm die Ökopopulation verkleinert und durch eine geringere Anzahl an Nachkommen weniger Züchtungsertrag realisiert wird. 


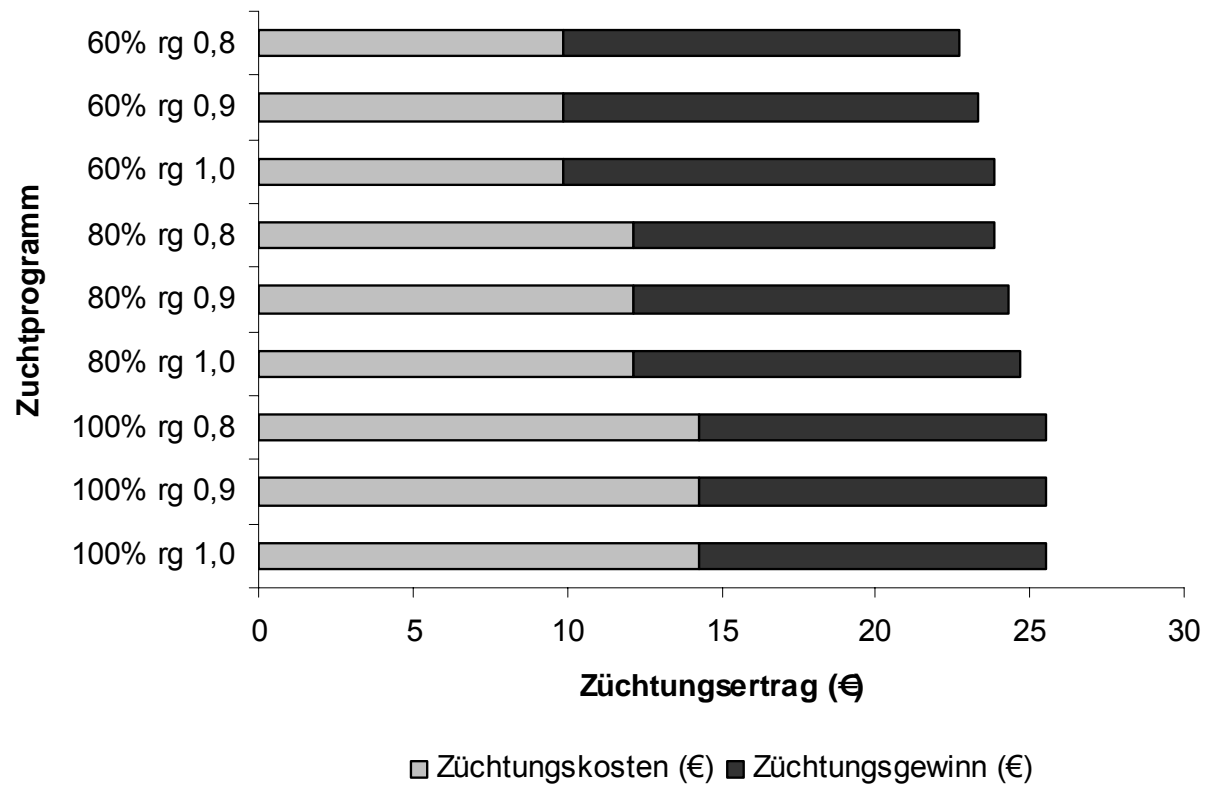

Abbildung 31: Züchtungsertrag (€) für das Merkmal Zellzahl in unterschiedlichen Zuchtprogrammen mit verschiedenen genetischen Korrelationen ( $\mathrm{rg}$ ) und $\mathrm{h}^{2}=0,34$.

Bei einer genetischen Korrelation von 0,8 zeigt sich der gleiche Effekt auf den Züchtungsertrag in allen Zuchtprogrammen. Innerhalb eines Zuchtprogramms nimmt der Züchtungsertrag bei kleiner werdenden genetischen Korrelationen ab, da sich der Anteil an konventionellen Bullen erhöht. Weiterhin ist zu erkennen, dass die offenen Zuchtprogramme deutlich geringere Züchtungskosten haben. Je mehr konventioneller Einfluss, umso geringer die Züchtungskosten, da bei Verringerung des Ökoanteils weniger Bullen auf ökologischer Seite getestet werden und die Kosten des konventionellen Zuchtprogramms nicht mitberücksichtigt werden. Die Zunahme des konventionellen Anteils zeigt auch im Züchtungsgewinn keinen Profit für die Ökopopulation, da beide Populationen unterschiedliche Zuchtziele verfolgen. Pro Kuh und Jahr liegt der maximale Züchtungsgewinn im offenen Zuchtprogramm bei $14,02 €$ verglichen mit 11,27 $€$ im geschlossenen Ökozuchtprogramm.

In Abbildung 32 ist eine Veränderung der Rangierung der Zuchtprogramme bei verschiedenen unterstellten genetischen Korrelationen zu erkennen. Mit kleiner werdenden genetischen Korrelationen nehmen die naturalen Zuchtfortschritte der offenen Zuchtprogramme ab und sinken unterhalb des naturalen Zuchtfortschrittes des geschlossenen Ökozuchtprogramms. Das geschlossene Ökozuchtprogramm zeigt ab einer genetischen Korrelation von 0,9 die höchsten naturalen Zuchtfortschritte, gefolgt von dem Zuchtprogramm mit $40 \%$ konventionellem Genfluss. 


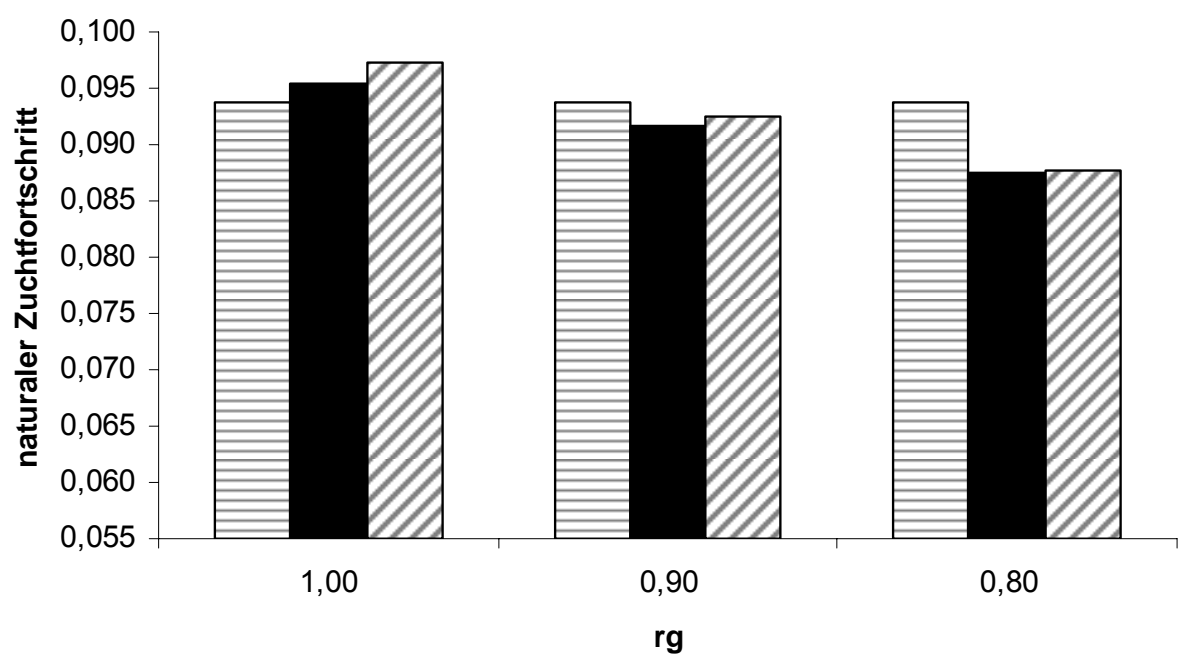

曰 $100 \%$ Öko — 80 \% Öko q 60 \% Öko

Abbildung 32: Naturaler Zuchtfortschritt für das Merkmal Eiweiß-kg bei $\mathrm{h}^{2}=0,34$.

Abbildung 33 zeigt die Auswirkung der Genotyp-Umwelt-Interaktion für das Merkmal Eiweiß-kg bei einer niedrigeren Heritabilität von 0,25. Alle drei untersuchten Parameter zeigen die gleiche Entwicklung bei Veränderung der Genflussanteile und genetischen Korrelationen wie bereits zu Abbildung 31 beschrieben.

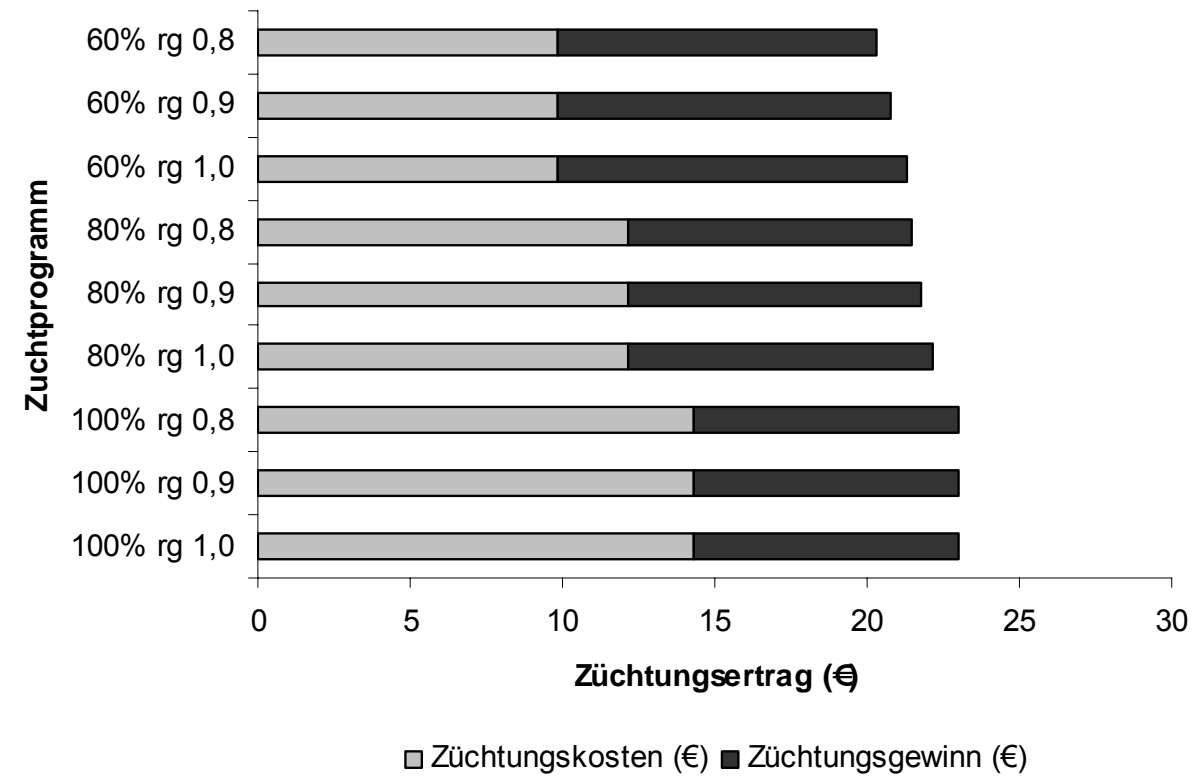

Abbildung 33: Züchtungsertrag (€) für das Merkmal Eiweiß-kg in unterschiedlichen Zuchtprogrammen mit verschiedenen genetischen Korrelationen $(\mathrm{rg})$ und $h^{2}=0,25$. 
Mit Ausnahme der gleich gebliebenen Züchtungskosten haben sich der Züchtungsertrag und der Züchtungsgewinn um insgesamt $3 €$ verringert. Ergänzend zu Abbildung 33 ist in Abbildung 34 der naturale Zuchtfortschritt für das Merkmal Eiweiß-kg bei einer unterstellten Heritabilität von 0,25 in allen drei Ökozuchtprogrammen dargestellt. Auch hier ist die Rangierung der naturalen Zuchtfortschritte mit den Ergebnissen der naturalen Zuchtfortschritte bei einer Heritabilität von 0,34 zu vergleichen. Aufgrund der geringeren Heritabilität erreichen die naturalen Zuchtfortschritte auch insgesamt ein niedriges Werteniveau.

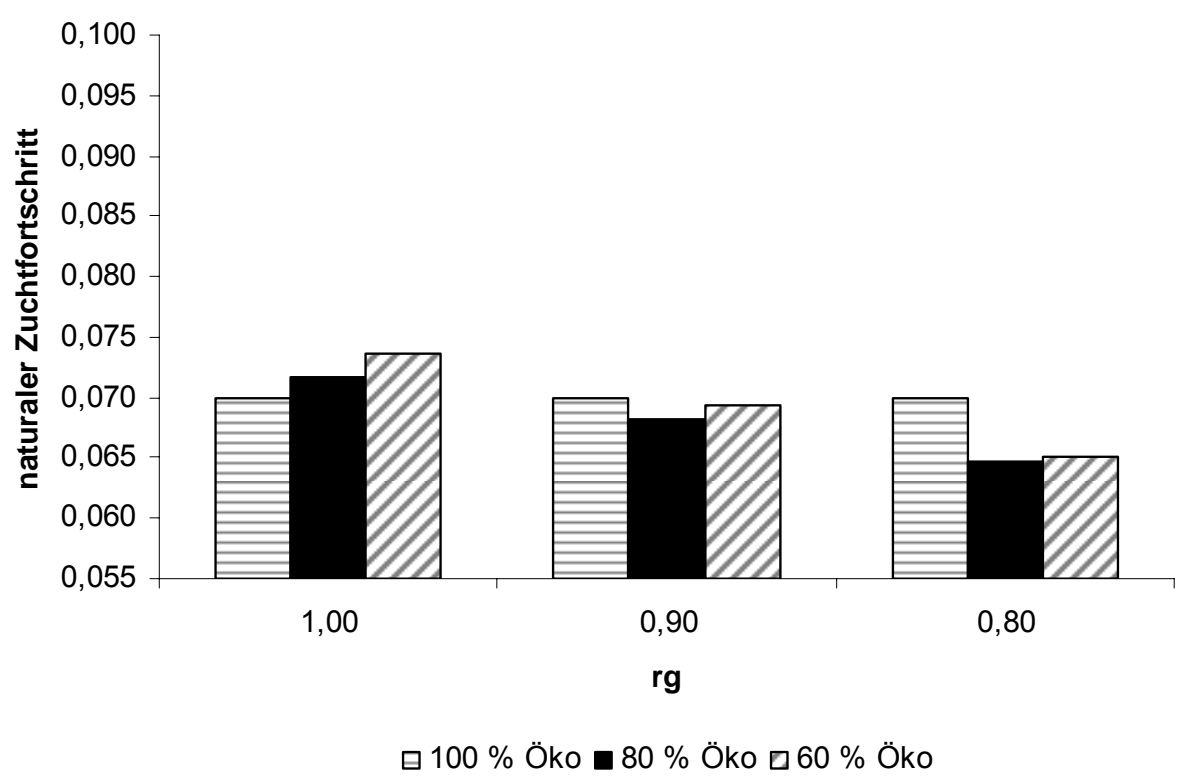

Abbildung 34: Naturaler Zuchtfortschritt für das Merkmal Eiweiß-kg bei $h^{2}=0,25$.

Auch diese Untersuchung zeigt, dass sich die modellierten Ökozuchtprogramme unter den verschiedenen vorausgesetzten Bedingungen kaum unterscheiden. Die naturalen Zuchtfortschritte variieren in einem Wertebereich zwischen 0,073 und 0,069.

\subsubsection{Genotyp-Umwelt-Interaktionen beim Merkmal Zellzahl}

Im Anschluss an die Auswertung der Genotyp-Umwelt-Interaktion für das Leistungsmerkmal Eiweißmenge werden in den folgenden zwei Kapiteln Untersuchungen zu GenotypUmwelt-Interaktionen für funktionale Merkmale am Beispiel der Zellzahl und der Nutzungsdauer durchgeführt. Beiden Merkmalen wurde eine Ausgangsheritabilität von 0,12 zugeordnet. In weiteren Vergleichen wurde die Heritabilität beider Merkmale auf 0,08 verkleinert und auf der anderen Seite auf 0,25 erhöht. Ein weiterer Unterschied sind die untersuchten genetischen Korrelationen. Da diese bei funktionalen Merkmalen grundsätzlich 
geringer sind als bei Leistungsmerkmalen, wurden in dieser Untersuchung die genetischen Korrelationen von 1,0 bis 0,2 in gleichmäßigen Schrittweiten von 0,2 variiert.

Auch bei einer Heritabilität von 0,12 und verschiedenen genetischen Korrelationen für das Merkmal Zellzahl ist bei Zunahme des konventionellen Genflusses insgesamt ein Rückgang im Züchtungsertrages von 25,13€ auf 21,65€ zu erkennen (Abbildung 35). Da sich die Auswirkungen der Parameter ausschließlich auf die Ökopopulation beziehen, lassen sich wie bereits zum Merkmal Eiweißmenge gleiche Schlüsse ziehen. Insofern ist der Züchtungsertrag in einem geschlossenen Ökozuchtprogramm am höchsten, da mit zunehmendem konventionellem Genfluss weniger Nachkommen im Ökozuchtprogramm zur Verfügung stehen und damit auch nur wenig Züchtungsertrag realisiert werden kann. Die geringeren Züchtungskosten für die Ökopopulation in den offenen Zuchtprogrammen sind darauf zurückzuführen, dass die Population kleiner wird und dadurch auch weniger Aufwand für das Testen von Bullen betrieben werden muss. Durch die geringen Kosten steigt auch der Züchtungsgewinn an. Im geschlossenen Programm belaufen sich die Kosten auf 14,27 $€$, im offenen Programm mit $20 \%$ Genfluss auf 12,18 $€$ und im Programm mit $40 \%$ Genfluss auf 9,84€. Der Züchtungsgewinn und der Züchtungsertrag nehmen auch innerhalb eines Zuchtprogramms ab, wenn die genetischen Korrelationen geringer werden, da der zunehmende Genanteil konventioneller Bullen keine Bedeutung für die ÖkoZuchtpopulation hat.

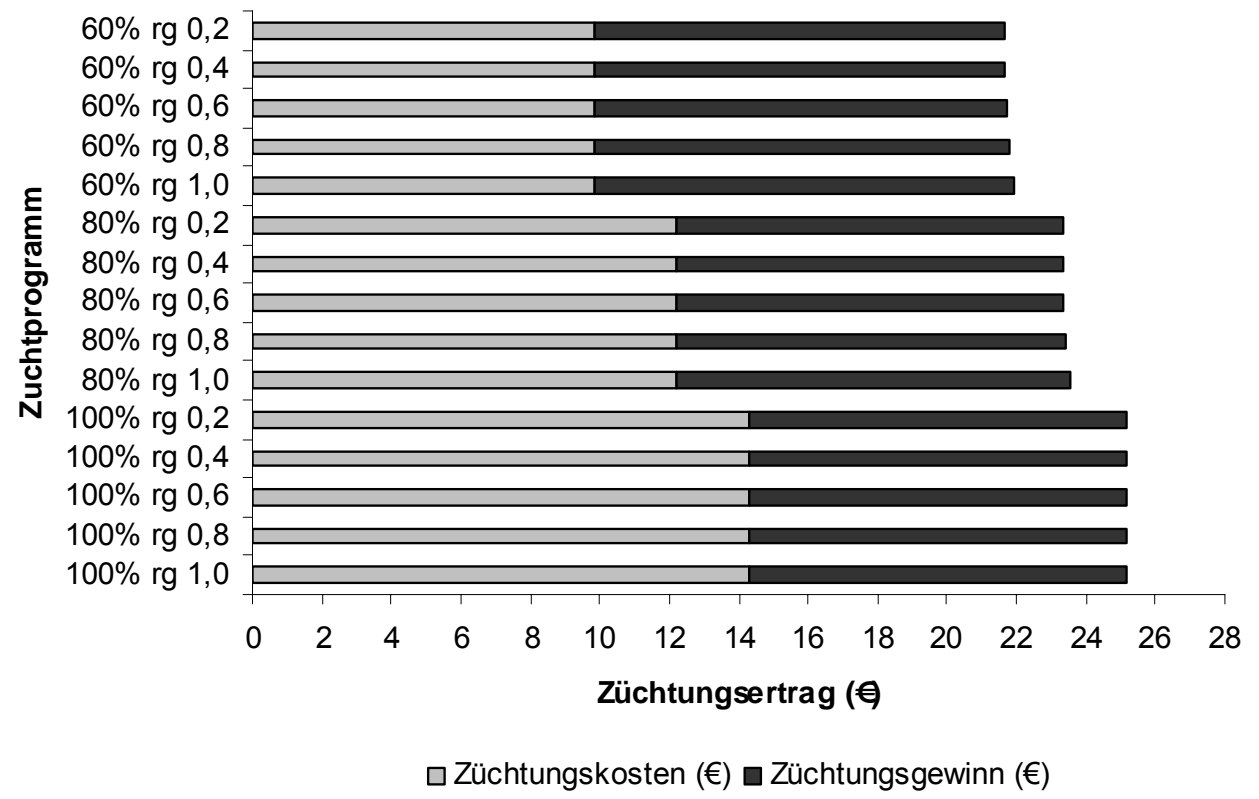

Abbildung 35: Züchtungsertrag (€) für das Merkmal Zellzahl in unterschiedlichen Zuchtprogrammen mit verschiedenen genetischen Korrelationen ( $\mathrm{rg}$ ) und $\mathrm{h}^{2}=0,12$. 
Bei Betrachtung der naturalen Zuchtfortschritte in Abbildung 36 ist auch in diesem Merkmal eine ähnliche Rangierung der Zuchtprogramme zu erkennen. Der naturale Zuchtfortschritt im geschlossenen Ökozuchtprogramm bleibt bei abnehmenden genetischen Korrelationen konstant bei -0,038. Das Zuchtprogramm mit $20 \%$ konventionellem Genfluss erreicht im Vergleich zum offenen Zuchtprogramm mit $40 \%$ konventionellem Genfluss schlechtere Werteniveaus. Insgesamt ist zu erkennen, dass sich neben dem geschlossenen Ökozuchtprogramm auch das zu $40 \%$ geöffnete Zuchtprogramm zur Verbesserung des Merkmals Zellzahl als vorteilhaft erweist.

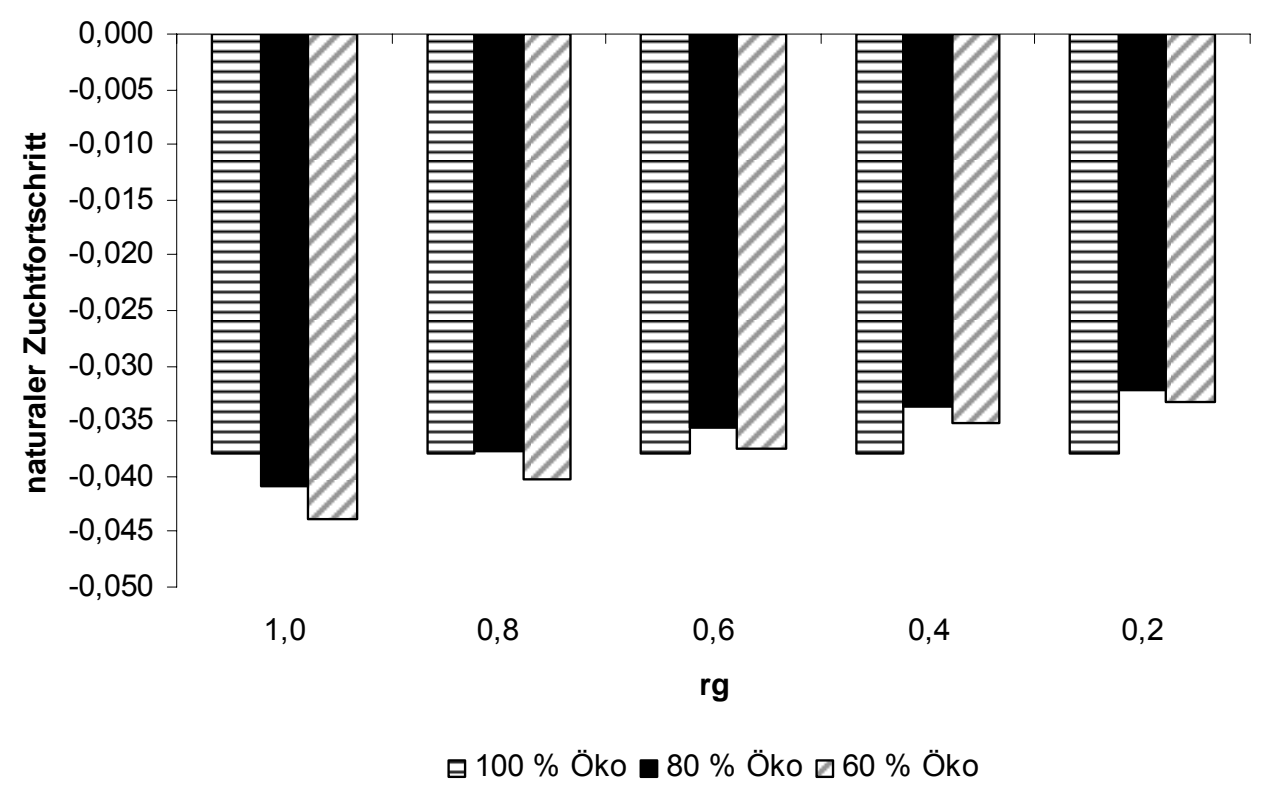

Abbildung 36: Naturaler Zuchtfortschritt für das Merkmal Zellzahl bei $h^{2}=0,12$.

Abbildung 37 zeigt die Untersuchung zur Genotyp-Umwelt-Interaktion beim Merkmal Zellzahl bei einer verringerten Heritabilität von 0,08. Auch unter diesen Umständen zeigen alle drei Zuchtprogramme einen abnehmenden Züchtungsertrag, wenn der konventionelle Einfluss erhöht wird. Im geschlossenen Ökozuchtprogramm liegt der Ertrag demnach bei $24,96 €$ und im offenen Zuchtprogramm mit $40 \%$ konventionellem Genfluss bei minimal $21,72 €$. Beide Werte sind aufgrund der herabgesetzten Heritabilität für das Merkmal minimal geringer als bei der vorigen Untersuchung. Die Züchtungskosten bleiben gleich und auch der Züchtungsgewinn deckt sich annähernd mit den Beträgen, die mit einer Heritabilität von 0,12 erreicht wurden. 


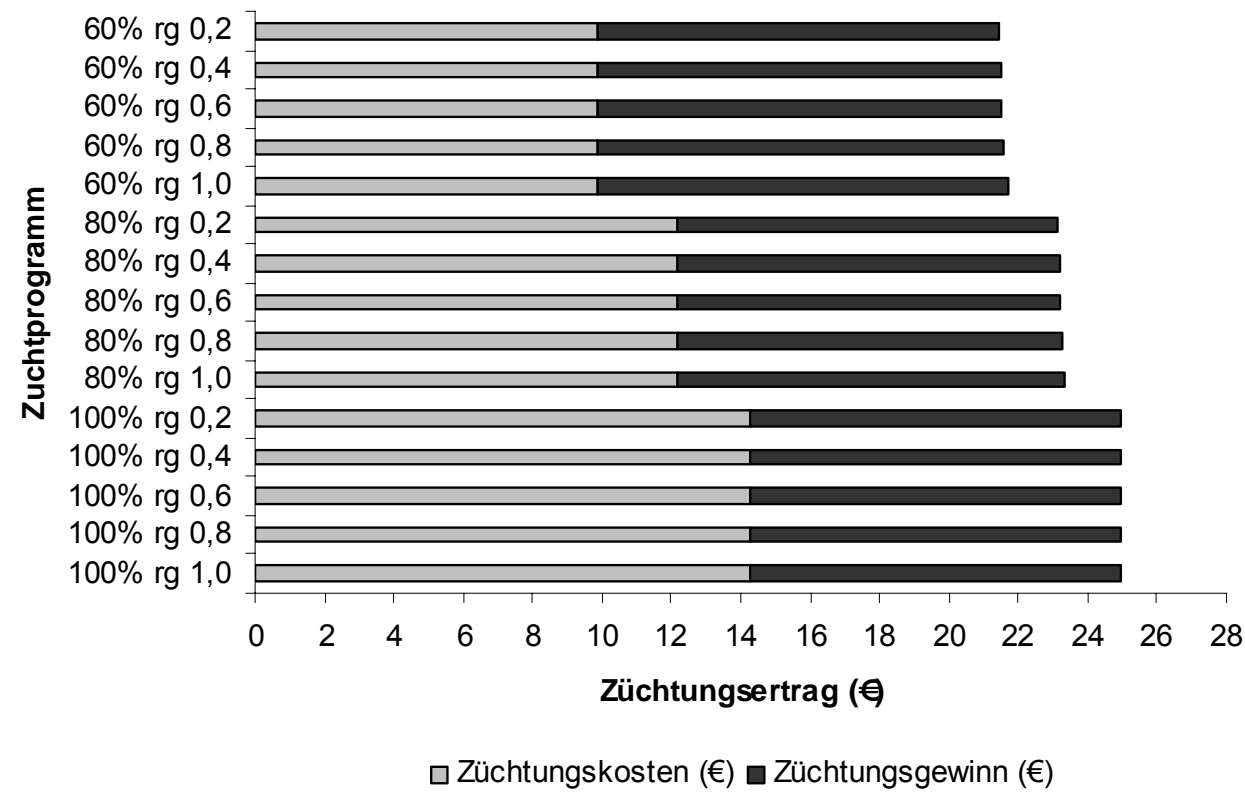

Abbildung 37: Züchtungsertrag (€) für das Merkmal Zellzahl in unterschiedlichen Zuchtprogrammen mit verschiedenen genetischen Korrelationen ( $\mathrm{rg}$ ) und $\mathrm{h}^{2}=0,08$.

In Abbildung 38 werden die naturalen Zuchtfortschritte für das Merkmal Zellzahl und einer Heritabilität von 0,08 dargestellt. Allgemein ist hier eine identische Entwicklung wie beim naturalen Zuchtfortschritt bei einer Heritabilität von 0,12 festzustellen. Bei einer genetischen Korrelation von 1,0 zeigen die offenen Zuchtprogramme bessere naturale Zuchtfortschritte für das Merkmal Zellzahl.

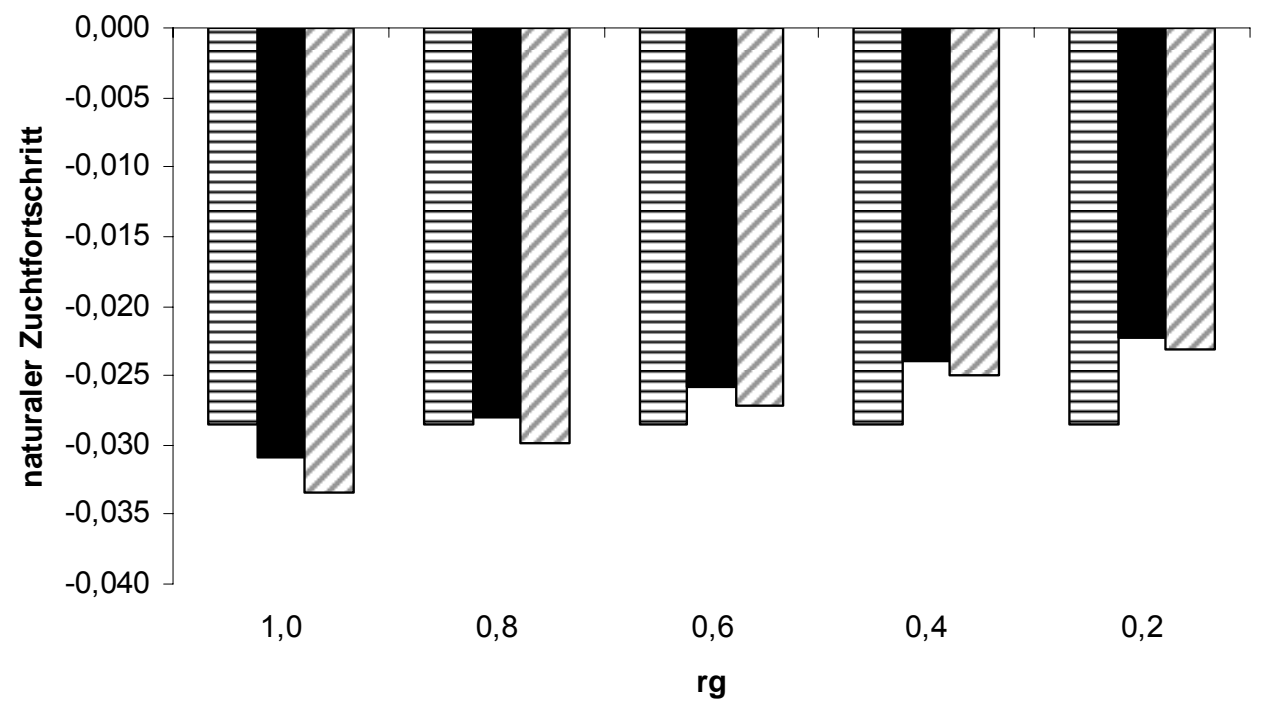

日 $100 \%$ Öko 80 \% Öko 60 \% Öko

Abbildung 38: Naturaler Zuchtfortschritt für das Merkmal Zellzahl bei $h^{2}=0,08$. 
Mit Verringerung der genetischen Korrelation verändert sich jedoch diese Rangierung und ab einer genetischen Korrelation von 0,6 zeigt das geschlossene Ökozuchtprogramm mit einem konstanten Wert von -0,028 den besten naturalen Zuchtfortschritt. Aber auch mit einem offenen Zuchtprogramm mit $40 \%$ konventionellem Genanteil werden bessere naturale Zuchtfortschritte erreicht als bei einem offenen Zuchtprogramm mit nur $20 \%$ konventionellem Genanteil. Zum Schluss dieser Untersuchung wurde für das Merkmal Zellzahl auch eine höhere Heritabilität von 0,25 angenommen (Abbildung 39). Die Entwicklung der Ergebnisse sind abgesehen von höheren Werteniveaus mit den Basisergebnissen und denen bei Verringerung der Heritabilität zu vergleichen.

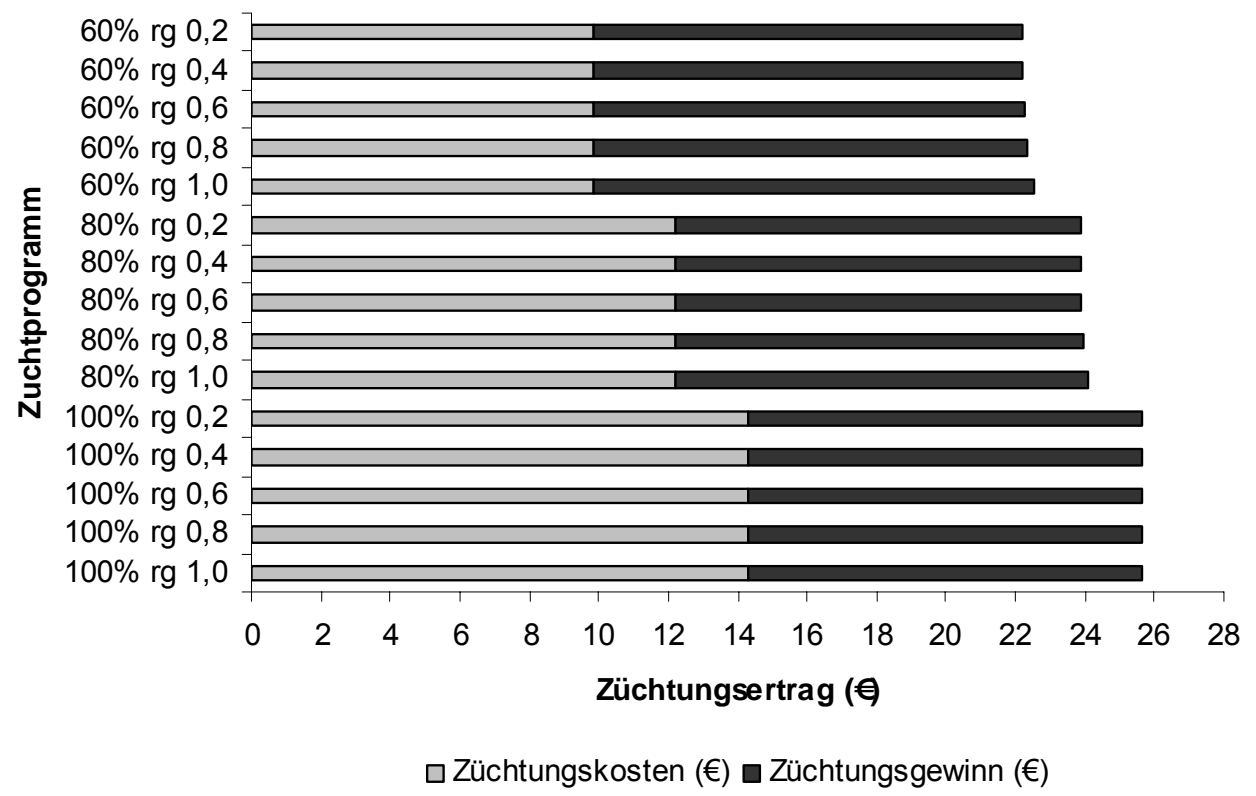

Abbildung 39: Züchtungsertrag (€) für das Merkmal Zellzahl in unterschiedlichen Zuchtprogrammen mit verschiedenen genetischen Korrelationen $(\mathrm{rg})$ und $h^{2}=0,25$.

Neben den in allen Varianten gleich gebliebenen Kosten erhöht sich der Züchtungsgewinn von $11,37 €$ beim $100 \%$ Ökozuchtprogramm auf $11,90 €$ beim $80 \%$ Ökozuchtprogramm und schließlich auf $12,69 €$ Gewinn bei einer unterstellten genetischen Korrelation von 1,0. Eine gegenläufige Entwicklung ist im Züchtungsertrag zu erkennen. Dieser verringert sich mit Zunahme des konventionellen Genflusses von 25,64€, auf 24,09€ und schließlich auf $22,54 €$. Der Züchtungsertrag wird durch die Verringerung der Ökopopulation und der damit einhergehenden Verringerung der Kühe, die für Testbesamungen zur Verfügung stehen negativ beeinflusst. Somit lässt sich auch der Rückgang der Züchtungskosten in der Ökopopulation erklären. 


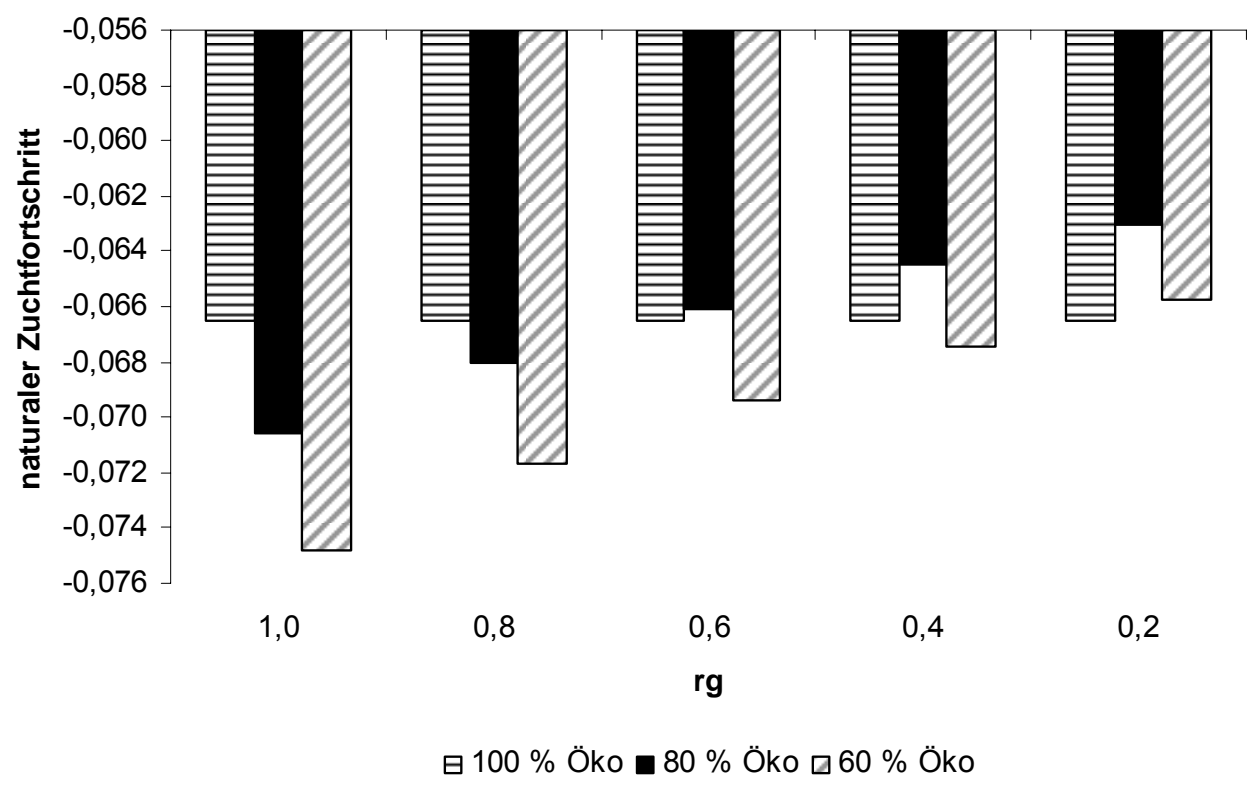

Abbildung 40: Naturaler Zuchtfortschritt für das Merkmal Zellzahl bei $h^{2}=0,25$.

Abbildung 40 zeigt abschließend den naturalen Zuchtfortschritt für das Merkmal Zellzahl bei einer Heritabilität von 0,25. Auffällig ist hier, dass alle Werte im Vergleich zu den anderen Heritabilitäten, deutlich besser geworden sind. Der naturale Zuchtfortschritt für das geschlossene Ökozuchtprogramm beträgt in dieser Untersuchung konstant -0,066. Die naturalen Zuchtfortschritte der offenen Zuchtprogramme nehmen mit Verringerung der genetischen Korrelation kontinuierlich ab. Anders als bei den vorigen Ergebnissen werden mit dem offenen Zuchtprogramm mit $40 \%$ konventionellem Genfluss bis zu einer genetischen Korrelation von 0,4 die besten naturalen Zuchtfortschritte erreicht. Erst ab einer genetischen Korrelation von 0,2 kommt es zur Umrangierung zwischen dem zu 40 \% geöffneten Zuchtprogramm und dem geschlossenen Zuchtprogramm.

Auch das Zuchtprogramm mit $20 \%$ konventionellem Genfluss realisiert bis zu einer genetischen Korrelation von 0,8 mehr naturalen Zuchtfortschritt als das geschlossene Zuchtprogramm. Insgesamt zeigt das zu $20 \%$ geöffnete Zuchtprogramm aber die niedrigsten naturalen Zuchtfortschritte. Der beste naturale Zuchtfortschritt von -0,074 wird schließlich im offenen Zuchtprogramm mit $40 \%$ konventionellem Genanteil bei einer genetischen Korrelation von 1,0 erreicht. 


\subsubsection{Genotyp-Umwelt-Interaktionen beim Merkmal Nutzungsdauer}

Als weiteres Merkmal zur Untersuchung der Auswirkungen von Genotyp-UmweltInteraktionen bei funktionalen Merkmalen wurde die Nutzungsdauer untersucht. Auch dieses Merkmal wurde in einem geschlossenen Ökozuchtprogramm und zwei Programmen mit unterschiedlich starkem konventionellem Genfluss untersucht. Nach den Basisdurchläufen wurde die Heritabilität von 0,12 auf 0,08 und 0,25 verändert und in allen Programmen untersucht.

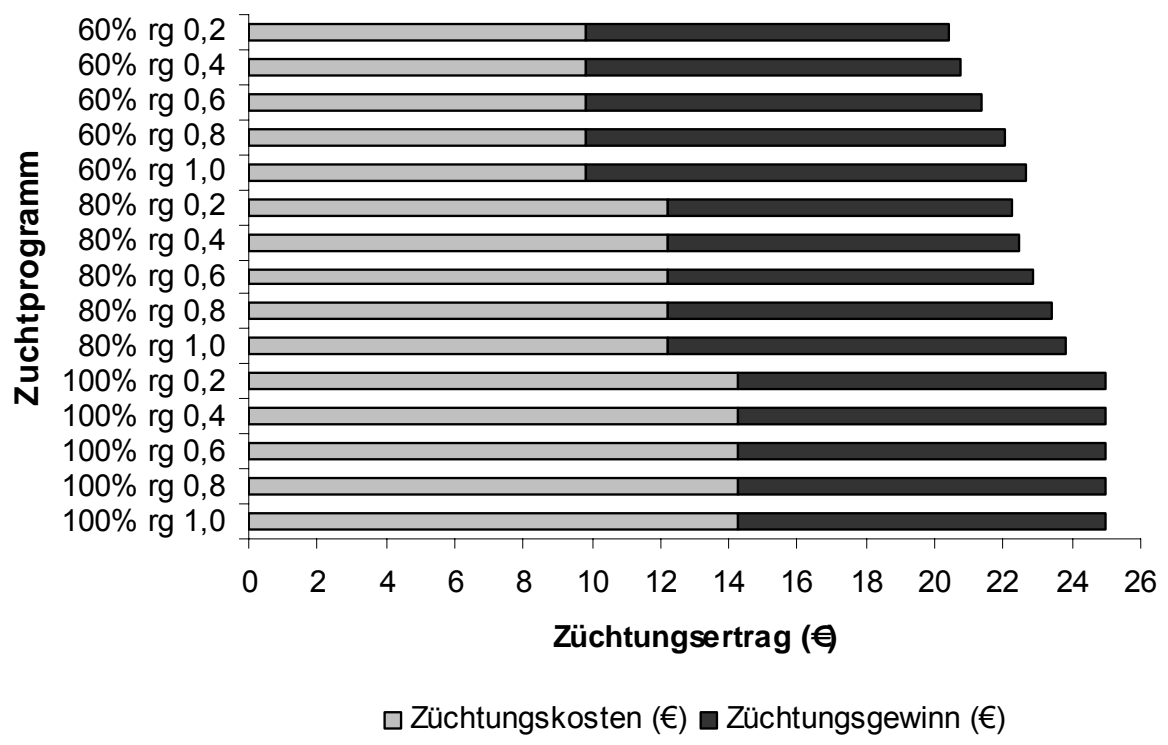

Abbildung 41: Züchtungsertrag (€) für das Merkmal Nutzungsdauer in unterschiedlichen Zuchtprogrammen mit verschiedenen genetischen Korrelationen $(\mathrm{rg})$ und $\mathrm{h}^{2}=0,12$.

Abbildung 41 zeigt den Züchtungsertrag nach dem Basisdurchlauf zur Untersuchung der Genotyp-Umwelt-Interaktion beim Merkmal Nutzungsdauer. Obwohl die Entwicklungen der Ergebnisse von gleichen Faktoren abhängen wie bereits zum Merkmal Zellzahl beschrieben wurde, fällt hier auf, dass in einem offenen Ökozuchtprogramm mit $40 \%$ konventionellem Genfluss und einer genetischen Korrelation von 1,0 mehr Züchtungsertrag realisiert wird, als in einem offenen Zuchtprogramm mit $20 \%$ konventionellem Genfluss und gleicher genetischer Korrelation. Werden die genetischen Korrelationen innerhalb dieses Zuchtprogramms kleiner, nehmen auch die Züchtungserträge wie erwartet ab.

Der höchste Züchtungsertrag wird im geschlossenen Ökozuchtprogramm erreicht und beträgt $24,99 €$. Beim Züchtungsgewinn liegt der Maximalwert bei $12,78 €$ und wird im offenen Zuchtprogramm mit 40 \% konventionellem Genfluss bei einer genetischen Korrelation von 1,0 erreicht. Die Züchtungskosten verringern sich mit einer kleiner werdenden Ökopopulation von $14,27 €$ auf $12,18 €$ und $9,84 €$. 
Abbildung 42 zeigt den naturalen Zuchtfortschritt für das Merkmal Nutzungsdauer bei einer Heritabilität von 0,12 in allen drei ökologischen Zuchtprogrammen. Am Verlauf der Balken ist klar zu erkennen, dass die offenen Ökozuchtprogramme bei einer genetischen Korrelation $<1,0$ immer weniger naturalen Zuchtfortschritt erreichen. Bei einer genetischen Korrelation von 1,0 liegen die offenen Programme mit naturalen Zuchtfortschritten von 0,073 bei $20 \%$ und 0,075 bei $40 \%$ konventionellem Einfluss über dem geschlossenen Ökozuchtprogramm. Bis zu einer genetischen Korrelation von 0,2 nehmen die naturalen Zuchtfortschritte nur langsam ab. Das geschlossene Ökozuchtprogramm zeigt unbeeinflusst von den genetischen Korrelationen einen Wert von 0,07.

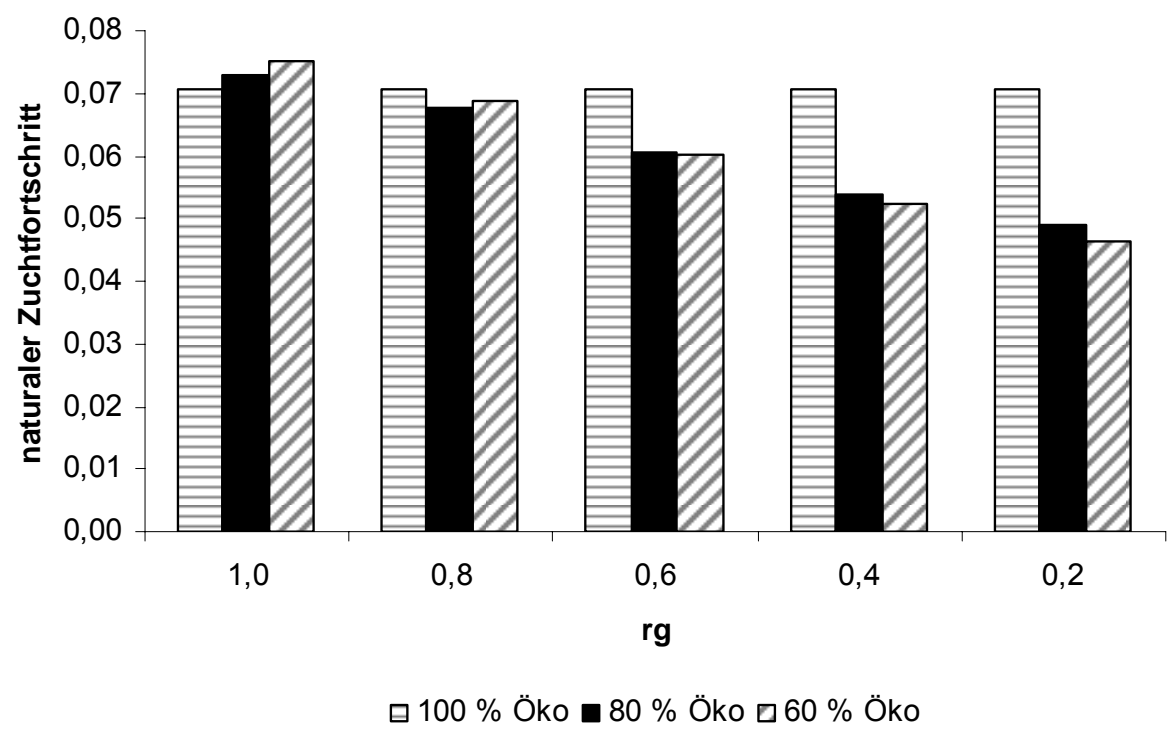

Abbildung 42: Naturaler Zuchtfortschritt für das Merkmal Nutzungsdauer bei $h^{2}=0,12$.

In Abbildung 43 ist der Züchtungsertrag für die Nutzungsdauer bei einer niedrigeren Heritabilität von 0,08 dargestellt. Auch hier geht der Züchtungsertrag sowohl mit zunehmendem konventionellen Einfluss, als auch bei kleineren genetischen Korrelationen innerhalb eines Zuchtprogramms zurück. Der Züchtungsgewinn steigt eher leicht an, je mehr konventioneller Einfluss vorherrscht. Innerhalb eines Zuchtprogramms geht dieser aber auch zurück, wenn die genetischen Korrelationen kleiner werden, da der ansteigende Anteil konventioneller Bullen keinen Einfluss auf den Züchtungsgewinn in der Ökopopulation hat. Der meiste Züchtungsgewinn liegt schließlich bei 10,89€ und wird im offenen Ökozuchtprogramm mit $40 \%$ konventionellem Genfluss und bei einer rg von 1,0 erreicht. Anders als bei den bisher untersuchten Merkmalen, erzielt das offene Ökozuchtprogramm mit $20 \%$ Genanteil ab einer genetischen Korrelation kleiner als 0,6 höhere naturale Zuchtfortschritte als das offene Zuchtprogramm mit $40 \%$ konventionellem Genanteil. 


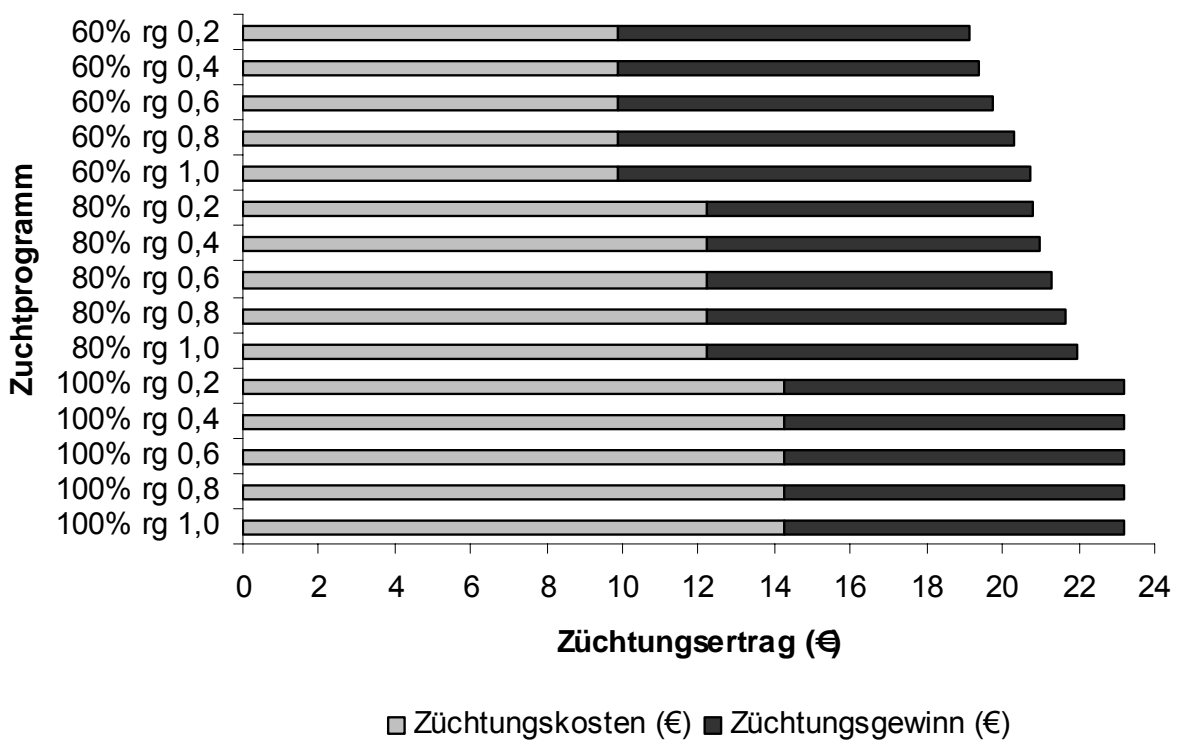

Abbildung 43: Züchtungsertrag (€) für das Merkmal Nutzungsdauer in unterschiedlichen Zuchtprogrammen mit verschiedenen genetischen Korrelationen (rg) und $h^{2}=0,08$.

Insgesamt nehmen die Züchtungserträge und auch die in Abbildung 44 dargestellten naturalen Zuchtfortschritte aufgrund der niedrigeren Heritabilität niedrigere Werteniveaus ein. Das geschlossene Ökozuchtprogramm zeigt einen konstanten naturalen Zuchtfortschritt von 0,048. Die anfängliche Dominanz der offenen Programme mit einem Zuchtfortschritt von jeweils 0,05 geht bis zu einer genetischen Korrelation von 0,2 schrittweise zurück, wodurch es zu einer Verschiebung in der Rangierung der Zuchtprogramme kommt.

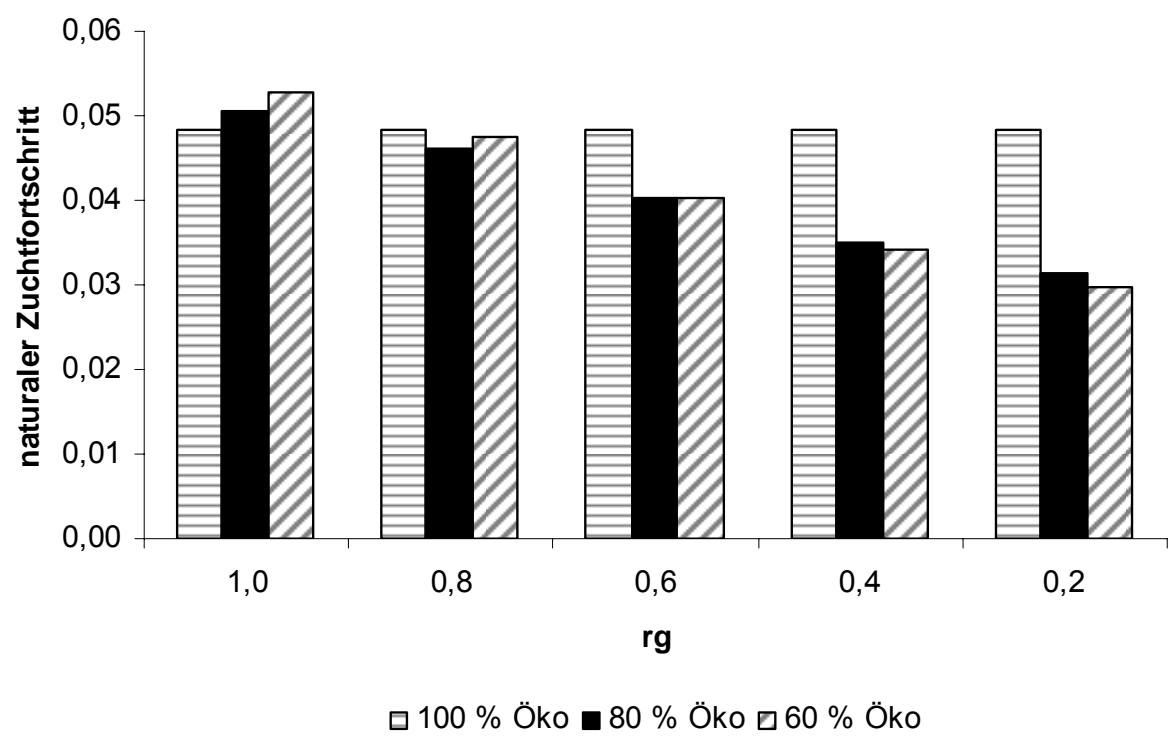

Abbildung 44: Naturaler Zuchtfortschritt für das Merkmal Nutzungsdauer bei $h^{2}=0,08$. 
Als letzte Alternative für die Heritabilität der Nutzungsdauer ist in Abbildung 45 der Züchtungsertrag bei einer unterstellten Heritabilität von 0,25 abgebildet. Insgesamt betrachtet zeigen sich hier die höchsten Züchtungserträge und Züchtungsgewinne. Der höchste Züchtungsertrag liegt bei $30,83 €$ und wird im geschlossenen Ökozuchtprogramm mit der größten Ökopopulation erreicht. Wie bereits bei einer Heritabilität von 0,12 festgestellt, fällt auch hier der Züchtungsertrag bei einem offenen Zuchtprogramm mit $40 \%$ konventionellem Genfluss auf. Wird die genetische Korrelation innerhalb eines Programms verringert, hat dies wiederum eine Reduzierung der Züchtungserträge und der Züchtungsgewinne zu Folge.

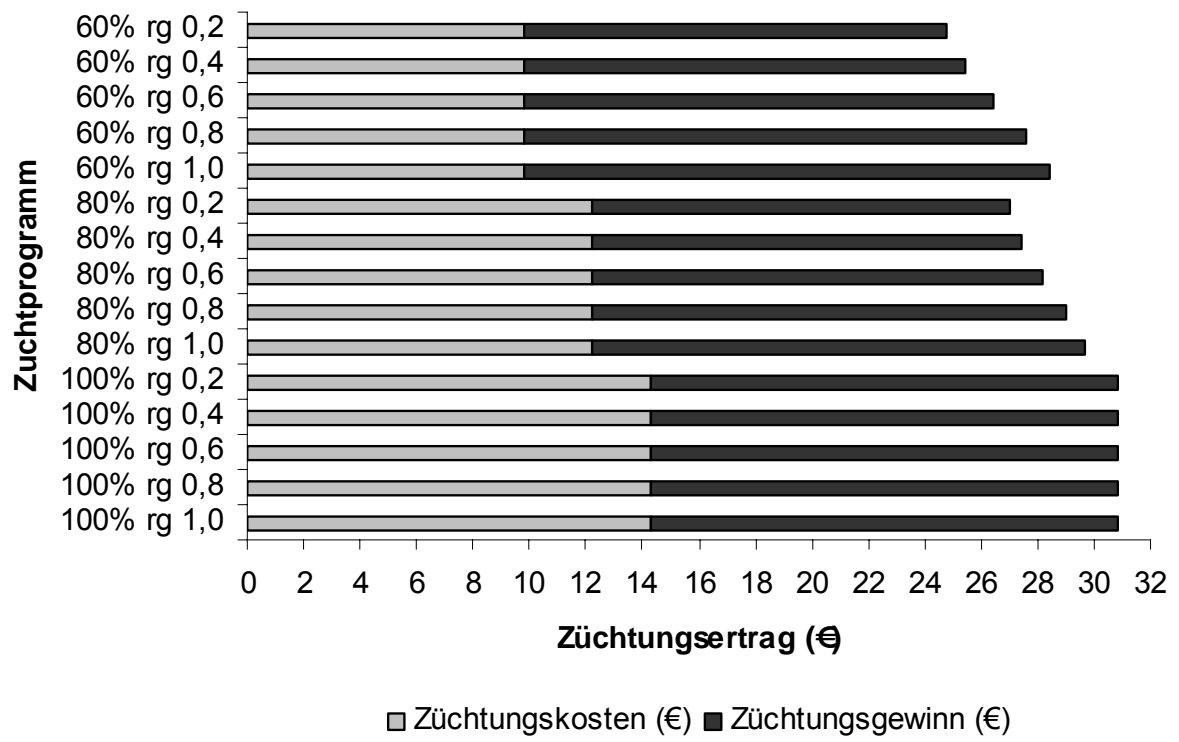

Abbildung 45: Züchtungsertrag (€) für das Merkmal Nutzungsdauer in unterschiedlichen Zuchtprogrammen mit verschiedenen genetischen Korrelationen (rg) und $\mathrm{h}^{2}=0,25$.

Die letzte Abbildung dieser Auswertung zeigt den naturalen Zuchtfortschritt für das Merkmal Nutzungsdauer und einer erhöhten Heritabilität von 0,25 (Abbildung 46). Auch hier liegt das Niveau deutlich höher als bei niedrigeren Heritabilitäten. Der naturale Zuchtfortschritt im geschlossenen Ökozuchtprogramm liegt bei 0,134. Im Vergleich zu einer Heritabilität von 0,12 hat sich dieser Wert verdoppelt. Die offenen Zuchtprogramme zeigen bei geringer werdenden genetischen Korrelationen dieselben rückläufigen Entwicklungen wie bei den alternativ eingesetzten Heritabilitäten. 


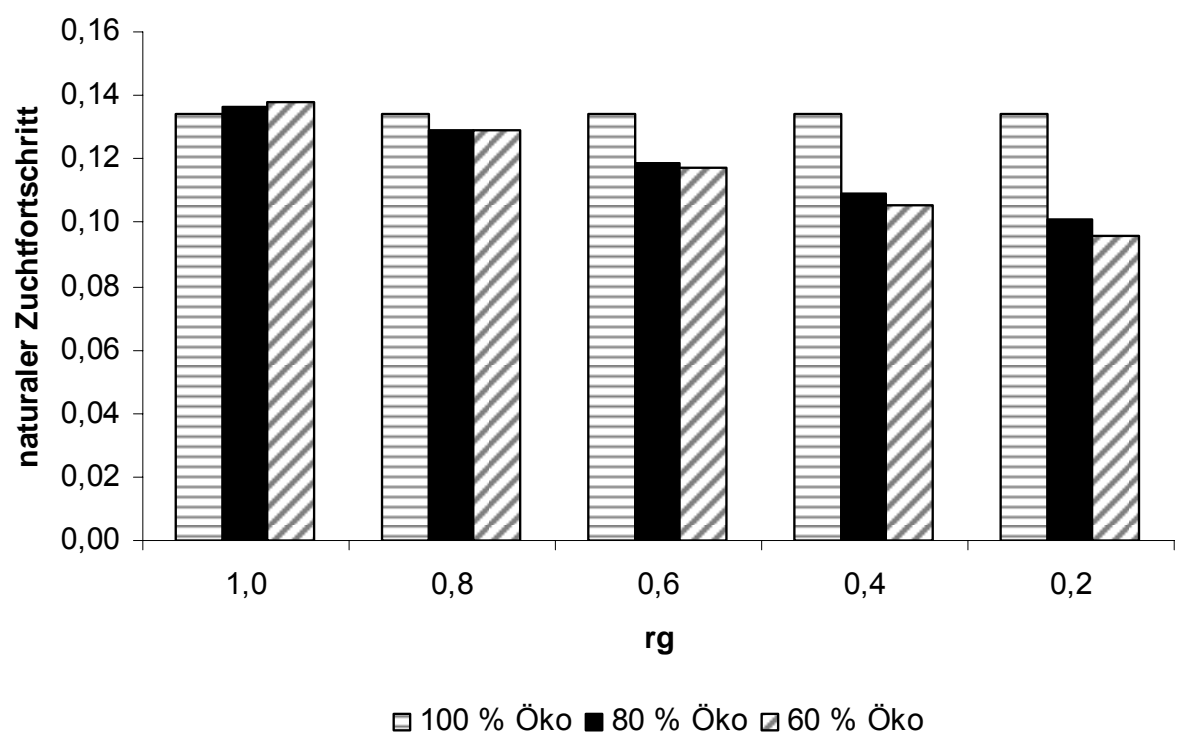

Abbildung 46: Naturaler Zuchtfortschritt für das Merkmal Nutzungsdauer bei $h^{2}=0,25$. 


\section{Diskussion}

In der vorliegenden Arbeit werden auf Grundlage von Daten der beim VIT identifizierten ökologischen Betriebe auf genetische und phänotypische Trends für Leistungs- und funktionale Merkmale erhoben und mittels konventioneller Vergleichsdaten bewertet. Des Weiteren wird in der Studie ein Fokus auf die mit Hilfe des Computerprogramms ZPLAN durchgeführten Untersuchungen gelegt, um die Effizienz eines ökologischen Zuchtprogramms und verschiedene Zuchtstrategien bewerten zu können. In den folgenden Abschnitten werden die wesentlichen Untersuchungsergebnisse im Kontext zu bereits durchgeführten Forschungsvorhaben zu diesem Thema diskutiert. Durch die Auswertung der Literatur und der eigenen Ergebnisse soll beurteilt werden, inwieweit ein eigenes Zuchtprogramm speziell für ökologisch wirtschaftende Betriebe sinnvoll erscheint bzw. wie sich einzelne Zuchtstrategien bewähren.

\subsection{Genetische und phänotypische Trends für Leistungsmerkmale und funktionale Merkmale}

Aufgrund von wenigen vorhandenen wissenschaftlichen Studien und Veröffentlichungen über die Schätzung der genetischen Trends in nationalen Kuhpopulationen (Swalve und Höver, 2003), werden die hier vorliegenden Ergebnisse teilweise mit internationalen Forschungsergebnissen diskutiert. Hierbei müssen jedoch die nach König und Swalve (2000) vorherrschenden länderspezifischen Unterschiede im absoluten genetischen Niveau mitberücksichtigt werden. Da insbesondere Vergleichsstudien zu den Trends auf ökologischen Milchviehbetrieben in Deutschland fehlen, kann der Trend bei den Bullen ausschließlich in Anlehnung an konventionelle Verhältnisse diskutiert werden. Zu den Trends bei ökologischen Kühen, v. a. zu phänotypischen Trends, liegen Vergleiche aus den Niederlanden und Kanada vor.

\subsubsection{Genetische Trends der Leistungsmerkmale bei Ökobullen und konventionel- len Bullen}

Der von van Tassel und van Vleck (1991), Swalve (1999), Anacker (2003), sowie von Fürst (2006) analysierte positive Trend der Bullen im Merkmal Milchmenge, zeigt sich auch in den eigenen Ergebnissen der Öko- und konventionellen Bullen. Werden die Regressionskoeffizienten beider Gruppen miteinander verglichen, lässt sich bei den konventionellen Bullen ein leicht höherer positiver Trend der Zuchtwerte Milch-, Fett- und Eiweißmenge erkennen. Das mehr auf funktionale Merkmale ausgerichtete ökologische Zucht- 
ziel könnte ein Indiz für die unterhalb des konventionellen Niveaus liegende Trendkurve sein. Neben den Berichten des BMELV (2007) bestätigen auch internationale Studien von Sato et al. (2004), Nauta et al. (2006a) und Rozzi et al (2007), dass die Leistungen ökologischer Milchviehbetriebe unter dem Leistungsniveau konventioneller Vergleichsbetriebe liegen. Eine Verbesserung im Management in den letzten Jahren, aber auch Veränderungen züchterischer Maßnahmen und die Situation auf dem Milchmarkt, haben diese deutliche Aufwärtsentwicklung der Milchleistung weltweit hervorgerufen (Fürst, 2006). Weiterhin spricht eine hohe Korrelation zwischen den Mengenmerkmalen Milch-, Fett- und Eiweiß$\mathrm{kg}$ für den vorliegenden positiven genetischen Trend.

Im Gegensatz zu den Mengenmerkmalen zeigen die Inhaltsstoffe keinen positiven Trend in den betrachteten Jahrgängen. Im Gegensatz zum Eiweißgehalt, dessen Entwicklung als marginal positiv zu betrachten ist, zeigt sich für den Fettgehalt ein negativer Trend. Swalve (1999), Anacker (2003) und Bergfeld (2004) sprechen von einem negativen Trend in den Merkmalen Eiweiß- und Fettgehalt. Die Diskrepanz zwischen der Milchmenge und den Inhaltsstoffen lässt sich genetisch durch die negative Korrelation zwischen diesen Merkmalen erklären. Schüler et al. (2001) geben genetische Korrelationen zwischen Milchmenge und Milcheiweißgehalt im Bereich von $-0,00$ bis $-0,65$ und zwischen Milchmenge und Milchfettgehalt im Bereich von 0,10 bis $-0,50$ an. Auch Fürst (2005) fand in Bezug auf Fleckvieh- und Braunviehbullen negative genetische Korrelationen zwischen der Milchmenge und den Gehaltsmerkmalen im Bereich von $-0,40$ bis $-0,50$. Zwischen der Eiweißmenge und dem Eiweißgehalt ermittelte Fürst (2005) zudem genetische Korrelationen im Bereich von $-0,09$ und $+0,02$. Durch diesen von ihm ermittelten neutralen bis leicht negativen Zusammenhang erklärt der Autor, dass eine Erhöhung der Eiweißmenge eine Erhöhung der Milchmenge bedingt, nicht aber eine Erhöhung des Gehalts an Eiweiß. Eine negative genetische Korrelation erschwert weiterhin den Erfolg der Selektion auf ein Merkmal (Fürst, 2006). Dieser Effekt erklärt die eigenen Entwicklungen für Fett- und Eiweißgehalt im Vergleich zur Milchmenge. Ergänzend zu diesen genetischen Grundlagen, ist nach Meinung von Swalve (1999) und Bergfeld (2004) der genetische Trend bei den Bullen auf die veränderte Zuchtpolitik und die Verschiebung des Selektionsschwerpunktes auf die Mengenmerkmale zurückzuführen. Bedingt durch die Steigerung der Mengenzuchtwerte lässt sich auch der in beiden Bullengruppen ermittelte positive Trend des Relativzuchtwerts Milchleistung (RZM) erklären. 


\subsubsection{Genetische Trends in den funktionalen Merkmale bei Ökobullen und konven- tionellen Bullen}

Da u. a. das Merkmal Fruchtbarkeit Bestandteil des Relativzuchtwertes Zuchtleistung ist, könnte der nach Fürst (2005) ermittelte Antagonismus zwischen der Milchmenge und der Fruchtbarkeit die Entwicklung in diesem Merkmal erklären. Da die Merkmale Non-ReturnRate, Kalbeverlauf und Totgeburten die Entwicklung des Relativzuchtwertes Zuchtleistung mitbestimmen und diese Trends sowohl positiv als auch negativ sind, hält sich der Trend für den RZZ entsprechend in der Waage bzw. bewegt sich aufgrund der Regressionskoeffizienten von $-0,07$ bei den Ökobullen und $+0,05$ bei den konventionellen Bullen über die Jahre auf einem annähernd gleichen Niveau. Aber auch niedrige Heritabilitäten der Fitness- und Fruchtbarkeitsmerkmale, schränken die Verbesserung des RZZ ein.

Der über die Jahre anhaltende negative Trend im Merkmal Relativzuchtwert Zellzahl bestätigt sich auch in der Arbeit von Bo (2004). Bo (2004) und Fürst (2005) machen auch hier deutlich, dass zum einen die niedrige Heritabilität die züchterische Entwicklung dieses Merkmals beeinträchtigt. Weiterhin ermittelten Swalve (1999) und auch Fürst (2005) Antagonismen zwischen Milch-Zuchtwerten und den Fitnessmerkmalen bzw. zwischen dem RZM und dem RZS sowie dem RZN, was als weitere Erklärung für die rückläufige Entwicklung der eigenen Untersuchung zum RZS dienen kann. Ein weiterer Beleg für einen negativen genetischen Trend im Merkmal Zellzahl ist, dass die Euterprobleme in den letzten 20 Jahren deutlich gestiegen sind (Brade, 2005).

Durch den bereits erwähnten Antagonismus lässt sich, zwar nicht ganz so deutlich, der Trend in der Nutzungsdauer bzw. im RZN ansatzweise interpretieren. Als weitere Erklärung dient auch die positive Entwicklung des RZE, denn obwohl Swalve (1999) auf die schwache Korrelation zwischen RZE und RZN hinweist, werden Exterieurmerkmale als Hilfsmerkmale zur frühen Abschätzung der Nutzungsdauer herangezogen (Swalve, 1999; Fürst, 2006). Demnach richtet sich der Trend für den RZN überwiegend nach dem Trend des RZE. Dessen positiver Anstieg lässt sich dadurch begründen, dass der RZE neben den Mengenmerkmalen und der Nutzungsdauer einen weiteren Selektionsschwerpunkt darstellt. Bergfeld (2004) erläutert anhand dieser Argumentation, dass die positive Entwicklung des RZE dazu dient, eine Verbesserung der Funktionalität der Milchrindproduktion zu erreichen. Die positive Veränderung im RZG erklärt sich vor allem durch die positiven Veränderungen von RZM und RZE, die einen großen Anteil am RZG ausmachen. 
Niedrige Heritabilitäten für die Merkmale Non-Return-Rate, Kalbeverlauf und Totgeburtenrate können die insgesamt negativen Entwicklungen erklären. Zu diesem Schluss kommt auch Fürst (2005). Er erläutert, dass geringe Heritabilitäten die züchterischen Möglichkeiten zur Verbesserung der Fitnessmerkmale einschränken, was sich sehr gut anhand der dargestellten Regressionskoeffizienten widerspiegelt. Eine Optimierung des Erfolgs in diesen Merkmalen ist nach Bergfeld (2004) und Fürst (2005) nur durch entsprechende Veränderungen der Umweltbedingungen möglich.

Insgesamt zeigen die fast parallel laufenden Kurven und geringen Unterschiede in den Regressionskoeffizienten in allen Untersuchungen, dass die eingesetzten Bullen unter verschiedenen Umweltbedingungen annähernd die gleichen Entwicklungen in einzelnen Merkmalen realisieren können.

\subsubsection{Genetische Trends bei Ökokühen}

Anhand der Zuchtwerte der Kühe lassen sich bezüglich der Merkmale Milch-, Fett- und Eiweißmenge gleiche genetische Trends erkennen, die auch bei den Bullen beobachtet werden konnten. Somit ist der bei den Kühen zu beobachtende Anstieg der Mengenmerkmale (vgl. auch Swalve, 1999; Anacker, 2003; Swalve und Höver, 2003; Nauta et al., 2006a) und der Rückgang der Inhaltsstoffe (Swalve und Höver, 2003) eine Funktion des Zuchtziels, aber gleichermaßen ein aufgrund der negativen Korrelationen zwischen der Milchmenge und den Gehaltsmerkmalen genetisch bedingter Prozess (Schüler et al., 2001; Fürst, 2005).

Der im Vergleich zum Fettgehalt nicht ganz so stark negativ verlaufende Trend für Eiweißgehalt und das leicht höhere Niveau der Eiweißmenge im Vergleich zur Fettmenge, sind vermutlich auf das für die Berechnung des RZM definierte Fett-/Eiweißverhältnis von 1:4 zurückzuführen. Auffällig ist auch der leicht positive Anstieg des Eiweißgehalts ab dem Geburtsjahr 2002, was als Reaktion auf die seit dem gleichen Jahr geltende Einbeziehung der Zuchtwerte für den Fett- und Eiweißgehalt zur Berechnung des RZM erklärt werden kann. Wie auch Swalve und Höver (2003) berichten, liegt der Schwerpunkt zur Berechnung des RZM in der Eiweißmenge. Demzufolge lässt sich durch die vorliegende Steigung der Eiweißmenge auch der positive Trend im RZM erklären. Der Trend des RZG wird hauptsächlich durch den RZM bestimmt, welcher im RZG eine relative Gewichtung von $50 \%$ besitzt. 
Da sich die Relativzuchtwerte Exterieur, Zellzahl, Nutzungsdauer und Zuchtleistung bei den Ökokühen allgemein positiv entwickeln, könnten diese Trends auf das stärker auf funktionale Merkmale ausgerichtete ökologische Zuchtziel zurückzuführen sein. Bezogen auf alle Milchviehbetriebe berichtet Bünger (1999) davon, dass die Merkmale des Exterieurs für die Wirtschaftlichkeit eines Betriebes immer mehr an Bedeutung gewinnen. Dieser Wandel kann schließlich der Auslöser für den in der eigenen Untersuchung gefundenen deutlich positiven Trend im Merkmal RZE sein.

Zur Schätzung des RZN, wird, wie auch Swalve (1999) und Fürst (2006) beschreiben, auf den RZE zurückgegriffen. Auch Bünger (1999) hebt in diesem Zusammenhang den signifikanten Einfluss der Exterieurmerkmale aller eingetragenen Kühe auf die Nutzungsdauer hervor. Schließlich werden zur indirekten Erhöhung der Nutzungsdauerzuchtwerte von jungen Bullen korrelierte Exterieurmerkmale als Hilfsmerkmale verwendet. Die Zuchtwerte können mittels dieser Methode auch früher abgeschätzt werden, da die Exterieurmerkmale bei den Töchtern eines Bullen in der ersten Laktation genauso früh erhoben werden können wie die Milchleistungsmerkmale. Die Ergebnisse der Studie von Bünger (1999) zeigen weiter im Detail, dass vor allem Eutermerkmale wie z. B. Euterboden, als ideale indirekte Hilfsmerkmale dazu dienen, die Nutzungsdauer zu schätzen und diese auch züchterisch zu bearbeiten. Somit lässt sich über diesen Zusammenhang der Trend im RZN erklären, vor allem aber auch die Zusammensetzung der Zuchtwertpunkte in den jüngeren Jahrgängen, da die Sicherheit der direkten Zuchtwerte für die Nutzungsdauer erst mit einer zunehmenden Anzahl abgegangener Kühe ansteigt. Die in den eigenen Untersuchungen festgestellte leichte Verbesserung des RZS kann, wie auch bereits der RZN, unter anderem auf den RZE zurückgeführt werden. Das belegt auch die Studie von Bünger (1999), die über die ermittelten mittleren bis hohen positiven genetischen Korrelationen des Merkmals Euterboden zu anderen Eutermerkmalen sowie zur Eutergesundheit auf diesen Zusammenhang schließt. Auch Swalve (1999) weist in seiner Untersuchung darauf hin, dass züchterische Verbesserungen des Exterieurs und der Nutzungsdauer auch positive Veränderungen in der Eutergesundheit hervorrufen. Weiterhin ist der RZS auch direkt im RZG gewichtet.

Eine weitere Verbesserung der untersuchten funktionalen Merkmale bei den Ökokühen wäre zukünftig wünschenswert, da, wie bereits in Kapitel 2.4 von Postler (2002a) beschrieben, eine hohe durchschnittliche Nutzungsdauer und eine gute Tiergesundheit maßgeblich die Remontierungs-, Tierarzt-, Futter- und Gebäudekosten bestimmen und somit auch die gesamte Wirtschaftlichkeit eines Betriebes beeinflussen. 


\subsubsection{Phänotypische Trends der Leistungsmerkmale bei Ökokühen}

Die phänotypischen Trends der Milchmenge der drei Laktationsklassen sind mitunter physiologisch bedingt. Folgende Argumente von Postler (2006) für die Zucht auf eine hohe Nutzungsdauer und hohe Lebensleistung dienen auch zur Interpretation der vorliegenden Ergebnisse. Demnach lassen sich die Steigerungen in der Milchmenge von Laktation zu Laktation dadurch erklären, dass der Organismus bis etwa zur vierten Laktation reifen muss, um ein Leistungsmaximum zu erreichen. Außerdem sind zu diesem Zeitpunkt die Organe soweit angepasst, dass eine hohe Grundfutterleistung durch ein höheres Futteraufnahmevermögen erreicht werden kann. Für Nauta et al. (2006a) sind die geringeren Kraftfuttergaben und die niedrige Rauhfutterqualität in Hinblick auf Energie und Protein Gründe für eine niedrige Milchleistung erstlaktierender Ökokühe im Vergleich zu konventionellen Kühen in der ersten Laktation. Die unterschiedlichen Niveaus der Fett- und Eiweißmengen und -gehalte sind vermutlich auch physiologisch bedingt und bei Erstlaktierenden entsprechend niedriger, da die Kühe in diesem Alter noch im Wachstum sind und somit mehr Nährstoffe benötigen als ältere Tiere.

Nauta et al. (2006a) schließen aufgrund der festgestellten Niveauunterschiede bei Fettund Eiweißgehalt wieder auf das Futter. Ihren Ergebnissen nach wird der niedrige Fettgehalt bei erstlaktierenden Ökokühen im Vergleich zu konventionellen Kühen durch die höheren Rauhfuttergaben bestimmt. Da diese von Kühen in höheren Laktationen aufgrund der nach Postler (2006) beschriebenen besseren Futterverwertung in eine höhere Grundfutterleistung umgesetzt werden können, liegt auch die Vermutung nah, dass mit zunehmender Laktation auch der Fettgehalt in der Milch ansteigt. Diese Vermutung könnte zumindest auch zur Erklärung der unterschiedlichen Niveaus für den Eiweißgehalt herangezogen werden. Die Ergebnisse von Nauta et al. (2006a) deuten auf einen negativen Trend im Merkmal Eiweißgehalt erstlaktierender Ökokühe hin. Den Autoren folgend liegt der Grund auch dafür im energieärmeren Futter und dabei speziell im eingeschränkten Angebot eiweißreicher Kraftfuttergaben.

\subsubsection{Phänotypische Trends in den funktionalen Merkmalen bei Ökokühen}

Die phänotypischen Leistungen der funktionalen Merkmale Zellzahl und Zwischenkalbezeit sind bei Betrachtung der einzelnen Laktationsgruppen sehr unterschiedlich. Von allgemein hohen Zellzahlniveaus auf ökologischen Betrieben, wie sie in der eigenen Studie in allen drei Laktationsklassen gefunden wurden, ist auch in den Studien von Nauta et al. (2006a) und Rozzi et al. (2007) die Rede. Nach den Argumenten von Nauta et al. (2006a) sind die Einschränkungen beim Einsatz von Antibiotika und die verbreitete Haltung der 
Kühe in Tiefstreuställen Gründe dafür, dass auf ökologischen Betrieben vermehrt Euterprobleme bzw. hohe Zellzahlen zu finden sind. Auch Rozzi et al. (2007) zeigen in ihrer Studie, dass ökologische Betriebe mit dem Verzicht auf den Einsatz von Antibiotika und anderen Medikamenten die meisten Schwierigkeiten mit Mastitis haben. Ebenso betonen beide Studien auch den auffällig kontinuierlichen Anstieg der Zellgehalte nach Umstellung der Betriebe von der konventionellen auf die ökologische Wirtschaftsform. Eine Erklärung der unterschiedlichen Niveaus der Einsatzleistungen im Merkmal Zellzahl könnten die von Fürst (2005) beschriebenen genetischen Korrelationen zwischen der Zellzahl und den Milchleistungsmerkmalen sein, da diese nach Angaben des Autors meist in dem Bereich zwischen 0,15 und 0,30 liegen. Weiter vermuten Nauta et al. (2006a), dass ältere, zu einem höheren Zellgehalt tendierende Kühe den Infektionsdruck in der Umwelt erhöhen und dadurch erstlaktierende Kühe anfälliger werden. In Übereinstimmung mit dieser Vermutung können die eigenen Ergebnisse interpretiert werden, in denen die Kühe in höheren Laktationen ebenfalls deutlich höhere Zellzahlen pro $\mathrm{ml}$ Milch aufweisen als Erstlaktierende.

Die Reduzierung der untersuchten Zwischenkalbezeit in den jüngeren Geburtsjahrgängen ökologischer Kühe deutet darauf hin, dass sich im Laufe der Jahre auch die Fruchtbarkeit auf den Ökobetrieben wesentlich verbessert hat. Bei einem direkten Vergleich der Zwischenkalbezeit auf ökologischen und konventionellen Betrieben stellten sowohl Reksen et al. (1999) als auch Nauta et al. (2006a) fest, dass es geringe Unterschiede bezüglich der Zwischenkalbezeit in beiden Betriebsformen gibt. Nauta et al. (2006a) ergänzen jedoch, dass der festgestellte Unterschied nicht signifikant ist und daher in Frage gestellt werden muss, ob die unterschiedliche Betriebsform als entscheidender Faktor bezüglich dieses Merkmals betrachtet werden kann. Reksen et al. (1999) kommen ebenfalls zu dem Schluss, dass die Fruchtbarkeitsleistungen ökologisch gehaltener Kühe mit selbigen konventioneller Kühe verglichen werden können. Mögliche längere Zwischenkalbezeiten bei ökologischen Kühen wären den Autoren zufolge auf eine schlechtere Fruchtbarkeit der Kühe bedingt durch ein niedriges Energieniveau im Futter zurückzuführen.

Eine Verringerung des Erstkalbealters bei den untersuchten Ökokühen würde sich vorteilhaft für die Wirtschaftlichkeit eines Betriebes auswirken. Neben Einsparung bei den Kosten für die Jungviehaufzucht kann der Stallplatz wieder schneller für ein neues Jungtier genutzt werden. Das für den letzten Geburtsjahrgang festgestellte Erstkalbealter von 27 Monaten ist vergleichbar mit dem Ergebnis aus der Studie von Nauta et al. (2006a). Hier zeigten Kühe nach der Umstellung im gesamten betrachteten Zeitraum einen Anstieg des Erstkalbealters von 1,75 Monaten. Im Vergleich dazu blieb das Erstkalbealter bei konven- 
tionellen Kühen durchgehend bei 26 Monaten. Anders als bei der eigenen Untersuchung weisen Nauta et al. (2006a) auf einen weiteren Anstieg des Erstkalbealters hin. Die Betriebsleiter besamen die Rinder erst zu einem späteren Zeitpunkt damit die Kalbung erst in einem höheren Alter und dementsprechend mit einem höheren Körpergewicht erfolgt. Ziel dieser späteren Erstkalbung soll sein, dass die Kühe sich besser an die spezifischen Anforderungen der ökologischen Milchviehhaltung anpassen können. Schlussfolgernd kann festgehalten werden, dass sich bei beiden Bullengruppen zufrieden stellende genetische Trends in den Merkmalen RZM, RZZ und RZE gezeigt haben. Um den Zuchtfortschritt in den Merkmalen RZN und RZS zu erhöhen, besteht für diese Merkmale Verbesserungsbedarf.

\section{Gesamtbewertung der Trends}

Insgesamt lassen die Ergebnisse darauf schließen, dass man durchaus mit einigen konventionellen Bullen auch unter ökologischen Bedingungen eine erfolgreiche Zucht realisieren kann. Die Niveauunterschiede zwischen Öko- und konventionellen Bullen könnten dadurch beeinflusst worden sein, dass pro untersuchtem Geburtsjahrgang unterschiedlich große Datensätze vorhanden waren. Für weitere statistische Analysen der ökologischen Milchviehhaltung und Vergleiche zu konventionellen Trends müsste eine größere Zahl ökologischer Betriebe identifiziert und der Datensatz weiter aufgestockt werden. Neben der vom VIT bemängelten Unvollständigkeit einiger Betriebsangaben können die untersuchten Trends auch dadurch beeinflusst worden sein, dass in der eigenen Untersuchung die Betriebe nicht wie bei Nauta et al. (2006a) nach dem Ökostatus bzw. in Umstellungsund langjährige Ökobetriebe eingeteilt worden sind. Um zukünftige Trends in der ökologischen Milchviehhaltung zu untersuchen, scheint die Differenzierung des Betriebsstatus sinnvoll, da auch Nauta et al (2006a) besonders im Bereich der Fütterung Unterschiede zwischen den neuen und den alten Ökobetrieben gefunden hat. Durch diese Klassifikation können schließlich die Trends sicherer interpretiert werden.

\subsection{ET-Status und genetisches Niveau der Ökobullen}

Bisherige Studien, die den ET-Status der Bullen auf ökologischen Betrieben untersucht haben, fehlen. Daher werden neben einem Fazit der vorliegenden Ergebnisse, kurz die möglichen Hintergründe zu den vorliegenden Kurvenverläufen erläutert.

Anhand der Ergebnisse der Kuhväter zeigte sich, dass etwa $42 \%$ der Bullen, die auf ökologischen Betrieben als Kuhväter identifiziert werden konnten, aus ET stammen. Allein bei der Rasse Holstein, die mit ca. 96 \% den größten Anteil an allen untersuchten Rassen 
darstellt, sind sogar etwa 43 \% der Väter ET-Bullen. Dieses Ergebnis ist nicht verwunderlich, da die herausgefilterten Schwarzbunten ET-Bullen hauptsächlich als Kuhväter in der konventionellen Haltung eingetragen sind und nach Angaben des BÖLW (2006) bei den Schwarzbunten Holstein-Friesian bereits ca. 80 \% aller Bullen aus Embryotransfer stammen. Bei der Betrachtung des Status des Kuhvaters fällt auf, dass die meisten Väter geprüfte KB-Bullen sind. Demgegenüber ist der Einsatz von Testbullen mit ca. $10 \%$ deutlich geringer als in konventionellen Zuchtbetrieben. Der scheinbare Anstieg der Deckbullen im letzten betrachteten Geburtsjahr der Kuh ist darauf zurückzuführen, dass diese Väter noch nicht komplett in der Zuchtwertdatei sind. Außerdem ist die Anzahl der Kühe im Geburtsjahrgang 2003 gering, da womöglich beim Datenschnitt noch nicht alle Kühe abgekalbt haben. Zusätzlich kann dieser Anstieg auch durch eine Verzerrung hervorgerufen worden sein, die durch den Einsatz von Bullen mit Töchtern, die ein durchschnittlich jüngeres Erstkalbealter besitzen, ausgelöst wird.

Der Trend bei der Auswahl von Anpaarungsbullen zeigt mit etwa $50 \%$, dass auch ökologische Betriebe nicht auf ET-Bullen bzw. geprüfte KB-Bullen verzichten. Auch Roeckl et al. (2005) beschreiben, dass nach aktuellem Stand der Zucht auf ökologischen Milchviehbetrieben, trotz der Richtlinienforderung den Natursprung zu bevorzugen, zu ca. $80 \%$ künstliche Besamung eingesetzt wird. Weil vermutlich für das Belegungsjahr 2005 noch nicht alle Kühe erfasst wurden, ist dieser Datensatz unvollständig und dementsprechend nicht so aussagekräftig.

Da wie bereits erwähnt über 80 \% ET-Bullen Schwarzbunte HF Bullen sind, sich das genetische Niveau zwischen ET- und Nicht-ET-Kuhvätern nicht unterscheidet und der genetische Trend Schwarzbunter Bullen bereits in Kapitel 5.1 dargestellt wurde, können die genetischen Trends der Kuhväter in gleicher Weise interpretiert werden.

\section{Gesamtbewertung der Untersuchung zum ET-Status}

Trotz der Probleme eines ungleichen Datenmaterials pro untersuchtem Geburts- bzw. Belegungsjahr beim Vergleich der ET- und Nicht-ET-Bullen kann dennoch gefolgert werden, dass die untersuchten Betriebe die ökologischen Rahmenbedingungen nur unzureichend erfüllen, indem sie ihre Kühe künstlich besamen und dem Anschein nach nicht berücksichtigen, ob der Bulle ein ET-Bulle ist oder nicht. Diese Reaktion impliziert, dass eine Beschränkung auf Nicht-ET Bullen für den Einsatz in der ökologischen Milchviehzucht, was aktuell stark in der Diskussion steht (vgl. hierzu z. B. Bapst und Spengler, 2006), die Auswahl bestimmter Bullen mit bestimmtem Profil deutlich einschränken würde. 


\subsection{Diskussion der in ZPLAN variierten Parameter}

Im folgenden Kapitel werden die Ergebnisse der Zuchtplanungsrechnungen einzelner Varianten eines ökologischen Zuchtprogramms diskutiert. Der Vergleich erfolgt zum größten Teil auf Basis konventioneller Studien, da bisherige Untersuchungen zur Ausgestaltung von ökologischen Zuchtprogrammen bzw. Erhebungen von Genotyp-UmweltInteraktionen zwischen ökologischen und konventionellen Betrieben kaum vorhanden sind (Swalve, 2006). Dazu werden die einzelnen Parameter, die den Aufbau eines Zuchtprogramms beeinflussen, nachfolgend dargestellt.

\subsubsection{Veränderung der Anzahl Testbullen und des Testanteils}

Wird bei der Untersuchung des Testanteils eine realistische Grenze von maximal $50 \%$ betrachtet, so kann das Ergebnis mit dem von Harder et al. (2002) verglichen werden, die für eine geschlossene ökologische Zuchtpopulation ebenfalls bei 30 Testbullen und einem Testanteil von $50 \%$ den höchsten monetären Zuchtfortschritt kalkulierten. Bei weniger als 30 Testbullen wird die Wahrscheinlichkeit geringer, ausreichend Topvererber zu erhalten. Da ein Testbulle, bis er einen Zuchtwert erhält, hohe Kosten verursacht (Gierzinger, 2002), erklärt dieser organisatorische Mehraufwand, der auch von Kalm und Harder (2003) beschrieben wird, die ansteigenden Züchtungskosten bei Erhöhung der Anzahl an Testbullen in diesem Zuchtprogramm und den dadurch bedingten Rückgang des Züchtungsgewinns.

Steigende Testkapazitäten bei gleich bleibender Anzahl Testbullen zeigen in den Ergebnissen einen kontinuierlichen Anstieg im erzielbaren monetären Zuchtfortschritt pro Jahr, aber auch im Züchtungsertrag und im Züchtungsgewinn. Hintergründe dafür liegen in einem kürzeren Generationsintervall, das sich aufgrund des verstärkten Einsatzes von Jungbullen ergibt. Außerdem wird, wie bereits erwähnt, durch einen höheren Testumfang die Erstellung größerer Töchterzahlen pro Testbulle realisiert, durch die schließlich eine genauere Zuchtwertschätzung der geprüften Altbullen gewährleistet wird (Kalm und Harder, 2003).

Aufgrund der Tatsache, dass die Zuchtverbände ihre Einnahmen vor allem aus dem Verkauf von Sperma geprüfter Bullen beziehen und auch Landwirte hohen Testanteilen skeptisch gegenübertreten, gelten Testanteile über $50 \%$ als eher unrealistisch. Daher werden höhere Testanteile in der eigenen Arbeit nicht weiter untersucht. Zudem zeigt sich eine Beschränkung der Anzahl an Testbullen monetär als vorteilhaft. Somit kann abschließend 
festgehalten werden, dass sich für dieses Basiszuchtprogramm mit 30 Testbullen und einem Testanteil von $50 \%$ die beste monetäre Variante ergibt.

\subsubsection{Verschiebung der Gewichtung der Fitnessmerkmale}

Anders als in den Studien von Baumung und Sölkner (1999), Baumung et al. (2001), Kalm und Harder (2003) sowie Gredler (2004), wurden in der eigenen Untersuchung im Zuge der Variation der Subindices „Fitness“, die Produktionsmerkmale Fett-kg und Eiweiß-kg nicht konstant gehalten sondern um den gleichen Anteil in die entgegen gesetzte Richtung verändert. Im Ergebnis wird deutlich, dass sich trotz der Verringerung bzw. Erhöhung der Gewichtung der Produktionsmerkmale ein vergleichbares Resultat einstellt wie bei den angeführten Studien. Durch die Erhöhung der wirtschaftlichen Gewichte der Fitnessmerkmale nimmt die relative ökonomische Bedeutung zugunsten dieses Merkmalkomplexes zu. Darauf begründet sich der deutlich größere Anteil der Fitnessmerkmale am monetären Zuchtfortschritt. Bei einer Erhöhung der ökonomischen Gewichte der Fitnessmerkmale um $50 \%$ zeigen Berechnungen, dass die ökonomische Effizienz bei über $90 \%$ liegt (Baumung und Sölkner, 1999; Baumung et al., 2001; Kalm und Harder, 2003). Somit stellt die Selektion auf Fitnessmerkmale und die Vernachlässigung der Produktionsmerkmale kein großes wirtschaftliches Risiko dar. Diese Folgerung ist vor allem für die ökologische Milchviehhaltung von Bedeutung, da der Fokus des ökologischen Zuchtziels auf den Fitnessmerkmalen liegt (vgl. hierzu Postler und Schmidt, 2003; Postler, 2006) und auch Baumung und Sölkner (1999) erwähnen in ihrer Studie, dass eine direkte Erhöhung der wirtschaftlichen Gewichte der funktionalen Merkmale als einfache Möglichkeit dienen kann, ein stärker ökologisch orientiertes Zuchtziel zu verwirklichen. Die Umsetzung wird jedoch maßgeblich durch subjektive Einstellungen aufgrund des hohen Stellenwertes der Milchmerkmale in der Praxis beeinträchtigt (Baumung et al., 2001).

In Folge der stärkeren Gewichtung der Produktionsmerkmale nehmen alle Erfolgsparameter nach Verringerung der wirtschaftlichen Gewichte der Fitnessmerkmale um $50 \%$ deutlich zu. Der hohe Anteil des Merkmalkomplexes Milch am gesamten monetären Zuchtfortschritt deutet darauf hin, dass auch dann kein hohes wirtschaftliches Risiko besteht, wenn nur nach Milch selektiert und die Fitness vernachlässigt wird. Im Merkmalskomplex Fitness ist entsprechend ein Rückgang der naturalen Zuchtfortschritte und somit auch eine Verschlechterung des Anteils am gesamten monetären Zuchtfortschritt zu erkennen. Obwohl sich durch diese Maßnahme der Merkmalskomplex Milch deutlich verbessert, ist eine solche Verschlechterung der Fitnessmerkmale unter ökologischen Gesichtspunkten nicht erstrebenswert. Die Veränderungen der gesamten Untersuchung sind schließlich 
auch auf die Robustheit des Selektionsindexes (Philipsson et al., 1994) zurückzuführen. Demnach bleibt der Selektionsindex auch durch die kleinste Veränderung der ökonomischen Gewichte unbeeinflusst. Es kommt lediglich zur Verschiebung der Anteile der Merkmalsgruppen innerhalb dieses stabilen Selektionsindexes, was auch in den eigenen Ergebnissen vorzufinden ist.

\subsubsection{Einfluss unterschiedlicher Populationsgrößen}

Die positiven Entwicklungen in allen untersuchten Erfolgsparametern bei Erhöhung der Populationsgröße zeigen, dass die Größe der Population einen positiven Einfluss auf den Erfolg eines Zuchtprogramms hat. Durch den Anstieg der Populationsgröße verbessert sich die Selektionsintensität auf dem Bullenvaterpfad. Zu diesem Ergebnis kommen auch Kalm und Harder (2003), die diesen Einfluss als Hauptgrund für den Anstieg des Zuchtfortschritts und des Züchtungsgewinns aufführen. Ebenso besitzen größere Populationen auch größere Testkapazitäten, wodurch mehr Jungbullen mit einer ausreichenden Anzahl an Töchtern für die Nachkommenprüfung auf Milchleistungsmerkmale geprüft werden können. Bei den eigenen Ergebnissen muss allerdings beachtet werden, dass es sich bei den modellierten Zuchtprogrammen nicht um geschlossene Ökopopulationen handelt. Somit kann an dieser Stelle, wie es auch Kalm und Harder (2003) als typisch für ein konventionelles Zuchtprogramm beschreiben, der Einsatz von Embryotransfer bei den Bullenmüttern nicht ausgeschlossen werden. In diesem Fall lassen sich die steigenden Erfolgsparameter größerer Populationen, auch durch die Steigerung der Reproduktionsrate weiblicher Tiere, durch eine höhere Selektionsintensität im Bullenmütter- und Kuhmütterpfad und durch Nutzung von Vollgeschwisterinformationen für die Zuchtwertschätzung erklären.

Durch eine Verringerung der Populationsgröße innerhalb der eigenen Modellkalkulationen, verschlechtern sich auch die Erfolgsparameter, da die Selektion der Bullenväter und die Testkapazität eingeschränkt werden. Folglich veranschaulicht dieses Ergebnis, dass sich ein eigenes ökologisches Zuchtprogramm langfristig nur mit einer ausreichend großen Population realisieren lassen würde (vgl. hierzu Kalm und Harder, 2003).

\subsubsection{Variation der Töchterleistung pro Anzahl Testbesamungen}

Bei der Variation der Töchterleistung pro Anzahl Besamungen zeigte sich, dass mit besseren Verhältnissen kleiner als 1:10 mehr monetärer Zuchtfortschritt und ein höherer Züchtungsgewinn erreicht wird. Vergleichbare Ergebnisse zeigt die Studie von Gierzinger 
(2002). Durch eine hohe Wiederfindungsrate können mehr Töchterinformationen gewonnen werden, die den Informationsgehalt und somit die Genauigkeit der Zuchtwertschätzung steigern. Außerdem kann bei einem guten Verhältnis der gewonnenen Töchterleistungen pro Besamung die Spermaproduktion eingeschränkt werden, was zu leichten Einsparungen in den Züchtungskosten und zum Anstieg im dargestellten Züchtungsgewinn führt.

Der kontinuierliche Rückgang bei zunehmend schlechteren Verhältnissen lässt sich maßgeblich durch den mangelnden Informationsgehalt für die Zuchtwertschätzung erklären. Eine mögliche Ursache für die abnehmende Wiederfindungsrate ist der Export von Rindern, die von Testbullen abstammen. Aber auch Rinder die aufgrund mangelnder Fruchtbarkeit nicht tragend werden und der Einsatz von Testbullen auf Betrieben, die nicht im Herdbuch sind, wirken sich negativ auf den Informationsgehalt und den hier dargestellten Züchtungsgewinn aus. Ferner führt Gierzinger (2002) auch eine ungleichmäßige Verteilung der Testbullen durch die Besamer und eine sehr lange Ausgabedauer als Faktoren an, die den Informationsgehalt einschränken. Für ein ökologisches Zuchtprogramm sollte somit ebenfalls ein enges Verhältnis bzw. hohe Wiederfindungsraten zwischen der Töchterleistung und der Anzahl Besamungen angestrebt werden.

Die einzige Schwachstelle in der Untersuchung der Töchterleistung pro Anzahl Besamungen stellt nach Gierzinger (2002) die fehlende Berücksichtigung eventueller Totalausfälle von Testbullen, die durch nicht erreichte Mindestsicherheiten der geschätzten Zuchtwerte auftreten können, dar.

\subsubsection{Einsatz künstlicher Besamung}

Der seitens der Rahmenbedingungen des ökologischen Landbaus gewünschte Verzicht auf den Einsatz künstlicher Besamung erweist sich für dieses Zuchtprogramm als nicht sinnvoll. Zu dem gleichen Schluss kommen auch Kalm und Harder (2003), die sich ebenfalls mit der Frage der Notwendigkeit eines eigenen ökologischen Zuchtprogramms befassen. Die Ergebnisse zeigen, dass ein totaler Verzicht auf künstliche Besamung zu einem starken Verlust im Züchtungsgewinn führt, da die Kosten höher liegen als der Ertrag. Dieser Effekt ist u. a. darauf zurückzuführen, dass sich die Selektionsintensität bei Natursprungbullen verringert, da aus einer bestimmten Anzahl potentieller Bullen eine immer größere Zahl an Tieren selektiert wird. Ferner führen Kalm und Harder (2003) mit an, dass sich mit Rückgang der künstlichen Besamung die aktive Zuchtpopulation verkleinert, wodurch die Selektionsintensität im Bullenmütterpfad reduziert wird und sich die Anzahl 
Testbullen pro Jahr verringert. Die Erhöhung des Anteils künstlicher Besamungen in der Zuchtstufe lässt den Züchtungsgewinn wiederum ansteigen. Ein Grund dafür ist neben den bereits erwähnten Einflussfaktoren eine schnellere Realisierung des Zuchtfortschritts durch den Einsatz von künstlicher Besamung (vgl. hierzu Rahmann, 2004).

Der höchste Züchtungsgewinn, der in der vorliegenden Studie bei einem Anteil von 80 \% künstlicher Besamung in der Zuchtstufe und $80 \%$ in der Produktionsstufe erreicht wird, ist vergleichbar mit dem vorherrschenden von Roeckl et al. (2005) auf ökologischen Praxisbetrieben erhobenen Anteil künstlicher Besamung von ca. 80 \%. Um monetäre Verluste und eine Reduzierung der aktiven Zuchtpopulation zu vermeiden, sollte in Anlehnung an die Ergebnisse der Anteil künstlicher Besamung nicht unter $50 \%$ fallen. Da auch für Natursprungbullen keine Nachkommenprüfung stattfindet und die Genauigkeit der Zuchtwertschätzung mit steigender Anzahl Natursprungbullen immer geringer wird, wäre ein eigenes ökologisches Zuchtprogramm ohne künstliche Besamung in diesem Fall nicht rentabel. Aus diesen Gründen empfehlen Kalm und Harder (2003), den Anteil künstlicher Besamung in einem ökologisches Zuchtprogramm auf 50 \% zu setzen, um die Kosten zur Durchführung rechtfertigen zu können. Auch Rahmann (2004) erwähnt, dass v. a. bei kleineren Kuhherden die künstliche Besamung kostengünstiger ist als ein eigener Bulle.

\subsubsection{Verteilung der Kühe auf die Zucht- und Produktionsstufe}

Aufgrund der stärkeren Selektionsintensität in der Zuchtstufe, wird mehr Selektionserfolg erreicht als vergleichsweise in der Produktionsstufe. Insofern kann mit einem steigenden Anteil an Kühen in der Zuchtstufe mehr Zuchtfortschritt und mehr Züchtungsertrag realisiert werden. Bei einem Anteil von $60 \%$ Kühen in der Zuchtstufe und $40 \%$ in der Produktionsstufe ist der Züchtungsgewinn am größten, da vermutlich bei diesem Verhältnis der Grenzertrag den Grenzkosten entspricht. Der Rückgang des Züchtungsgewinns mit steigender Anzahl an Kühen in der Zuchtstufe, wäre demnach auf die durch das Gesetz des abnehmenden Ertragszuwachses bedingte Verringerung des Grenzertrags zurückzuführen (Kräusslich, 1994). 


\subsubsection{Alter der Kühe bei Geburt der ersten Nachkommen}

Bei der Untersuchung des Alters der Kühe bei der Geburt der ersten Nachkommen zeigt sich, dass der meiste Züchtungsgewinn dann erreicht wird, wenn die Kühe möglichst jung abkalben. Der Züchtungsertrag und der Züchtungsgewinn sind hier bei einem Alter von 24 Monaten am höchsten. Hintergründe dafür sind aus ökonomischer Sicht die niedrigeren Produktionskosten bei der Rinderaufzucht, die sich durch geringeren Arbeitsaufwand und Einsparungen in den Futter- und Gebäudekosten ergeben. Neben den geringen Aufzuchtkosten pro Einzeltier, führt ein frühes Erstkalbealter auch zu Einsparungen der Remontierungskosten der gesamten Herde. Weiterhin kann bei einem frühen Erstkalbealter auch mehr Zuchtvieh verkauft werden, was sich neben der Nutzung des genetischen Fortschritts, aufgrund einer besseren Selektion sowie einem kürzeren Generationsintervall, ebenfalls positiv auf den Züchtungsgewinn auswirkt (Sutter, 2006).

Ein zu junges Alter bei Geburt der ersten Nachkommen ist trotz hohen ökonomischen Nutzens aus ökologischer Sicht nicht wünschenswert. Um den ökologischen Zielen entgegenzukommen, kann aus den vorliegenden Ergebnissen ein Erstkalbealter von mindestens 2,2 Jahren, besser 2,4 Jahren, bis maximal 2,6 Jahre empfohlen werden. In diesem Altersbereich zeigt sich die Abnahme des Züchtungsgewinns nicht so stark wie bei einem höheren Alter. Trotz abnehmender Züchtungsgewinne ist dieses Alter aus ökologischer Sicht als positiv zu bewerten, da nicht nur Experten, sondern auch Züchter ein höheres Erstkalbealter in diesem Bereich favorisieren. Demgemäß soll gewährleistet werden, dass sich die Kühe bis zur Geburt der ersten Nachkommen besser an die spezifischen Anforderungen der ökologischen Milchviehhaltung anpassen können und ihr Organismus entsprechend heranreifen kann (vgl. hierzu Postler, 2002a; Nauta et al., 2006a). Sutter (2006) belegt schließlich mit den Ergebnissen seiner Studie, dass sich ein höheres Körpergewicht vorteilhaft auf die 305-Tage-Leistung auswirkt.

\subsubsection{Auswirkungen der Genotyp-Umwelt-Interaktionen}

In allen untersuchten Merkmalen wurde der Züchtungsgewinn durch Herabsetzen der Heritabilität reduziert. Die Zunahme des konventionellen Einflusses und der dadurch ausgelösten Verringerung der Ökopopulation zeigten Reduktionen in den Züchtungskosten und ließen somit den Züchtungsgewinn in den offenen Zuchtprogrammen ansteigen. Dennoch ist der Züchtungsertrag im geschlossenen Zuchtprogramm am höchsten, da bei einem offenen ökologischen Zuchtprogramm nur eine geringere Anzahl an Ökokühen für Testbesamungen zur Verfügung steht, die Anzahl der Nachkommen sinkt und dadurch nur geringer Züchtungsertrag realisiert werden kann. Bedingt durch den geringen Wert 
des Genanteils konventioneller Bullen, zeigen kleiner werdende genetische Korrelationen innerhalb der offenen Zuchtprogramme auch geringere Züchtungserträge und weniger Züchtungsgewinn. Die Umrangierung der Bullen in Bezug auf die naturalen Zuchtfortschritte, erfolgte aufgrund des durch kleiner werdende genetische Korrelationen bedingten Anstiegs konventioneller Bullen, da sich ihre Zuchtwerte nicht auf die Bullen der Ökopopulation übertragen lassen.

Die auch bei Weigel et al. (1999), Kearney et al. (2004) und Gerber et al. (2006) festgestellte Umrangierung der Bullen unter extensiven Bedingungen, wurde ebenfalls durch die Einschränkung des Leistungspotentials der konventionellen Bullen hervorgerufen. Jedoch waren die Differenzen, wie auch die eigene Untersuchung zeigt, nicht groß genug, um im Bereich der Zuchtwertschätzung eine Differenzierung nach Umwelten vorzunehmen. Aber auch die den Studien von Weigel et al. (1999), Kearney et al. (2004), Fürst (2006), Gerber et al. (2006) und Nauta et al. (2006b) und den projektinternen Ergebnissen (Bapst und Stricker, 2006; Reinhardt, 2006) zu entnehmenden hohen genetischen Korrelationen für Leistungsmerkmale, sind Gründe dafür, dass noch keine Notwendigkeit zur Gestaltung eines eigenen geschlossenen Ökozuchtprogramms besteht. Überlegungen kämen nach Nauta et al. (2006b) erst dann in Frage, wenn die genetische Korrelation der Milchleistung unter 0,8 fallen würde. Schwarzenbacher (2002), Fürst (2006) und Gerber et al. (2006) weisen aber auch darauf hin, dass diese hohen Korrelationen vermutlich aufgrund der relativ geringen Leistungsunterschiede zwischen konventionellen und ökologischen Betrieben zu Stande kommen.

Die von Reinhardt (2006) ermittelten Genotyp-Umwelt-Interaktionen bei funktionalen Merkmalen lassen sich vermutlich auf den im ökologischen Landbau verbotenen Einsatz von Antibiotika und anderen Medikamenten zurückführen. Die dadurch verursachten höheren Zellgehalte auf ökologischen Milchviehbetrieben führen möglicherweise zu vermehrten Abgängen und dementsprechend zu einer Verkürzung der Nutzungsdauer. Da aber die Studie von Fürst (2006) gegenteiliges, also genetische Korrelationen im Merkmal Zellzahl nahe 1 belegen, sind weitere Analysen im Bereich der Fitnessmerkmale notwendig, um detaillierte Aussagen bezüglich Wechselwirkungen zwischen den Betriebstypen treffen zu können. 
Gesamtbewertung der Auswirkungen zu Genotyp-Umwelt-Interaktionen

Im Hinblick auf die eigene Untersuchung würde unter den gegebenen Voraussetzungen und Parametern ein geschlossenes ökologisches Zuchtprogramm keinen höheren Zuchtfortschritt, wohl aber deutlich höhere Züchtungskosten verursachen als ein offenes Zuchtprogramm mit Genfluss aus dem konventionellen in den ökologischen Bereich. Die Entwicklung eines eigenen Zuchtprogramms für die ökologische Milchviehhaltung würde sich unter diesem Gesichtspunkt nur bedingt lohnen. Außerdem zeigen alle hier dargestellten Ergebnisse, besonders die der Leistungsmerkmale, dass sich unter konventionellen Bedingungen geprüfte Besamungsbullen in gleicher Weise für den Einsatz auf ökologischen Milchviehbetrieben eignen, um auch dort entsprechende Zuchtfortschritte zu erzielen. Trotz dieser Tatsache behalten Genotyp-Umwelt-Interaktionen auch zukünftig einen hohen Stellenwert in der Zucht. Vor allem trifft dies auf die ökologische Milchviehhaltung zu, da Nauta et al. (2006b) vermuten, dass die genetischen Korrelationen aufgrund der erhöhten Rauhfuttergaben zukünftig geringer werden. 


\section{Schlussfolgerung}

Die Untersuchungen zur Erhebung der genetischen Trends für Leistungsmerkmale und funktionale Merkmale zeigen, dass in der ökologischen Milchviehzucht häufig die gleichen Bullen eingesetzt werden, die auch in der konventionellen Milchviehzucht zum Einsatz kommen. Dadurch zeichnet sich keineswegs ein Nachteil ab, da aus den Ergebnissen hervorgeht, dass man durchaus mit ausgewählten konventionellen Bullen auch unter ökologischen Bedingungen eine erfolgreiche Zucht realisieren kann. Ob sich ein ähnliches Ergebnis bei einer den ökologischen Rahmenbedingungen entsprechenden Beschränkung der Auswahl auf Nicht-ET Bullen zeigen würde, bleibt zu bezweifeln, da die Auswahl bestimmter Bullen mit einem bestimmten Profil und damit die Zuchtentscheidungen der Betriebe deutlich einschränkt werden würden. Schließlich wäre ein deutlicher Niveauunterschied in den einzelnen Trends zu erwarten.

Bei den Kühen ist anhand der positiven Trends für das Merkmal Milchmenge deutlich zu erkennen, dass die Genetik der Tiere verbessert worden ist, was zu einer Leistungssteigerung geführt hat. Obwohl eine weitere Verbesserung in den funktionalen Merkmalen wünschenswert ist, lässt sich erkennen, dass auf den Betrieben das stärker auf Fitnessmerkmale ausgerichtete ökologische Zuchtziel bereits in der Praxis umgesetzt wird. Um zukünftig Trends in der ökologischen Milchviehzucht zu untersuchen, scheint eine Klassifizierung der Betriebe nach dem Umstellungsjahr sinnvoll, da somit auch die Genetik der Tiere und mögliche Umwelteinflüsse wie z. B. Fütterungsunterschiede besser beurteilt werden können und die Trendentwicklung genauer interpretiert werden kann.

Aus den Zuchtplanungsrechnungen wird ersichtlich, dass sich langfristig nur mit einer den konventionellen Bedingungen entsprechenden Populationsgröße und dem Einsatz von künstlicher Besamung ein Züchtungserfolg realisieren lässt. Der Einsatz künstlicher Besamung würde jedoch bei der Umsetzung eines eigenen ökologischen Zuchtprogramms gegen die Regeln der EU-Verordnung zum ökologischen Landbau verstoßen. Ein weiteres wünschenswertes Kriterium, um den Grundsätzen einer ökologischen Milchviehzucht zu entsprechen, ist die Verbesserung der funktionalen Merkmale. Neben der untersuchten Erhöhung der wirtschaftlichen Gewichte in diesem Merkmalskomplex würde auch ein verstärkter Einsatz an Testbullen zum gewünschten Zuchtfortschritt führen. Durch eine Erhöhung der Testkapazität bei gleicher Anzahl getesteter Bullen können trotz geringer Heritabilitäten der funktionalen Merkmale die naturalen Zuchtfortschritte innerhalb dieses Merkmalkomplexes deutlich verbessert werden. 
Bezüglich der Rentabilität eines geschlossenen ökologischen Zuchtprogramms zeigt die Untersuchung zu Genotyp-Umwelt-Interaktionen zwischen konventionellen und ökologischen Milchviehbetrieben, dass im Vergleich zu einem offenen Zuchtprogramm kein höherer Zuchtfortschritt, aber wesentlich höhere Züchtungskosten entstehen würden und sich somit eine Differenzierung als nicht lohnend erweist. Insgesamt kann also festgehalten werden, dass mit dem Einsatz von unter konventionellen Bedingungen geprüften Besamungsbullen und einer aktiveren Beteiligung der ökologischen Betriebe an konventionellen Zuchtprogrammen entsprechend hohe Zuchtfortschritte im Bereich der ökologischen Milchviehzucht realisiert werden können. 


\section{Zusammenfassung}

Beeinflusst durch die vermehrte Nachfrage des Verbrauchers nach Produkten aus dem ökologischen Landbau und aufgrund staatlicher Förderprogramme für tiergerechte Haltungssysteme hat in den vergangenen Jahren die Zahl der ökologisch wirtschaftenden Betriebe in Deutschland zugenommen. Insbesondere der Bereich der ökologischen Milchproduktion wächst kontinuierlich. Dabei unterliegt diese im Vergleich zur konventionellen Produktionsweise wesentlich strengeren Anforderungen an die Zucht und Haltung der Kühe. Eine Anpassung der züchterischen Konzepte an die spezifischen Bedingungen ist zu diesem Zweck notwendig, um sowohl die Konkurrenzfähigkeit und Effizienz der ökologischen Milchproduktion zu steigern als auch den gesamten Sektor nachhaltig zu etablieren. Das vorrangige Ziel dieser Arbeit liegt infolgedessen darin, möglichst realistische und praxisrelevante Szenarien zu entwickeln und unter den gegebenen Rahmenbedingungen und Zuchtzieldefinitionen Voraussetzungen für eine effektive Ausgestaltung von Zuchtprogrammen im Bereich der nachhaltigen Zucht ökologischer Milchrinder zu schaffen.

Anhand der vom VIT zur Verfügung gestellten Daten von 442 ökologisch wirtschaftenden Milchviehbetrieben konnten genetische und phänotypische Trends für Leistungs- und funktionale Merkmale erhoben werden. Die deutlichsten Trends zeigen sich für Milchleistung $(+78,16 \mathrm{~kg}$ pro Jahr) und Exterieur, während für die Merkmale Nutzungsdauer, Fruchtbarkeit und Eutergesundheit kaum ein Zuchtfortschritt zu verzeichnen ist. Einen allgemein positiven Trend zeigen die Relativzuchtwerte der Kühe. Die Trends der Kuhväter entwickeln sich mit Ausnahme des Fettgehaltes, des maternalen Kalbeverlaufes, der paternalen Totgeburtenrate und des RZS ebenfalls positiv. Eine weitere Analyse zum ETStatus der Bullen legt dar, dass aktuell $43 \%$ der auf ökologischen Milchviehbetrieben eingesetzten Kuhväter der Rasse Holstein-Friesian ET-Bullen sind. Die meisten Väter sind geprüfte KB-Bullen und der Einsatz von Testbullen ist mit ca. $10 \%$ deutlich geringer als in konventionellen Betrieben.

In einem weiteren Schritt erfolgten Evaluierungen verschiedener Zuchtplanungsalternativen mit Hilfe des Computerprogramms ZPLAN. Die darin über die genetischen Korrelationen für die Merkmale Eiweißmenge, Zellzahl und Nutzungsdauer variierten Genotyp-Umwelt-Interaktionen zeigen, dass ein geschlossenes ökologisches Zuchtprogramm keinen höheren Zuchtfortschritt, aber deutlich höhere Züchtungskosten als ein offenes Zuchtprogramm verursachen würde. 
Ist der Einsatz von künstlicher Besamung im vorliegenden Basiszuchtprogramm mit einer Populationsgröße von 109.600 Kühen zulässig, empfiehlt sich der Einsatz von 30 Testbullen mit einer Testkapazität von 50 \%. Mit dieser Kombination wird ein optimaler Züchtungsgewinn von $22,11 €$ pro Kuh bezogen auf die Investitionsperiode erreicht. Die Erhöhung der wirtschaftlichen Gewichte für funktionale Merkmale um $50 \%$ stellt kein großes wirtschaftliches Risiko dar, was in Bezug auf das ökologische Zuchtziel mit Fokus auf den Fitnessmerkmalen von großer Bedeutung ist. Ebenfalls risikoarm, aber aus ökologischer Sicht nicht erstrebenswert, ist die Selektion auf Milchleistung bei gleichzeitiger Vernachlässigung der Fitnessmerkmale.

Der laut EU-Verordnung gewünschte Verzicht auf den Einsatz künstlicher Besamung erweist sich für das angenommene Zuchtprogramm als nicht sinnvoll. Um monetäre Verluste und eine Reduzierung der aktiven Zuchtpopulation zu vermeiden, sollte der Anteil an künstlicher Besamung in der Zuchtstufe nicht unter 50 \% fallen. Bei der Wahl des Erstkalbealters sollte trotz hohen ökonomischen Nutzens auf ein zu junges Alter verzichtet werden, um den Kühen zu ermöglichen, sich besser an die spezifischen Anforderungen der ökologischen Milchviehhaltung anzupassen und ihren Organismus heranreifen zu lassen.

Abschließend ist festzuhalten, dass sich ökologische Milchviehbetriebe in ihrem praktizierten züchterischen Verhalten von den konventionell wirtschaftenden Betrieben nur wenig unterscheiden. Unter diesen Umständen ist die Gestaltung eines eigenen ökologischen Zuchtprogramms als wenig effizient und wirtschaftlich nicht rentabel einzustufen. Wünschenswert wäre aber eine aktivere Beteiligung der ökologisch wirtschaftenden Betriebe an den etablierten Zuchtprogrammen. 


\section{Literaturverzeichnis}

Abdallah , J.M. und McDaniel, B.T. (2000): Genetic Parameters and Trends of Milk, Fat, Days Open, and Body Weight After Calving in North Carolina Experimental Herds. Journal of Dairy Science 83: 1364-1370.

Anacker, G. (2003): Hochleistung und Tiergesundheit bei Milchkühen. Archiv für Tierzucht, Dummerstorf 46 (2003), Sonderheft: 57-62.

Bapst, B. und Spengler, A. (2006): Nicht nur auf Höchstleistung setzen. Tierzucht und Tierhaltung, Tätigkeitsbericht 2006, FiBL-Forschungsinstitut für biologischen Landbau Frick (CH): 26.

Bapst, B. und Stricker, C. (2006): Schätzung der Genotyp x Umwelt Interaktion zwischen ökologisch und konventionell wirtschaftenden Betrieben anhand Schweizer Produktionsdaten. Projektinterne Präsentation zum 4. Projekttreffen ÖKUH, 8. Dezember 2006, Göttingen, (bisher unveröffentlicht).

Barth, K. und Rahmann, G. (2005): Milcherzeugung im ökologischen Landbau. In: Brade, Wilfried und Flachowsky, Gerhard, (Hrsg.). Rinderzucht und Milcherzeugung Empfehlungen für die Praxis, 2. Auflage, Kapitel 7, 136-144. Sonderhefte der Landbauforschung Völkenrode 289. Bundesforschungsanstalt für Landwirtschaft $(\mathrm{FAL})$.

Baumung, R. (2006): Allgemeine Grundlagen der Zucht. 4. Fachtagung für Schafhaltung, Irdning/Österreich, 17. November 2006.

Baumung, R. und Sölkner, J. (1999): Ökologischer Gesamtzuchtwert - was müsste anders sein? In: Zuchtziele beim Rind, Seminar des genetischen Ausschusses der Zentralen Arbeitsgemeinschaft österreichischer Rinderzüchter, Salzburg, 18. März 1999: 38-46.

Baumung, R., Sölkner, J., Gierzinger, E., Willam, A. (2001): Ecological total merit index for an Austrian dual purpose cattle breed. Archiv für Tierzucht, Dummerstorf 44: 5-13.

Bergfeld, U. (2004): Zukünftige Anforderungen an die Rinderzucht - Konsequenzen für die Leistungsprüfung- und Zuchtwertschätzung. Tierzuchttagung der Deutschen Gesellschaft für Züchtungskunde, Humboldt-Universität zu Berlin, 11.06.2004: 6378.

Bijma, P., Meuwissen, T.H.E., Wolliams, J.A. (2002): Design of sustainable breeding programs in developmed countries. Proceedings of the $7^{\text {th }}$ World Congress on genetics Applied to Livestock Production, August 19-23. 2002, Montpellier, France, 2401.

BMELV (2007): Ökologischer Landbau in Deutschland. Stand: Januar 2007. Bundes-ministerium für Ernährung, Landwirtschaft und Verbraucherschutz, http://www.bmelv.de/cln_045/nn_750590/DE/04Landwirtschaft/OekologischerLand bau/OekologischerLand $\bar{b}$ auDeutschland.html_nnn=true, 13.04.2007

Bo, N. (2004): Das skandinavische System der Erfassung von Erkrankungen und dessen züchterische Nutzung. Herausforderungen für die Gestaltung der Rinderzucht von morgen, Internationaler Rindertag, Leipzig, 04. März 2004, In: DGfZ-Schriftenreihe, Heft 34: 115-124. 
Boelling, D., Groen, A.F., Sørensen, P., Madsen, P., Jensen, J. (2003): Genetic improvement of livestock for organic farming systems. Livestock Production Science 80: 79-88.

BÖLW (2006): Braucht der Öko-Landbau eine eigene Tierzucht? Eine nachhaltige Viehwirtschaft erfordert vielfältige Zuchtziele. In: Nachgefragt: 25 Antworten zum Stand des Wissens rund um Öko-Landbau und Bio-Lebensmittel. Bund Ökologische Lebensmittelwirtschaft e.V. (Hrsg.): 20-21.

Brade, W. (2005): Nutzungsdauer und Abgangsursachen von Holsteinkühen: Konsequenzen für die Züchtung? Praktischer Tierarzt 86, Ausgabe 9: 658-667.

Bünger, A. (1999): Die Länge des produktiven Lebens und ihre Beziehung zu linearen Exterieurmerkmalen bei Holstein-Friesian Kühen. Dissertation, Georg-AugustUniversität Göttingen.

Chagunda, M.G., Bruns, E.W., King, J.M., Wollny, C.B.A. (1999): Genotype by environment interaction for milk yield of Canadian Holstein Friesian on large scale dairy farms in Malawi. Deutscher Tropentag in Berlin, Session: Sustainable Technology Development in Animal Agriculture.

Costa, C.N., Blake, R.W., Pollack, E.J., Oltenacu, P.A., Quaas, R.L., Searle, S.R. (2000): Genetic analysis of Holstein cattle populations in Brazil and the United States. Journal of Dairy Science 83: 2963-2974.

Cromie, A.R., Kelleher, D.L., Gordon, F.J., Rath, M. (1997): Genotype by environment interaction for milk yield in dairy cattle. Irish Grassland and Animal Production Association Journal 31: 29-35.

DESTATIS (2005): Viehbestand in landwirtschaftlichen Betrieben mit ökologischem Landbau. Statistisches Bundesamt Deutschland, http://www.destatis.de/print/php, 30.03.2006.

DHV (2007): Zuchtziel Deutsche Holsteins. Deutscher Holstein Verband e.V. - Holsteinzucht, http://www.holstein-dhv.de/deutsch/holstzu/zuzi.htm, 03.04.2007.

Dosch, T. und Gerber, A. (2007): Die Ökologische Lebensmittelwirtschaft in Deutschland 2006: Zahlen, Daten, Fakten. Bilanzpressekonferenz des BÖLW, Biofach 14.02.2007.

Duklee, J.S., Freemann, A.E., Kelley, D.H. (1994): Comparison of Holsteins Selected for High and Average Milk Production. 1. Net Income and Production Response to Selection for Milk. Journal of Dairy Science 77: 1890-1896.

Ertl, M. und A. Haiger (2005): Naturgemässe Milchrinderzucht - Zuchtziel zwischen ökonomischen Wünschen und ökologischen Grenzen. AÖLZ Stierkatalog 2005.

Essl, A. (1999): Grundsatzfragen zum Zuchtziel beim Rind. In: Zuchtziele beim Rind, Seminar des genetischen Ausschusses der ZAR, Salzburg, 18. März 1999: 3-9.

Falconer, D.S. (1984): Einführung in die quantitative Genetik. Eugen Ulmer GmbH \& Co, Stuttgart. 
FAO (2001): Guidelines for the Production, Processing, Labelling and Marketing of Organically Produced Foods. Food and Agriculture Organization of the United Nations, GL 32-1999, Rev. 1-2001, Rome, Italy.

Fewson, D. (1993): Definition of the breeding objective. Design of livestock breeding programs, Animal Genetics and Breeding Unit, The University of New England, Armidale NSW, Australien.

FiBL (2001): Der ökologische Gesamtzuchtwert ÖZW - Ein grosser Schritt in Richtung Biokuh. FiBL-Forschungsinstitut für biologischen Landbau, CH-Frick, Merkblatt 2001.

Fürst, C. (2005): Züchterische Strategien hinsichtlich zukünftiger Anforderungen an Milch und Milchinhaltsstoffe. 32. Viehwirtschaftliche Fachtagung, Irdning/Österreich, 13.14. April 2005.

Fürst, C. (2006): Züchterische Strategien für die Bio-Rinderzucht. Österreichische Fachtagung für biologische Landwirtschaft, Irdning/Österreich, 21.-22. März 2006: 3746.

Gierzinger, E. (2002): Zuchtplanung und Optimierung der Zuchtprogramme für die österreichische Fleckviehpopulation. Dissertation, Universität für Bodenkultur Wien.

Grandke, R. (2002): Methoden und Modelle zur strategischen Steuerung von neuzeitlichen Zuchtorganisationen. Habilitationsschrift, Justus-Liebig-Universität zu Giessen.

Gredler, B. (2004): Einfluss wirtschaftlicher Gewichte und genetischer Parameter auf den Zuchtfortschritt der Zuchtprogramme Fleckvieh Austria und Braunvieh Austria. Diplomarbeit, Universität für Bodenkultur, Wien.

Grothe, P.O. (1993): Holstein-Friesian, eine Rasse geht um die Welt. Landwirtschaftsverlag $\mathrm{GmbH}$, Münster-Hiltrup.

Haiger, A. (2006): Milchviehzucht - Vorgangsweise der Arbeitsgemeinschaft österreichischer Lebensleistungszüchter (AÖLZ). Österreichische Fachtagung für biologische Landwirtschaft, Irdning/Österreich, 21.-22. März 2006.

Harder, B., W. Junge, J. Bennewitz und E. Kalm (2002): Zuchtplanung für die Rinderzucht im ökologischen Landbau. Vortragstagung der DGfZ und GfT am 18./19. September in Halle.

Heckenberger, G.J. (1991): Planungsrechnungen über den Einfluß von Grenznutzenwerten der Leistungsmerkmale, Parametern der Populationsstruktur und von Züchtungssystemen auf den Züchtungserfolg beim Zweinutzungsrind. Dissertation, Universität Hohenheim.

Hill, W.G. (1974): Prediction and evaluation of response to selection with overlapping generations. Animal Production 18: 117-139.

Hollenberg-Koch, H. und Schoch, R. (2004): Bio-Milchpreise folgen konventionellem Markt. Öko Markt Spezial, Informationen der ZMP Zentrale Markt- und Preisberichtstelle für Erzeugnisse der Land-, Forst- und Ernährungswirtschaft $\mathrm{GmbH}$, Sonderausgabe zur EuroTier 2004. 
Kalm, E. und B. Harder (2003): Ökologische Tierzucht: Status Quo, Möglichkeiten und Erfordernisse in der Rinder- und Schweinezucht. Abschlussbericht, Universität Kiel.

Kearney, J.F., Schutz, M.M., Boettcher, P.J., Weigel, K.A. (2004): Genotype x Environment Interaction for Grazing Versus Confinement. I. Production Traits. Journal of Dairy Science 87: 501-509.

König, S. (2001): Untersuchungen zu einem kooperativen Zuchtprogramm der Rasse Holstein-Friesian. Dissertation, Georg-August-Universität Göttingen.

König, S., Dietl, G., Raeder, I., Swalve, H.H. (2005): Genetic Relationships for Dairy Performance Between Large-Scale and Small-Scale Farm Conditions. Journal of Dairy Science 88, pp. 4087-4096.

König, S. und Swalve, H.H. (2000): Nutzung der Ergebnisse der InterbullZuchtwertschätzung zum Vergleich des genetischen Niveaus von Ländern. Vortragstagung der DGfZ und GfT am 20./21. September 2000 in Kiel.

Kräusslich, H. (1994): Tierzüchtungslehre. 4. Auflage, Eugen Ulmer GmbH \& Co, Stuttgart.

Krogmeier, D., Anzenberger, H., Rosenberger, E., Wimmer, A. (2007): Der Ökologische Gesamtzuchtwert für Fleckvieh, Braunvieh und Gelbvieh Februar 2007. LfLInformation, Bayerische Landesanstalt für Landwirtschaft, FreisingWeihenstephan.

Lind, B. (2006): Ökonomische Gewichte für Merkmale des Gesamtzuchtwertes. 4. Rinderworkshop, Uelzen 2006:139-147.

Mc Clintock, A. E. und Cunningham, E.P. (1974): Selection in dual purpose cattle populations: Defining the breeding objective. Animal Production 18: 237-247.

MUNLV.NRW (2002): Ministerium für Umwelt und Naturschutz, Landwirtschaft und Verbraucherschutz des Landes Nordrhein-Westfalen. Fachinformationssystem Ökologischer Landbau. http://nrw.oekolandbau.de/tierhaltung/1101.phtml, 15.09.2004.

Nauta, W.J., Baars, T., Groen, A.F., Veerkamp, R.F., Roep, D. (2001): Animal breeding in organic farming. Discussion paper, Louis Bolk Instituut, Driebergen.

Nauta, W.J., Baars, T., Bovenhuis, H. (2006a): Converting to organic dairy farming: Consequences for production, somatic cell scores and calving interval of first parity Holstein cows. Livestock Science 99: 185-195.

Nauta, W.J., Veerkamp, R.F., Brascamp, E.W., Bovenhuis, H. (2006b): Genotype by Environment Interaction for Milk Production Traits Between Organic and Conventional Dairy Cattle Production in The Netherlands. Journal of Dairy Science 89: 27292737.

Nieberg, H. und Deeken, E. (2005): Wie stehen die deutschen Biomilcherzeuger im internationalen Wettbewerb? Tagungsbeitrag der 1. Internationalen BiolandMilchviehtagung, Loccum, 16.-17.2.2005. 
Nicholas, F.W. und Smith, C. (1983): Increased rates of genetic change in dairy cattle by embryo transfer and splitting. Animal Production 36: 341-353.

Nitter, G., Bartenschlager, H., Karras, K., Niebel, E., Graser, H.-U. (2000): ZPLAN: a PC computer program to optimize livestock selection schemes. User's Guide for ZPLAN, Version 2000, University Hohenheim.

NOG (2007): NOG Gesellschafter. Nord-Ost-Genetik, Verden / Aller, http://nog.de/start.htm, 28. März 2007.

Peters, K.J. (1993): Selection and breeding strategies for sustainable livestock production. In: Developing countries, with particular reference to dairy cattle production, editors: M. Gill, E. Owen, G.E. Pollot and T.L.J. Lawrence, British Society of Animal Production Occasional Publication No.16: 119-128.

Philppson, J., Banos, G., Arnason, T. (1994): Present and Future Uses of Selection Index Methodology in Dairy Cattle. Journal of Dairy Science 77: 3252-3261.

Postler, G. (2002a): Wie erwirtschafte ich mit geringst möglichem Aufwand den höchsten Gewinn in der Milchviehhaltung, Kostenreduktion, Aufwand/Ertrags-Verhältnis. 29. Viehwirtschaftliche Fachtagung, Irdning/Österreich, 24.-25. April 2002.

Postler, G. (2002b): Naturgemäße Rinderzucht - Ganzheitliche Betrachtungsweise in der naturgemäßen Viehwirtschaft, Heft 1.

Postler, G. (2003): Vom Auerochs zur Turbokuh - „Lebenszeit“ in der Tierzucht. Kirche im ländlichen Raum, 02/2003: 18-22.

Postler, G. (2005): LL-Bullenkatalog für Rinderzucht auf Lebensleistung 2006.

Postler, G. (2006): Ökologischer Gesamtzuchtwert (ÖZW) in der Milchviehhaltung. Österreichische Fachtagung für biologische Landwirtschaft. Irdning/Österreich, 21.-22. März 2006: 47-49.

Postler, G. und Schmidt, G. (2003): Pionierprojekte für ökologische Rinderzucht. Ökologie \& Landbau 128, 4/2003.

Pryce, J.E., Nielsen, B.L., Veerkamp, R.F., Simm, G. (1999a): Genotype and feeding system effects and interactions for health and fertility traits in dairy cattle. Livestock Production Science 57: 193-201.

Pryce, J.; Simm, G., Veerkamp, R.F., Oldham, J.D. (1999b): Genotype by nutrition interactions from Langhill dairy cows in three lactations. Proceedings of the British Society of Animal Science, 200.

Rahmann, G. (2004): Ökologische Tierhaltung. Eugen Ulmer GmbH \& Co.

Redelberger, H. et al. (2002): Ökologische Milchviehhaltung. Fachinformation des ÖkoTeams Hessen, HDLGN Hessisches Dienstleistungszentrum für Landwirtschaft, Gartenbau und Naturschutz.

Reinhardt, F. (2006): Schätzung der Genotyp x Umwelt Interaktion zwischen ökologisch und konventionell wirtschaftenden Betrieben anhand ausgewählter Modellbetriebe in Deutschland. Projektinterne Präsentation zum 4. Projekttreffen ÖKUH, 8. Dezember 2006, Göttingen, (bisher unveröffentlicht). 
Reksen, O., Tverdal, A., Ropstad, E. (1999): A Comparative Study of Reproductive Performance in Organic and Conventional Dairy Husbandry. Journal of Dairy Science 82: $2605-2610$.

Rendel, J.M. und Robertson, A. (1950): Estimation of genetic gain in milk yield by selection in a. closed herd of dairy cattle. Journal of Genetics 50: 1-8.

Rippin, M. (2006): Milch. In: Ökomarkt Jahrbuch 2006 - Verkaufspreise im ökologischen Landbau 2004/2005, Materialien zur Marktberichterstattung, Band 60, ZMP Zentrale Markt- und Preisberichtstelle GmbH (Hrsg.), Bonn.

Roeckl, C., Rusche, B., Gottwald, F.-T. (2005): Gesunde Leistung - Der neue Tierzuchtfonds und die Suche nach Zuchtalternativen für die bäuerliche und ökologische Landwirtschaft. Der kritische Agrarbericht 2005: 126-130.

Ron, M. und Hillel, J. (1983): Genotype x environment interaction in dairy cattle and its role in breeding programmes. TAG - Theoretical and Applied Genetics 66, Springer Verlag, pp. 93-99.

Rozzi, P., Miglior, F., Hand, K.J. (2007): A Total Merit Selection Index for Ontario Organic Dairy Farmers. Journal of Dairy Science 90: 1584-1593.

Sato, K., Bartlett, P.C., Erskine, R.J., Kaneene, J.B. (2004): A comparison of production and management between Wisconsin organic and conventional dairy herds. Livestock Production Science 93: 105-115.

Schmidt, G. (2003): Auf dem Weg zu einer ökologischen Tierzucht. Ökologie \& Landbau 128, 4/2003: 6-10.

Schumacher, U. (2002): Milchviehfütterung im ökologischen Landbau. Bioland Verlags $\mathrm{GmbH}$.

Schumacher, U. (2004): Strategisches Hygienemanagement im ökologischen Milchviehbetrieb. Fachvortrag auf der Eurotier 2004, Hannover, 10.11.2004.

Schüler, L., Swalve, H.H., Götz, K.U. (2001): Grundlagen der Quantitativen Genetik. Verlag Eugen Ulmer, Stuttgart.

Schwarzenbacher (2001): Vergleich von biologischen mit konventionellen Milchviehbetrieben in Niederösterreich. Diplomarbeit, Institut für Nutztierwissenschaften der Universität für Bodenkultur, Wien.

Schwarzenbacher, H. (2002): Erfordern unterschiedliche Leistungsgrenzen auch unterschiedliche Zuchttiere?. Leistungszucht und Leistungsgrenzen beim Rind, Seminar des genetischen Ausschusses der ZAR, Salzburg, 2002: 36-41.

Skjervold, H. (1966): Die künstliche Besamung als bedeutendes Hilfsmittel moderner Rinderzucht. Schriftenreihe „Der Tierzüchter“, Heft 2.

SOEL (2006): Richtlinien und Gesetzgebungen zum ökologischen Landbau. Stiftung Ökologie \& Landbau, http://www.soel.de/oekolandbau/richtlinien.html, 22.08.2006. 
Stratmann, J. (2006): Genetische und phänotypische Trends für Leistungs- und Sekundärmerkmale in ökologisch wirtschaftenden Milchviehbetrieben. Bachelorarbeit im wissenschaftlichen Studiengang Agrarwissenschaften, Institut für Tierzucht und Haustiergenetik, Universität Göttingen, (unveröffentlicht).

Sutter, F. (2006): Optimales Erstkalbealter von Aufzuchtrindern aus ökonomischer und physiologischer Sicht. 33. Viehwirtschaftliche Fachtagung, Irdning/Österreich, 26.27. April 2006: 11-17.

Swalve, H. (1999): Gibt es Grenzen in der Zucht auf Milchleistung? - Aus der Sicht der Züchtung. Vortrag zur Jahrestagung der Deutschen Gesellschaft für Züchtungskunde e.V. am 14. September in Alsfeld, In: Züchtungskunde, 71: 428-436.

Swalve, H. (2006): Tierzucht im Ökologischen Landbau. Thüringer ÖkolandbauFachtagung, 24.01.2006.

Swalve, H. und Höver, K. (2003): Untersuchungen an den Ergebnissen der Zuchtwertschätzung von Holstein Bullen und Kühen in Deutschland. Archiv für Tierzucht, Dummerstorf 46: 113-126.

TopQ (2007): TopQ-Partner für Sie, TopQ, http://topq-partner.de/, 28. März 2007

van Tassel, C.P. und van Vleck, L.D. (1991): Estimates of genetic selection differentials and generation intervals for four paths of selection. Journal of Dairy Science 74: 1078-1086.

VIT (2006a): Neuigkeiten der Zuchtwertschätzung Mai 2006 - Jährliche Basisanpassung der Relativzuchtwerte. Vereinigte Informationssysteme Tierhaltung w. V. Verden I Aller.

VIT (2006b): Zuchtwertschätzung. Jahresbericht 2006 des VIT. Vereinigte Informationssysteme Tierhaltung w. V. Verden / Aller.

VIT (2007): Beschreibung der Zuchtwertschätzung für Milchleistungsmerkmale, Zellzahl, Exterieurmerkmale, Nutzungsdauer und Zuchtleistungsmerkmale, Stand: Februar 2007. Vereinigte Informationssysteme Tierhaltung w. V. Verden / Aller.

Vogt, G. (2001a): Geschichte des ökologischen Landbaus im deutschsprachigen Raum Teil I. Ökologie \& Landbau 118, 2/2001: 47-49.

Vogt, G. (2001b): Geschichte des ökologischen Landbaus im deutschsprachigen Raum Teil II. Ökologie \& Landbau 119, 3/2001: 47-49.

Walter, L. (2003): Milchviehzucht für ökologische Betriebe - Kritische Auseinandersetzung zur aktuellen Frage der Notwendigkeit einer eigenen Zucht für die ökologische Landwirtschaft. Diplomarbeit im Fachbereich Ökologische Agrarwissenschaften, Universität Kassel.

Weigel, K. A., Kriegl, T., Pohlman, A.L. (1999): Genetic Analysis of Dairy Cattle Production Traits in a Management Intensive Rotational Grazing Environment. Journal of Dairy Science 82: 191-195. 
Willam, A. (1997): Kuhmütter und Stiermütter: Bedeutung der Kuh für den Zuchtfortschritt. In: Die Stellung der Kuh im Zuchtgeschehen, Von Stiermüttern, Kuhmüttern und Kuhfamilien, Seminar des genetischen Ausschusses der ZAR, Salzburg, 13. März 1997: 3-11.

Willam, A. (2007): persönliche Mitteilung.

Zwald, N.R., Weigel, K.A., Fikse, W.F., Rekaya, R. (2003): Identification of Factors That Cause Genotype by Environment Interaction Between Herds of Holstein Cattle in Seventeen Countries. Journal of Dairy Science 86: 1009-1018. 


\section{Danksagung}

Die vorliegende Arbeit wurde am Institut für Tierzucht und Haustiergenetik der GeorgAugust-Universität angefertigt. Im Laufe meiner Promotion haben viele Personen auf ihre Weise zum Gelingen dieser Arbeit beigetragen, denen ich an dieser Stelle herzlich danken möchte.

Mein besonderer Dank gilt Prof. Dr. Henner Simianer für die Überlassung des Themas, die sehr lehrreiche Zusammenarbeit und die gewährleisteten Freiräume sowie die Möglichkeit und das Vertrauen, meine Ergebnisse auf Konferenzen und Tagungen im In- und Ausland vorzustellen. Der fachliche Austausch und das angenehme Arbeitsklima in seiner Abteilung werden auch zukünftig dafür sorgen, dass ich an die Promotionszeit in Göttingen gerne zurückdenke.

Ausdrücklich bedanken möchte ich mich auch bei Frau PD Dr. Roswitha Baumung von der BOKU Wien für die spontane Übernahme des Korreferats.

Auch bei Herrn Ass. Prof. Dr. Alfons Willam von der BOKU Wien möchte ich mich für die Bereitstellung des Computerprogramms ZPLAN, die Hilfestellung und wertvollen Ratschläge bei der Anwendung bedanken.

Ebenso bin ich dem VIT für die Bereitstellung der Betriebsdaten zu Dank verpflichtet.

Bei der Bundesanstalt für Landwirtschaft und Ernährung (BLE) und dem Bundesministerium für Ernährung, Landwirtschaft und Verbraucherschutz (BMELV) möchte ich mich für die Projektbetreuung und die finanzielle Unterstützung bedanken.

Die Kollegen am Institut haben das Ihrige für eine wunderbare Zeit in Göttingen beigetragen. Bei Herrn Dr. Sven König möchte ich mich für seine fachliche Unterstützung und die ständige Diskussionsbereitschaft bedanken. Tamina danke ich für die stets positive Arbeitsatmosphäre im Büro und für ihre Geduld mit mir. Bianca und Sven aus dem ,Rinderberatungsbüro' bin ich für ihre stetige Ansprechbarkeit und die Diskussionsbereitschaft in fachlichen und weniger fachlichen Angelegenheiten dankbar. Friederike danke ich für das schnelle Korrekturlesen meiner Arbeit und für ihre Aufmunterungen. Weiterhin bedanke ich mich bei Annabell, Holger, Rebecca, Maria, Daniel und Stephan, für die schöne und erlebnisreiche Zeit in Göttingen. 
Mein ganz besonderer Dank gilt Torsten, der jederzeit für mich da war, mir Mut gemacht und mich durchweg liebevoll unterstützt hat.

Schließlich gilt mein größter Dank meinen Eltern und Geschwistern Sebastian, Kerstin und Romy für ihre liebevolle Unterstützung und ihr großes Vertrauen. Danke, dass ihr mir stets bedingungslos zur Seite gestanden habt, immer für mich da seid und mir diese Ausbildung ermöglicht habt. 